\title{
INTERACTIONS BETWEEN WAVEMAKING AND THE BOUNDARY LAYER AND WAKE OF A SHIP MODEL
}

by

A. Shahshahan and L. Landweber

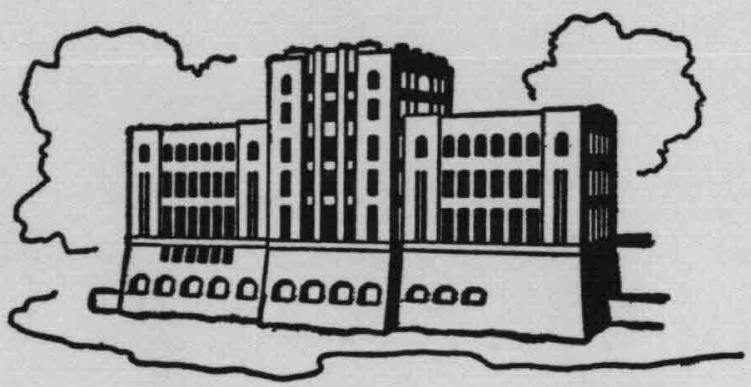

IIHR Report No. 302

Iowa Institute of Hydraulic Research

The University of Iowa

Iowa City, Iowa 52242

May 1986 


\section{INTERACTIONS BETWEEN WAVEMAKING AND THE BOUNDARY LAYER AND WAKE OF A SHIP MODEL}

by

A. Shahshahan and L. Landweber

IIHR Report No. 302

Iowa Institute of Hydraulic Research

The University of Iowa

Iowa City, Iowa 52242

May 1986 


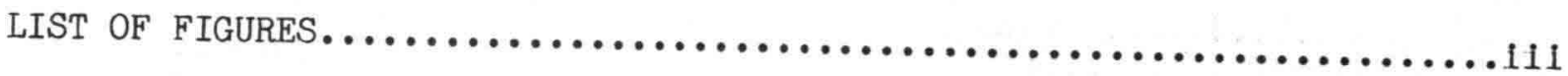

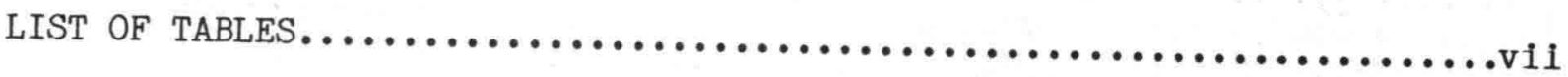

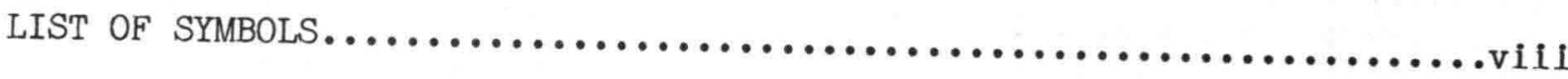

ABSTRACT.

ACKNOWLEDGEMENTS.

CHAPTER

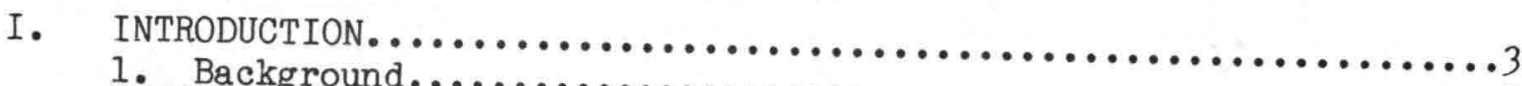

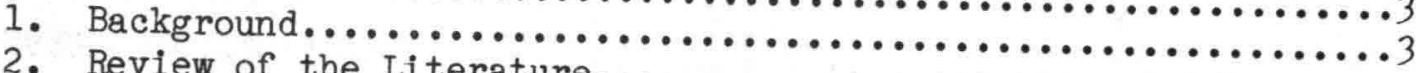

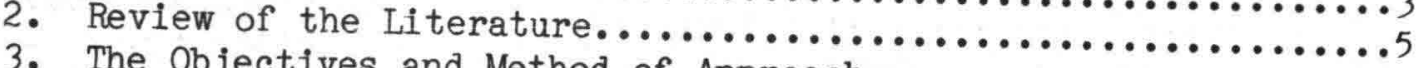

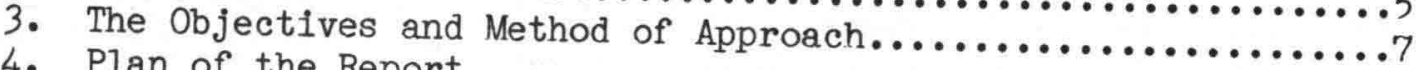

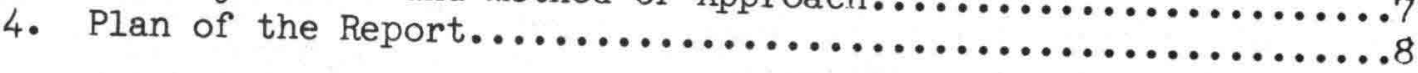

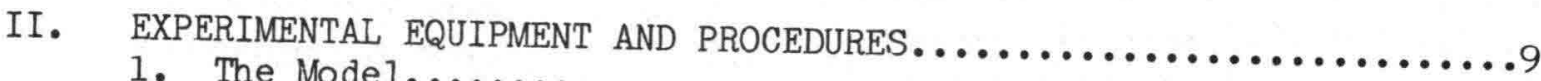

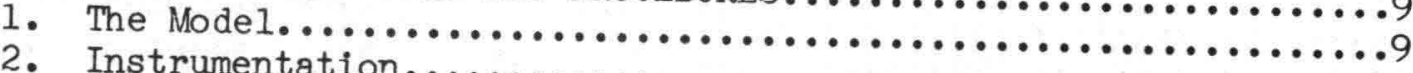

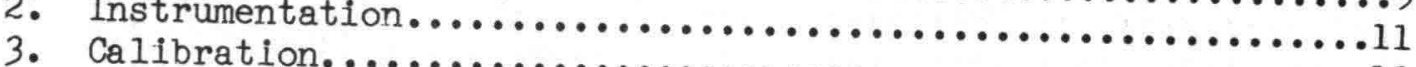

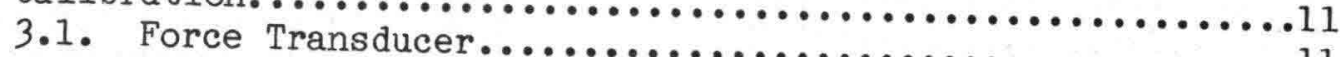

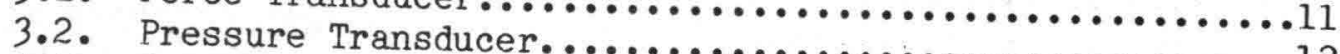

3.3. Calibration of $5-\mathrm{H}$.

4. Data-Acquisition System.

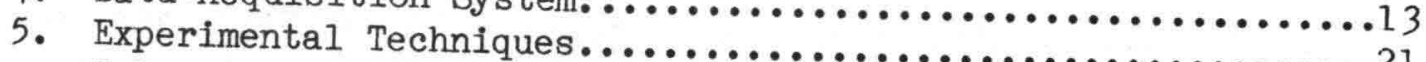

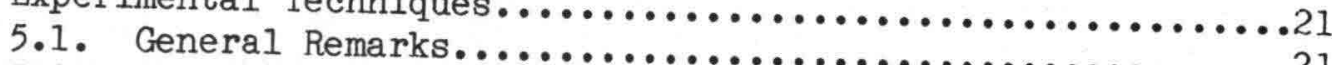

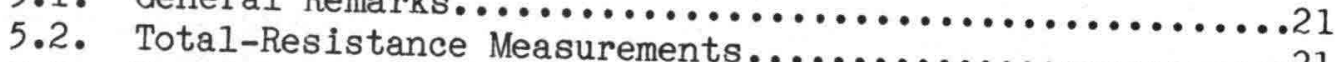

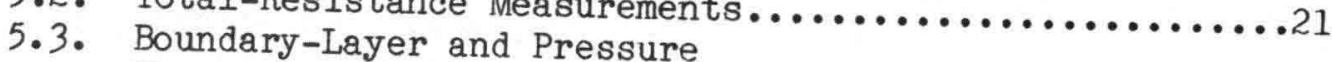

Measurement

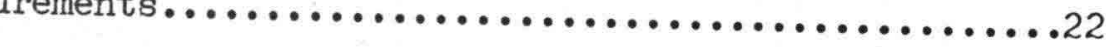

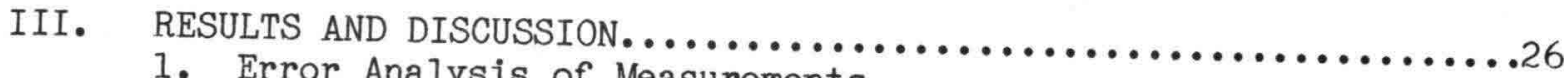

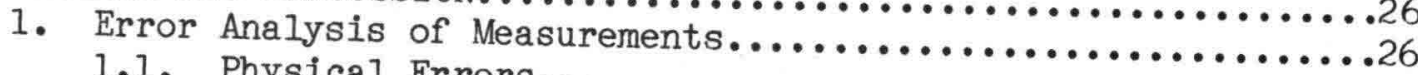

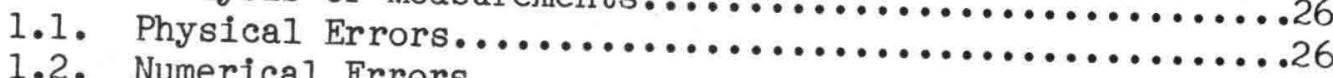

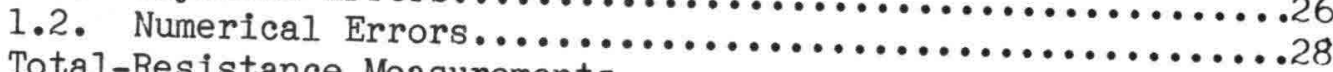

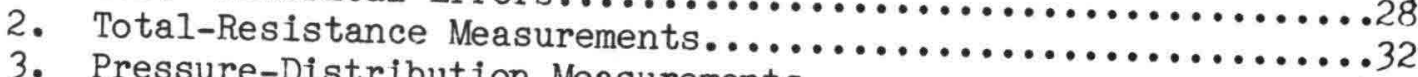

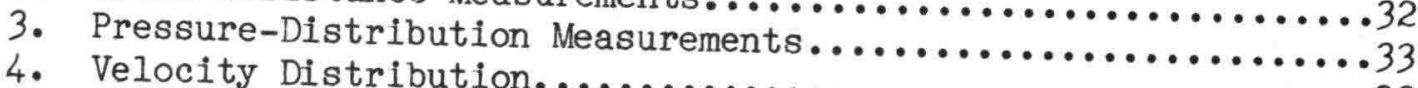

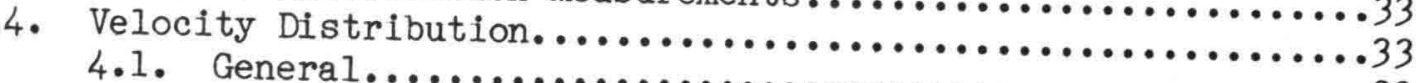

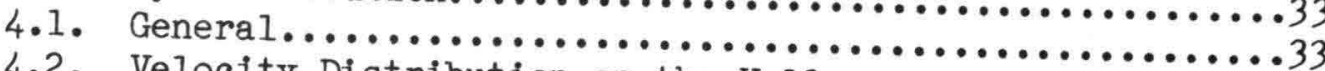

5. Wake Measurements......................

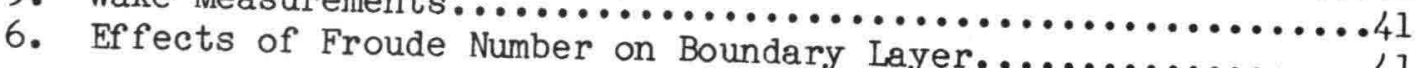

IV. BOUNDARY-LAYER CALCULATIONS FOR THE WIGLEY HULL............78

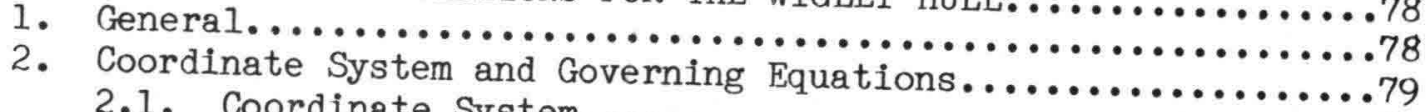

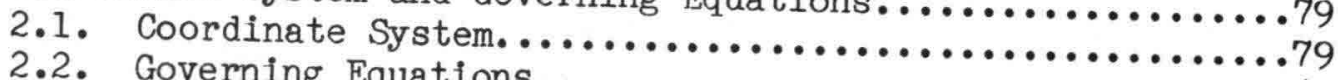

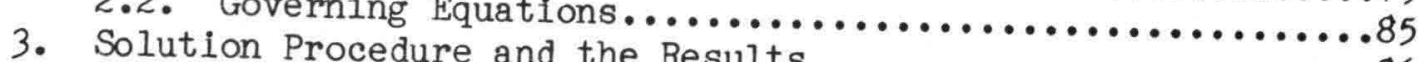


3.1. Solution Procedure...............................86

3.2. Calculation Results.................................... 87

V. WAVE-RESISTANCE CALCULATIONS..............................101

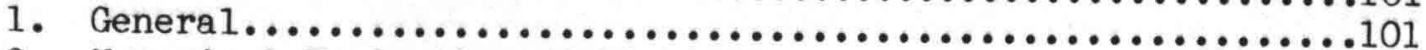

2. Numerical Evaluation of Michell's

Integral for the Wigley ShIp Form....................101

3. Wave Resistance without the Effects

of Boundary Layer...................................104

3.1. Thin-Ship Approximation............................104

3.2. Hull-Surface Distribution............................

3.3. Slender-Body Solution for the

Centerplane Distribution.......................106

4. Wave Resistance with the Ef fect of

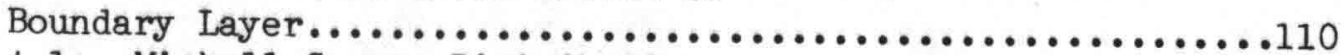

4.1. Michell Source Distribution

with Viscous Effects.........................112

4.2. Slender-Body Solution with

Viscous Effects.............................116

4.3. Hull-Surface Distribution with

Viscous Effects...............................116

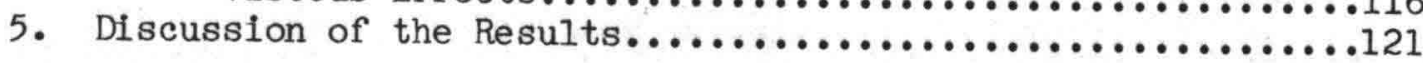

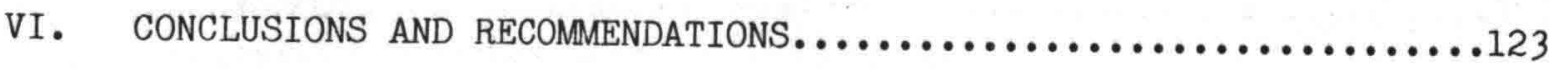

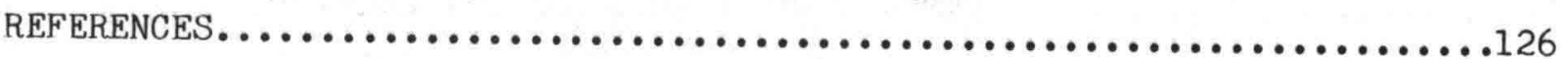




\section{LIST OF FIGURES}

Figure

Page

1. Wigley hull model......................................

2. Definition sketch for the yaw probe (pitch \& yaw)...............14

3. Yaw probe calibration curves for pitch angles..................15

4. Yaw probe calibration curves for yaw angles....................16

5. Yaw probe calibration curves for the magnitude of velocity $\ldots . . . . .17$

6. Yaw probe calibration curves for the local static pressure........28

7. Dynamic calibration curves for five-hole yaw probe...............19

8. Data-acquisition system for the carriage speed and boundary layer and wake measurements.

9. Body plan and measuring positions of Wigley model..............25

10. Comparison of $C_{t}$ 's vs. Froude number (Free condition)...........30

11. Comparison of $C_{t}{ }^{\prime} s$ and $C_{w}$ 's vs. Froude number (Fixed condition).

12. Comparison of measured and calculated pressure

distribution $(\mathrm{Fr}=0.266)$

13. Comparison of measured and calculated pressure

distribution $(\mathrm{Fr}=0.313)$

14. Comparison of measured and calculated pressure

distribution $(\mathrm{Fr}=0.350)$

15. Comparison of measured and calculated pressure

distribution $(\mathrm{Fr}=0.400)$

16. Measured streamwise velocity component $(|\mathrm{z} / \mathrm{H}|=0.2) \ldots \ldots \ldots \ldots \ldots . .44$

17. Measured streamwise velocity component $(|\mathrm{z} / \mathrm{H}|=0.4) \ldots \ldots \ldots \ldots \ldots$

18. Measured streamwise velocity component $(|\mathrm{z} / \mathrm{H}|=0.6) \ldots \ldots \ldots \ldots \ldots . . .46$

19. Measured streamwise velocity component $(|\mathrm{Z} / \mathrm{H}|=0.8) \ldots \ldots \ldots \ldots \ldots 7$

20. Measured streamwise velocity component $(|\mathrm{Z} / \mathrm{H}|=1.0) \ldots \ldots \ldots \ldots \ldots . . .48$ 
21. Vector plot of $\mathrm{w}-\mathrm{v}$ components $(2 \mathrm{x} / \mathrm{L}=0.5, \mathrm{Fr}=0.266) \ldots \ldots \ldots \ldots \ldots 49$

22. Vector plot of $\mathrm{w}-\mathrm{v}$ components $(2 \mathrm{x} / \mathrm{L}=0.5, \mathrm{Fr}=0.313) \ldots \ldots \ldots \ldots$

23. Vector plot of $\mathrm{w}-\mathrm{v}$ components $(2 \mathrm{x} / \mathrm{L}=0.5, \mathrm{Fr}=0.350) \ldots \ldots \ldots \ldots . . .51$

24. Vector plot of $\mathrm{w}-\mathrm{v}$ components $(2 \mathrm{x} / \mathrm{L}=0.5, \mathrm{Fr}=0.400) \ldots \ldots \ldots \ldots . .52$

25. Vector plot of $\mathrm{w}-\mathrm{v}$ components $(2 \mathrm{x} / \mathrm{L}=0.86, \mathrm{Fr}=0.266) \ldots \ldots \ldots \ldots . .53$

26. Vector plot of $\mathrm{w}-\mathrm{v}$ components $(2 \mathrm{x} / \mathrm{L}=0.86, \mathrm{Fr}=0.313) \ldots \ldots \ldots \ldots . .54$

27. Vector plot of $\mathrm{w}-\mathrm{v}$ components $(2 \mathrm{x} / \mathrm{L}=0.86, \mathrm{Fr}=0.350) \ldots \ldots \ldots \ldots 55$

28. Vector plot of $\mathrm{w}-\mathrm{v}$ components $(2 \mathrm{x} / \mathrm{L}=0.86, \mathrm{Fr}=0.400) \ldots \ldots \ldots \ldots . . .56$

29. Measured streamwise velocity component in the wake

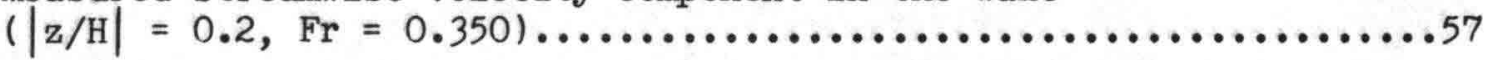

30. Measured streamwise velocity component in the wake

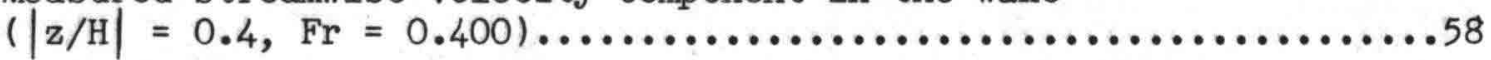

31. Measured streamwise velocity component in a cross

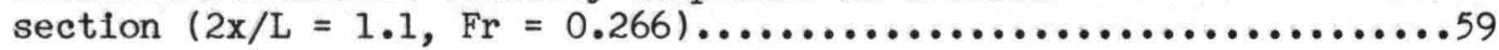

32. Measured streamwise velocity component in a cross

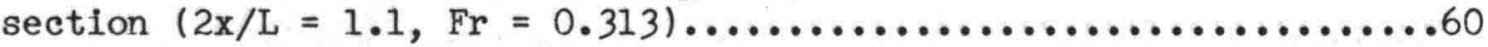

33. Vector plot of $\mathrm{w}-\mathrm{v}$ component $(2 \mathrm{x} / \mathrm{L}=1.0$, $\mathrm{Fr}=0.266)$.

34. Vector plot of $\mathrm{w}-\mathrm{v}$ component $(2 \mathrm{x} / \mathrm{L}=1.0, \mathrm{Fr}=0.313) \ldots \ldots \ldots \ldots .62$

35. Vector plot of $\mathrm{w}-\mathrm{v}$ component $(2 \mathrm{x} / \mathrm{L}=1.0, \mathrm{Fr}=0.350) \ldots \ldots \ldots \ldots .63$

36. Vector plot of $\mathrm{w}-\mathrm{v}$ component $(2 \mathrm{x} / \mathrm{L}=1.0, \mathrm{Fr}=0.400) \ldots \ldots \ldots \ldots .64$

37. Vector plot of $\mathrm{w}-\mathrm{v}$ component $(2 \mathrm{x} / \mathrm{L}=1.2, \mathrm{Fr}=0.266) \ldots \ldots \ldots \ldots \ldots 6$

38. Vector plot of $\mathrm{w}-\mathrm{v}$ component $(2 \mathrm{x} / \mathrm{L}=1.2, \mathrm{Fr}=0.313) \ldots \ldots \ldots \ldots 66$

39. Vector plot of $\mathrm{w}-\mathrm{v}$ component $(2 \mathrm{x} / \mathrm{L}=1.2, \mathrm{Fr}=0.350) \ldots \ldots \ldots \ldots .6 .67$

40. Vector plot of $\mathrm{w}-\mathrm{v}$ component $(2 \mathrm{x} / \mathrm{L}=1.2, \mathrm{Fr}=0.400) \ldots \ldots \ldots \ldots .68$

41. Comparison of measured $C_{p}$ and $1-\left(Q_{e} / Q_{\text {ref }}\right)^{2}$

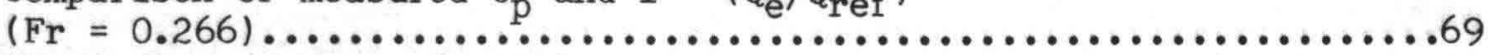

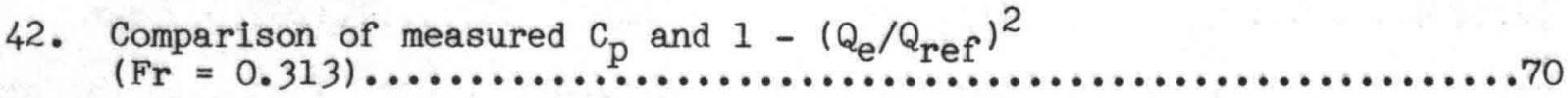

43. Comparison of measured $C_{p}$ and $1-\left(Q_{e} / Q_{r e f}\right)^{2}$
$(F r=0.350) \ldots \ldots \ldots \ldots \ldots \ldots \ldots \ldots \ldots \ldots \ldots \ldots \ldots \ldots \ldots \ldots \ldots \ldots \ldots \ldots \ldots \ldots \ldots \ldots \ldots \ldots \ldots \ldots \ldots \ldots \ldots \ldots \ldots$ 


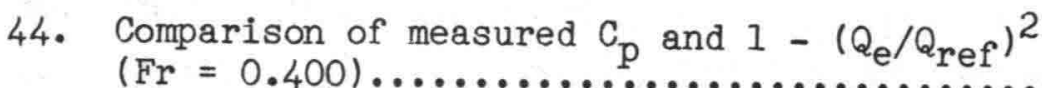

$(\mathrm{Fr}=0.400)$

45. Comparison of displacement thickness along the hull

$(|\mathrm{z} / \mathrm{H}|=0.2)$

46. Comparison of the yaw angles across the boundary layer

$(2 \mathrm{x} / \mathrm{L}=0.8,|\mathrm{z} / \mathrm{H}|=0.2)$

47. Comparison of the yaw angles across the boundary layer

$(2 \mathrm{x} / \mathrm{L}=0.8,|\mathrm{z} / \mathrm{H}|=0.8)$

48. Comparison of the yaw angles across the boundary layer

$(2 \mathrm{x} / \mathrm{L}=0.933,|\mathrm{z} / \mathrm{H}|=0.2)$

49. Comparison of the yaw angles across the boundary layer

$(2 \mathrm{x} / \mathrm{L}=0.933,|\mathrm{z} / \mathrm{H}|=0.8)$

50. Ship $(\mathrm{x}, \mathrm{y}, \mathrm{z})$ and boundary layer $(\xi, \eta, \zeta)$ coordinates............80

51. Measured wave profile and Guilloton's streamline

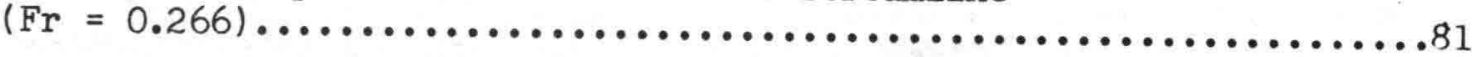

52. Measured wave profile and Guilloton's streamline

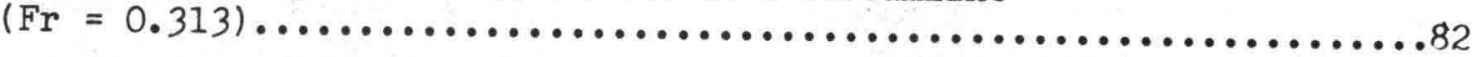

53. Measured wave profile and Guilloton's streamline

$(\mathrm{Fr}=0.350)$

54. Measured wave profile and Guilloton's streamline

$(F r=0.400)$

55. Measured wave profiles on the hull

56. Skin-friction coefficient along the waves.....................

57. Displacement thickness distribution along the waves.............92

58. Momentum thickness distribution along the waves................93

59. Shape parameter (H) distribution along the waves...............94

60. Variation of crossflow angle across the boundary

layer $(\mathrm{Fr}=0.313)$.

61. Variation of shape parameter along the hull for

different Froude numbers.................................96

62. Variation of shape parameter along different

streamlines $(\mathrm{Fr}=0.266)$.

63. Comparison between calculated and measured momentum

thickness $(\mathrm{Fr}=0.350)$. 
64. Comparison between calculated and measured shape parameter $(\mathrm{Fr}=0.350)$

.99

65. Comparison between calculated and measured shape

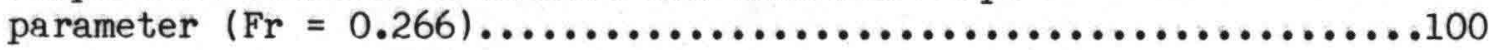

66. Comparison between Michel integral and residuary resistance.......107

67. Comparison between hull-surface distribution and residuary resistance.

.108

68. Comparison between slender-body solution and residuary resistance.

.109

69. Comparison between $\sigma(x, z)$ and $\sigma(x, z)$ (Eq. 37) for

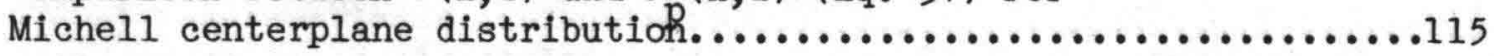

70. Comparison between Michell's thin ship with and without viscous affects ................................118

71. Comparison between slender-body solutions with and without viscous effects.

.119

72. Comparison between hull-surface distributions with

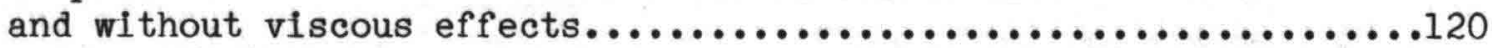

$\mathrm{vi}$ 


\section{LIST OF TABLES}

Table

1. Total-resistance coefficients of Wigley model

(fixed condition)

2. Total-resistance coefficients of Wigley model

(free condition).

3. Measured pressure-distribution coefficient $c_{p} \ldots \ldots \ldots \ldots \ldots . \ldots . \ldots 34$

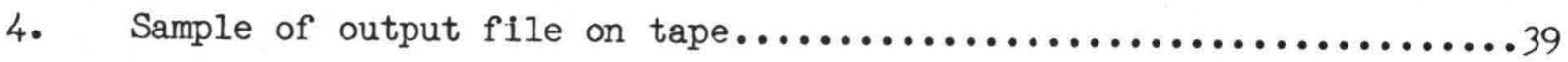

5. Displacement thicknesses of $\mathrm{Eq} .40 \ldots \ldots \ldots \ldots \ldots \ldots \ldots \ldots \ldots \ldots$

6. Comparison between different methods of wave resistance

calculation with and without viscosity effects................122 


\section{LIST OF SYMBOLS}

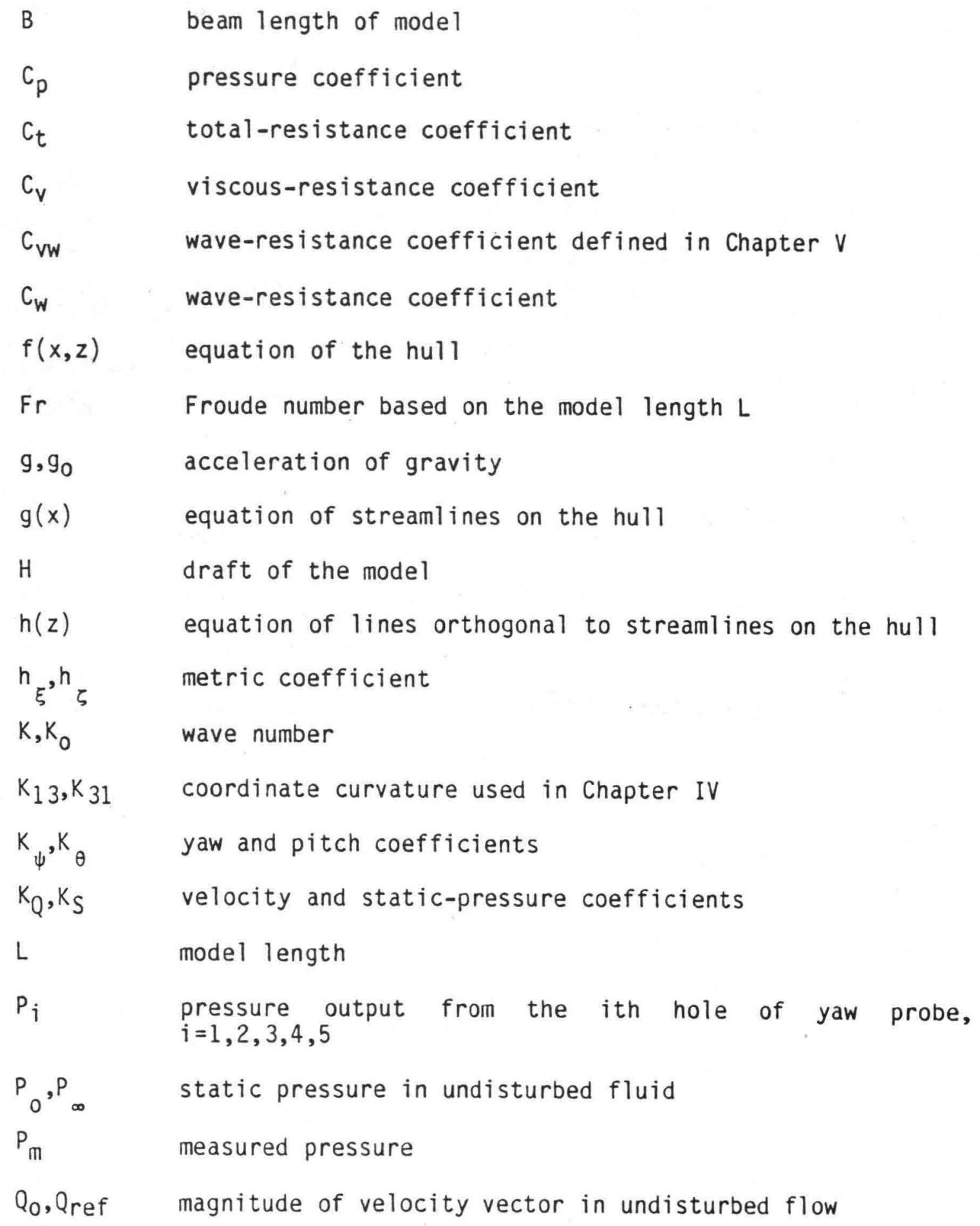




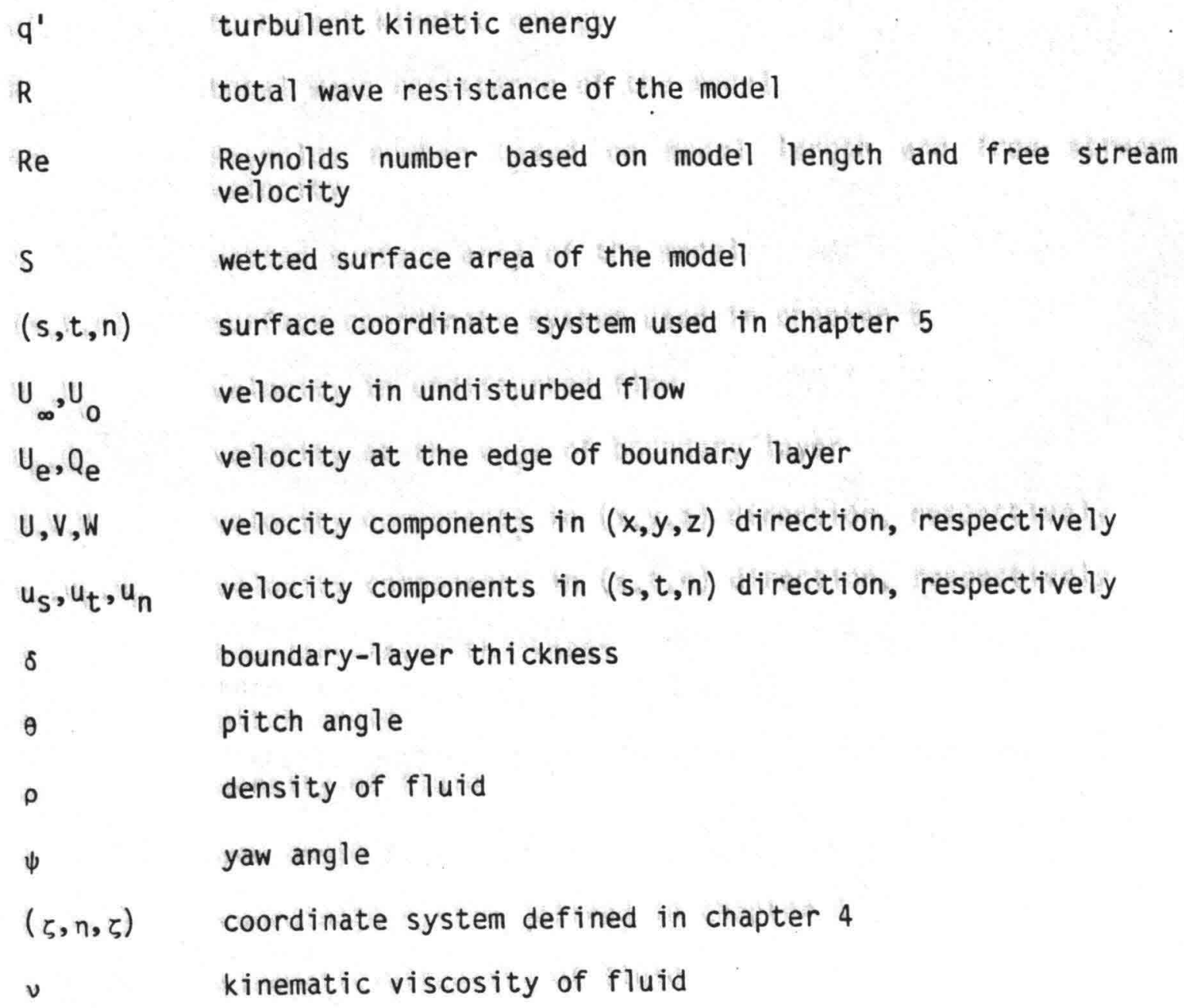




\section{ABSTRACT}

A detailed experimental study has been conducted in a towing tank on a 3.048-meter-long mathematical model, known as the Wigley hull, to study the effects of viscosity on the wavemaking resistance of the ship form. The measurements included total resistance, viscous resistance, pressure distribution, and boundary-layer measurements of the model at zero trim and sinkage. The three-dimensional boundary-layer measurements extend from midship to a distance of 0.1 model lengths downstream of the stern and include the pressure distribution on the body, and three components of the mean velocity measured by means of a five-hole pitot tube. These measurements were carried out for four Froude numbers of $0.266,0.313,0.350$ and 0.400 .

A numerical method, using the small-crossflow approximation of the boundary-layer equations, has been employed to calculate the boundary-layer characteristics along the streamlines on the hull which were obtained by using Guilloton's method. A comparison between calculation and experimental results has been made.

The wave-resistance coefficients of the ship form have been calculated by the Michell integral, using the Michell thin-ship centerplane distribution, a hull surface distribution and a slender-body centerplane distribution. These calculations have been carried out for two different cases, 1) wave resistance calculation without the effect of the boundary layer and wake, and 2) calculations including these effects. These calculations have been carried out only to first order and have been compared with the residuary resistance and the wave-profile resistance.

\section{ACKNOWLEDGEMENTS}

The authors are grateful to Professor V.C. Patel for his advice on boundary-layer calculations and Professor F. Stern for his helpful suggestions. Thanks are also extended to Mr. O.P. Sarda for providing his wind tunnel data. We would also like to thank Mr. Dale Harris, Mr. James Cramer and their staffs for their outstanding support throughout the experimental phase of this study. Special thanks are extended to Mrs. Karen Nall for her excellent typing of this report. 
This study was supported in part by the Office of Naval Research under Contract Nos. N00014-82-K-0069 and N00014-83-K-0136. 
CHAPTER I

INTRODUCTION

\section{Background}

Since Weinblum (1930) introduced Michell's theory (1898) to the field of naval architecture, there have been several attempts to prove its feasibility by comparing the computations according to the theory with measurements in the towing tank. If one plots the wave resistance of a thin ship, as calculated by Michell's integral, versus Froude number, the resulting curve has pronounced humps and hollows. From experiments, however, it is known that the measured wave-resistance curve tends to be much smoother. These discrepancies between the measurements and theory can be attributed to the fact that the basic assumptions of the theory are not fully satisfied. The major assumptions of the derivation of Michell's integral are the thinness of the body, the linearization of the boundary conditions on both the body and the free surface, and the inviscidness of the fluid. Since water is viscous, ship forms are usually not thin, and the boundary conditions are nonlinear, these assumptions are approximate.

In the last decade, with the development of computers of increased speed and capacity, the inviscid problem with the exact hull and free-surface boundary conditions has been investigated by many researchers. Many examples of such calculations are given in the DTNSRDC workshops on ship waveresistance calculations in 1979 and 1983, and the International Symposium on wave resistance in Japan, 1976; and, not surprisingly, the results are in much better agreement with the residuary resistance than those from linearized theory. This has led some researchers to suggest that wavemaking is independent of viscous effects, Newman (1976), and that viscous effects can be neglected in calculating the wavemaking of a ship, Gadd (1976). However, these opinions are not held universally.

There are strong physical reasons for believing that viscosity plays a role in wavemaking. The magnitude of crossflow in the three-dimensional 
boundary layer determines whether or not bilge vortices will be generated near the bow; see Tatinclaux (1970). Another region, where vortex formation and separation have been observed by Tzou (1966) and Chow (1967), is at the approach to a wave crest in the wave profile along the hull, near the stern. In both cases, the pressure field about the hull is modified by these viscous phenomena, so that the wave generated must also be affected. In addition, the displacement effect of the boundary layer and wake modifies the outer potential flow, of which the wave potential is a part. This effect may become especially important toward the stern where the boundary layer is thick, and its displacement thickness significant. Havelock (1948), Wigley (1963), and Milgram (1969) have shown that agreement of the computed wave resistance with the residuary resistance can be definitely improved by modifying the shape of the hull, especially near the stern, in accordance with the displacement thickness of the boundary layer and wake, and applying linearized wave theory to the so-thickened body.

Thus it has been found that agreement with the experimentally-determined residuary resistance can be improved either by calculating the wave resistance more exactly by inviscid-flow theory, or by introducing viscous corrections to linearized wave theory. In an attempt to shed more light on this subject, two investigations were undertaken at the Iowa Institute of Hydraulic Research (IIHR). In the first, the boundary layer and wake of a Series-60 ship model were thickened by roughening the hull surface, and the wave resistance was determined by means of surface profile measurements. The results of Moreno, et al. (1975), and Landweber (1977) showed that the roughening approximately doubled the viscous resistance, and appreciably decreased the wave resistance by 40 percent at a Froude number $\mathrm{Fr}=0.25$, by 50 percent at $\mathrm{Fr}=0.28$, by 13 percent at $\mathrm{Fr}=0.31$, and by 15 percent at $\mathrm{Fr}=0.34$.

In a second investigation, the wave resistance of a thin form, for which the residuary resistance and the linearized wave resistance had been calculated by Weinblum et al. (1952), was recalculated in various ways to determine the relative importance of satisfying more exactly the boundary conditions on the free surface and the hull in the inviscid theory, and of including the effect of the boundary layer and wake. From this investigation, Kang (1978) concluded that, for a very thin form, both the inviscid, nonlinear effects and those of the boundary layer and wake had to be taken into account to obtain good agreement with the residuary resistance. 
In the present study, a more shiplike form has been selected. The selected form is the Wigley parabolic shape, for which a large amount of experimental data and computed characteristics are available, Shearer (1965), Nagamatsu (1979), and Adachi and Hinatsu (1981). There are other advantages in choosing this mathematical form. First, the boundary conditions on the hull surface can be expressed easily; secondly, the Wigley model has no vortex system shed from the bottom because of its sharp keel and, due to its sharp bow, there is no wave-breaking resistance; see Shahshahan (1981). Furthermore, it has been shown, Patel, et al. (1983), that there is no separation zone at the stern. Thus the total resistance may be considered to be composed exclusively of wavemaking resistance and viscous resistance, free of vortex formation or separation, so that with this form, attention can be clearly focused on the interaction between the waves around the hull and the boundary layer.

\section{Review of the Literature}

Since the present study is most concerned with the problem of how ship waves and wave resistance are affected by viscosity, the effect of the nonlinearity of the boundary conditions will not be discussed here. For good reviews of nonlinearity effects refer to Newman (1976), and Ogilive (1976).

Historically, Havelock (1935) was the first to point out the attenuation of the wave due to viscosity. He defined a reduction factor to the wavemaking action which is one at the bow of the ship and decreases toward the stern. Another method for viscosity correction, proposed by Wigley (1937), divided the ship wave resistance into three components, due to the bow wave, the stern wave, and the interference between the bow and stern waves. He defined two correction factors for viscosity which were not independent, and gave empirical expressions which defined them as exponential functions of Froude number. Emerson (1954) considered, instead of the Wigley assumption, that these two factors are independent. Furthermore, he employed another model of the viscosity effect, that is the virtual extension of the stern. A similar idea was adopted by Inui (1957) but with a slightly different form. He attributed one of the reduction factors to nonlinear effects of the finite beam, instead of the "thin ship" of Michell's theory. He called it the self-interference coefficient and gave a semi-empirical relation for it. The aforementioned 
corrections have a common weaknesses, i.e., they lack a rational theoretical background, and they apply only to certain ship forms, and lack universal validity.

The earliest analytical treatment of the problem attributed the effect of viscosity to the displacement of the flow by the thickness of the boundary layer. It was done for the first time in the work by Okabe and Jinnaka (1950), and a practical formula was given by Lavrentiev (1951). Since the displacement thickness is defined only at the body surface, the addition of this displacement to the hull surface results in a body which does not close, since the displacement thickness continues behind the body. A few numerical examples, given by Wigley (1963), showed the effect to be too small to account for the actual phenomena. He then considered an extension of the boundarylayer thickness behind the body in such a way that the virtual streamline forms a closed shape. He found that this modification had a remarkable effect on the wave resistance. At the same meeting, Wu (1963) showed another numerical example but the result did not agree well with the measured values. Arguing that boundary-layer effects on wave formation are appreciable over only a small length of a ship form near the stern, Havelock (1948) suggested that this effect be represented by a virtual extension of the hull shape behind the stern as a cusped wake. He found that the computed wave resistance with this modification was in better agreement with measurements. Many researchers, following Havelock's lead, suggested some sort of wake model behind the body. The proposed wake models are mostly based on the inviscid flow, and may be classified into two groups. These are called the potential-wake models and rotational-wake models according to Maruo (1976), who wrote a thorough review of these methods. Potential-wake models are those in which the effect of displacement of the wake is represented by some distribution of virtual sources or dipoles. The sources that represent the displacement by the wake are called Betz sources. They were considered first by Betz (1925) in his formulation for the experimental determination of viscous drag. The aforementioned Havelock modification of the stern shape corresponds to the addition of these sources. Since the tail point of the modified shape becomes a cusp, this type of wake is called a cusped wake. It is unnecessary, however, that the modified source distribution generate a closed form, and the wake may be of finite width up to infinity downstream. Milgram (1969) employed such an open wake model to calculate the wave resistance of a ship form. 
Rotational wake models assume some distribution of vortices in the wake region, and a general theory for the wave formation by such a vortex distribution was given by Brard (1970). Tatinclaux (1970) carried out a computation of the wave resistance of an ogival strut accompanied by a rotational wake by assuming a volume distribution of vertical line vortices. Beck (1971), assumed another type of rotational wake composed of plane vortex sheets.

The effects of viscosity have also been investigated by Lurye (1968), Wilson (1971), Maruo (1976), and Mori (1978), who linearized the Navier-Stokes equations of the wake and obtained solutions in terms of Oseenlets representing the wake flow. However, since it has been shown by Gadd (1963), and Swain and Landweber (1974) that the wavemaking of vorticity is negligible, and by Preston (1954), Lighthill (1958), and Landweber (1978, 1981) that the irrotational flow equivalent to a boundary layer and wake can be generated by either certain distribution of sources or doublets, or as the flow about a form thickened by the displacement thickness, it appears that the more sophisticated and difficult calculations employing Oseenlets would not yield results for wavemaking essentially different from those employing the displacement thickness. Here "equivalent" denotes that the real flow and the "equivalent" irrotational flow coincide exterior to the boundary layer and wake. In spite of these many efforts, a satisfactory solution of viscous effects on ship wavemaking is not yet available.

\section{The Objectives and Method of Approach}

The problem to be studied is that of the effect of the boundary layer and wake of a ship on its wavemaking resistance. The objectives of this research are, first, to determine the importance of viscous effects on the prediction of ship wavemaking resistance, and second, to incorporate viscous effects into the procedures for computing wavemaking resistance in a way which is physically reasonable and compatible with available procedures for inviscid flow.

In the present study, in accordance with 1979 David W. Taylor Naval Ship Research \& Development Center (DWRNSRDC) workshop recommendations, the Wigley ship model will be restrained so that there is no sinkage or trim, and its resistance and flow characteristics will be measured in a towing tank. The measurements include total resistance, viscous resistance by means of a wake survey, the pressure distribution on the hull, and velocity profiles in the 
three-dimensional turbulent boundary layer. Using these data, the boundarylayer characteristics of the hull will be calculated. These results will be compared with a numerical solution of the three-dimensional boundary layer along the streamlines on the hull. Guilloton's method (1951) will be employed to obtain the streamlines on the hull.

The next step will be the calculation of the wave resistance of the model, by using both Michell's thin-ship and a slender body centerplane source distribution. In this part, we shall consider two different cases, 1) waveresistance calculation without the effect of the boundary layer and wake, and 2) calculations including the effect of the boundary layer and wake. These calculations will be carried out only to first order and will be compared with the residuary resistance.

\section{Plan of the Report}

Chapter II contains a description of experimental details, including the instrumentation and data-acquisition systems. The results are presented and discussed in Chapter III. Numerical procedures for boundary-layer calculations and a comparison of the results of measurements with the calculated values are discussed in Chapter IV. In Chapter V, wavemaking-resistance calculations with various refinements are presented and compared with experimental values. Chapter, VI, contains the conclusions and the recommendations for future work. 
CHAPTER II

EXPERIMENTAL EQUIPMENT AND PROCEDURES

\section{The Model}

All experiments were performed in the Iowa Institute of Hydraulic Research (IIHR) towing tank which has been described by Martin (1958). The towing tank is $91.44-\mathrm{m}$ long, 3.048-m wide and 3.14-m deep. The ship model employed in this study was a member of the series of mathematical models with parabolic sections and waterlines, introduced by Wigley (1942). This form is characterized by sharp edges at the bow, stern and keel, and is defined by

$$
\begin{gathered}
|Y|=\frac{B}{2}\left[1-\left(\frac{2 x}{L}\right)^{2}\right]\left[1-\left(\frac{z}{H}\right)^{2}\right] \\
-\frac{L}{2} \leq x \leq \frac{L}{2}-H \leq z \leq 0
\end{gathered}
$$

in a Cartesian $(x, y, z)$ coordinate system with the origin at midship on the undisturbed free surface, increasing value of $x$ opposite to the direction of the ship's motion, and the z-axis directed vertically upward. Here $L$ is the length of model, $B$ is its beam and $H$ the draft. The selected form had a length of $3.048-\mathrm{m}$, and parametric values of $\frac{B}{L}=0.100$ and $\frac{H}{L}=0.0625$. The block coefficient of the model is $C_{B}=0.444$ and its wetted surface area is $1.381 \mathrm{~m}^{2}$. The model was equipped with three rows of pressure taps, from midsection to the stern on the portside. These rows were at the levels $|\mathrm{z}|=$ $0.635,3.81$, and $11.43 \mathrm{~cm}$. For turbulence stimulation along the hull, a row of plastic studs of $3.2 \mathrm{~mm}$ diameter, $1.6 \mathrm{~mm}$ height and $9.5 \mathrm{~mm}$ spacing was fitted on the model at $15.2 \mathrm{~cm}$, five percent of the model length, from the bow. Figure 1 shows the Wigley model. With the towing arrangement used, the model was restrained in both trim and sinkage for all the experiments, except the total-resistance measurements, which were performed for both fixed and free conditions. 


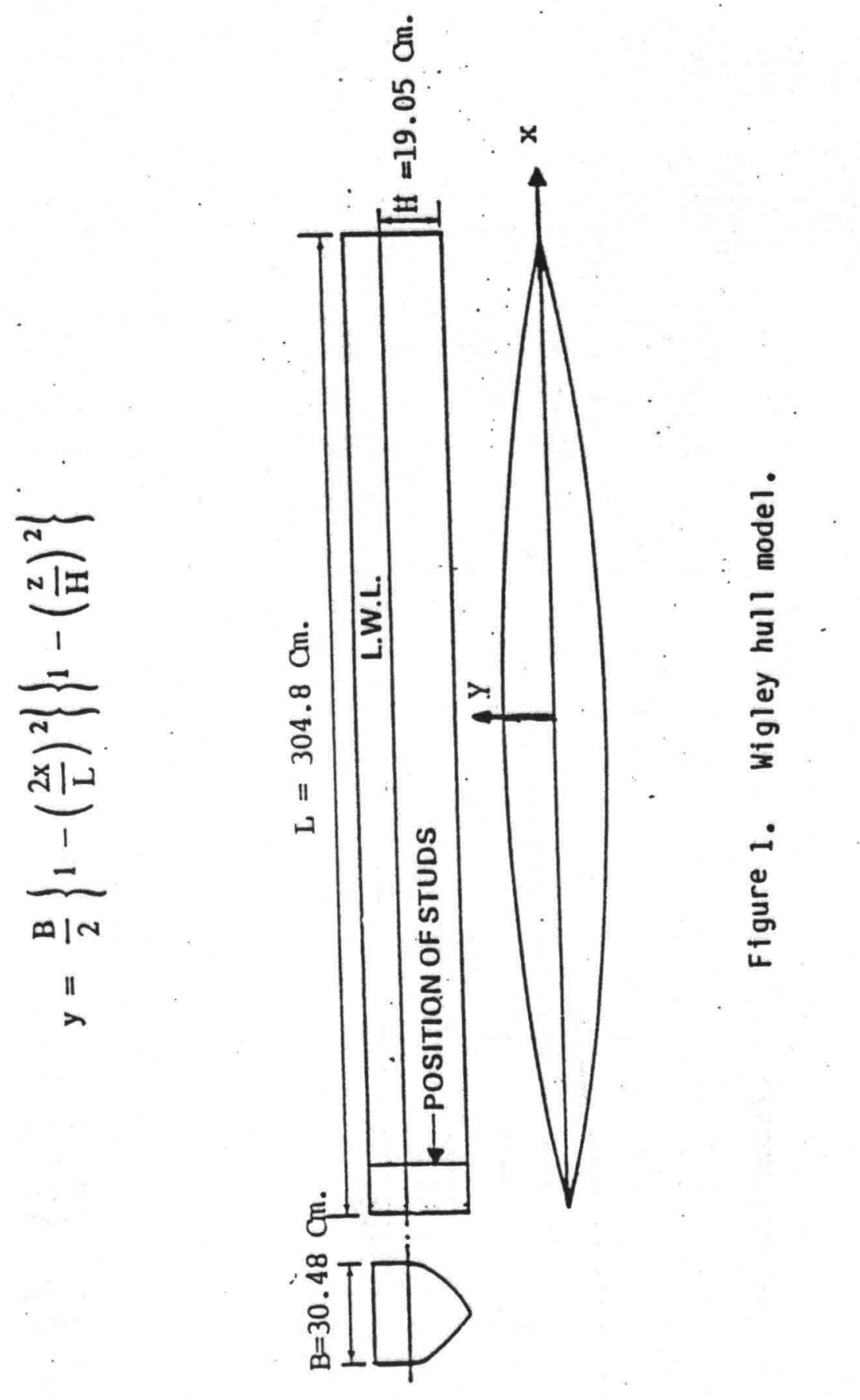




\section{Instrumentation}

A five-hole yaw probe was used to obtain the direction and magnitude of the velocity in the three-dimensional boundary layer and wake. The probe was L-shaped with a shorter portion of $75 \mathrm{~mm}$ and a longer section of $300 \mathrm{~mm}$. It was made of five stainless-steel tubes of one millimeter inner diameter, with overall diameter of $4.2 \mathrm{~mm}$. Since it has been shown by Bryer et al. (1955) that, for boundary-layer measurements in general, a sharp-edged probe is more satisfactory than a rounded one, as it is less affected by changes of Reynolds number, the five-hole probe was fabricated with a sharp tip. For moving the probe, a traverse mechanism, which could move the probe in three directions of a Cartesian coordinate system, was designed and fabricated in the IIHR shop. The lateral and vertical movements were measured by counters with the minimum counts of 2.00 and $2.05 \mathrm{~mm}$, respectively. The pressure tubes from the fivehole yaw probe were connected to five differential-type pressure transducers, which were mounted on a rigid fixture on the traverse. In order to obtain the optimum sensitivity in measurements, these transducers were of different ranges. The center hole of the probe was connected to a pressure transducer with a range of $0.02 \mathrm{~N} / \mathrm{m}^{2}(0.3 \mathrm{psid})$, and the other holes were connected to pressure transducers with a range of $0.01 \mathrm{~N} / \mathrm{m}^{2}(0.15 \mathrm{psid})$. These pressure transducers formed a component of a strain-gauge bridge. The out-of-balance voltages from these bridges, which are proportional to the pressure sensed by transducers, then were sampled, digitized and recorded by the data acquisition system.

In the total-resistance measurements, the model was attached to the dynamometer of the towing carriage. A force transducer with a range of \pm 4.54 $\mathrm{kgf}$ was employed to measure the total resistance of the model via a frequency counter.

\section{Calibration}

\subsection{Force Transducer}

The force transducer was calibrated against dead weights. This calibration was checked before and after each set of total resistance measurements, in orders of both decreasing and increasing weights. The calibration was found to be linear and very stable. 


\subsection{Pressure Transducer}

The pressure transducers were calibrated against an alcohol manometer with a least count of $0.025 \mathrm{~mm}$ of alcohol. The calibrations were performed at the experiment site using the actual connecting cables and data-acquisition system. During the course of measurements, three or four points of the calibration curves were checked every other day. The calibration curves were found to be linear and very stable.

\subsection{Calibration of 5-Hole Yaw Probe}

The five-hole yaw probe was calibrated in an open-throat wind tunnel. The probe was placed in the working section of the tunnel on an assembly that allowed it to be yawed as well as pitched about the flow direction at any prescribed angle. A total-head tube, mounted at the same longitudinal location but a small spanwise distance away from the yaw probe, served as the reference. The five-hole probe was connected to a single differential pressure transducer through a scanivalve; see Cramer (1984). The static pressure tap of the transducer was connected to the static pressure of the total-head probe. Since it has been shown by Rajaratnam and Muralidhar (1968) that the effect of Reynolds number on calibration is insignificant at air speeds greater than $12 \mathrm{~m} / \mathrm{sec}$, the calibration was carried out at an air speed of 17.554 meters per second.

If the uniform flow is assumed to be along the $x$-axis, the yaw angle $\psi$ and pitch angle $\theta$ are defined as shown in Figure 2. This figure also shows the numbering scheme used for the five-hole probe. Let $P_{1}, P_{2}, P_{3}, P_{4}$, and $P_{5}$ be the pressures sensed by the holes numbered one through five, respectively. Then it is evident that the holes numbered 2 and 4 are primarily sensitive to yaw and the holes numbered 3 and 5 are sensitive to pitch. Based on this, and extending the concept used by Ramaprian et al. (1978) with a three-hole yaw probe, the following coefficients are defined:

similarly for pitch angle: $\mathrm{K}_{\psi}=\frac{\mathrm{P}_{2}-\mathrm{P}_{4}}{\mathrm{P}_{1}-\frac{1}{2}\left(\mathrm{P}_{2}+\mathrm{P}_{4}\right)}$

$$
K_{\theta}=\frac{P_{3}-P_{5}}{P_{1}-\frac{1}{2}\left(P_{3}+P_{5}\right)}
$$


Furthermore, if $Q$ is the magnitude of the stream velocity and $P_{S}$ the local static pressure at the probe, the velocity coefficient is defined as

$$
K_{Q}=\frac{P_{1}-\frac{1}{4}\left(P_{2}+P_{3}+P_{4}+P_{5}\right)}{\frac{1}{2} \rho Q^{2}}
$$

and finally the static-presure coefficient is given by

$$
K_{S}=\frac{P_{1}-P_{S}}{\frac{1}{2} \rho Q^{2}}
$$

where, in the calibration process, $\mathrm{P}_{\mathrm{S}}=0$.

The probe was calibrated for the range of $-35^{\circ} \leq \psi \leq 35^{\circ}$ and $-35^{\circ} \leq \theta \leq$ $35^{\circ}$ at 5 -degree steps in yaw and pitch angles. The aforementioned nondimensional calibration coefficients were calculated from these measurements. The nondimensional yaw coefficient $K_{\psi}$ was plotted against the yaw angle $\psi$ for different pitch angles. Similarly, $K_{\theta}$ was plotted against $\theta$ for different yaw angles. Also $\mathrm{K}_{Q}$ and $\mathrm{K}_{\mathrm{S}}$ were plotted against $\theta$ for different values of $\psi$. See figures 3 through 6 .

Later, the five-hole yaw probe was calibrated dynamically in the towing tank at zero angles of yaw and pitch; see Figure 7.

\section{Data Acquisition System}

At the heart of the data-acquisition system is an HP-1000 E-Series minicomputer which was connected to the experiment site through a 16-channel Preston analog-to-digital converter. The carriage velocity and pressure data were sampled simultaneously by this system. A block diagram of the system is shown in Figure 8. A generator linearly converts carriage speed to frequency, which is then transformed into voltage by a frequency-voltage converter. The sensed pressures from the 5-hole yaw probe are converted to voltages by pressure transducers and amplified by signal conditioners. These voltages are sampled simultaneously through six different channels of the analog-to-digital converter, digitized, and then read and stored by the computer. 


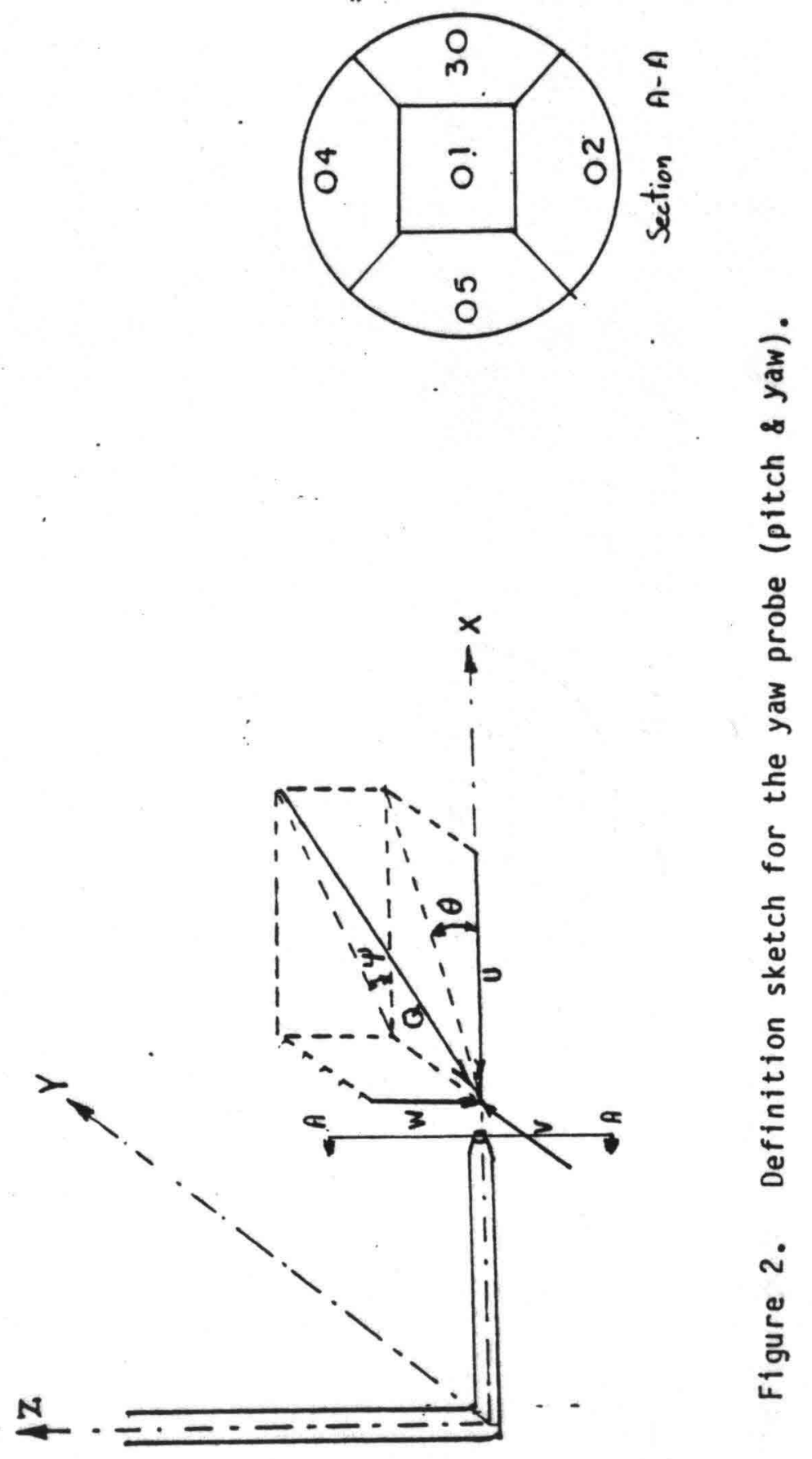




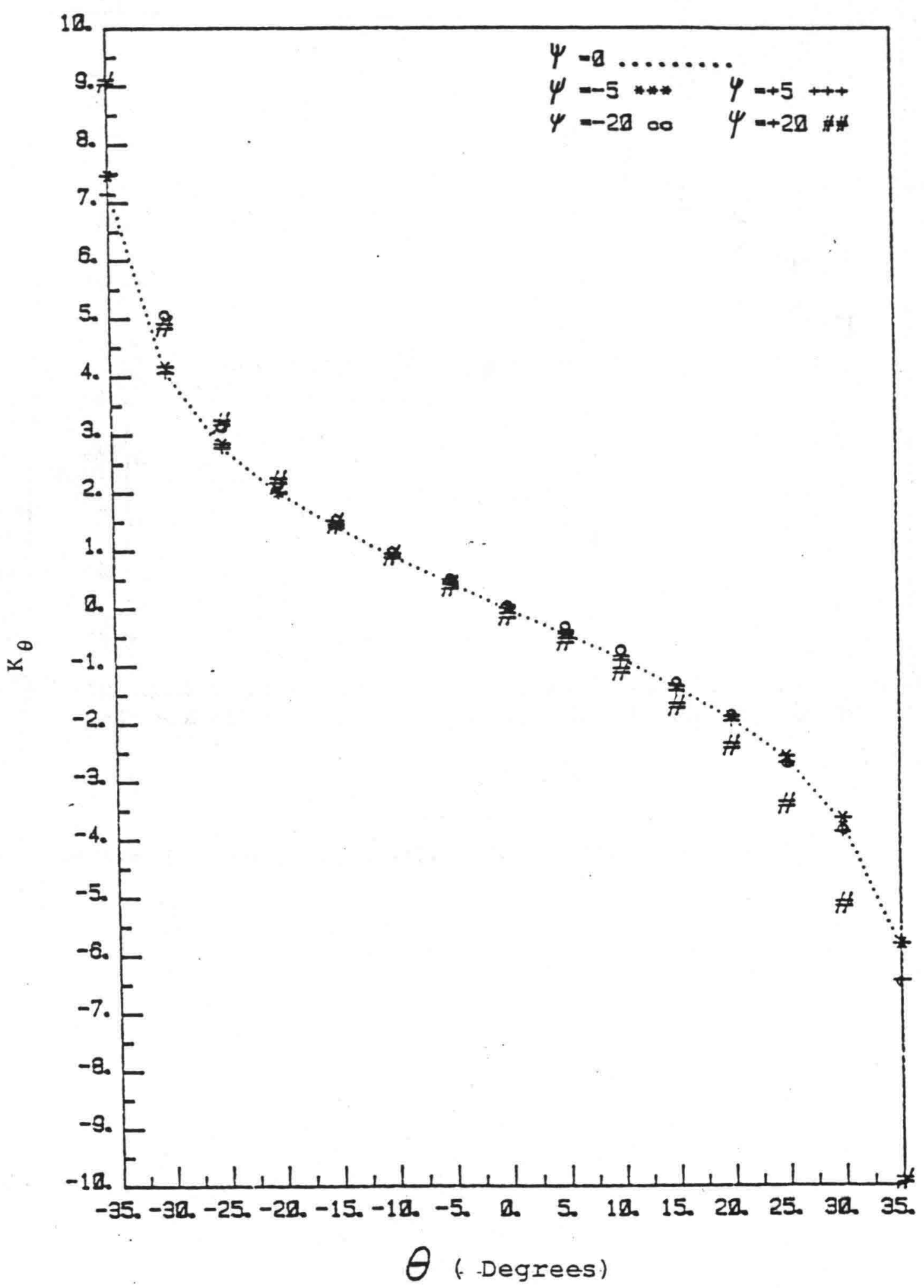

Figure 3. Yaw probe calibration curves for pitch angles. 


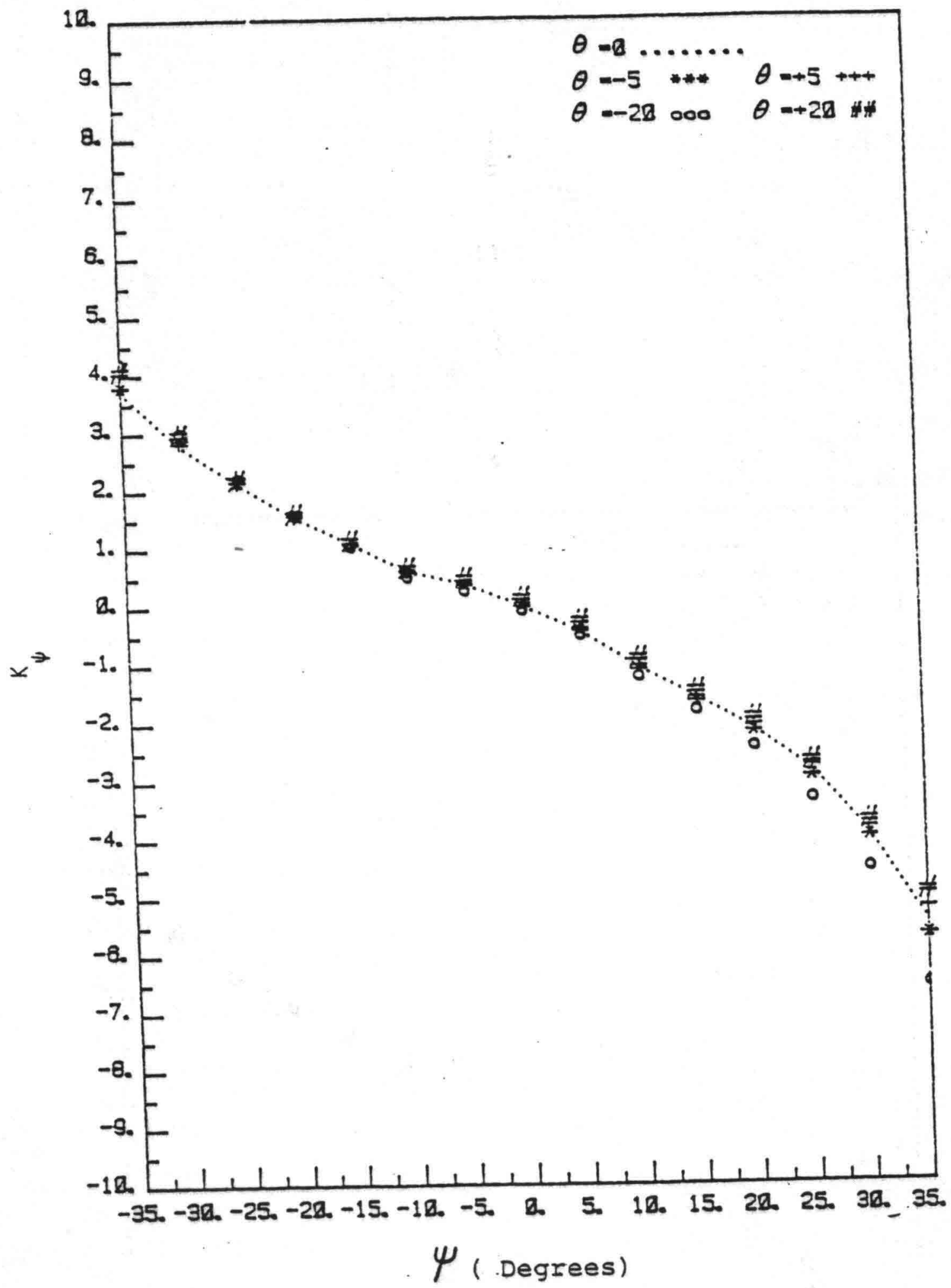

Figure 4. Yaw probe calibration curves for yaw angles. 


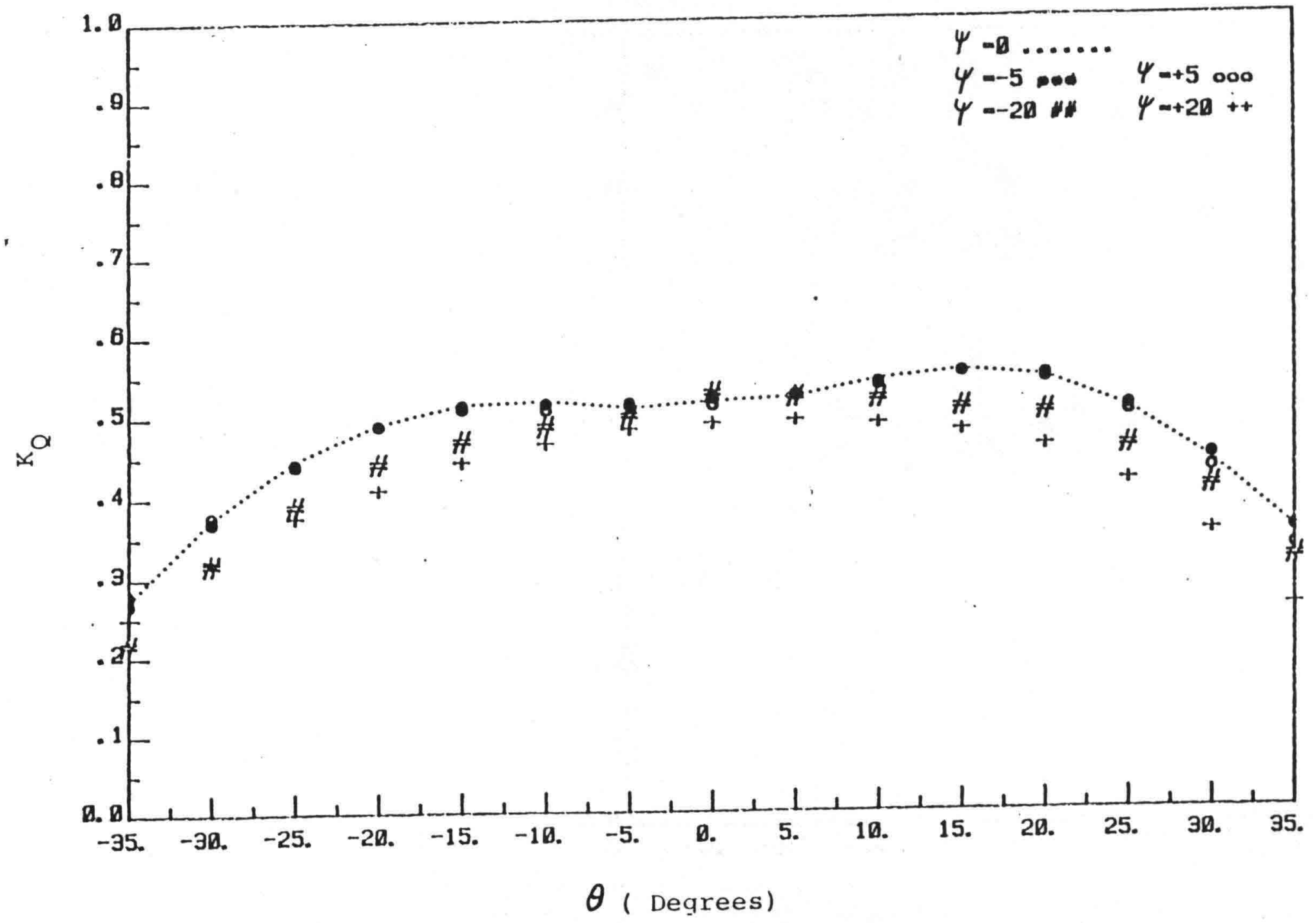

Figure 5. Yaw probe calibration curves for the magnitude of velocity.. 


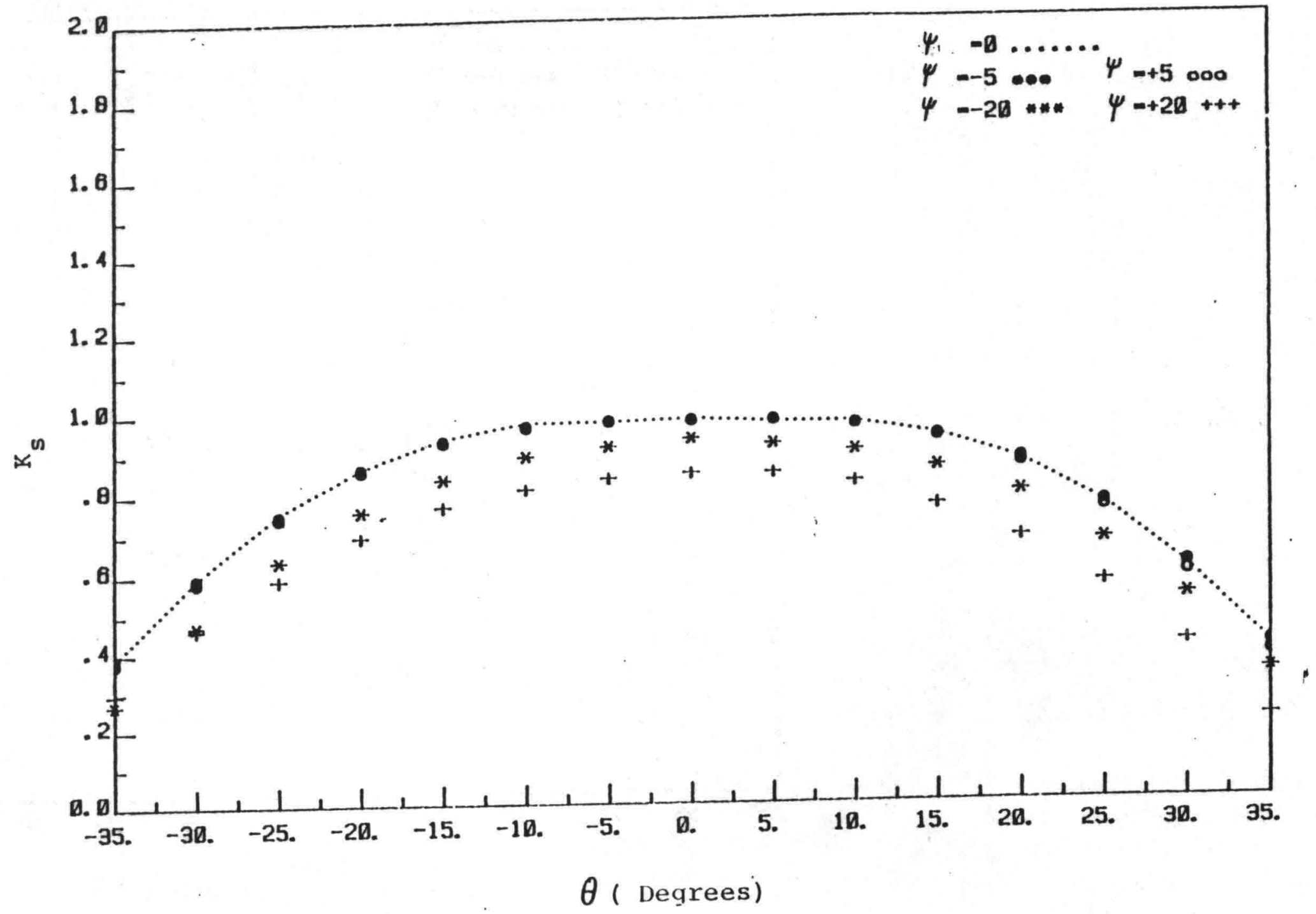

Figure 6. Yaw probe calibration curves for the local static pressure. 


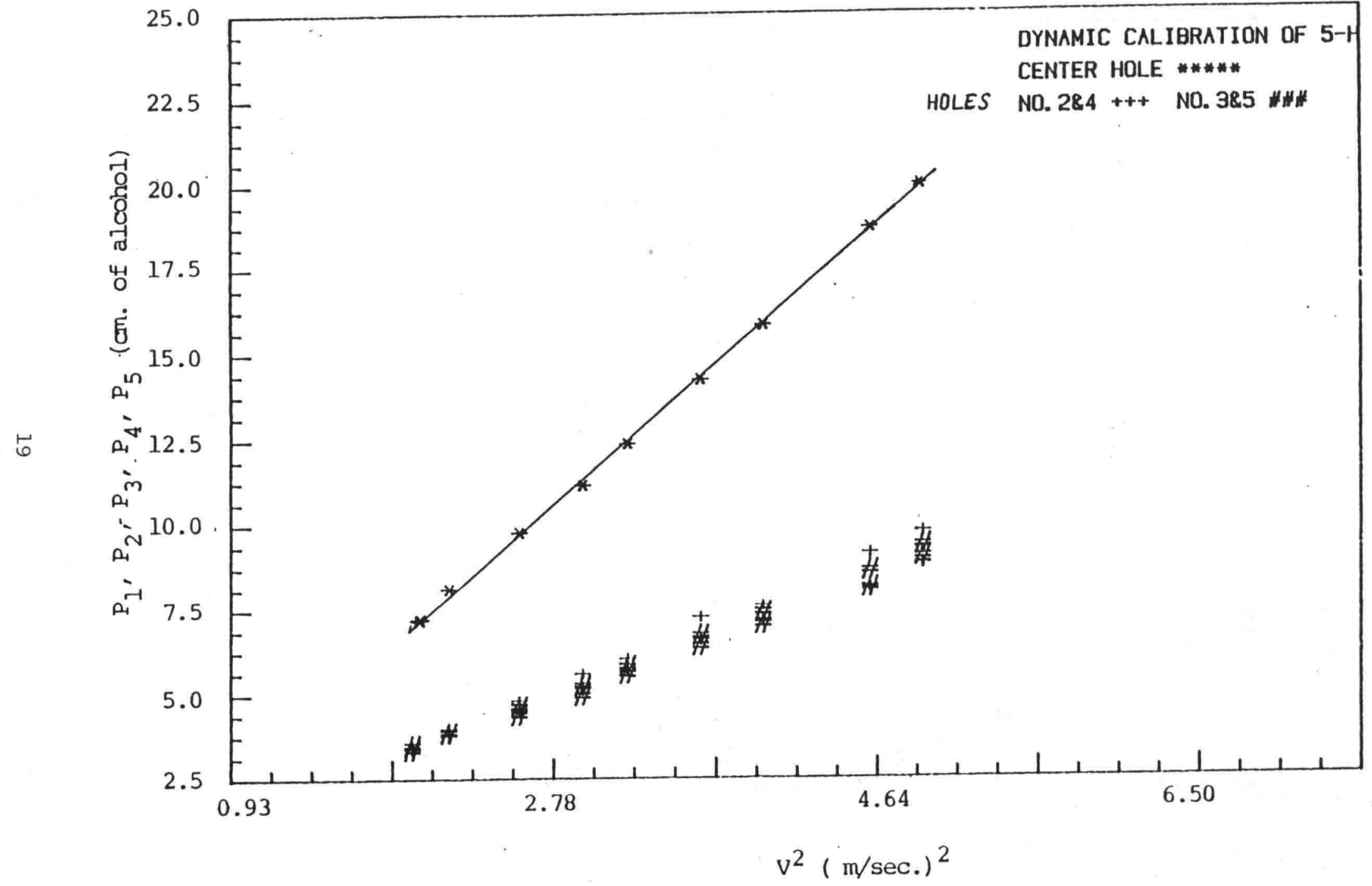

Figure 7. Dynamic calibration curves for five-hole yaw probe. 


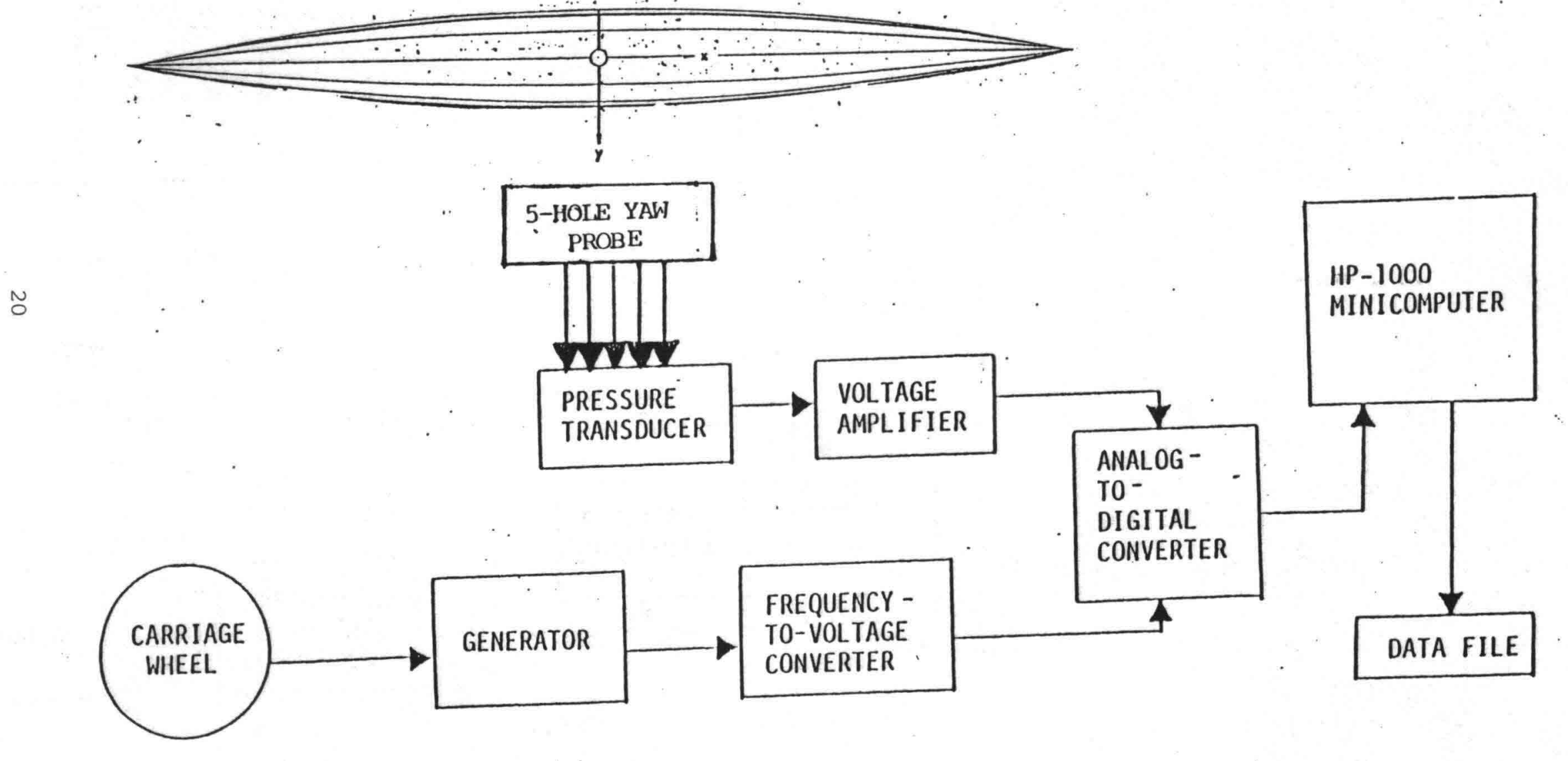

Figure 8. Data-acquisition system for the carriage speed and boundary layer and wake measurements. 


\section{Experimental Techniques}

\subsection{General Remarks}

The model was attached under the carriage to the dynamometer. For the free condition, there was only one point of attachment and two guides were used at the bow and stern to prevent lateral movements of the model. The model alignments were checked by levels and also by measuring the distances of the bow and stern from the wall of the tank to make sure that the model was not yawed or pitched with respect to the direction of motion. For the fixed condition, a parallelogram arrangement was used instead of guides at the bow and stern to prevent the model from deviating from its initial sinkage and trim.

Since a system of water and air was used to transmit pressure to the pressure transducers, there was the problem of air-bubble trapping in the connecting tubes. This was avoided by cleaning the tubes before each experiment by compressed air and then adding a drop of photoflo fluid to each tube.

The Analog-to-Digital (A/D) converter of the data-acquisition system can read \pm 10 volts and it is recommended that the entire range be used to reduce cable noise at low voltages caused by the long cable length of 210 meters connecting the experiment site to the A/D converter. For this reason, a gain of 600 was used to magnify the low voltages from the pressure transducers. Cable noise was found to affect the measured pressure by $\pm 0.57 \times 10^{-5} \mathrm{~kg} / \mathrm{cm}^{2}$ in the first four channels, and by $\pm 0.88 \times 10^{-5} \mathrm{~kg} / \mathrm{cm}^{2}$ in the next two channels of the A/D converter. The relative magnitude of these noise levels to the measured quantities was found to be less than 0.1 percent.

For tests in the towing tank, a time interval of 15 minutes between carriage runs was found to be necessary. This was determined by injecting dye beneath the free surface and studying the motion of dye filaments. It was observed that even after 15 minutes the water has a velocity about 0.2 $\mathrm{cm} / \mathrm{sec}$. This gives an error of 0.12 percent in the measured velocity.

\subsection{Total-Resistance Measurements}

Initially, the mechanical system used to tow the model introduced large fluctuations in the reading of data. This was due to speed oscillations of the speed control at low speeds, which resulted in inertial forces on the 
model of the same order of magnitude as the resistance to be measured. Some preliminary tests showed that these oscillations are of high frequency, so that, by using a low-pass filter, this problem was corrected. Recorded readings with and without the electronic filter showed a marked improvement of the system. For resistance measurements, the output signal of the transducer was averaged over a time interval of 10 or 5 seconds depending on the speed of that particular run. At least two time averages of the signal were recorded during each run.

Total resistance measurements were performed for a range of Froude numbers up to 0.400 , for free and fixed conditions. These results are shown in Tables 1 and 2 .

\subsection{Boundary Layer and Pressure Measurements}

Initially, it was attempted to measure the pressure distribution and boundary layer on the Wigley hull via a scanivalve. It was found, however, that the duration of a run in the towing tank was not long enough for pressure readings of a five-hole pitot tube to attain their asymptotic values. Hence, the scanivalve was abandoned, and five pressure transducers were directly connected to the 5-hole pitot tube. Pressure distribution on the hull was also measured by direct connection of transducers to the pressure taps. With this arrangement, mean-flow measurements were taken in ten streamwise locations from midship to the stern. At each section the boundary-layer measurements were carried out for five waterlines. Figure 9 shows the measuring positions on the Wigley hull. These measurements were continued into the wake of the model for four streamwise sections and five girthwise locations at each section. The boundary-layer and wake measurements were made at four different speeds, corresponding to the Froude numbers 0.266, 0.313, 0.350, and 0.400. For each Froude number, since the model velocity was not exactly the same for different runs, the pressure readings of the 5-hole pitot was corrected to the speed corresponding to the Froude number by the following relation:

$$
P_{m}=\left(\frac{V_{c}}{V_{c}^{\prime}}\right)^{2} P_{m}^{\prime} \quad(m=1,5)
$$

where $V_{c}^{\prime}$ is the carriage speed corresponding to the measured value of $\mathrm{P}_{\text {im }}$ and $\mathrm{V}_{\mathrm{C}}$ is the speed corresponding to the desired Froude number. 
TOTAL RESISTANCE COEFPICIENT CE (FIXED CONDITION $T=18.3 \mathrm{C}$ )

\begin{tabular}{|c|c|c|c|}
\hline ER & $C t$ & $\boldsymbol{F} \mathbf{R}$ & Ct \\
\hline $\begin{array}{l}0.100 \\
0.120 \\
0.140 \\
0.160 \\
0.180 \\
0.200 \\
0.2200 \\
0.240 \\
0.265 \\
0.280\end{array}$ & $\begin{array}{l}0.00440 \\
0.00434 \\
0.00431 \\
0.00424 \\
0.00425 \\
0.00429 \\
0.00432 \\
0.00450 \\
0.00451 \\
0.00464\end{array}$ & $\begin{array}{l}0.300 \\
0.315 \\
0.330 \\
0.340 \\
0.350 \\
0.360 \\
0.370 \\
0.380 \\
0.390 \\
0.400\end{array}$ & $\begin{array}{l}0.00496 \\
0.00511 \\
0.00503 \\
0.00500 \\
0.00500 \\
0.00500 \\
0.00505 \\
0.00520 \\
0.00540 \\
0.00560\end{array}$ \\
\hline
\end{tabular}

Table 1. Total resistance coefficients of Wigley model (fixed condition). 
TOTAL RESISTANCE COEFFICIENT CE

(FREE CONDITION $T=13.3 \mathrm{C}$ )

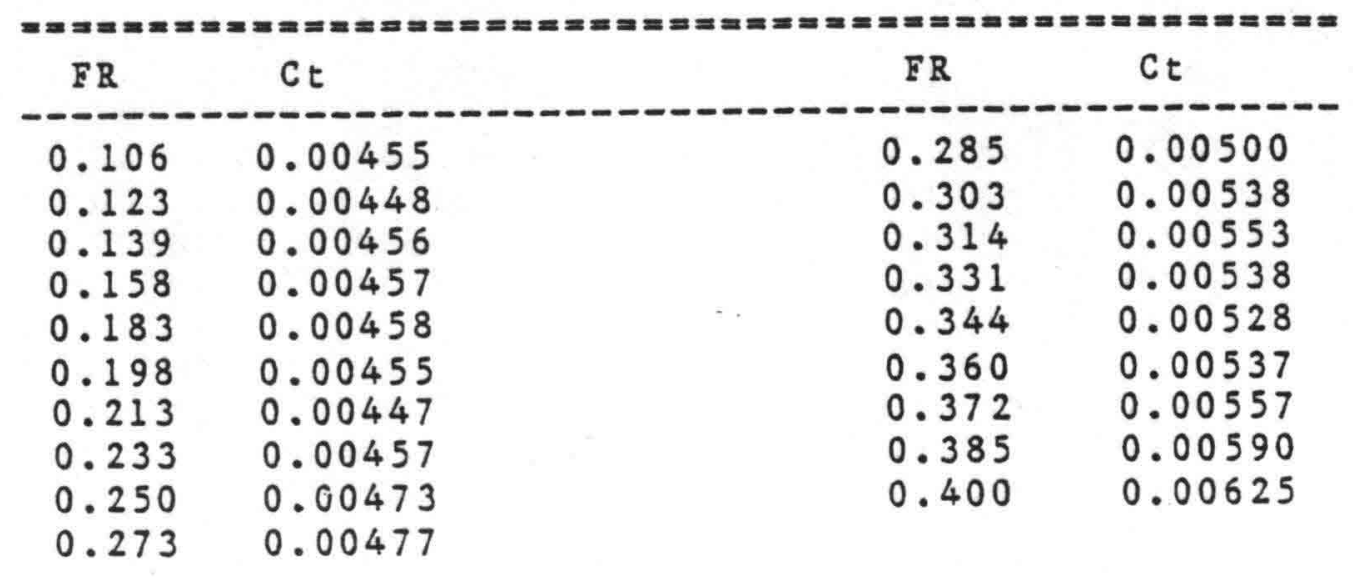

Table 2. Total resistance coefficients of Wigley model (free condition). 


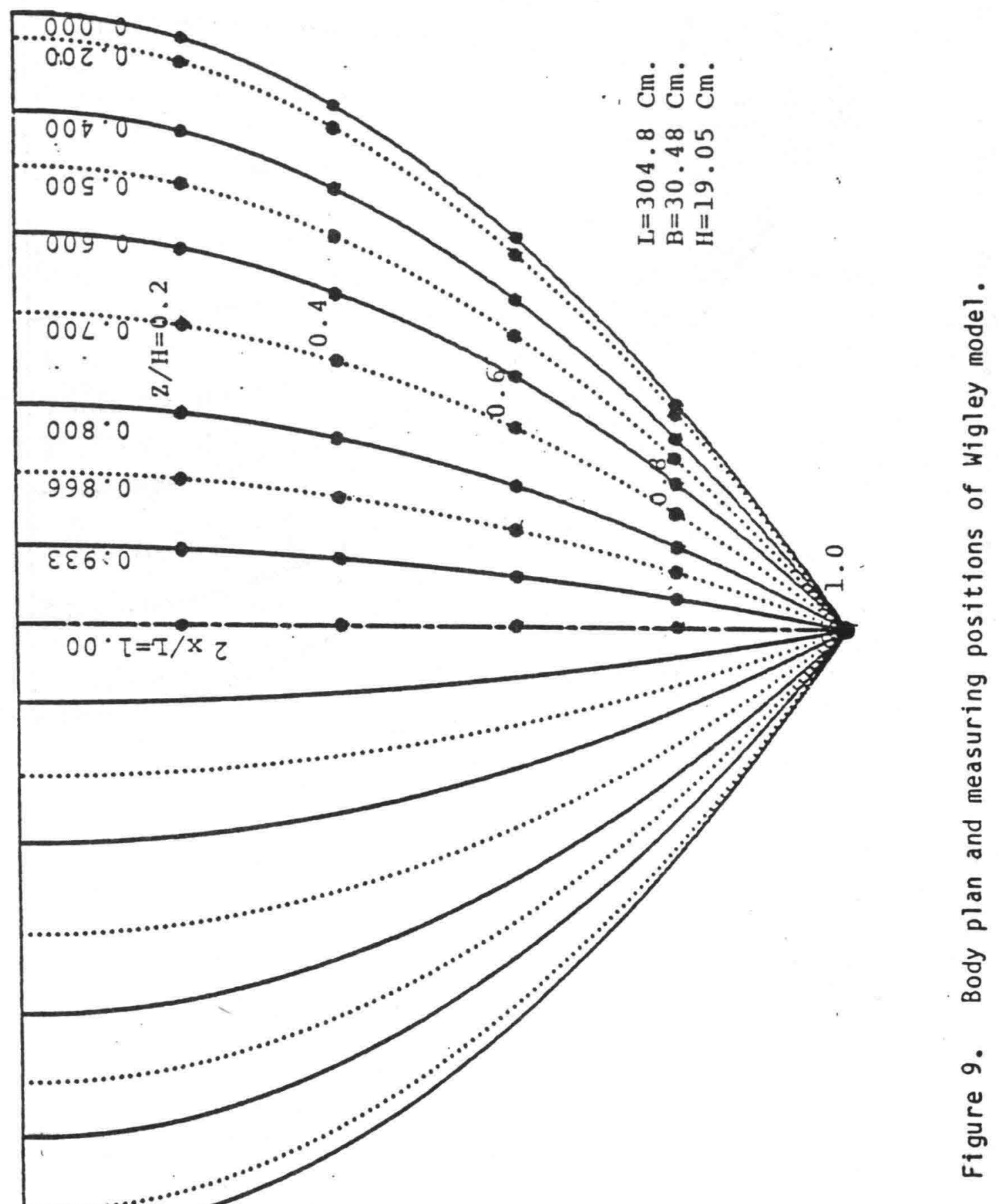


CHAPTER III

RESULTS AND DISCUSSION

\section{Error Analysis of Measurements}

Before discussing the results obtained from the measurements of velocity field and pressure distribution around the hull, it may be necessary to analyze the error involved in the measurements in order to have a better understanding of the results. Generally, the error involved in the experiments can be divided into two groups: physical errors, which are encountered during the course of measurements, and numerical errors, which occur in processing the resulting data. In the following, a brief description of the sources of physical and numerical errors, together with their order of magnitude, is given.

\subsection{Physical Errors}

The most common sources of error in measuring either dynamic or static pressure in the present study are the following:

i) Alignment error: The yaw angle of the 5-hole yaw probe was checked against a level, and its pitch angle was checked against a parallel line by eye. This may give about a one-degree error in pitch-angle alignment. Meriam and Spaulding (1935), have shown that the resulting error in measuring dynamic pressure, for one-degree misalignment, is very much less than one percent of actual value.

ii) The influence of probe support: The pressure gradient associated with the curvature of flow lines around probe supports was avoided by placing the probe at least about 15 diameters from its support, which is three times the recommended five-diameter distance. Thus this source of error was completely avoided. 
iii) Wa11-proximity effects: When a total-head probe approaches a wall, it is shown by MacMillen (1956) that the streamlines are deflected by the probe-wall interaction. Such an interaction results in an error of up to 10 percent in dynamic pressure measurement when the distance from the axis of the probe to the wall is less than 1.5 probe diameters, i.e., in the present measurements, the first two measured points of boundary layer at each location are affected by this error.

iv) Pitot-tube displacement effect: It is shown experimentally by Young and Mass (1936), and theoretically by Hall (1956), that a pitot tube whose axis is at a fixed point $A$, measures the total pressure at a point B a short distance away from A. This distance which is known as displacement effect of the pitot tube due to its finite size, in general is a function of the shape of the tube, its diameter, kinematic viscosity and velocity of the fluid. For the 5-hole probe used in the present study, the ratio of the displacement effect to the probe diameter is approximately between 0.1 for $\mathrm{Fr}=0.400$ up to 0.14 for $\mathrm{Fr}=$ 0.266. This means that the shift in measured points due to displacement effect of five-hole pitot tube is less than $0.5 \mathrm{~mm}$.

v) Turbulence effects: Turbulence may affect the reading of the pitot tube in two ways. Firstly, the r.m.s. value of the fluctuating velocity components may directly affect the calibration of the pitot tube. However, this is generally believed to be small and of secondary importance. Of primary importance is the direct effect of turbulent velocity components on the total-head tubes, which causes the sensed value of mean total pressure to increase. This can be shown by a simple analysis of the measurements of a pitot tube:

$$
\begin{aligned}
P_{m} & =P+\frac{1}{2} \rho \bar{Q}^{2} \\
& =P+\frac{1}{2} \rho q^{2}+\frac{1}{2} \rho \bar{q}^{\prime 2}
\end{aligned}
$$

Unfortunately, in the present study, the turbulence quantities were not measured and an exact estimate of the turbulent effect is not possible. However, it has been shown by Goldstein (1936), and Walsche \& 
Garner (1960), that the pitot tube responds mainly to the longitudinal component of turbulent velocity, especially when the resultant mean velocity does not make too large a pitch or yaw angle with the axis of probe. So one can simply replace the total turbulent energy term $\bar{q}, 2$, by the longitudinal component $\bar{u}^{2}$. For the usual turbulence intensity encountered in the water flows the turbulence effect on the probe is small; e.g., for a turbulence intensity of 20 percent which is too high for practical situations, the pressure reading of the yaw probe will increase by only 2 percent.

vi) Hole geometry for static-pressure taps: According to Shaw (1960), an 'ideal' tap geometry is a small circular hole of less $1 / 4-\mathrm{mm}$ diameter drilled perpendicular to the surface on which the pressure is to be measured, with the corner of the hole perfectly sharp and squared off. Any departure from this geometry will introduce errors. Errors with a practical-sized hole occur because of flow in and around the hole opening. In the present study, the diameter of the pressure taps was one millimeter. This should introduce an error of less than one percent of $\frac{1}{2} \mathrm{U}_{0}^{2}$ compared with a hole of $\frac{1}{4} \mathrm{~mm}$. Nonperpendicularity of the pressure taps with the wall will also introduce some error, but this error for small deviation is of secondary importance and can be neglected. Thus the overall-error estimation due to the geometry of the pressure taps in this case is less than one percent.

\subsection{Numerical Errors}

As already mentioned in Chapter II, a computer program was developed in order to obtain the velocity vector in the boundary layer from the five-hole yaw-probe measurements. In the numerical scheme used, polynomials of the third degree were fitted by the method of least squares to the calibration results, and values at desired points were obtained by linear interpolation. To obtain an estimate of the errors due to the polynomial fittings and interpolations, a numerical study was conducted. A series of calibration data with zero pressure coefficients and exactly known pitch and yaw angles was used as an input to the program. The output for this test showed that, for small angles of yaw and pitch between zero and \pm 5 degrees, the error was less than 0.4 degree. For angles between \pm 5 and \pm 15 degrees, the error varied between 
0.5 to 1.2 degrees. The error increased to 2 degrees for pitch or yaw angles between 15 and 25 degrees. For larger pitch and yaw angles between 25 and 35 degrees, the error was between 2.2 and 3.5 degrees. These numerical errors are indeed very small, first because, for most part, the flow around the Wigley hull can be classified as one with small cross-flow, and the measurements showed that the yaw or pitch angles are less than 10 degrees over most of the hull, except near the stern where they can be as high as 15 degrees. Secondly, since these angles are used in trigonometric functions to calculate the velocity components, these numerical errors become even less appreciable. This test showed that the overall numerical error in obtaining the velocity components is less than two percent. For the five-hole yaw-probe, measurements of pressure coefficients, however, appreciable errors were found. The test result showed that, for the pitch and yaw angles between zero and 15 degrees, the pressure coefficients have values ranging from 0.006 up to 0.02 , in contrast to the actual value of zero. These variations, at the first glance, may seem small enough, but they can have a significant effect, especially near the midship, where the actual pressure coefficients are small and of the same order as the numerical errors. For this reason it is recommended that the 5-hole probe measurements of pressure coefficient, especially around the midship, be disregarded.

From the discussion in this section, it may be concluded that the overall physical and numerical errors, except for five-hole probe measurements of pressure coefficients, are small, and the present measurements are of acceptable accuracy within an uncertainty of a few percent. It should be noted that none of the data was corrected for the aforementioned physical or numerical errors. 


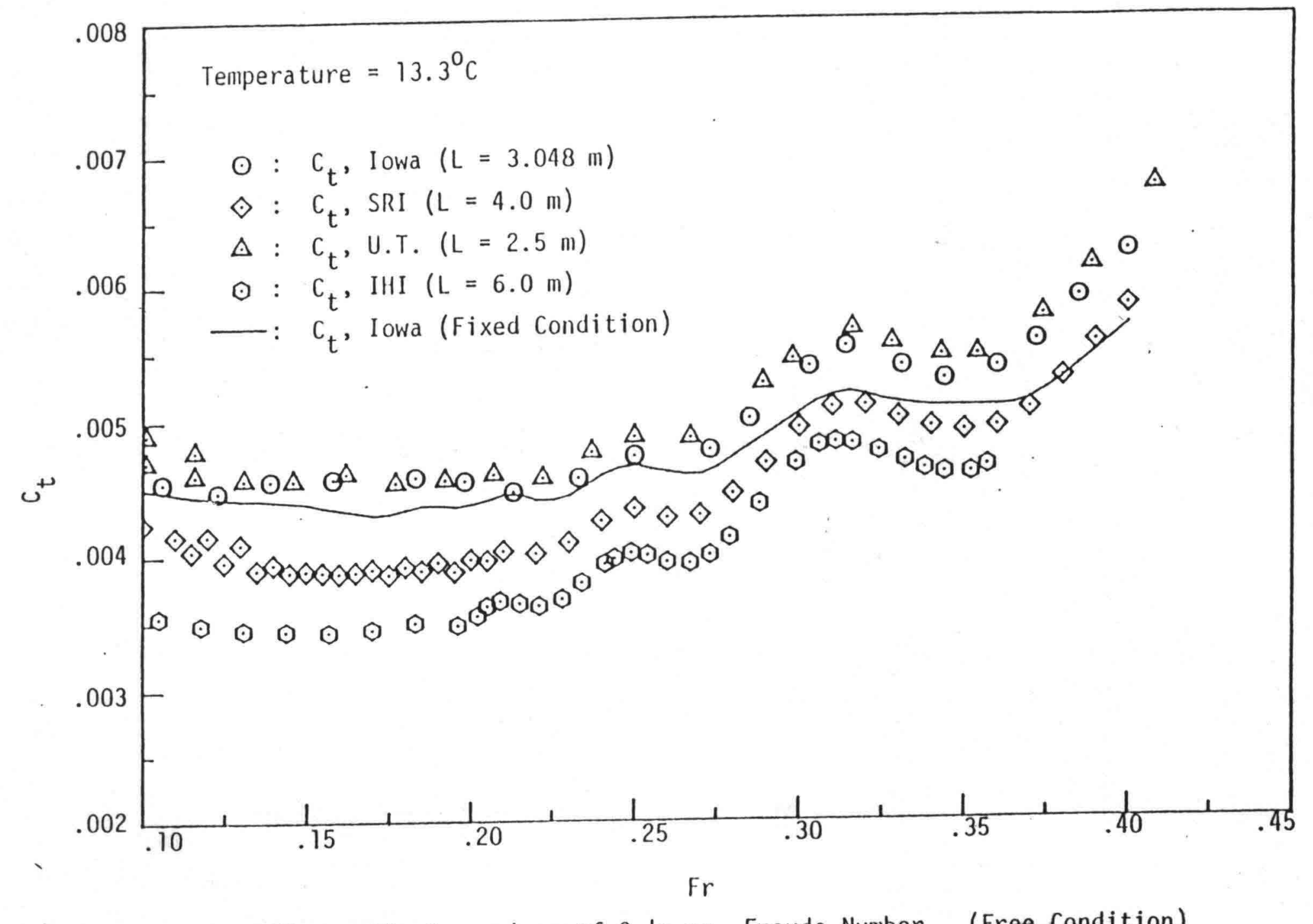

Figure 10. Comparison of $C_{t}{ }^{\prime} s$ vs. Froude Number. (Free Condition) 


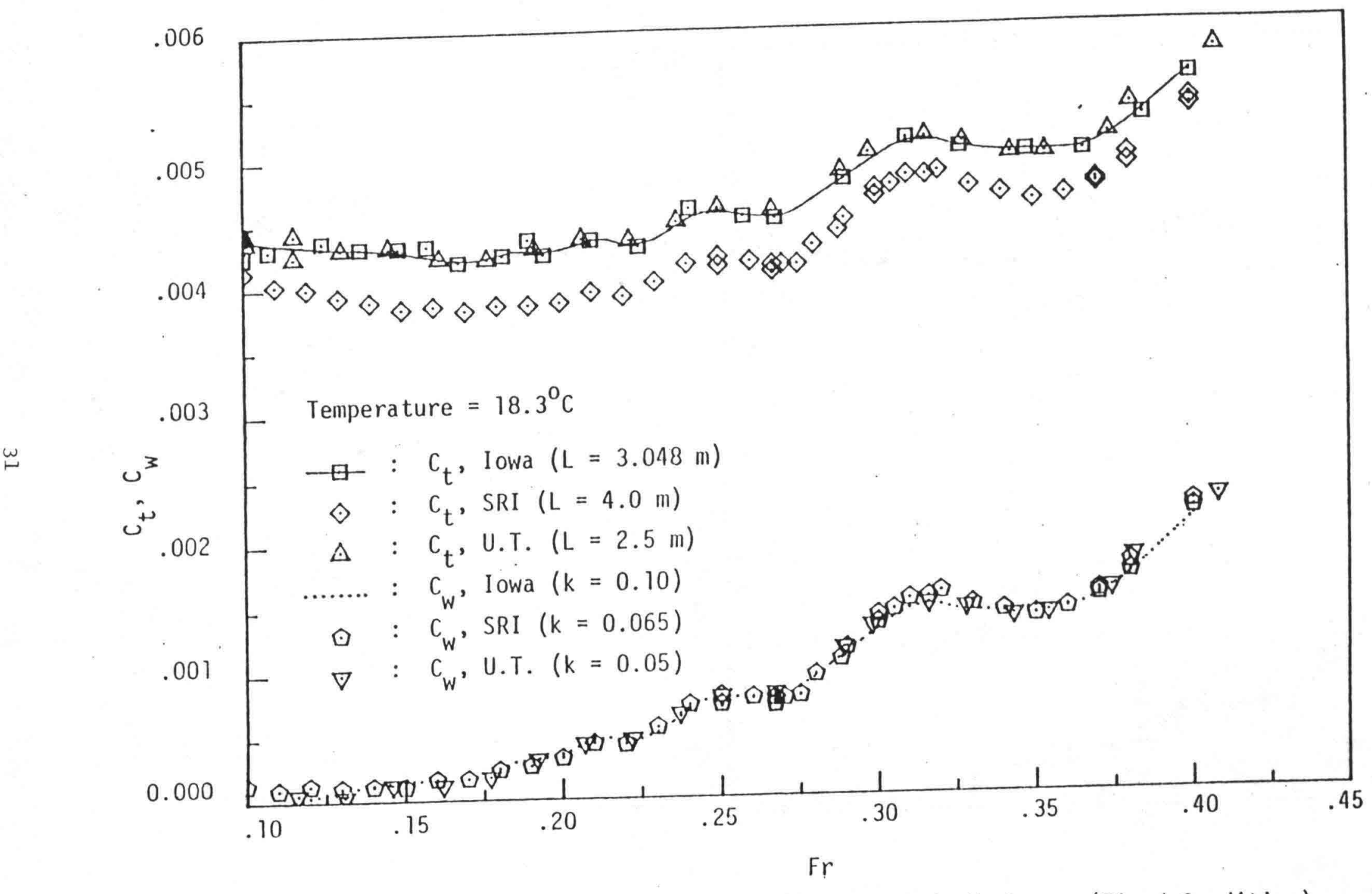

Figure 11. Comparison of $C_{t}{ }^{\prime} s$ and $C_{w}{ }^{\prime} s$ vs. Froude Number. (Fixed Condition) 


\section{Total-Resistance Measurements}

Total-resistance coefficients $C_{t}$, obtained from measurements, are given in Tables $\mathrm{l}$ and 2 from tests at fixed and free-conditions, respectively. These results are compared in Figures 10 and 11 with the other experimental results; those for a 2.5-meter model tested at the University of Tokyo (UT), a 4-m model tested at the Ship Research Intitute of Japan (SRI), and a 6-m model tested at the Ishikawajima-Harima Heavy Industries Co. Ltd. (IHI). The latter model has been tested only in the free condition. To have a meaningful comparison, all the results were corrected to a standard temperature of $18.3^{\circ} \mathrm{C}$ for the fixed-condition tests and $13.3^{\circ} \mathrm{C}$ for the free-condition tests. These results show a similar oscillatory variation with Froude number, i.e. a significant hump and hollow in the range $0.31<\mathrm{Fr}<0.32$ and $0.34<\mathrm{Fr}<0.35$, respectively. There are some additional humps at Froude numbers of 0.21 and 0.25 , and hollows at Froude numbers of 0.22 and 0.27 .

The comparison of results between free and fixed conditions shows that the humps and hollows for both cases occur at the same Froude numbers, but the values of $C_{t}$ with the free condition are greater than those with the fixed condition, by one to four percent for Froude numbers less than 0.28 , and 4 to 10 percent for Froude numbers greater than 0.28 .

The residuary resistance $C_{W}$ for the fixed condition, from the three towing tanks, also have been shown in Figure 10.' These values have been calculated using the form-factor formula

$$
C_{t}=(1+k) C_{f}+C_{w}
$$

where $C_{f}$ is the frictional-resistance coefficient for a flat plate, derived from the Schoenherr (1932) formula, and $k$ is a form factor, which has the values of $k=0.10$ for the Iowa, $k=0.065$ for SRI, and $k=0.05$ for UT. These values were selected so as to obtain the best agreement between the $C_{w}{ }^{\prime} s$ of the three tanks. The higher value for the Iowa towing tank is due to the fact that this tank has the smallest width among the tanks, and as is shown by Landweber and Nakayama (1975), the measured values of total-resistance coefficients are higher than the measured values in a wider tank for $\mathrm{Fr}>0.30$ due to blockage effect of the towing-tank walls. The form-factor procedure gives 
a good estimate of the viscous resistance for the Wigley hull due to its low block coefficient. This has been shown by Ju (1983), who showed that the viscous-resistance coefficient obtained from a wake survey is weakly dependent upon the Froude number for the Wigley hull.

\section{Pressure-Distribution Mesurements}

The results of the pressure-distribution measurements on the Wigley hull along waterlines $|\mathrm{Z} / \mathrm{H}|=0.2$ and 0.6 are shown in Table 3. The pressure coefficient $C_{p}$ is defined as $C_{p}=\left(P_{s}-P_{\infty}\right) / \frac{1}{2} \rho U_{\infty}^{2}$, where $P_{s}$ is the pressure on the hull and $U_{\infty}$ is the speed of the model. The experimental procedure is carried out in such a way that $P_{\infty}=0$ in the above relation. These results are compared with the potential-flow calculations by Guilloton's (1951) method and are shown in Figures 12 through 15. These comparison show fair agreement between calculations and measured values over most of the hull except near the bow and stern. This may be partly due to a logarithmic infinity in the Guilloton calculations at the bow and stern for negative values of $\mathrm{Z} / \mathrm{H}$, i.e. beneath the free surface. At the free surface, the logarithmic part of the calculated functions vanishes, so that the measured wave profile along the hull is in very good agreement with the result of calculations there. An important feature of these figures is the fact that the pressure distribution on the hull closely follows the trend of the wave profile at the free surface, i.e., shows the same hollows and humps. This shows that the Froude number has an important effect on the boundary layer and its development on ship hulls.

\section{Velocity Distribution}

\subsection{General}

A sample of the output-data file for five-hole-probe measurements is shown in Table 4. There are a few points about this output file which should be mentioned in order to have a better grasp of these measurements. The first column shows the distance of measured points from the hull along the $y$-axis, i.e., at constant waterline planes. The first point of these measurements, where the 5-hole probe is in contact with the hull, is denoted as the zerodistance point, and the rest of the points are referenced relative to this point. The actual distance of the probe axis from the hull at this point is $2.15 \mathrm{~mm}$. Columns 5, 6, and 7 show the nondimensional velocity components $u$, 


\section{PRESSURE DISTRIGUTION COLFFICIENTS AI: $2 \mathrm{H}=0.2$}

FROUDE INLKGERS

\begin{tabular}{|c|c|c|c|c|}
\hline $\begin{array}{l}2 X / L \\
== \pm==\end{array}$ & $==2.266$ & $\begin{array}{l}0.313 \\
=====\end{array}$ & $======$ & $\begin{array}{l}0.460 \\
====-\end{array}$ \\
\hline $\begin{array}{l}-0.950 \\
-0.900 \\
-0.850 \\
-0.800 \\
-0.600 \\
-0.400 \\
-0.200 \\
0.000 \\
0.200 \\
0.400 \\
0.600 \\
0.800 \\
0.650 \\
0.900 \\
0.950\end{array}$ & $\begin{array}{r}0.1579 \\
0.1498 \\
0.1498 \\
0.0629 \\
-0.1669 \\
-0.1099 \\
-0.0099 \\
-0.0169 \\
-0.0640 \\
-0.0669 \\
-0.0439 \\
-0.0190 \\
-0.0060 \\
0.0069 \\
0.0399\end{array}$ & $\begin{array}{r}0.1319 \\
0.1319 \\
0.1459 \\
0.1079 \\
-0.0869 \\
-0.1469 \\
-0.0919 \\
-0.0369 \\
-0.0150 \\
-0.0379 \\
-0.0517 \\
-0.0746 \\
-0.0569 \\
-0.0440 \\
-0.0210\end{array}$ & $\begin{array}{r}0.1274 \\
0.1097 \\
0.1006 \\
0.0910 \\
-0.0535 \\
-0.1327 \\
-0.1525 \\
-0.1397 \\
-0.0921 \\
-0.0396 \\
-0.0225 \\
-0.0064 \\
-0.0016 \\
0.0214 \\
0.0337\end{array}$ & $\begin{array}{r}0.1049 \\
0.008 \% \\
0.0517 \\
0.0705 \\
0.0000 \\
0.0512 \\
0.1205 \\
-0.1262 \\
-0.1074 \\
-0.0803 \\
-0.0647 \\
-6.0373 \\
-0.0213 \\
-0.0123 \\
0.0103\end{array}$ \\
\hline
\end{tabular}

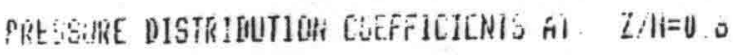

rivoune suHeE

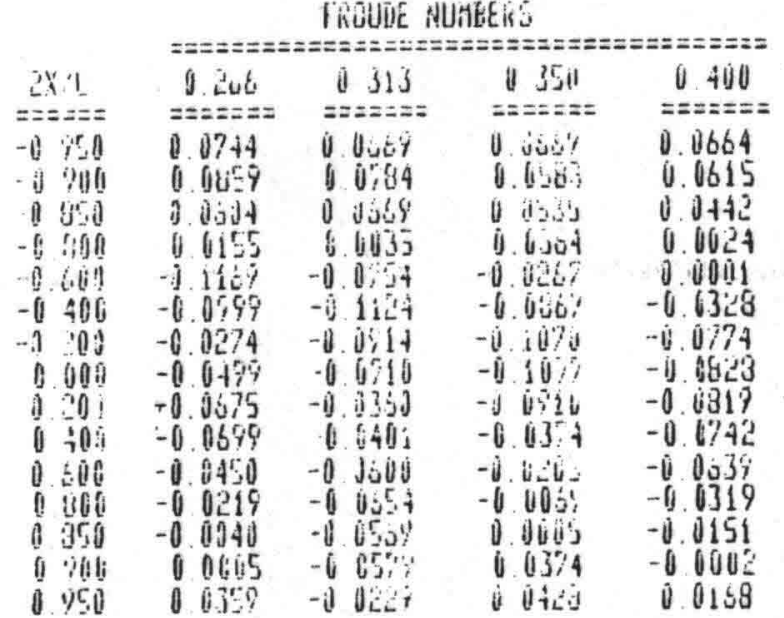

Table 3. Measured pressure-distribution coefficient $C_{p}$. 


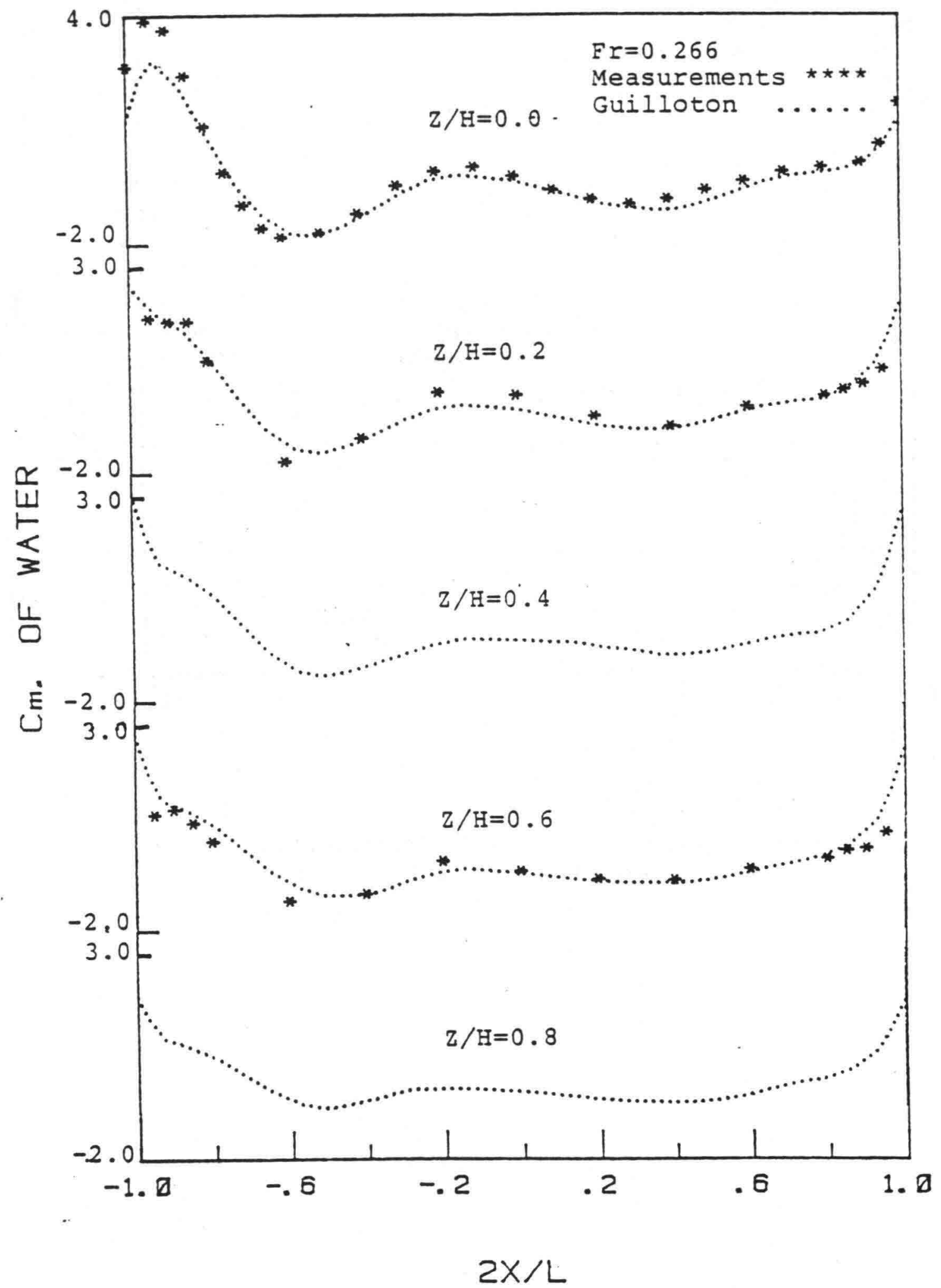

Figure 12. Comparison of measured and calculated pressure distribution $(\mathrm{Fr}=0.266)$. 


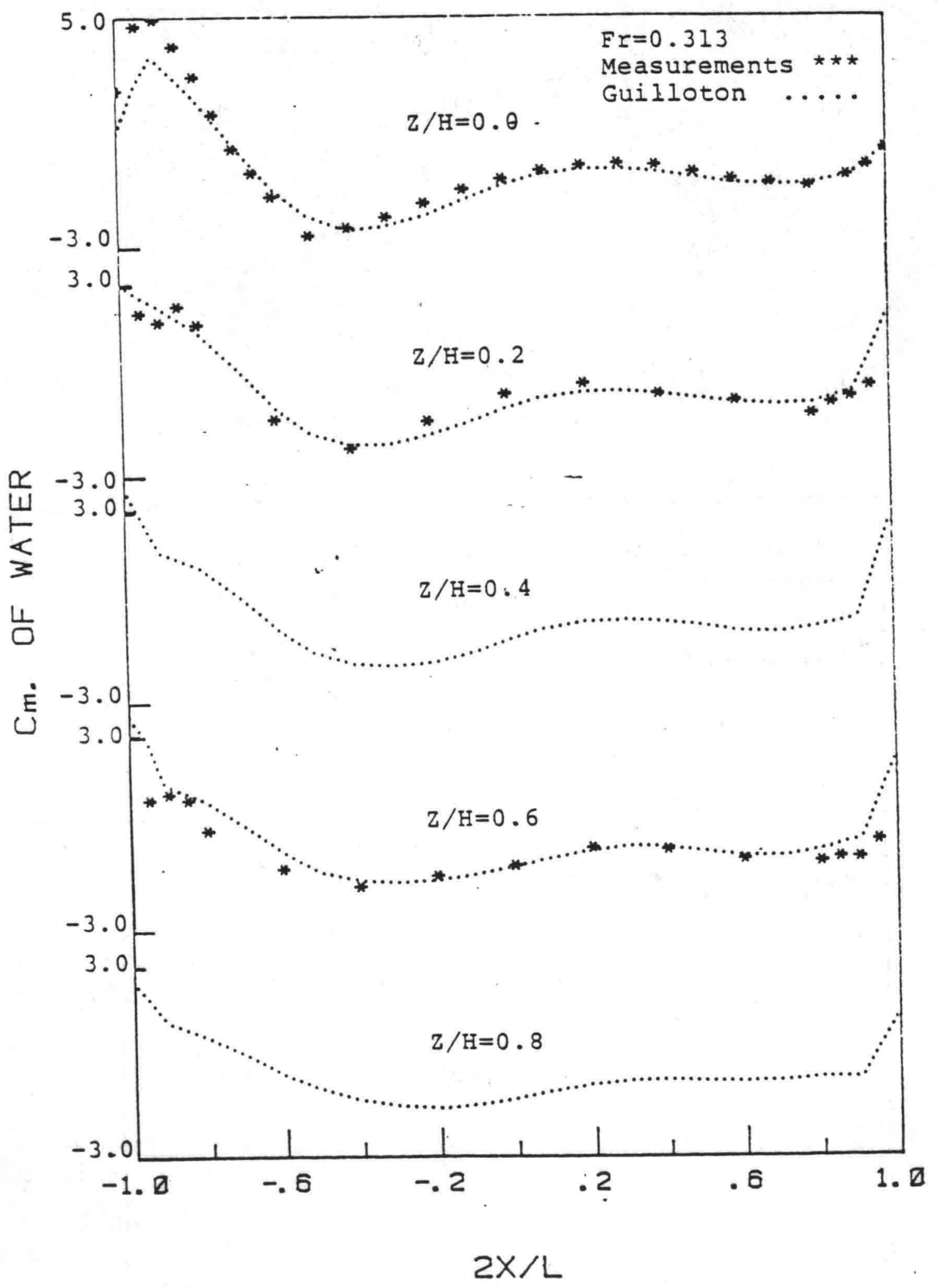

Figure 13. Comparison of measured and calculated pressure distribution ( $\mathrm{Fr}=0.313)$. 


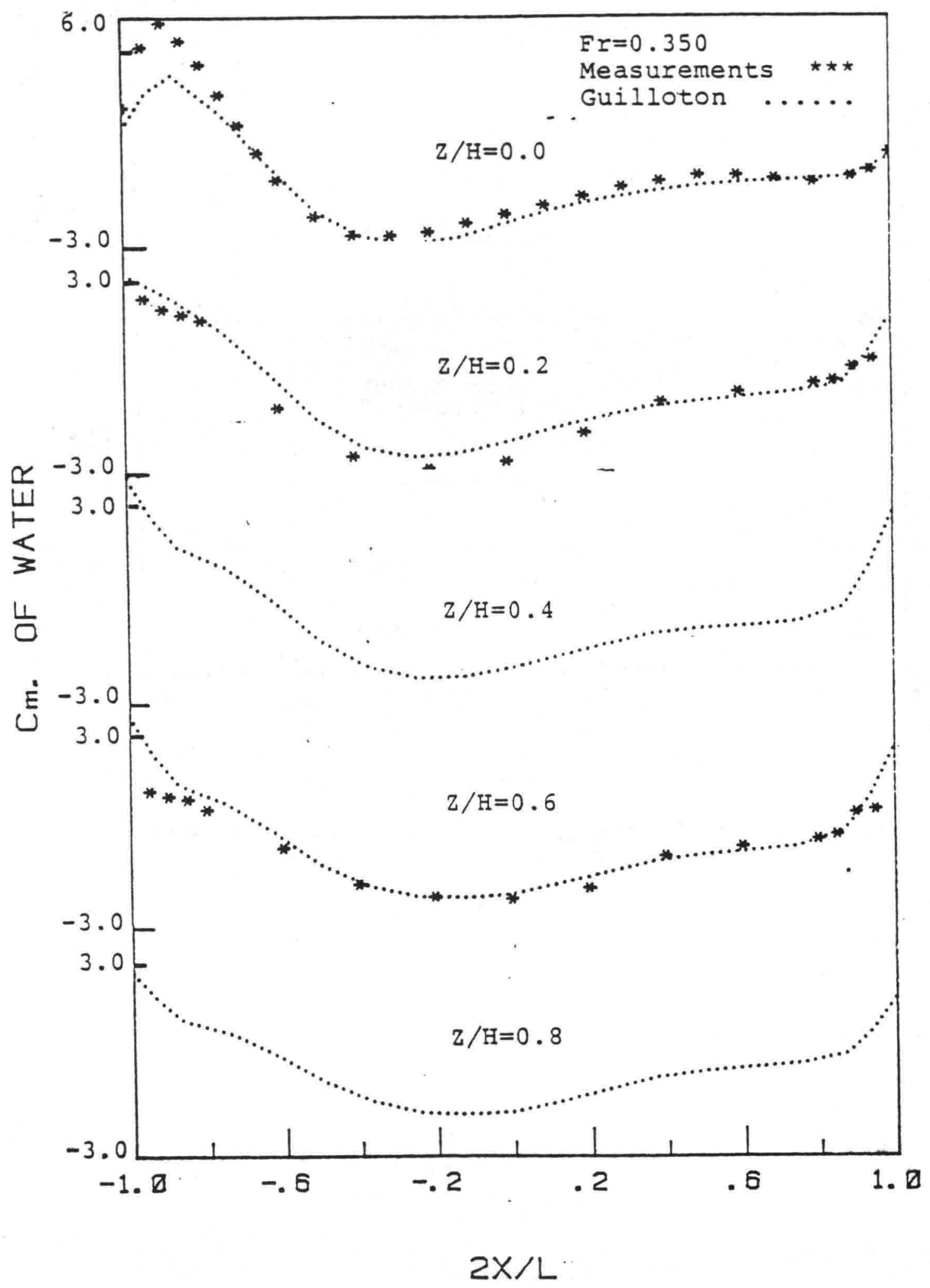

Figure 14. Comparison of measured and calculated pressure distribution $(\mathrm{Fr}=0.350)$. 


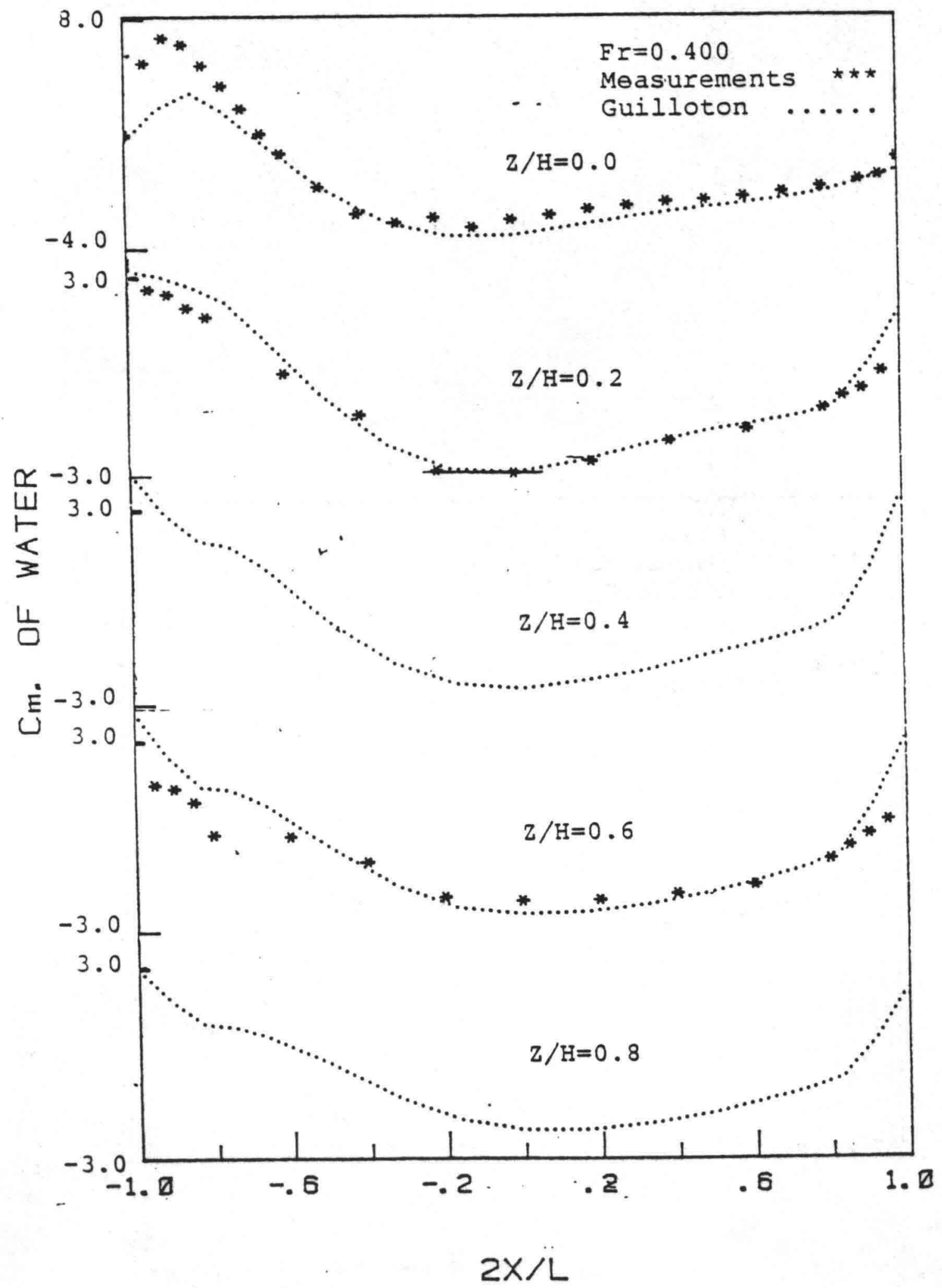

Figure 15. Comparison of measured and calculated pressure distribution $(\mathrm{Fr}=0.400)$. 
BOUNDARY LAYER MEASUREMENTS FOR UIGLEY HULL

\begin{tabular}{|c|c|c|c|c|c|c|c|c|}
\hline \multirow[b]{2}{*}{ DIST (m) } & \multicolumn{3}{|c|}{$\begin{array}{c}\text { LENGTH=304. } 3 \text { CH BEAH } \\
\text { FROUDE NO. }=0.400\end{array}$} & \multicolumn{4}{|c|}{$\begin{array}{l}\mathrm{H}=30.48 \mathrm{CH} \quad \text { DRAFT }=19.05 \mathrm{CH} \\
2 X / L=0.866 \quad Z / H=0.4 \\
=================\end{array}$} & \multirow[b]{2}{*}{ PSI } \\
\hline & $\begin{array}{l}\text { H/H ref } \\
=====\end{array}$ & $==$ & $\begin{array}{l}\text { Q/Q ref } \\
\text { =e=e=s= }\end{array}$ & $\begin{array}{l}\text { U/Q ref } \\
\text { se=e=e= }\end{array}$ & $\begin{array}{l}\text { Y/Q ref } \\
======\end{array}$ & $\begin{array}{l}\text { W/Q ref } \\
======\end{array}$ & $\begin{array}{l}\text { THETA } \\
====\end{array}$ & \\
\hline 0.000 & 0.269 & 0.040 & 0.229 & 0.222 & 0.026 & 0.048 & 6.3 & -12.2 \\
\hline 2.005 & 0.325 & 0.020 & 0.365 & & 0.045 & 0.062 & 8.4 & -11.9 \\
\hline 4.010 & 0.382 & -9.001 & 0.382 & 0.369 & 0.060 & 0.077 & 9.3 & -11.8 \\
\hline 6.015 & 0.421 & -0.005 & 0.427 & 0.413 & 0.065 & 0.085 & 9.1 & -11.7 \\
\hline 8.020 & 0.463 & -0.014 & 0.477 & 0.463 & 0.069 & 0.095 & 8.4 & -11.6 \\
\hline 10.025 & 0.497 & -0.022 & 0.519 & 0.505 & 0.072 & 0.101 & 8.1 & -11.3 \\
\hline 12.030 & 0.529 & -0.02 & 0.552 & 0.507 & 0.072 & 0.107 & 7.7 & -11.2 \\
\hline 14. 030 & 0.557 & -9.026 & 0.583 & 0.508 & 0.074 & 0.111 & 7.5 & -11.0 \\
\hline 16.041 & 0.577 & -1.019 & 0.596 & 0.5 & 0.074 & 0.114 & 7.3 & -11.1 \\
\hline 18.046 & $0.60 \hat{5}$ & -0.020 & 0.625 & 0.589 & 0.078 & 0.117 & 7.3 & -60.9 \\
\hline 20.051 & 0.629 & -9.015 & 0.644 & 0.629 & 0.079 & 0.119 & 7.2 & -10.8 \\
\hline $.22 .05 \dot{0}$ & 0.652 & -0.0 & 0.369 & 0.653 & 0.081 & 0.121 & 7.1 & -10.5 \\
\hline 26.066 & 0.682 & -0.015 & 0.677 & 81 & 0.081 & 0.122 & 6.3 & -10.2 \\
\hline 30.076 & 0.720 & -0.021 & $0.741^{\circ}$ & 0.726 & 0.091 & 0.125 & 6.4 & -9.8 \\
\hline 34.086 & 0.772 & -0.025 & 0.7 & 0.783 & 0.082 & 0.125 & 6.0 & -9.2 \\
\hline 38.098 & 0.827 & -0.024 & 0.852 & 0.938 & 0.085 & 0.126 & 5.8. & -9.6 \\
\hline 42.107 & 0.875 & -0.022 & 0.897 & 0.884 & 0.088 & 0.125 & 5.7 & -8.1 \\
\hline 46.117 & 0.919 & -0.018 & 0.737 & 0.925 & 0.089 & 0.123 & 5.5 & -7.6 \\
\hline 50.127 & 0.944 & & 0.960 & 0.950 & 0.090 & 0.121 & & \\
\hline 68.152 & 0.986 & -0.010 & 0.996 & 0.989 & 0.088 & 0.114 & 5.0 & -6.6 \\
\hline 70.178 & 1.005 & -0.008 & 1.0 & 1.008 & 0.082 & 0.109 & 4.7 & -6 \\
\hline $80.20 Z$ & 1.012 & -0.007 & 1.019 & 1.011 & 0.077 & 0.104 & 4.4 & \\
\hline 90.283 & 1.011 & -3.008 & 1.019 & 1.011 & 0.074 & 0.099 & 4.1 & -5 . \\
\hline 110.279 & 1.003 & -0.011 & 1.014 & 1.008 & 0.071 & 0.091 & 3.9 & -5. \\
\hline 139.330 & 0.999 & -8.015 & 1.014 & 1.009 & 0.967 & 0.094 & 3.5 & \\
\hline
\end{tabular}

Table 4. Sample of output file on tape. 
$\mathrm{v}$, and $\mathrm{w}$ along the $\mathrm{x}, \mathrm{y}$, and $\mathrm{z}$ axes, respectively. $\mathrm{u}>\mathrm{o}$ denotes the velocity direction towards the stern; $w>0$, represents the velocity direction from keel toward free surface; and $v>0$ denotes the fllow direction towara tne hull. Column 4 shows the nondimensional magnitude of the velocity vector, and column 3 gives the pressure coefficients measured by the five-hole probe. Column 2 represents the total head which is obtained from columns 3 and 4 . Columns 8 and 9 show the pitch and yaw angles, respectively, which are defined in Figure 2. The complete set of data for all four Froude numbers, is archived on magnetic tape and is available from the IIHR tape library.

\subsection{Velocity Distribution on the $\mathrm{Hull}$}

Figures 16 through 20 show typical distributions of the u-component of the mean velocity vector. It is seen that the flow is of a mild adversepressure-gradient type. The profiles also seem to have a characteristic concavity near the wall. This is due to the beginning of the wake-like behavior of the outer (fully turbulent) layer. These figures show that the boundary layer is rather thin over most of the hull, except near the stern where the thickness becomes of the order of four percent of the model length $\left(\frac{\delta}{\mathrm{L}}=0.04\right)$; but still it may be regarded as a 'thin' boundary layer. There are no drastic changes in the profile in the $\mathrm{x}$-direction. Also, there is no reversal of the u-component velocity even at the stern, which indicates that the flow does not separate over the Wigley hull. An interesting feature of the boundary layer which can be seen from these figures is its rate of growth. The boundary-layer thickness at the waterline $|\mathrm{Z} / \mathrm{H}|=0.2$ grows along the hull; see Figure 16; however, the rate of growth decreases at the deeper waterlines, as is seen at $|\mathrm{Z} / \mathrm{H}|=0.4$ (Figure 17) and becomes almost constant at $|\mathrm{Z} / \mathrm{H}|=0.6$; see Figure 18. Near the keel, the rate of growth reverses, as is shown in Figures 19 and 20 for $|\mathrm{Z} / \mathrm{H}|=0.8$ and $|\mathrm{Z} / \mathrm{H}|=1.0$, respectively. There the boundary-layer thickness is largest amidships and diminishes along the hull until the stern where it becomes almost zero. This phenomenon can be attributed mainly to the geometry of the Wigley hull. The streamlines from bow to midship converge toward the keel, causing the boundary layer to become thick near the keel at midship. From there to the stern, the reverse of the aforementioned phenomenon occurs; i.e. the streamlines diverge from the keel, causing the boundary layer to become thinner as the flow approaches the stern. 
Consider now the distribution of the $\mathrm{v}$ - and w-components of the mean velocity. The w-component at all the sections is positive, i.e., its direction is from the keel towards the free surface. This can be explained by noting that the wave profiles on the hull have a trough at about the midship. As will be shown in Chapter IV, the streamlines on the hull closely follow the trend of the surface-wave profiles, so that the flow from midship to the stern is also upward; i.e., the $w$ component will be positive in that region.

The direction of the v-component of the velocity, for the most part, is toward the hull. This should be expected since the flow from midship, where the hull is thickest, converges on the both sides of the model toward the stern. This implies that the velocity vector has a component in the negative direction of the $y$-axis on the starboard side where the measurements were taken. Figures 21 through 28 show some typical vector plots of the v-w components. An interesting feature of these plots is shown at the $|\mathrm{Z} / \mathrm{H}|=0.2$ waterlines which are very close to the free surface. Since $\frac{w}{v}=\frac{d z}{d y}$ at the free surface, the slope of the velocity vector at this waterline indicates the effect of the boundary layer on the lateral slope of the free surface.

\section{Wake Measurements}

The mean-velocity components were measured at four stations from the stern to $30.48 \mathrm{~cm}$. Figures 29 through 32 show the typical distribution of the $\mathrm{u}$ components of the velocity vector. The profiles are seen to be symmetric. The point of the minimum velocity occurs at the wake centerline. Since the measurements were not carried out far enough, it was not possible to investigate the decay of three dimensionality, or the asymptotic behavior of the wake. Figures 33 through 40 show the distribution of the $v-w$ components at $\frac{2 \mathrm{x}}{\mathrm{L}}=0$ and $\frac{2 \mathrm{x}}{\mathrm{L}}=1.2$, for various Froude numbers.

\section{Effects of Froude Number on Boundary Layer}

The practical importance of free-surface phenomena in viscous flow around ships has been reviewed by Baba (1981). Also Landweber (1971), and Landweber and Patel (1979) have discussed in detail the various effects of the free surface, such as the free-surface boundary layer, bilge vortices, breaking of the bow wave and separation of free surface. In this section, we shall point 
out the variation of the measurement data caused by changes of the Froude number, and also compare the present results with the measurements obtained by Sarda (1985) on a double model of the Wigley hull of the same dimensions, in the IIHR wind tunnel.

The first characteristic showing the influence of the Froude number is the pressure distribution over the hull. In the case of zero Froude number, the measured pressure coefficient is a nearly-symmetric curve about the midship at any waterline plane. In the case of nonzero Froude numbers, however, the measured pressure coefficients are strongly affected by the wave profile along the hull, and closely follow the trend of the free surface as is shown in Figures 12 through 15. This fact is re-emphasized in Figures 41 through 44. These figures show the comparison of the measured pressure coefficient and wave profile with $1-\left(\frac{e}{Q_{e f}}\right)^{2}$, where $Q_{e}$ is the magnitude of the velocity vector at the edge of the boundary layer. This consequence of the constant free-surface pressure at nonzero Froude numbers significantly affects many characteristics of the boundary layer, such as the rate of growth, the magnitude of the cross flow, the piezometric pressure gradients, and the velocity at the edge of the boundary layer. Figure 45 shows the comparison of the displacement thickness along the hull at $|\mathrm{z} / \mathrm{H}|=0.2$ for different Froude numbers. There is a combination of Reynolds-and-Froude-number effects on the rate of growth of the boundary layer along the hull. The Reynolds number influences the boundary layer through viscosity whereas the Froude number imposes itself mainly through pressure distribution. At the first station, $2 \mathrm{x} / \mathrm{L}=0.0$, the ratio of displacement thickness at $\mathrm{Fr}=0.266$ to the one at $\mathrm{Fr}$ $=0.400$ is about 2. This ratio for comparable Reynolds numbers of a double model is about 1.2. This is the first indication of Froude-number effect on boundary-layer development. The rate of growth of boundary layer for different Froude numbers then exactly follows the trend of the pressure distribution at $\mathrm{z} / \mathrm{H}=0.2$ (Figures 12 through 15, or Table 3). From midship to $2 \mathrm{x} / \mathrm{L}=0.4$, the flow is decelerating for all the Froude numbers except $\mathrm{Fr}=$ 0.266. Therefore the displacement thickness has the smallest rate of growth for $\mathrm{Fr}=0.266$ in that region. From $2 \mathrm{x} / \mathrm{L}=0.4$ to $2 \mathrm{x} / \mathrm{L}-0.6$, displacement thickness has its largest value for $\mathrm{Fr}=0.350$. This is because flow over the Wigley hull at this station has the greater rate of deceleration in comparison with the other Froude numbers, and therefore the boundary layer becomes thick faster. Between $2 x / L=0.6$ and $2 x / L=0.8$ stations, only flow at $\mathrm{Fr}=0.313$ 
is accelerating and the displacement thickness at this Froude number has a smaller value in comparsion with the other Froude numbers. Finally, near the stern, the displacement thickness at $\mathrm{Fr}=0.266$ has the largest rate of growth because it has the largest unfavorable pressure gradient and flow decelerates at a faster rate than at the other Froude numbers. Figures 25 through 28 show the vector plot of crossflow at $2 \mathrm{x} / \mathrm{L}=0.866$. The wave profile on the hull at this station has slopes of $0.051,0.034,0.032$, and 0.046 for Froude numbers of $0.266,0.313,0.350$, and 0.400, respectively. Comparison of these figures shows that the magnitude of the crossflow is directly related to the slope of the free surface. For Froude numbers of 0.313 and 0.350 , where the slope is almost the same, the crossflow vectors are very similar and their magnitudes are smaller than those at Froude numbers of 0.266 and 0.400 . As a matter of fact, at $\mathrm{Fr}=0.266$, the crossflow is larger than at the other Froude numbers. Figures 46 through 49 show the comparison of the yaw angle $\Psi$, which is directly proportional to the magnitude of the crossflow component of the velocity vector, at two stations $2 x / L=0.8$ and 0.933 , for zero and nonzero Froude numbers. The comparison shows that the yaw angles for nonzero Froude numbers are at least 200 percent of the comparable angles for the double model. This comparison also show that the effect of Froude number on the boundary layer is felt primarily near the free surface, as is shown in Figures 46 and 48 for $|z / H|=0.2$. Figures 47 and 49 show the yaw-angle distribution across the boundary layer for $|\mathrm{z} / \mathrm{H}|=0.8$. Note that the yaw angles for different Froude numbers almost collapse into a single curve near the keel, but still they are almost twice the comparable angles for the double model. The effect of the free surface on cross-flow extends into the wake region of the model. Figures 37 through 40 show the cross-flow plots at $2 x / L=1.2$ for different Froude numbers. Note the distinct S-shaped profile which occurs at Froude numbers 0.266, 0.313, and 0.350, while, at $\mathrm{Fr}=0.400$ the cross-flow does not show such a behavior. 


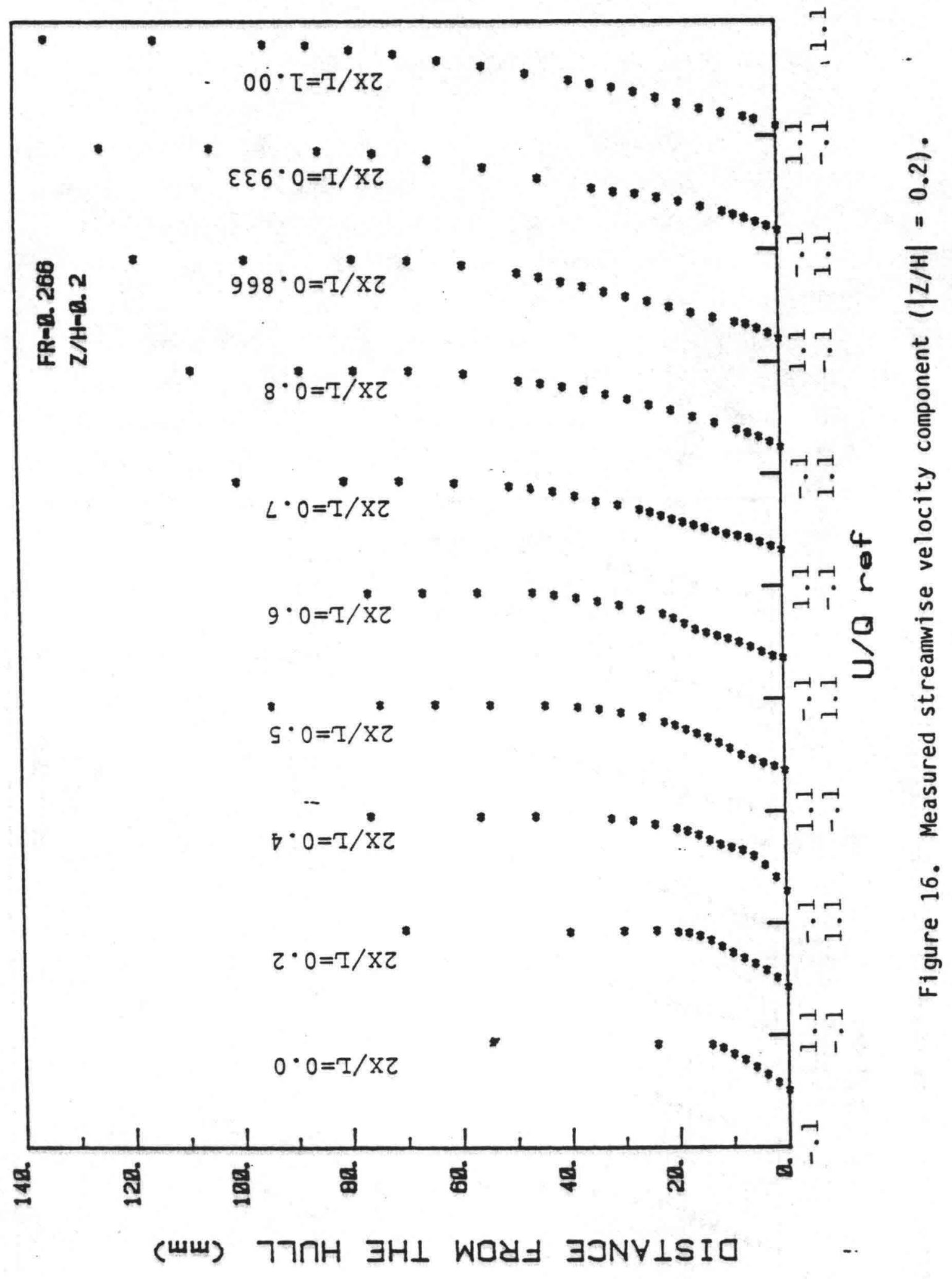




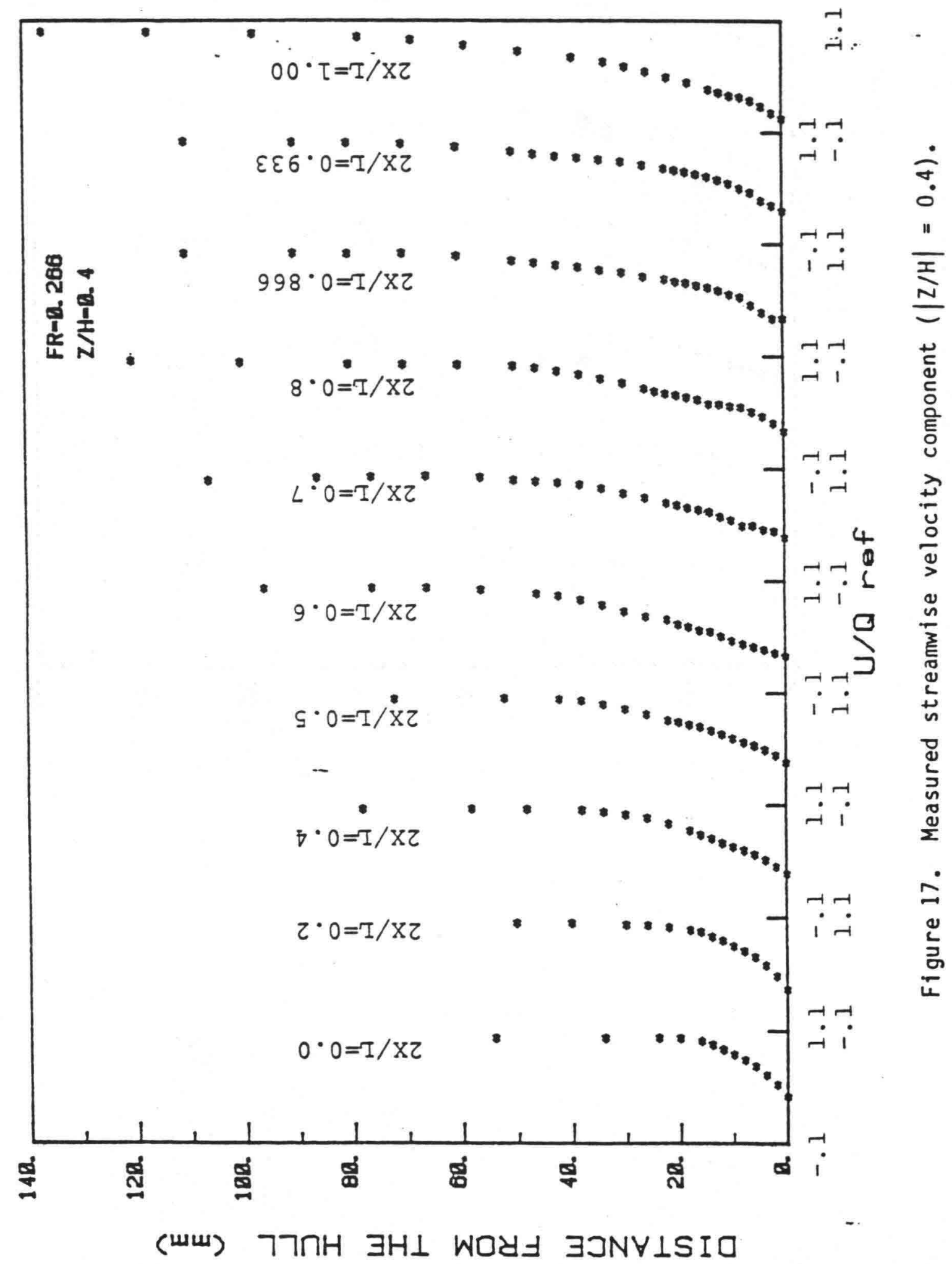




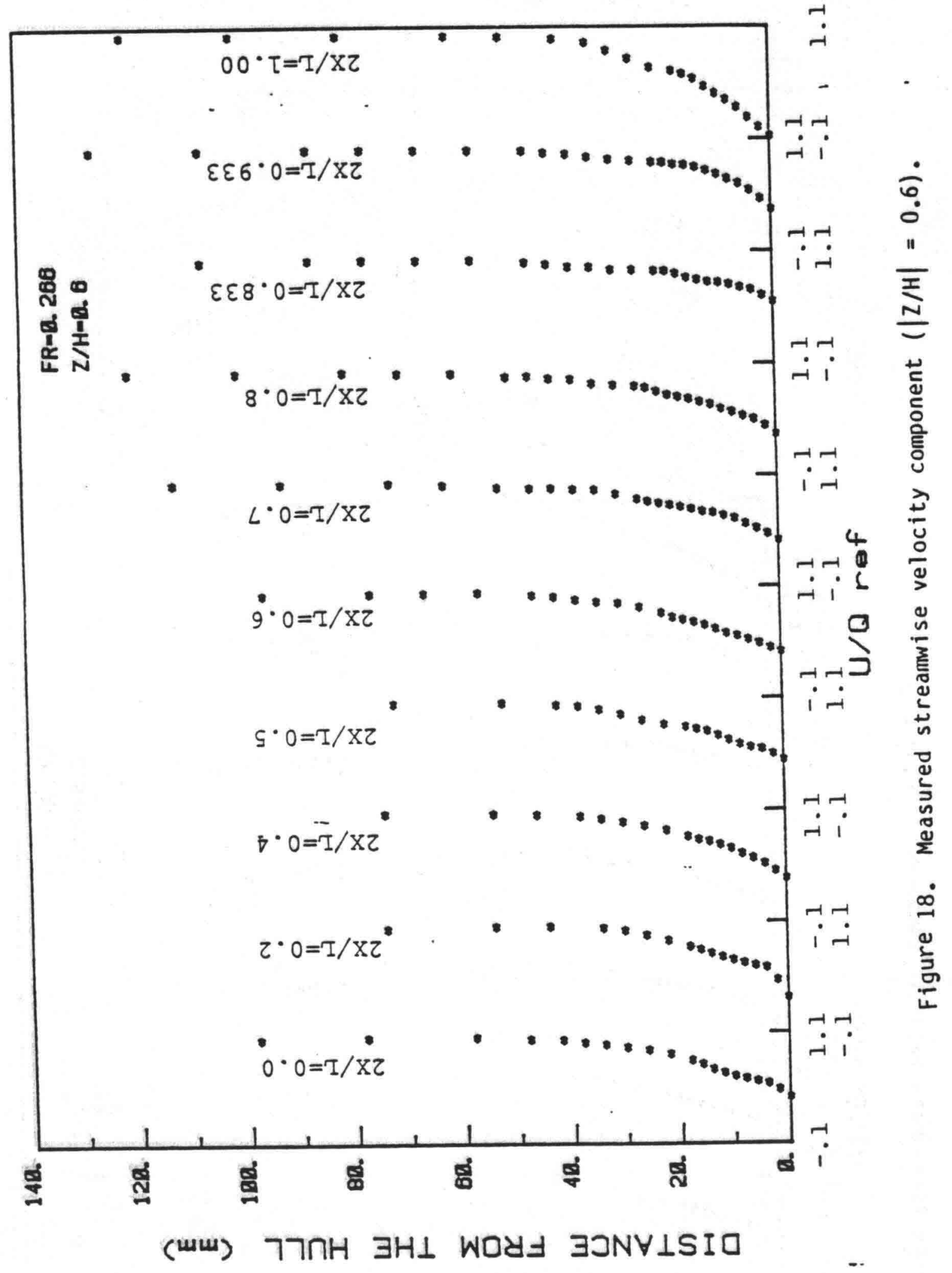




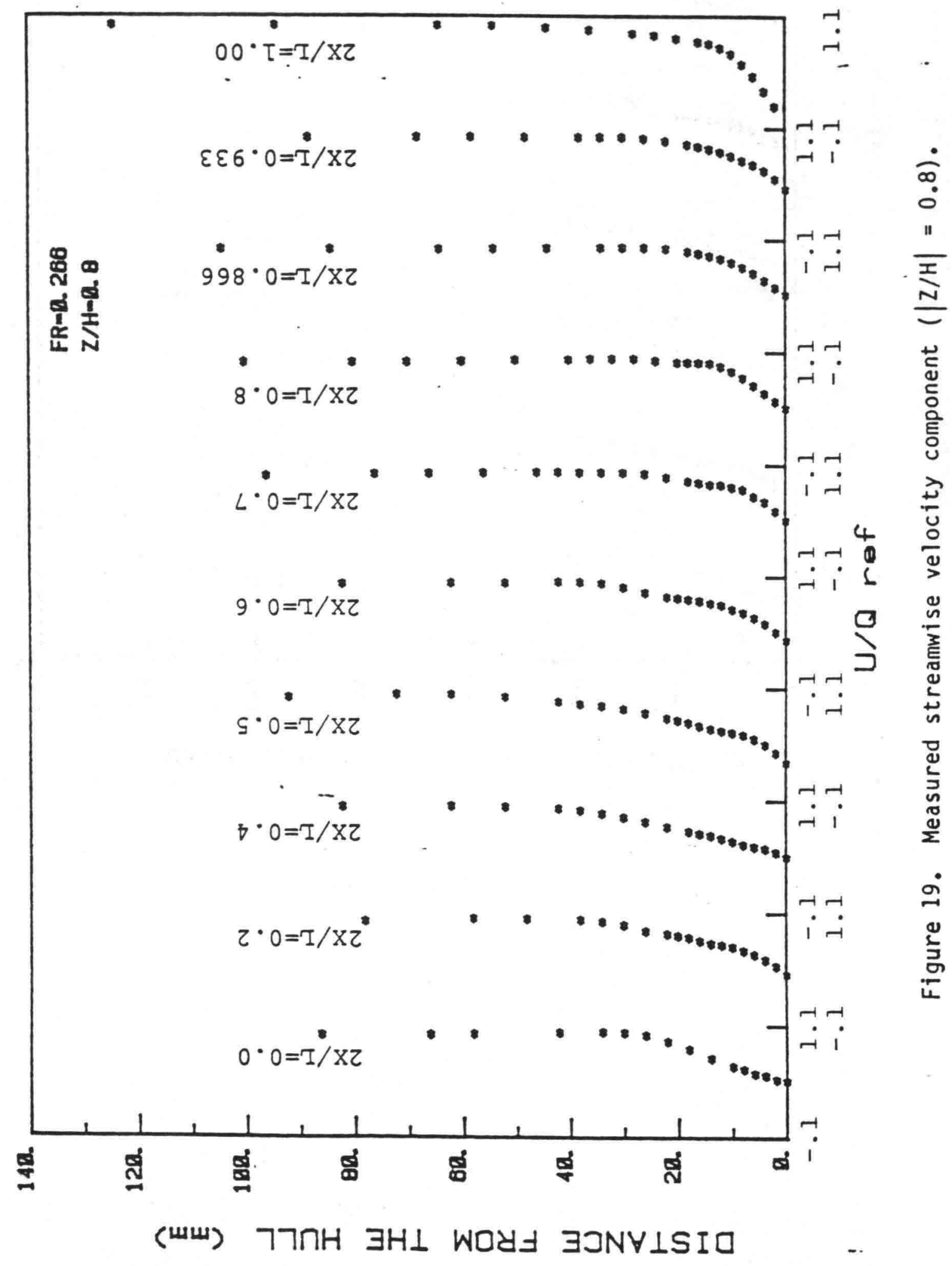




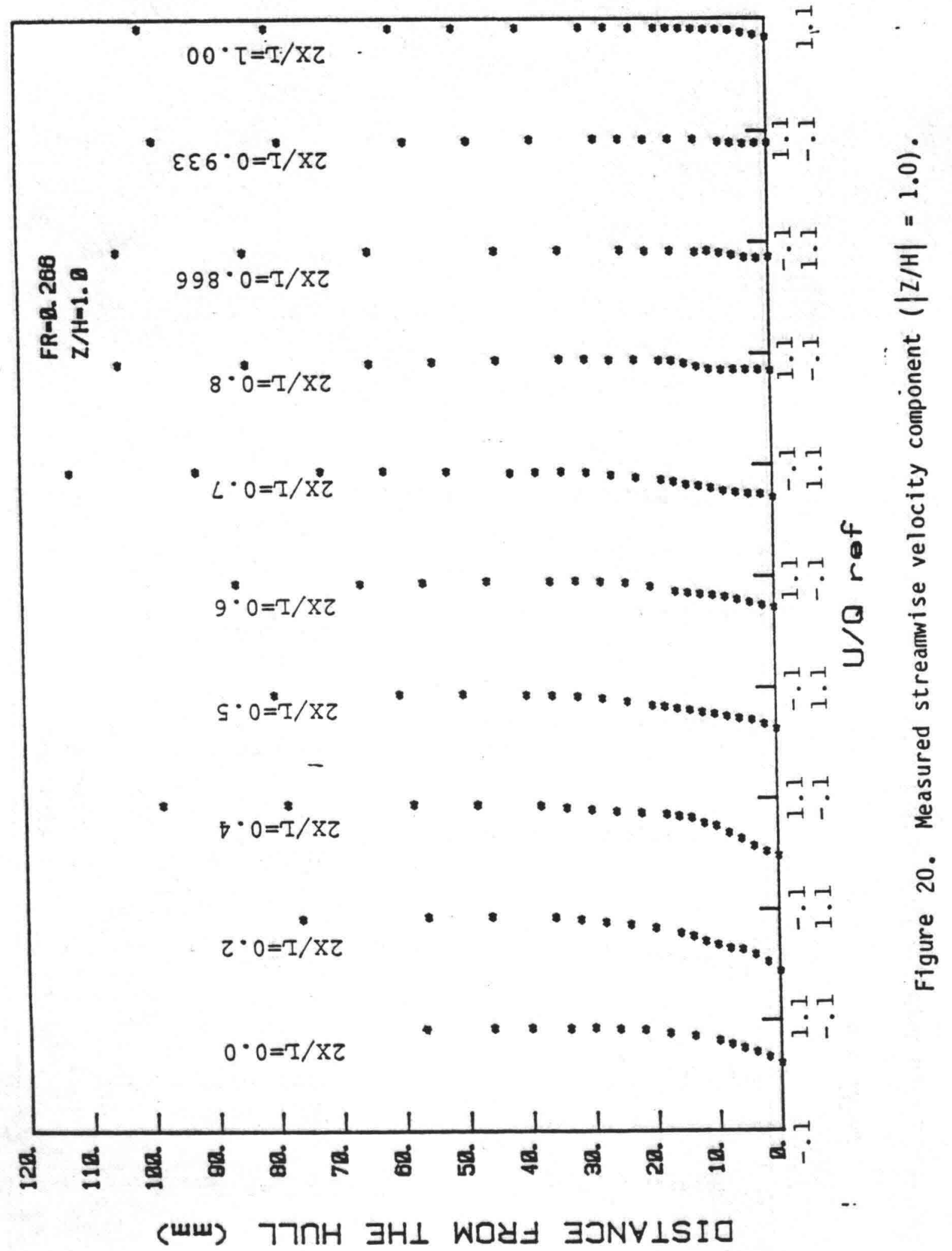




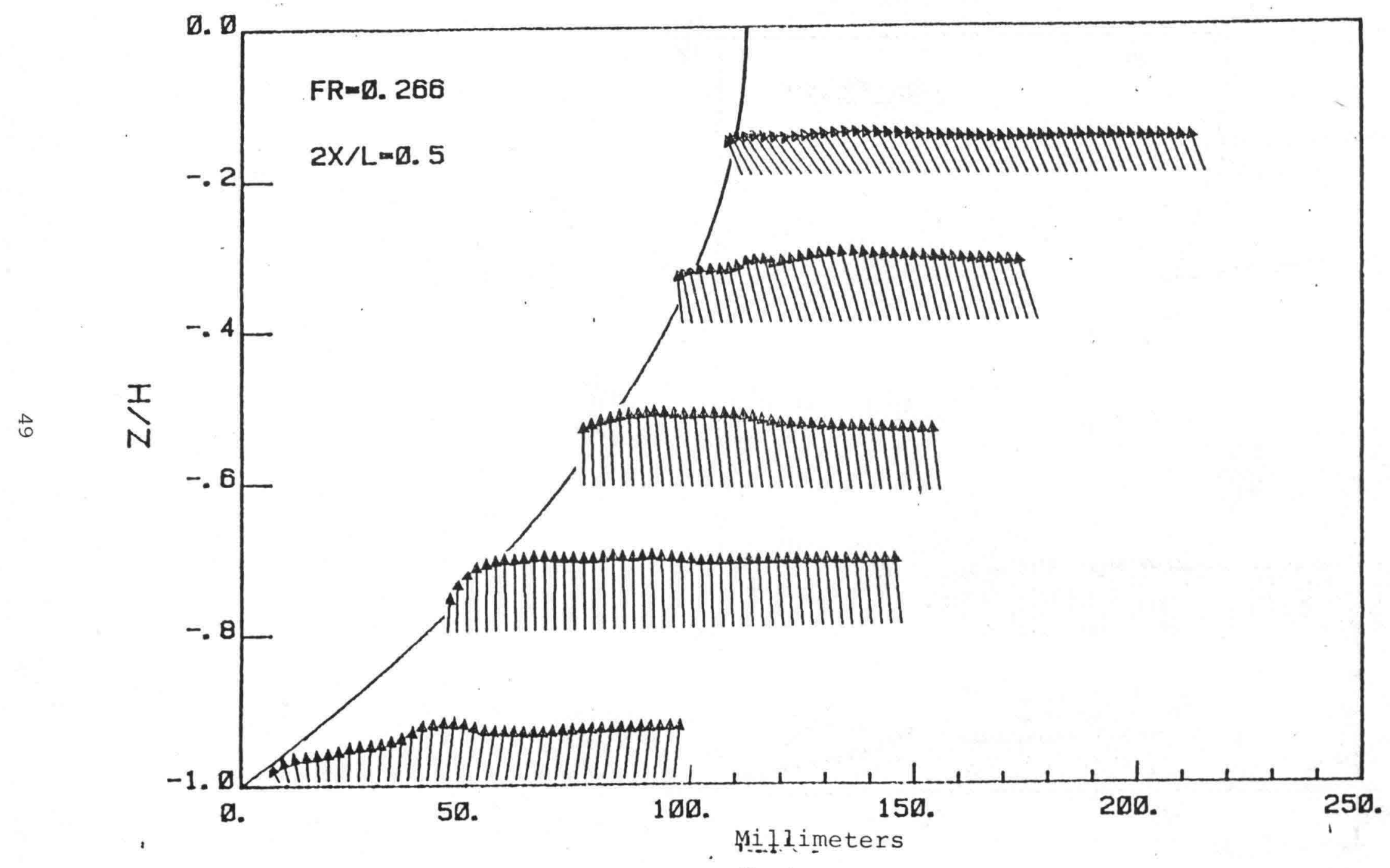

Figure 21. Vector plot of $w-v$ components $(2 x / L=0.5, F r=0.266)$. 


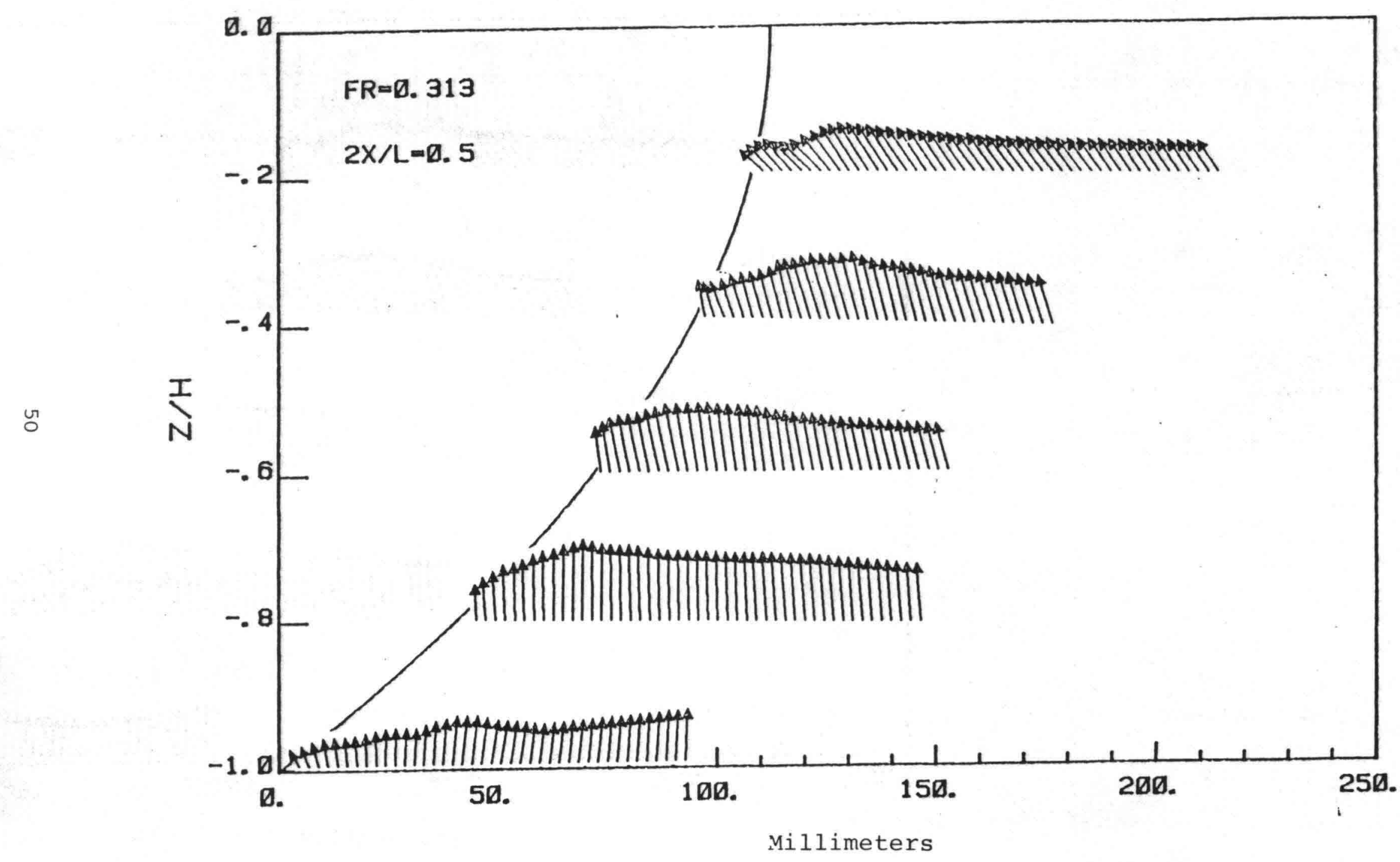

Figure 22. Vector plot of $W-v$ components $(2 x / L=0.5, F r=0.313)$. 


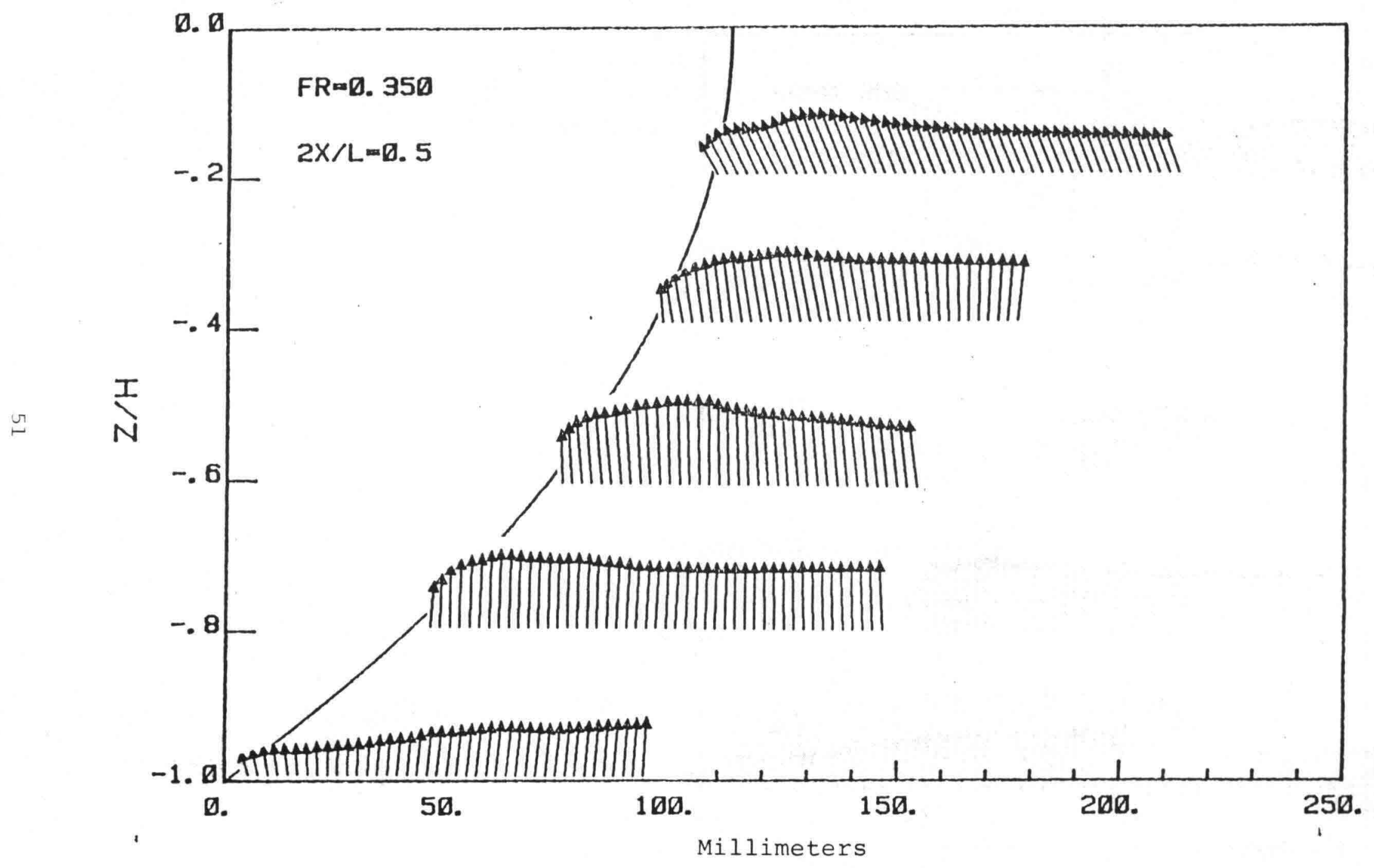

Figure 23. Vector plot of $w-v$ components $(2 x / L=0.5, F r=0.350)$. 


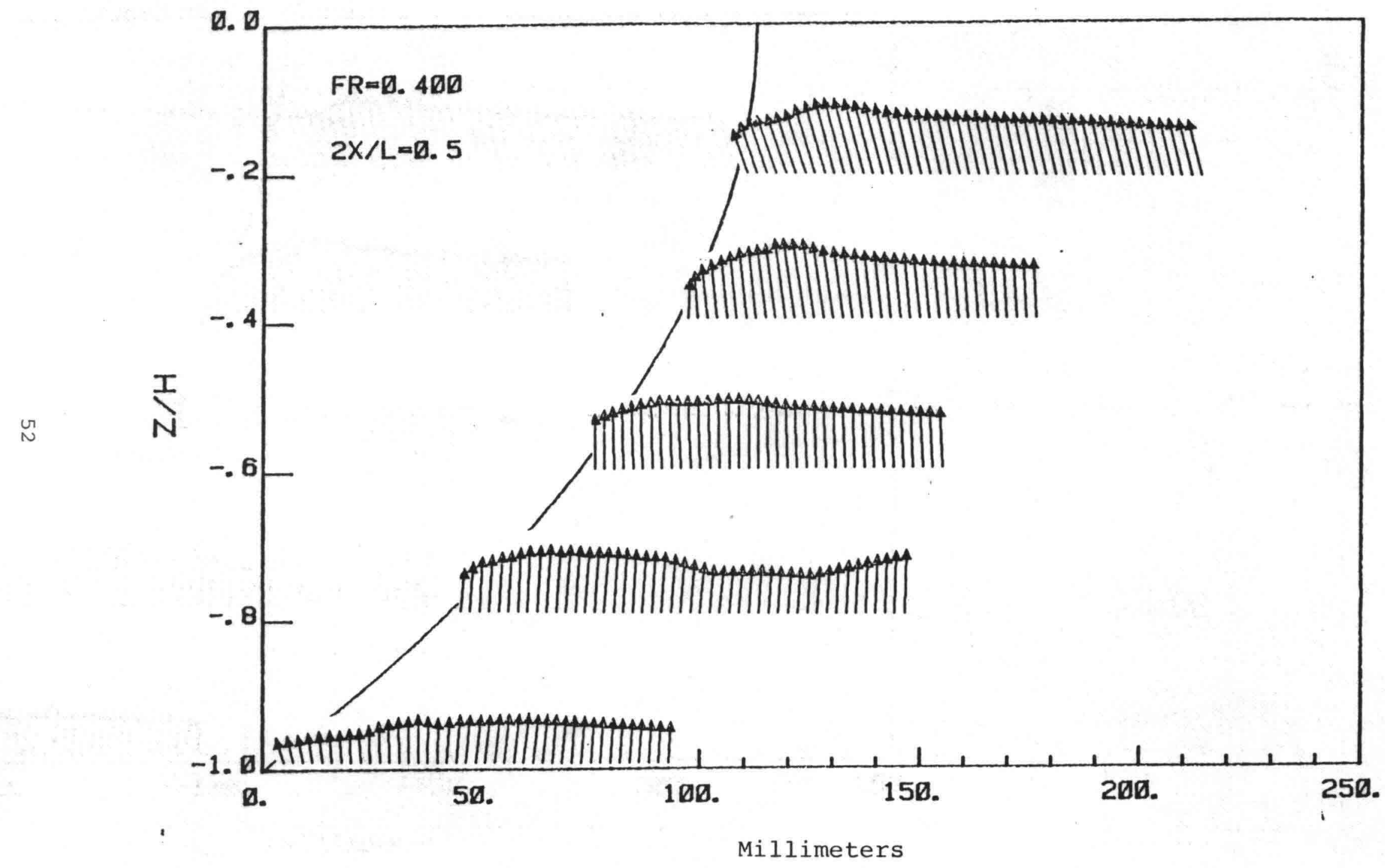

Figure 24. Vector plot of $w-v$ components $(2 x / L=0.5, F r=0.400)$. 


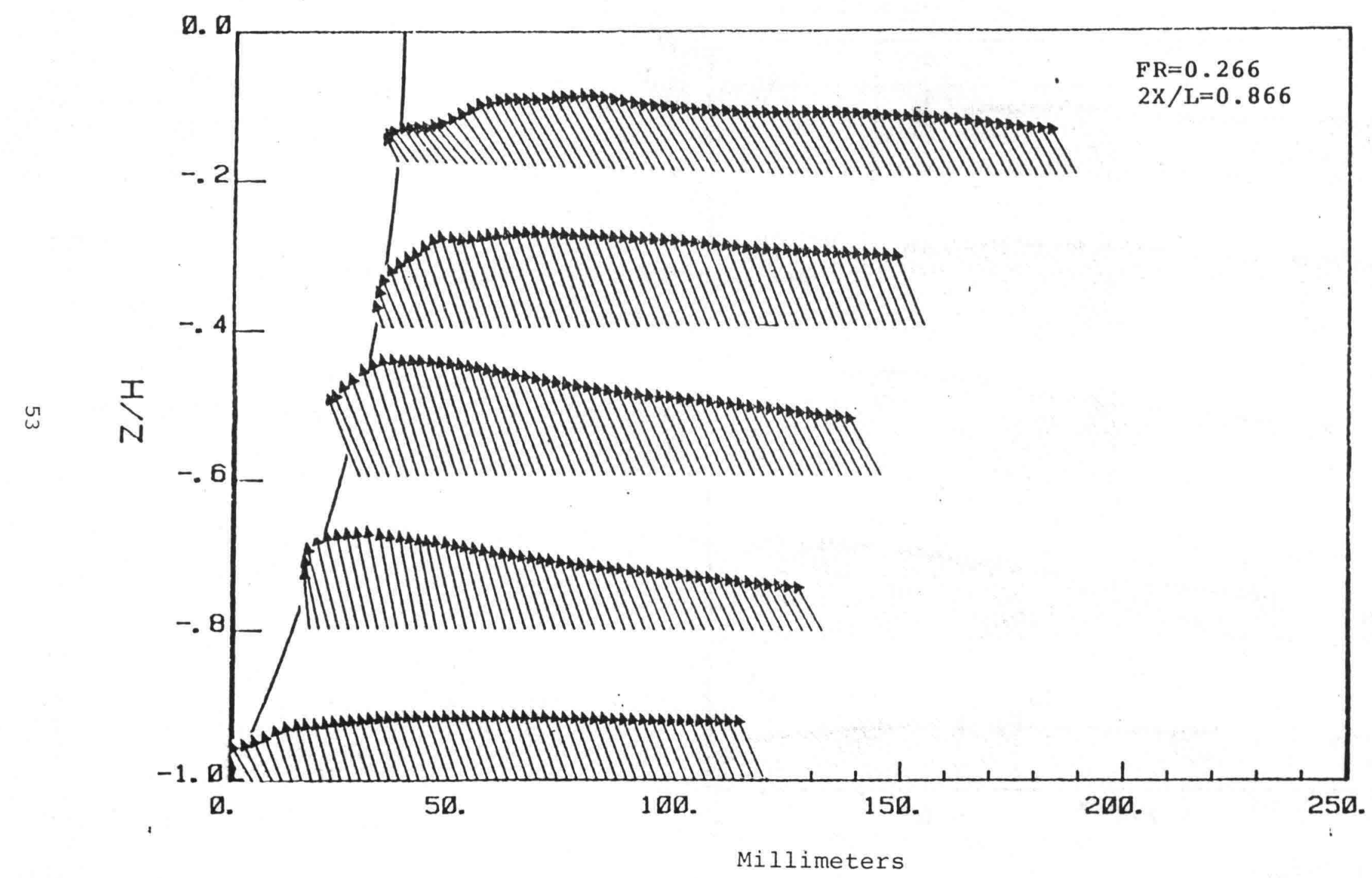

Figure 25. Vector plot of $w-v$ components $(2 x / L=0.86, F r=0.266)$. 


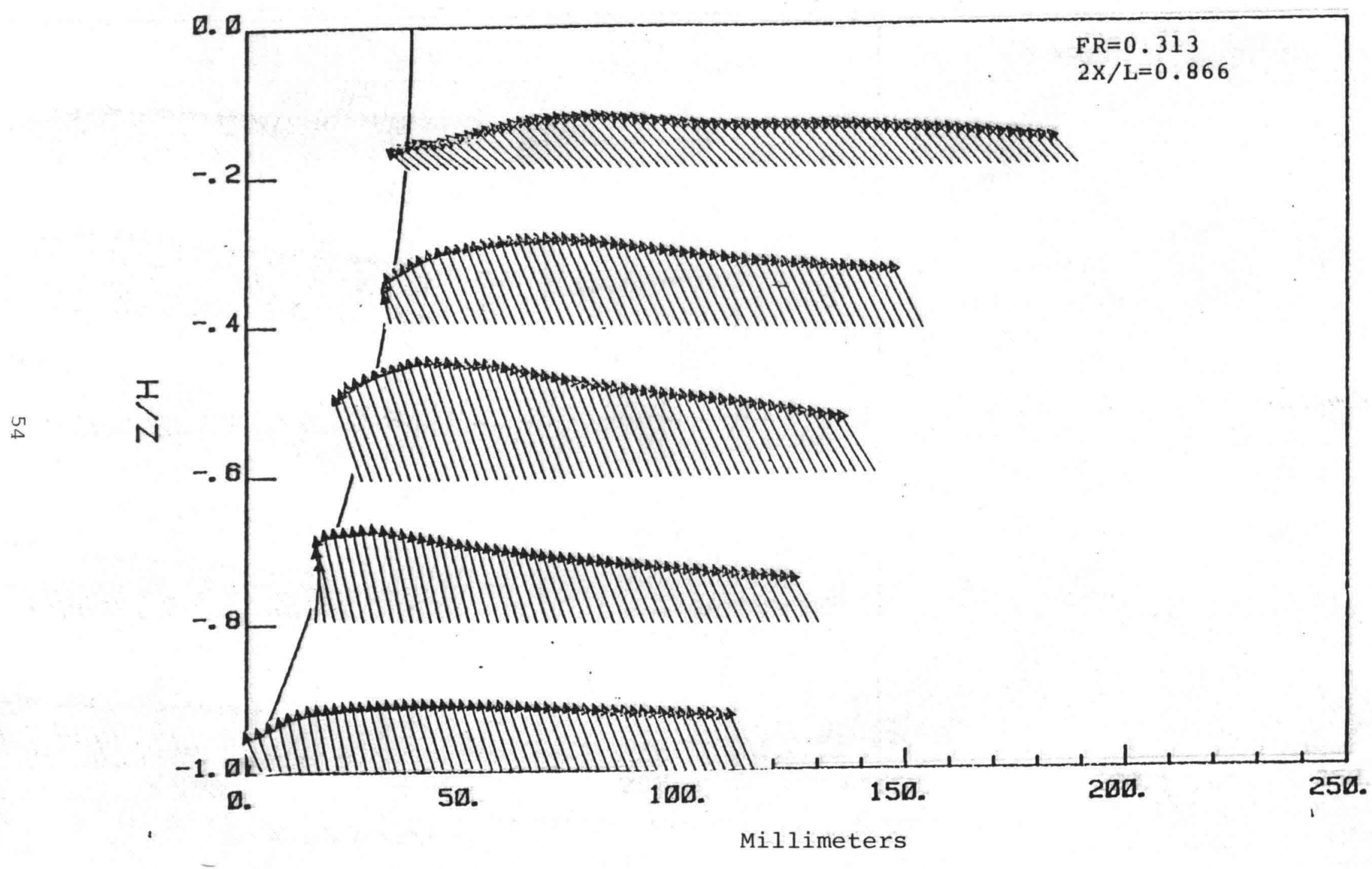

Figure 26. Vector plot of $w-v$ components $(2 x / L=0.86, F r=0.313)$. 


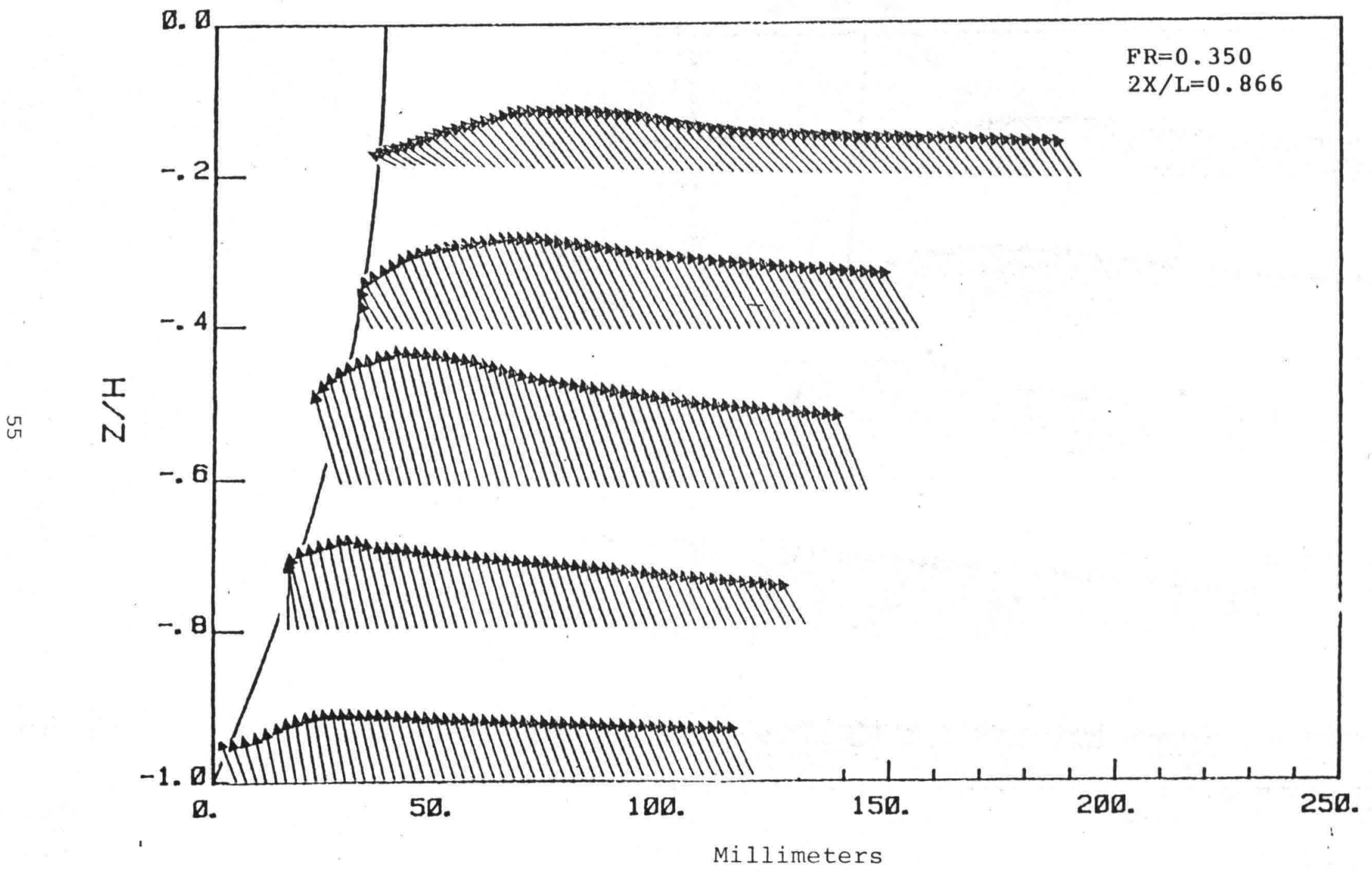

Figure 27. Vector plot of $w-v$ components $(2 x / L=0.86, F r=0.350)$. 


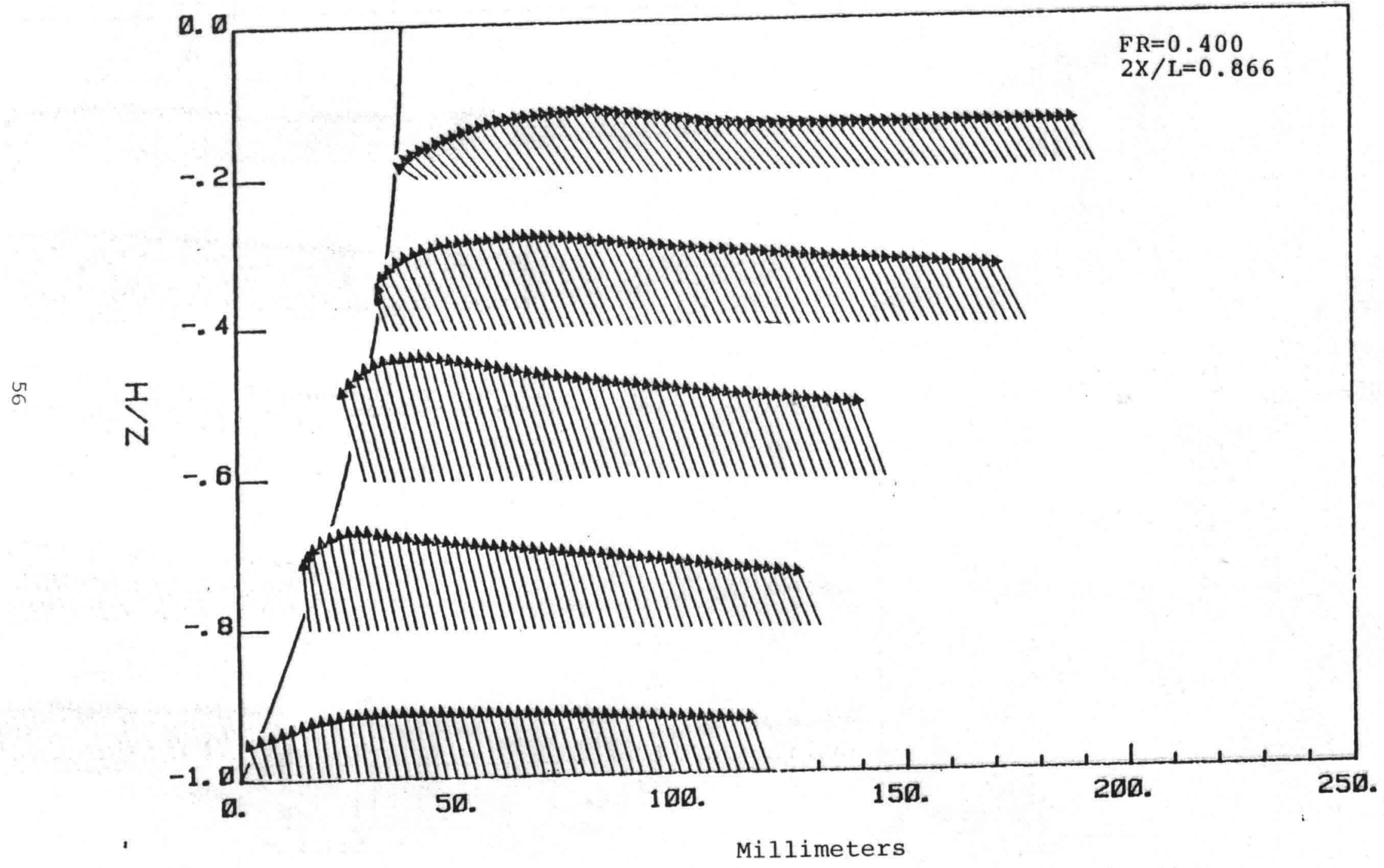

Figure 28. Vector plot of $W-v$ components $(2 x / L=0.86, F r=0.400)$. 


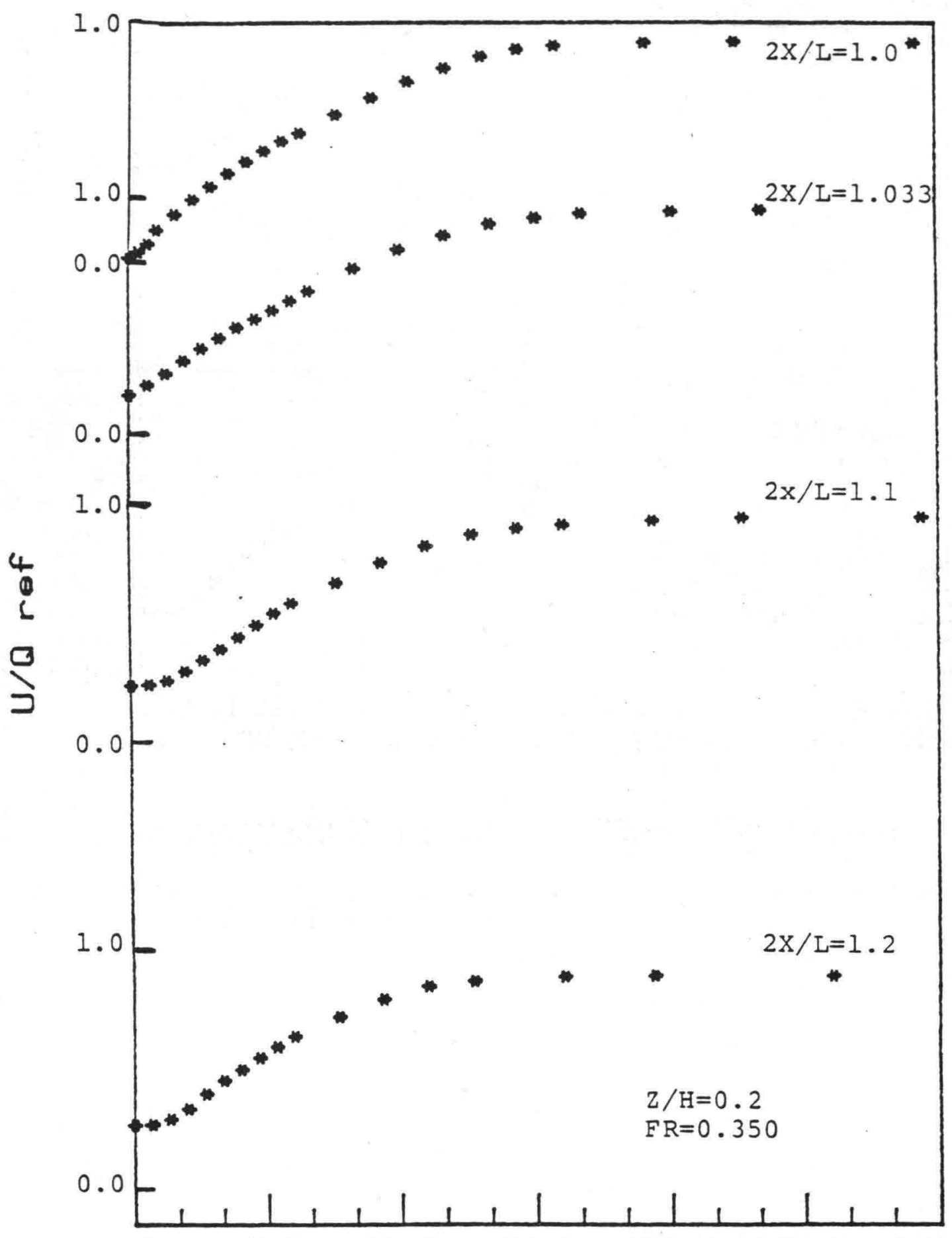

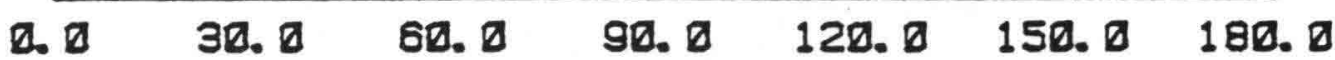

\section{DISTANCE FROM CENTERLINE (mm)}

Figure 29. Measured streamwise velocity component in the wake $(|z / H|=0.2, \mathrm{Fr}=0.350$. 


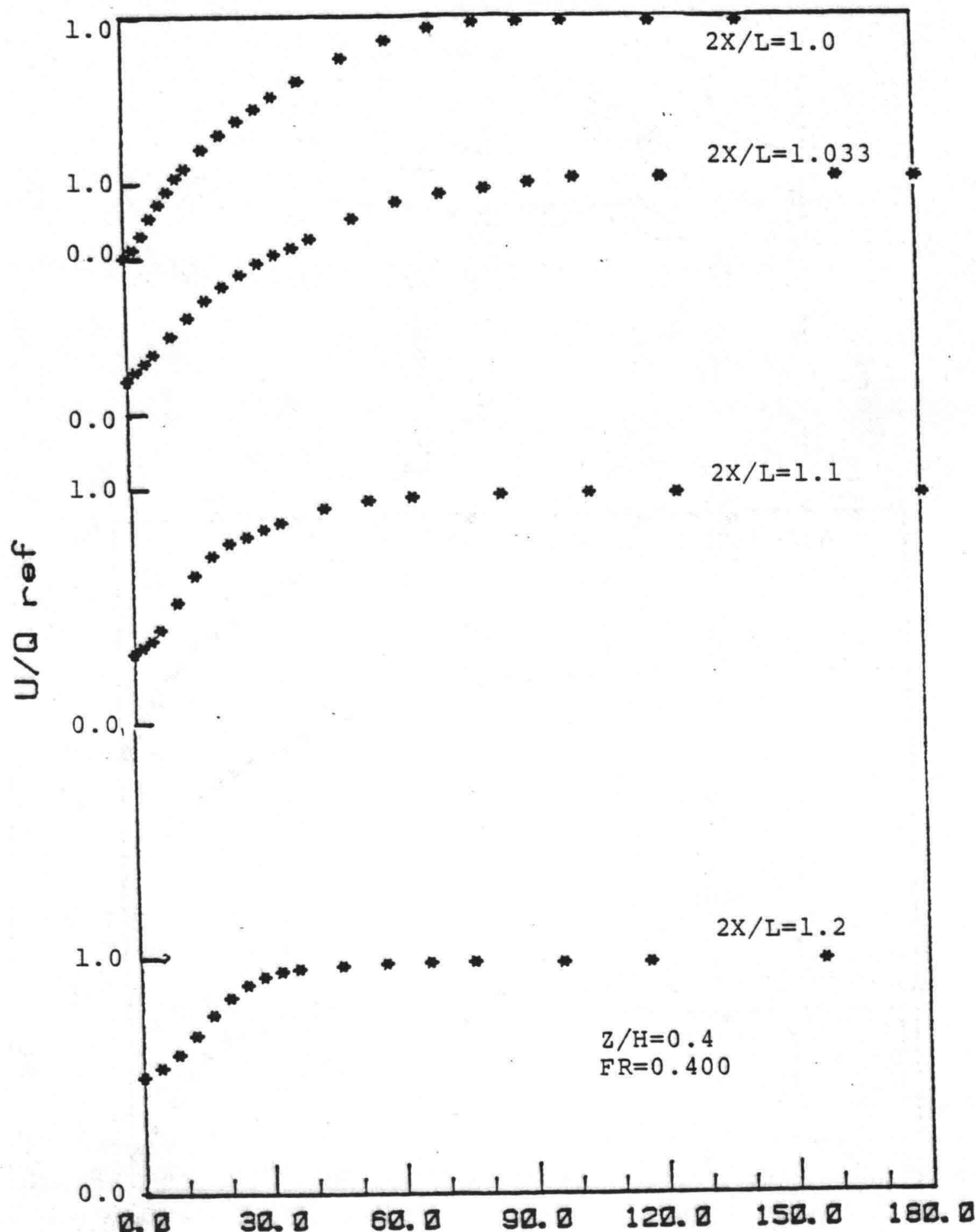

\section{DISTANCE FROM CENTERLINE (mm)}

Figure 30. Measured streamwise velocity component in the wake $(\mid z / H=0.4, F r=0.400)$. 


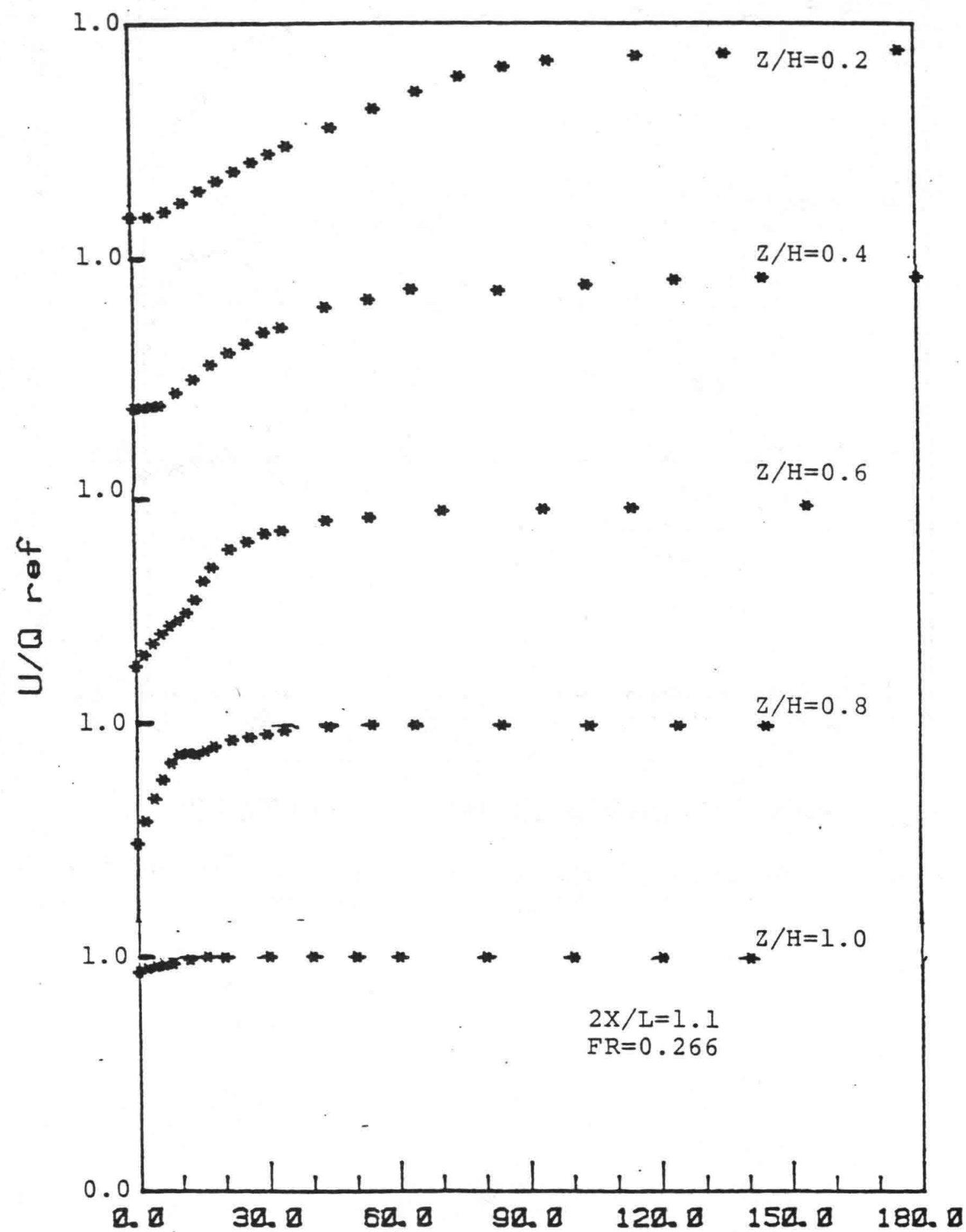

\section{DISTANCE FROM CENTERLINE (mm)}

Figure 31. Measured strearmise velocity component in a cross section $(2 x / L=1.1, \mathrm{Fr}=0.266)$. 


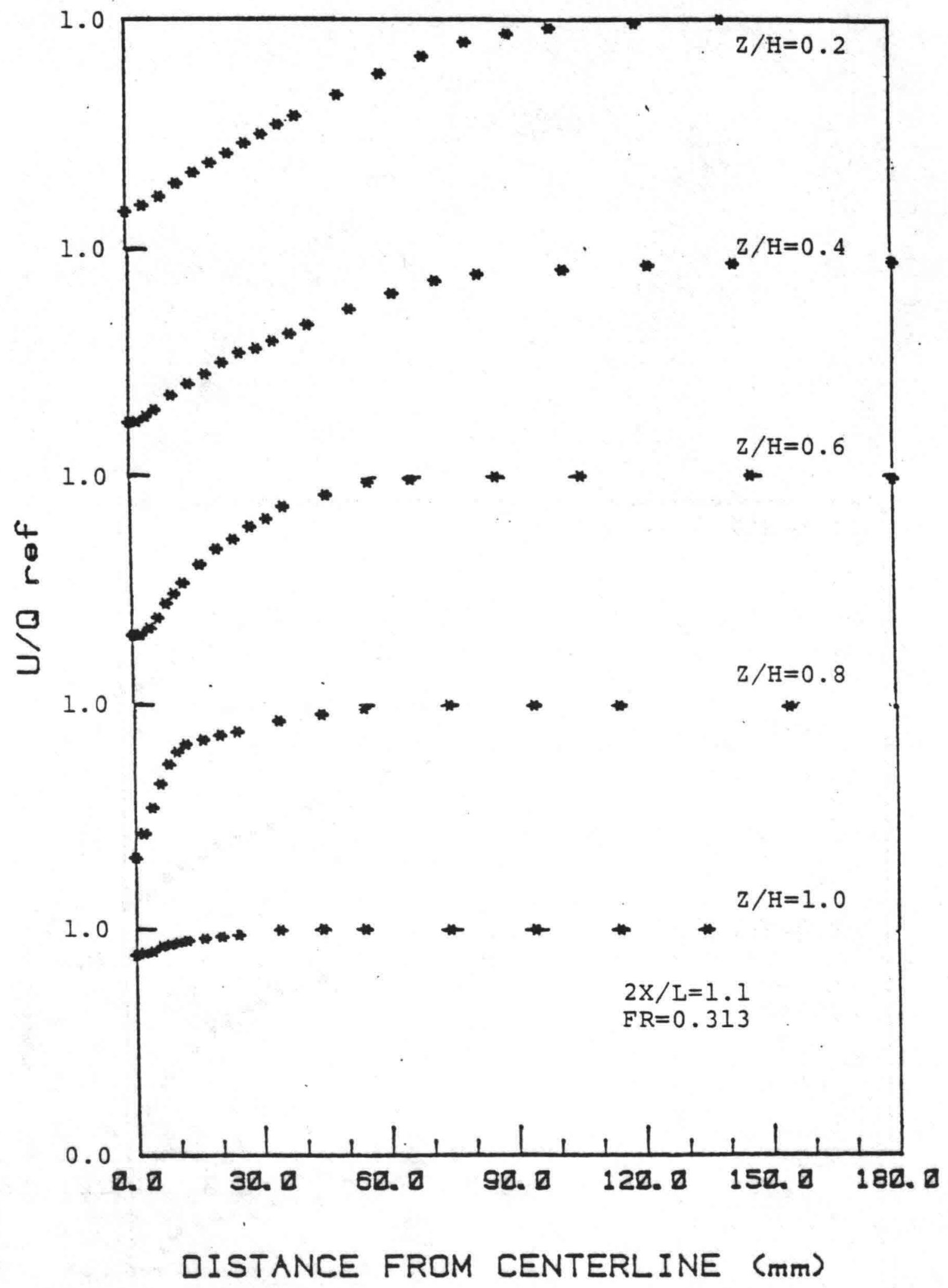

Figure 32. Measured streamwise velocity component in a cross section $(2 x / L=1.1, F r=0.313)$. 


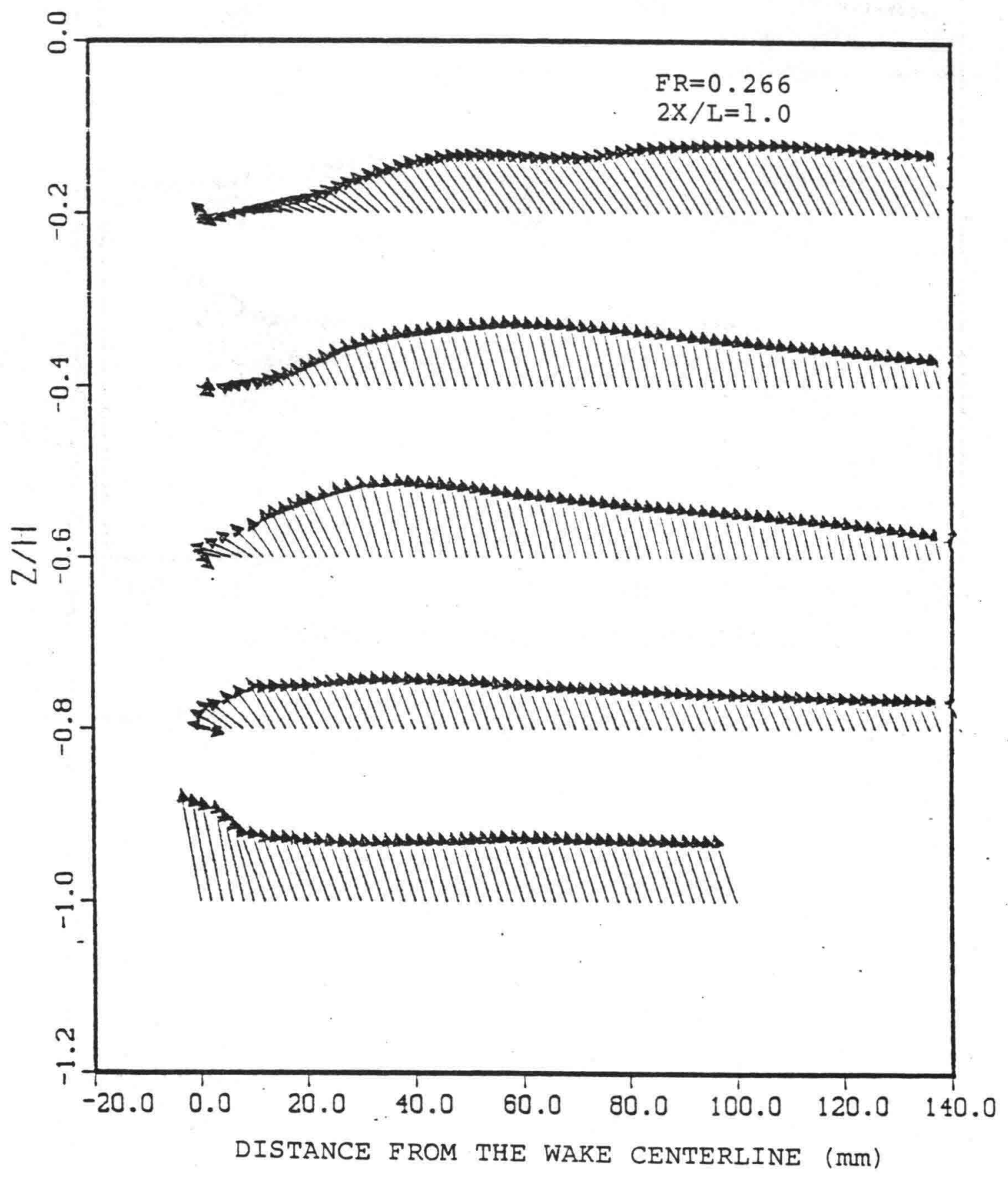

Figure 33. Vector plot of $w-v$ component $(2 x / L=1.0, \mathrm{Fr}=0.266)$. 


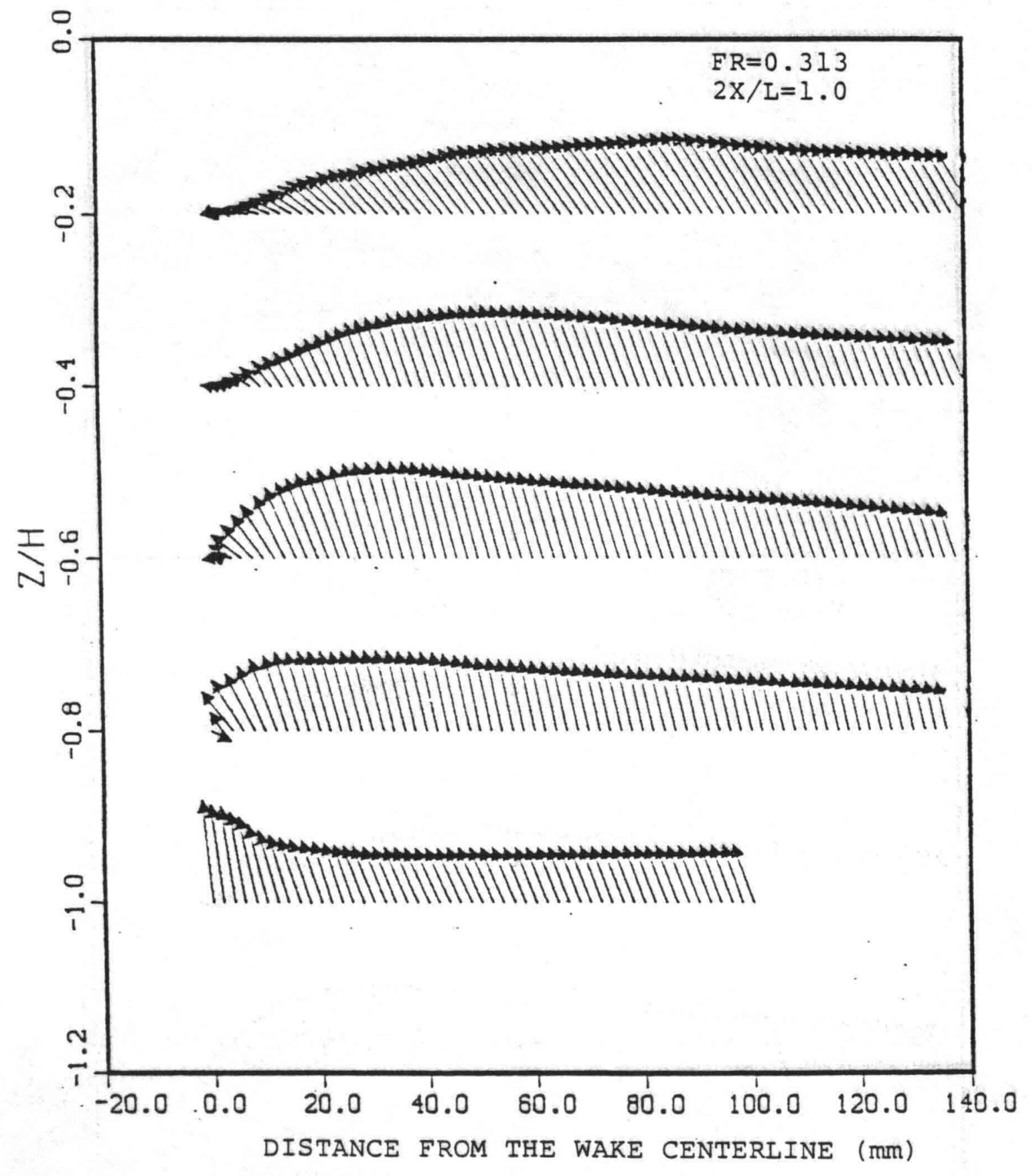

Figure 34. Vector plot of $w-v$ component $(2 x / L=1.0, F r=0.313)$. 


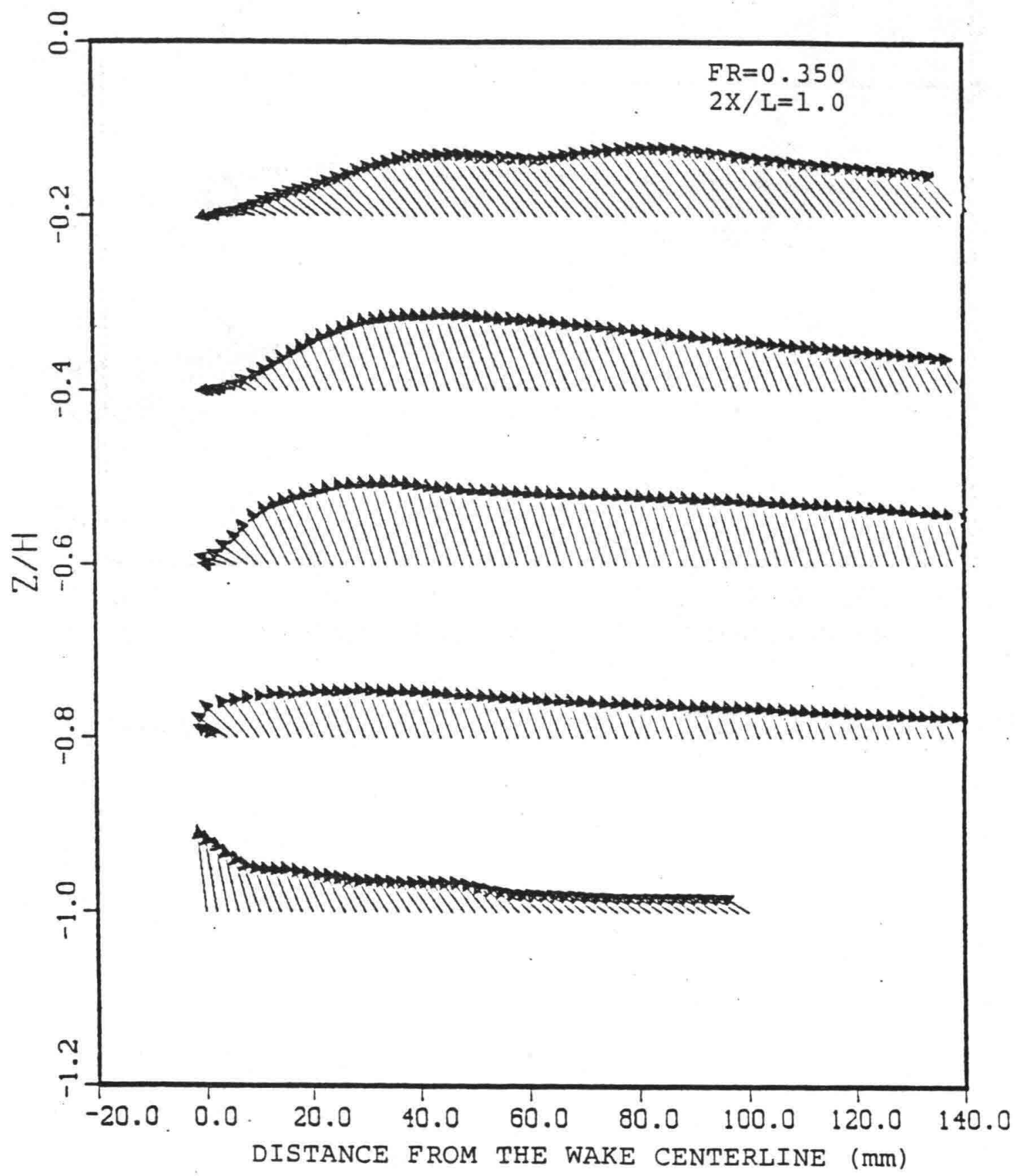

Figure 35. Vector plot of $w-v$ component $(2 x / \mathrm{L}=1.0, \mathrm{Fr}=0.350)$. 


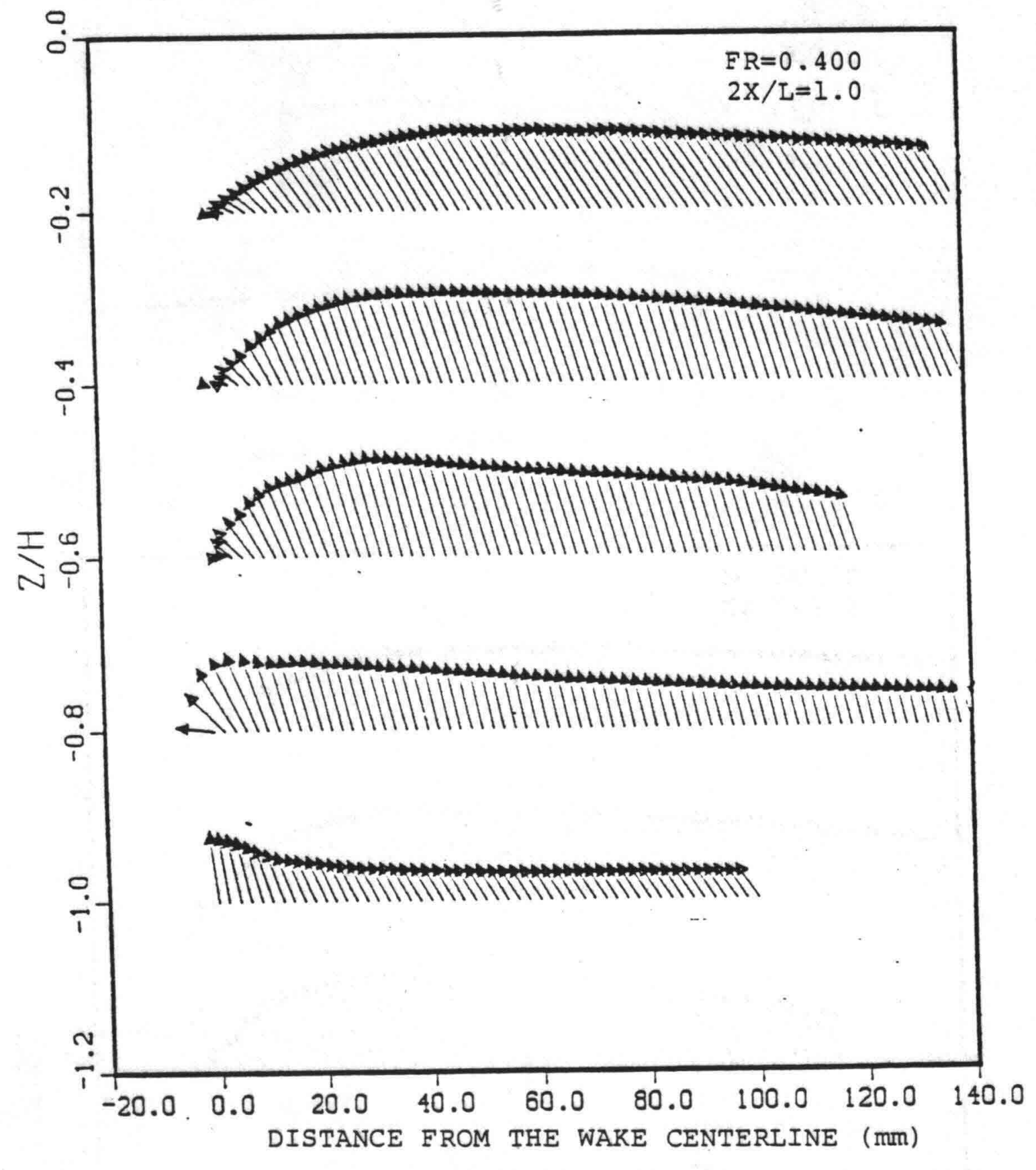

Figure 36. Vector plot of $\mathrm{W}-\mathrm{v}$ component $(2 x / \mathrm{L}=1.0, \mathrm{Fr}=0.400)$. 


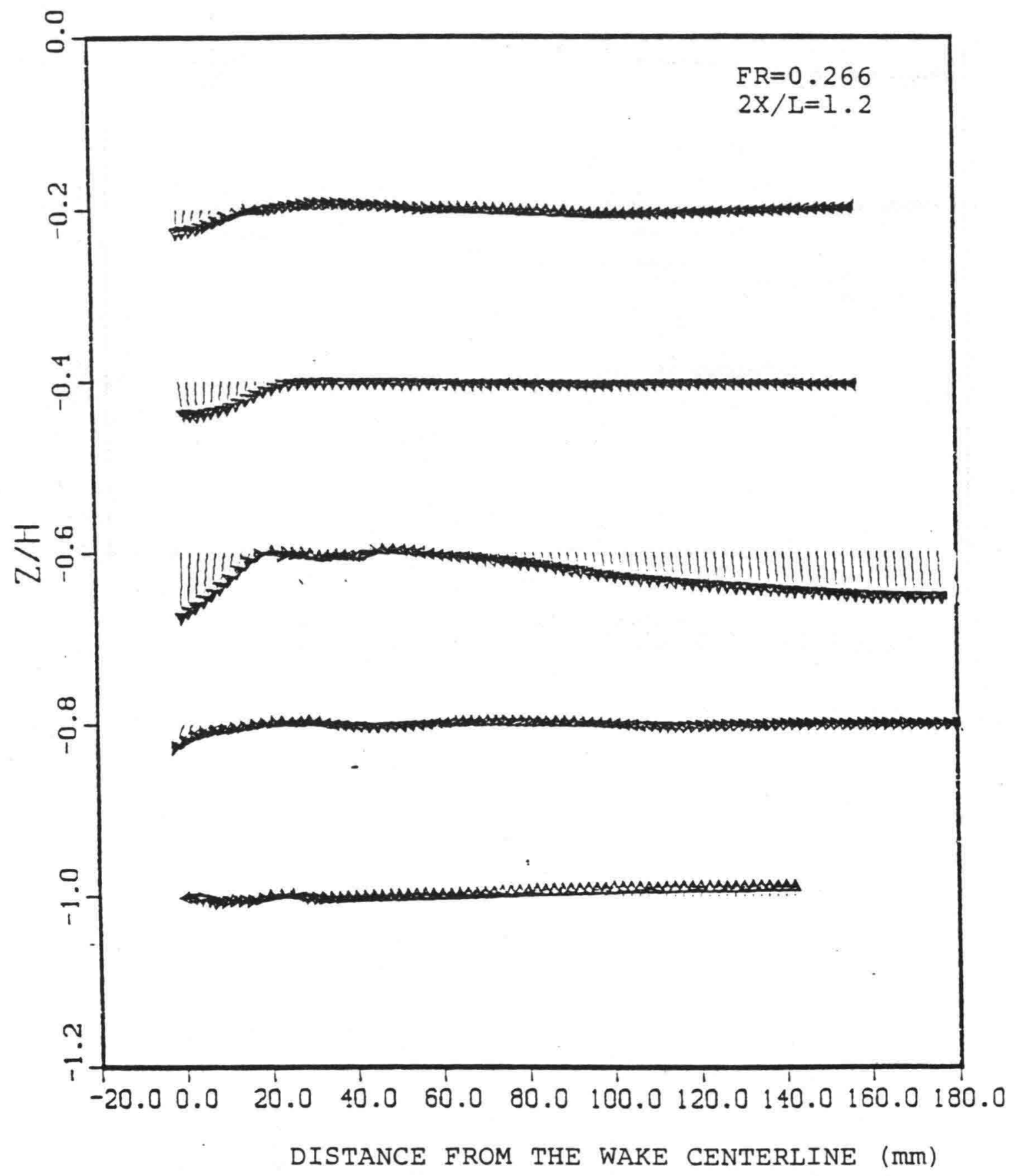

Figure 37. Vector plot of $w-v$ component $(2 x / L=1.2, \mathrm{Fr}=0.266)$. 


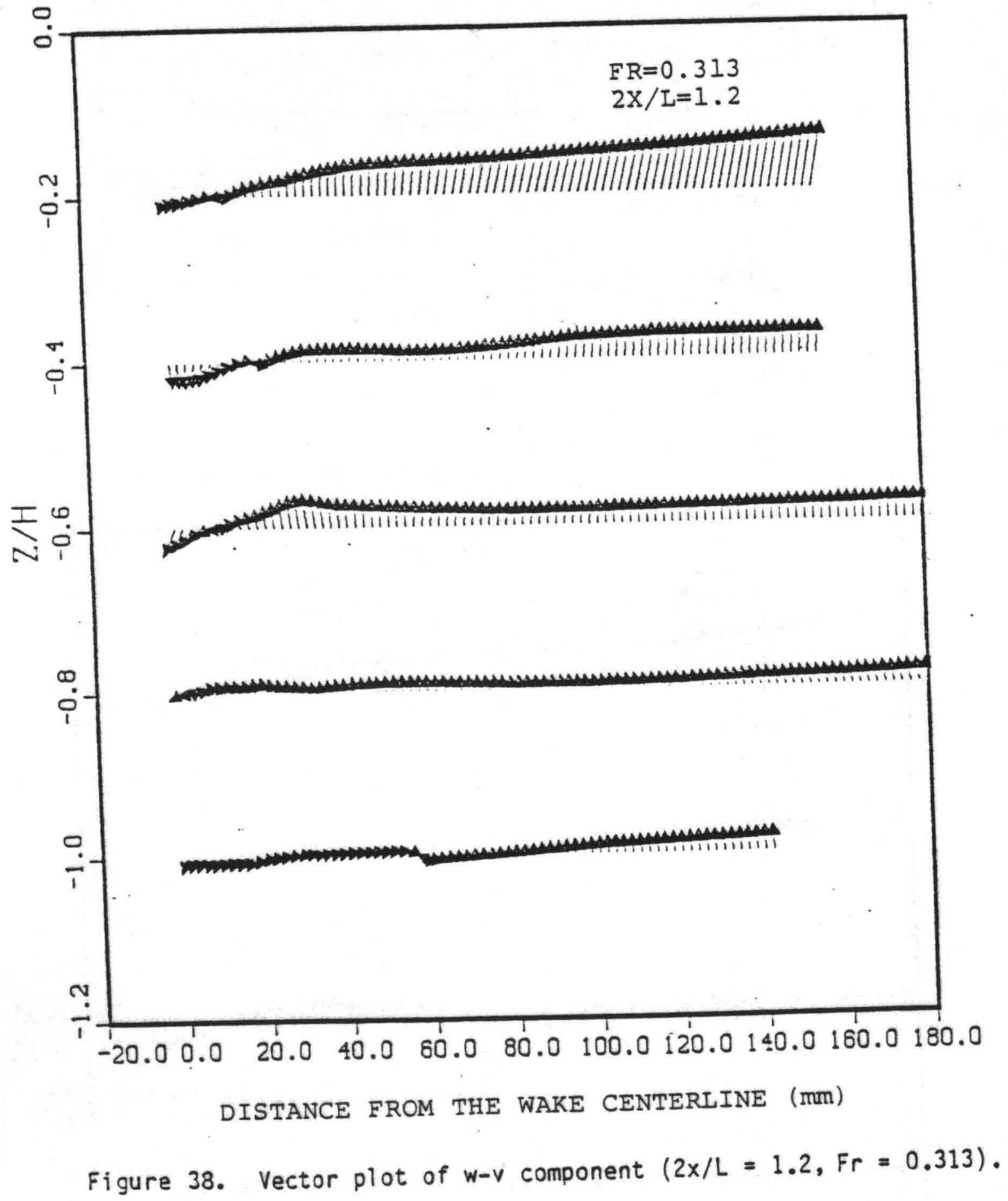




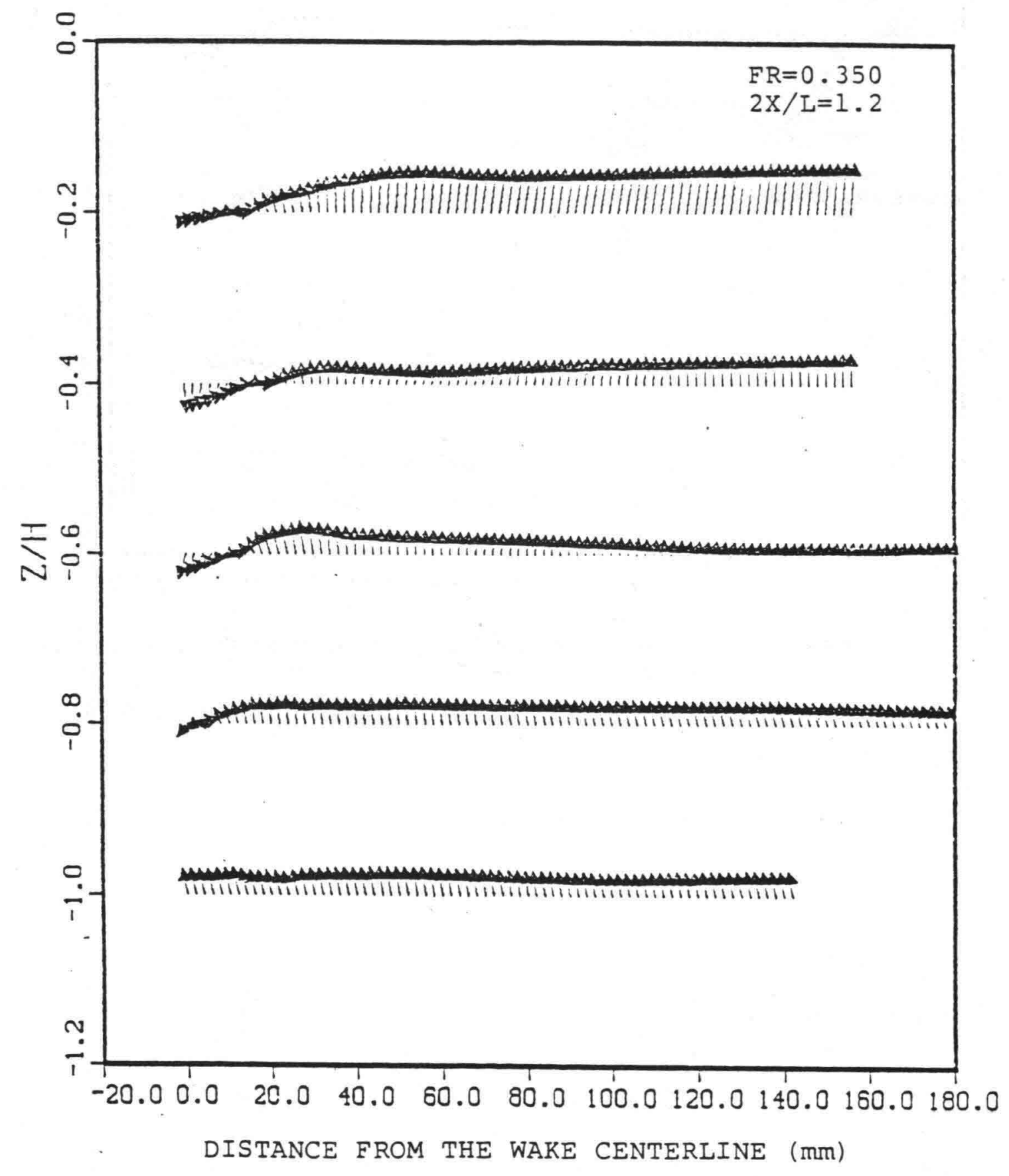

Figure 39. Vector plot of $w-v$ component $(2 x / L=1.2, F r=0.350)$. 


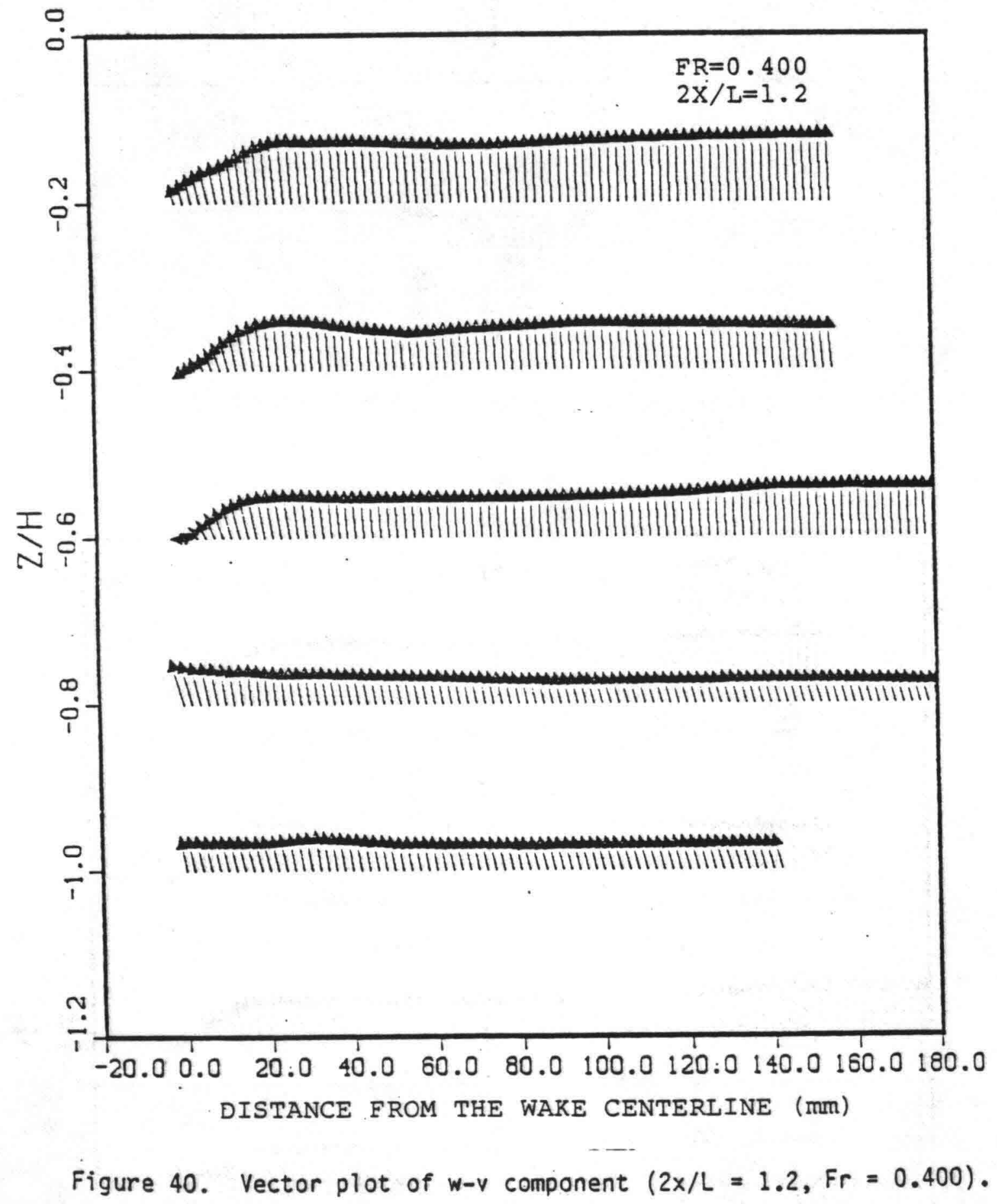




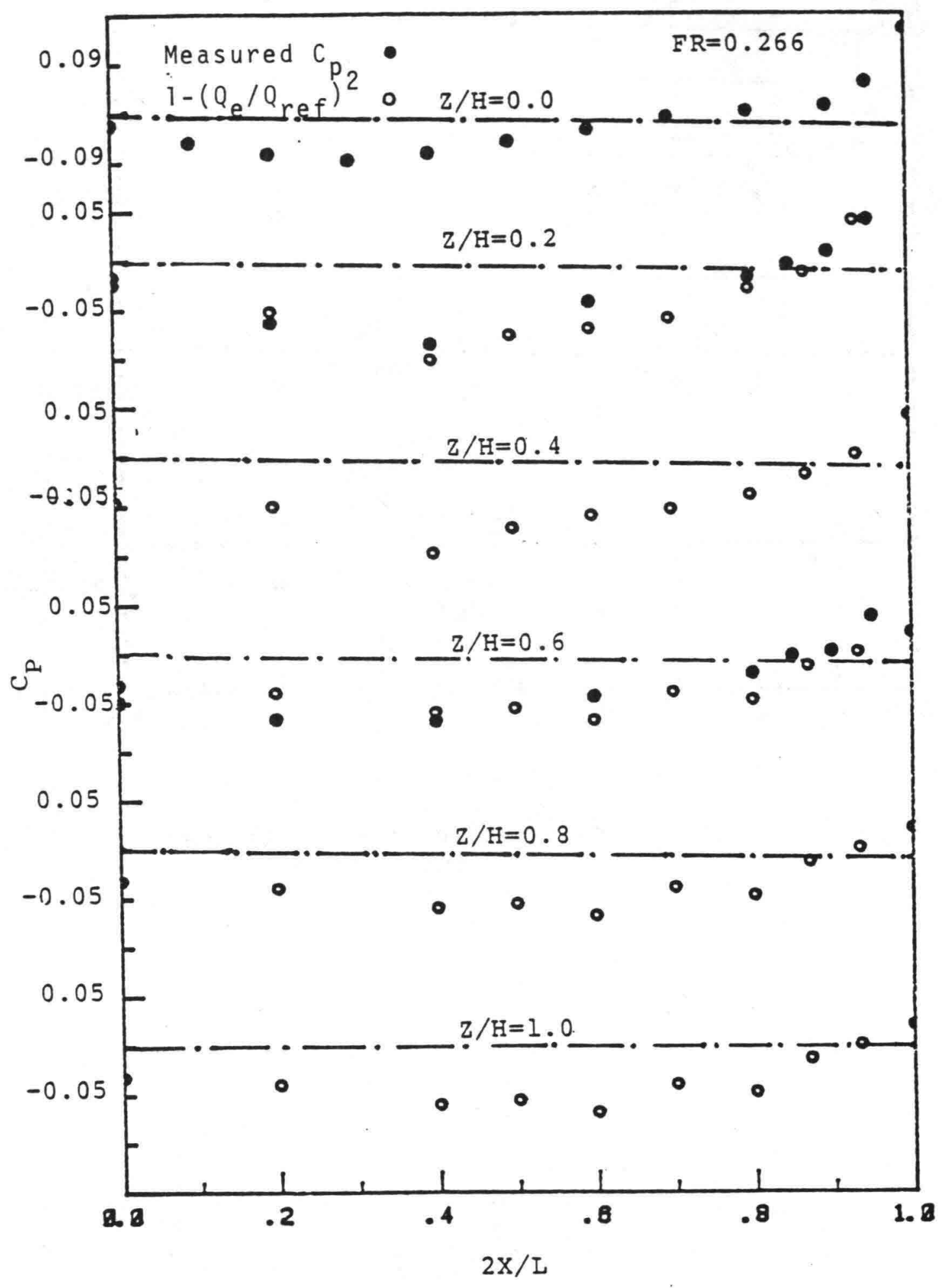

Figure 41. Comparison of measured $C_{p}$ and $1-\left(Q_{e} / Q_{\text {ref }}\right)^{2}$ $(F r=0.266)$. 


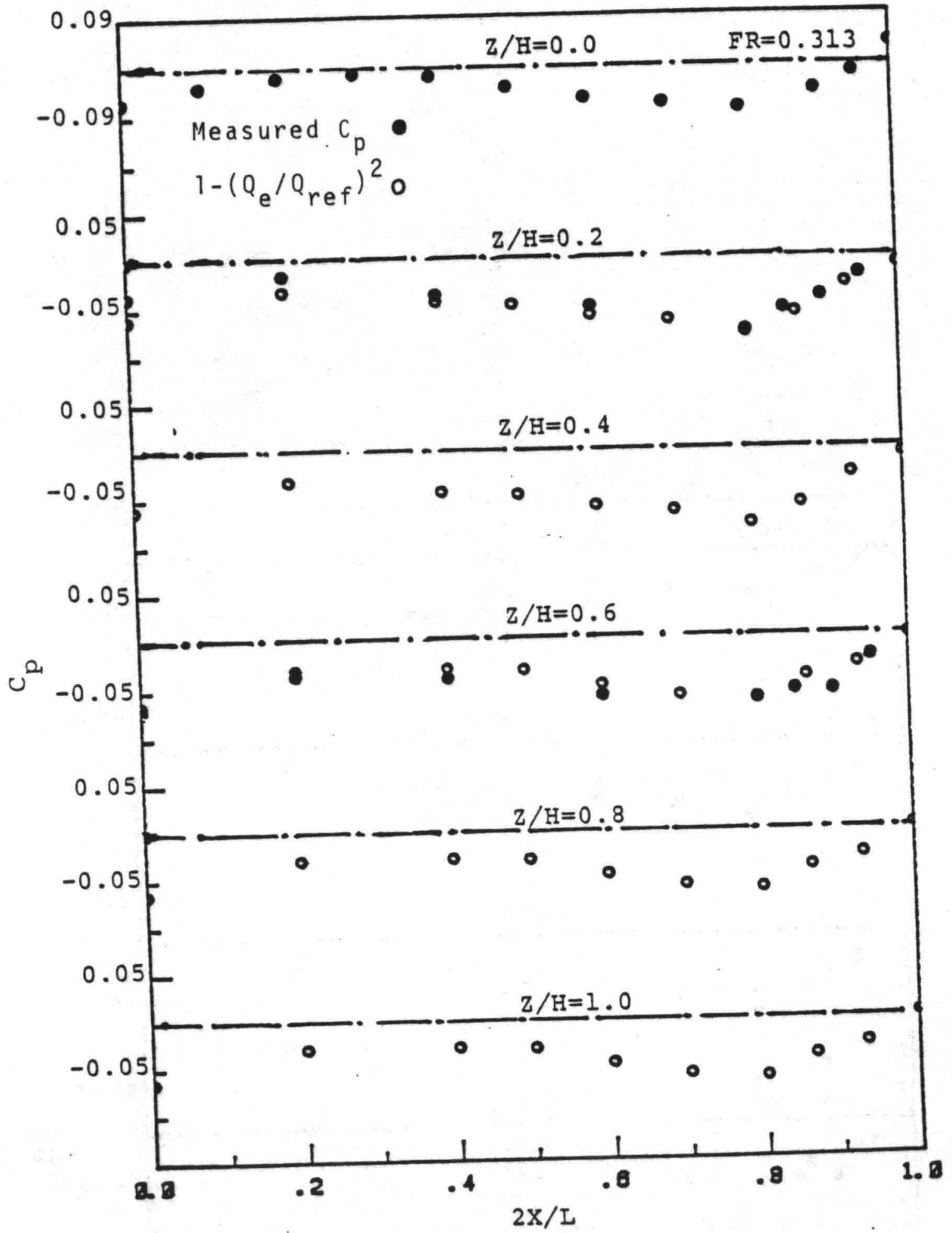

Figure 42. Comparison of measured $C_{p}$ and $1-\left(Q_{e} / Q_{\text {ref }}\right)^{2}$ $\left(F_{r}=0.313\right)$. 


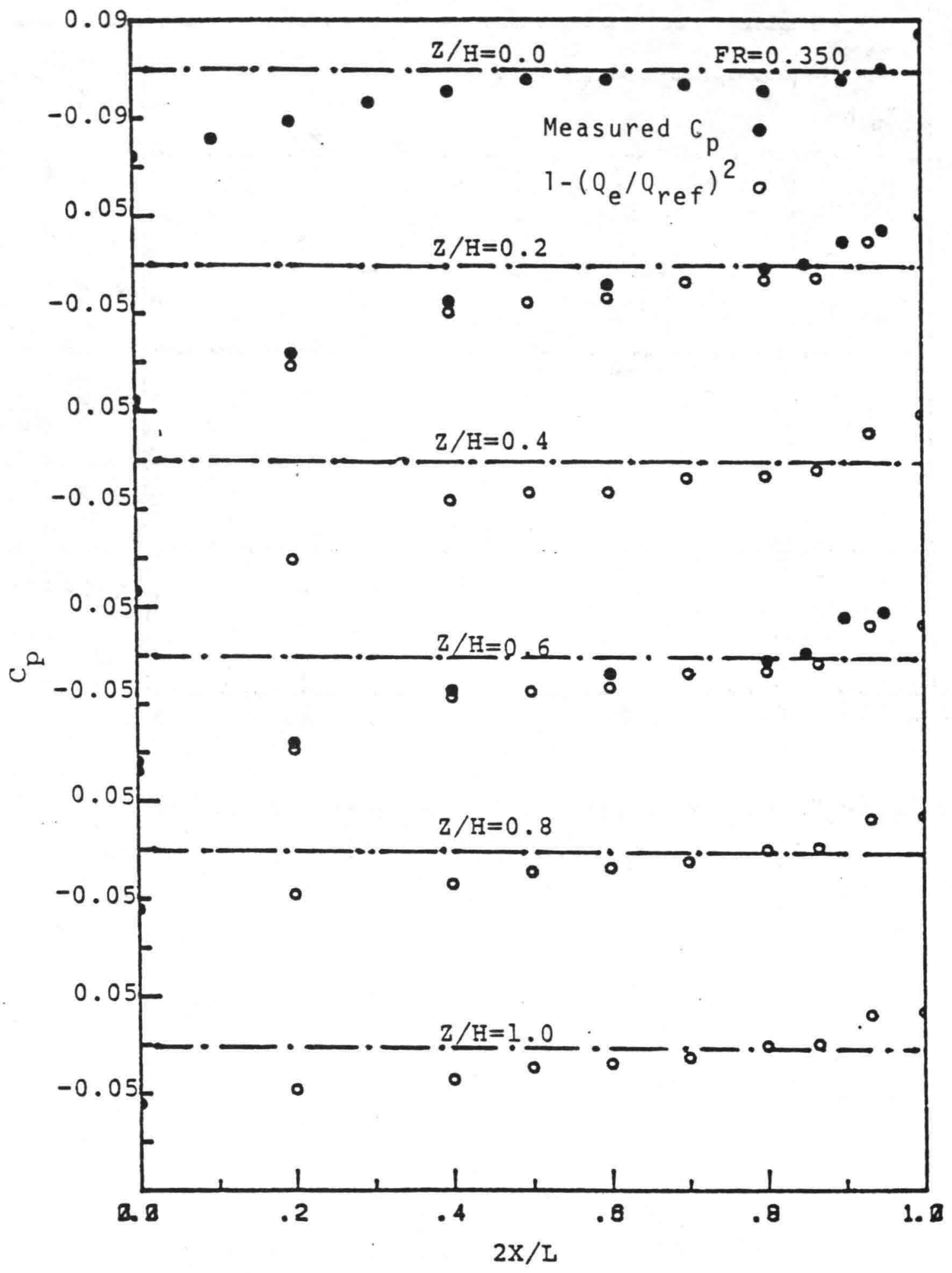

Figure 43. Comparison of measured $C_{p}$ and $1-\left(Q_{e} / Q_{\text {ref }}\right)^{2}$
$(F r=0.350)$. 


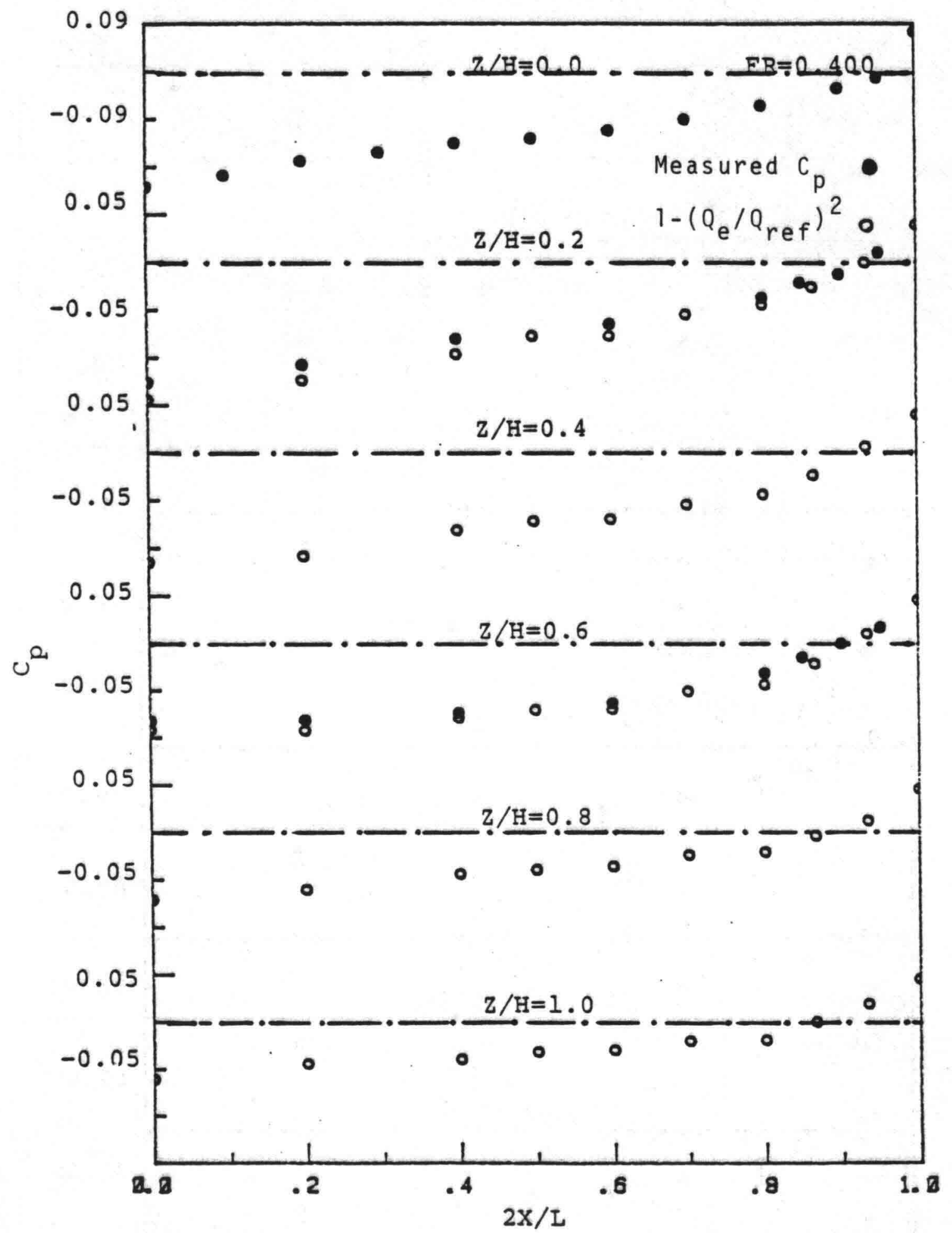

Figure 44. Comparison of measured $C_{p}$ and $1-\left(Q_{e} / Q_{\text {ref }}\right)^{2}$
$(\mathrm{Fr}=0.400)$. 


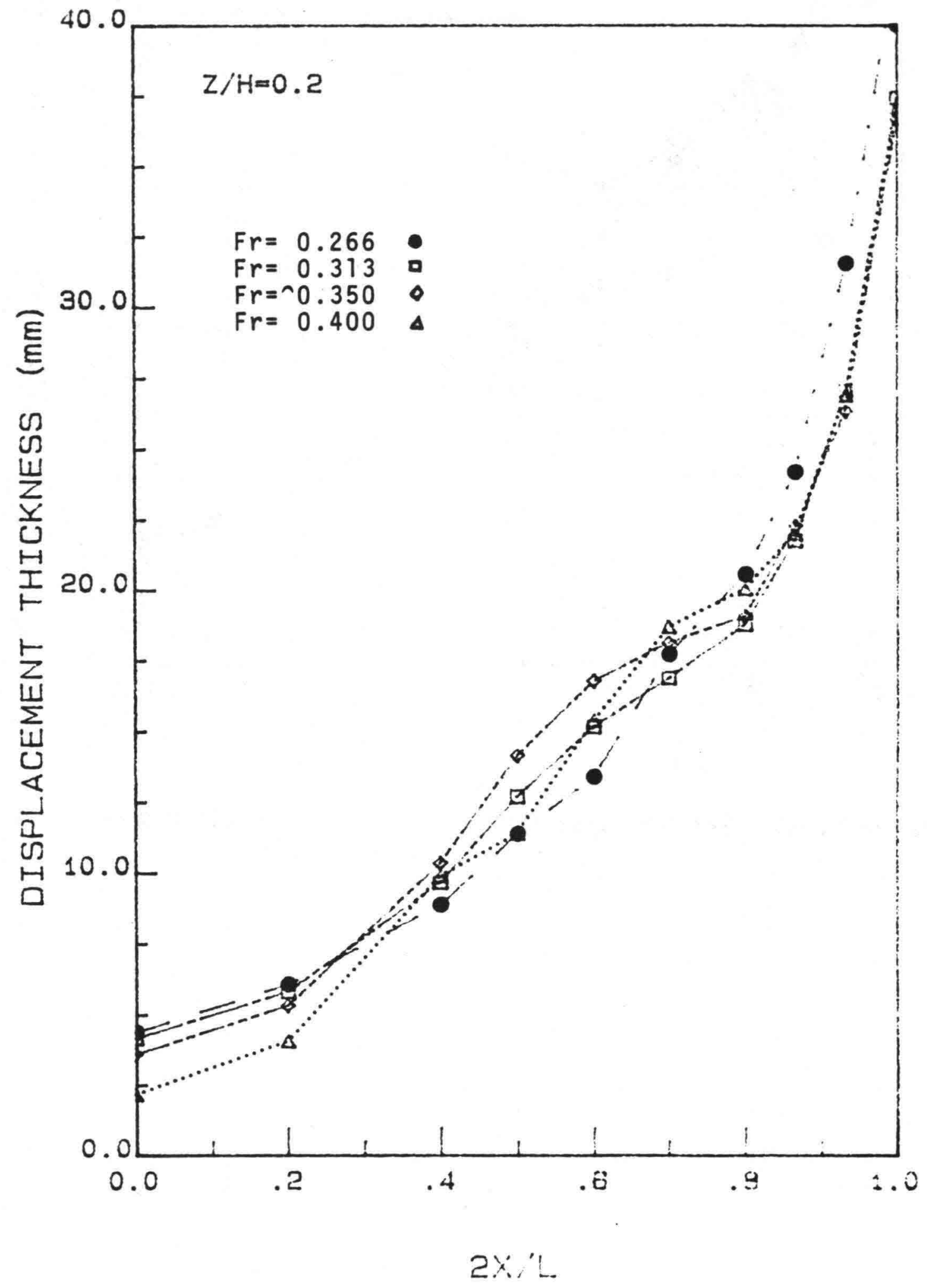

Figure 45. Comparison of displacement thickness along the hull $(|z / H|=0.2)$. 


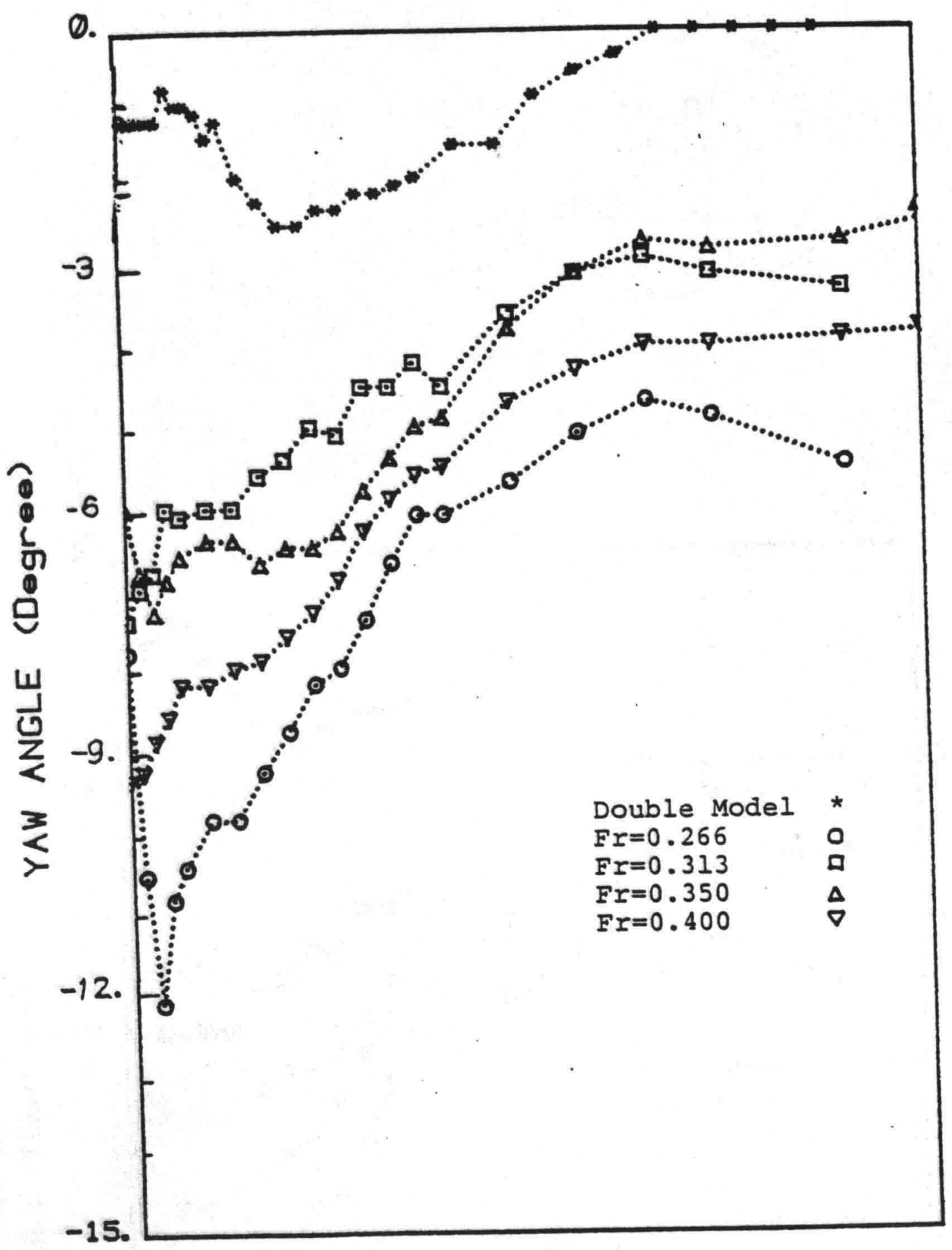

(Y/L)*1日D

Figure 46. Comparison of the yaw angles across the boundary layer $(2 x / L=0.8,|z / H|=0.2)$. 


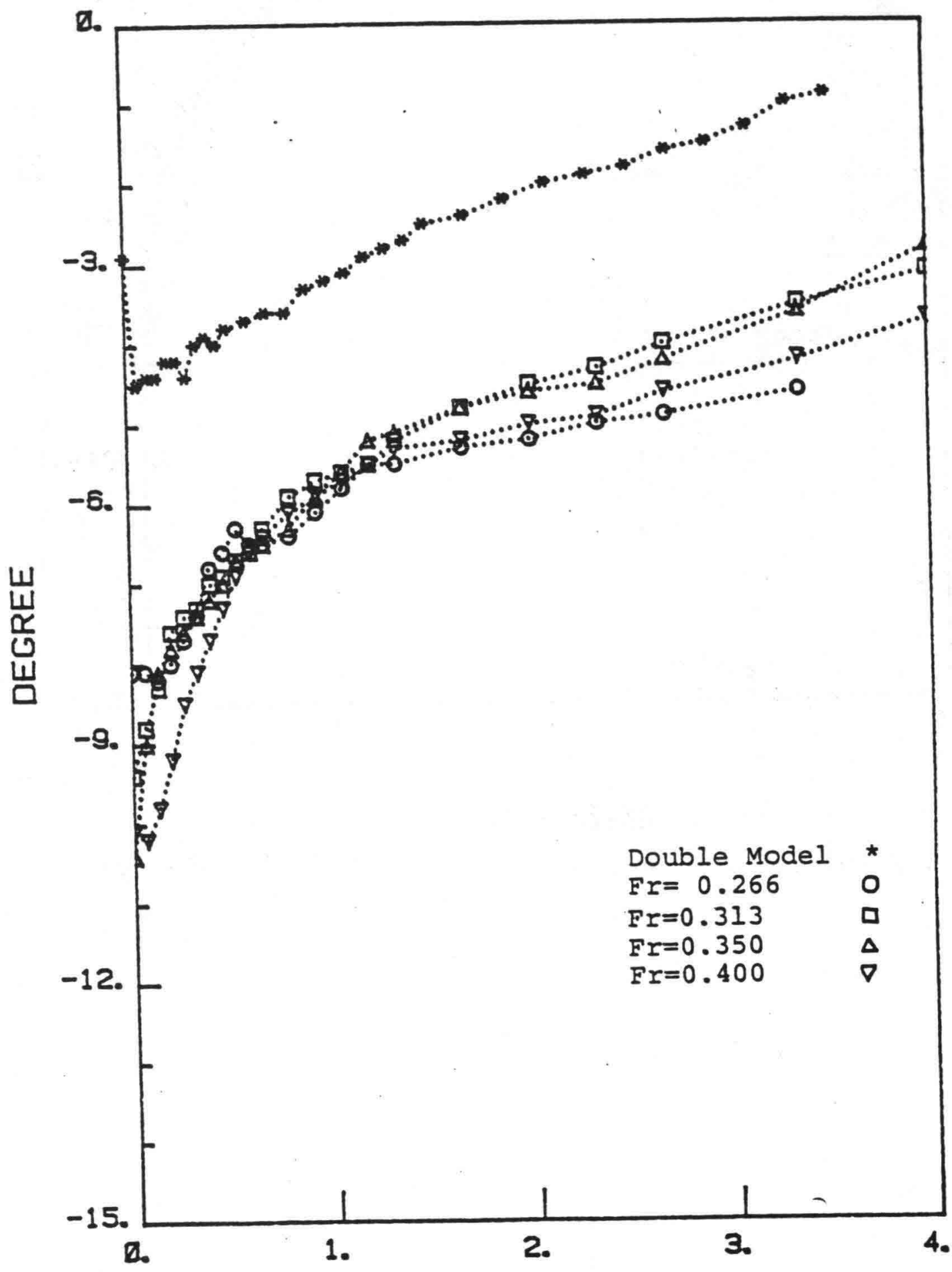

$(Y / L) * 1 \boxminus \square$

Figure 47. Comparison of the yaw angles across the boundary layer $(2 x / L=0.8,|z / H|=0.8)$. 


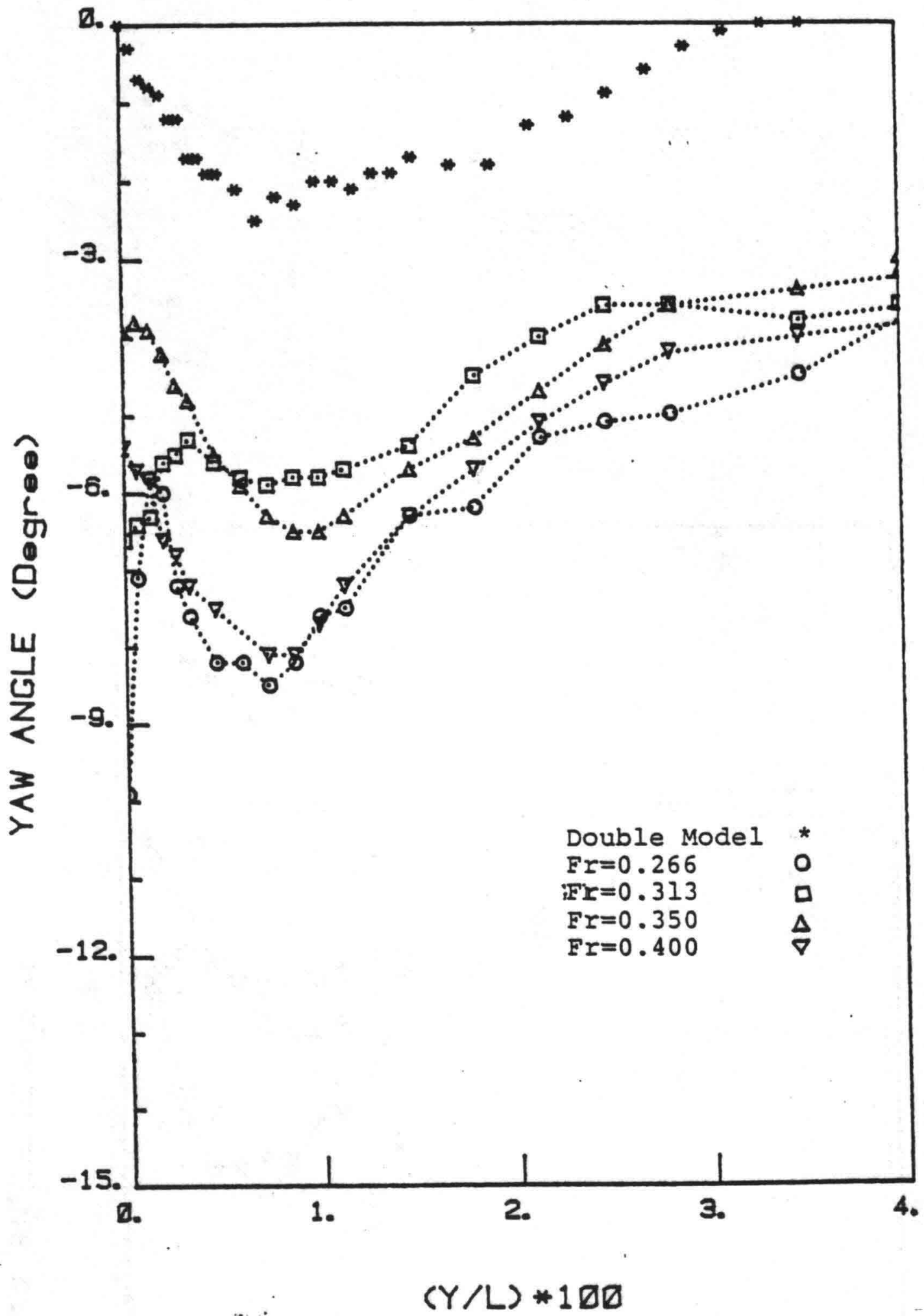

Figure 48. Comparison of the yaw angles across the boundary layer $(2 x / L=0.933,|z / H|=0.2)$. 


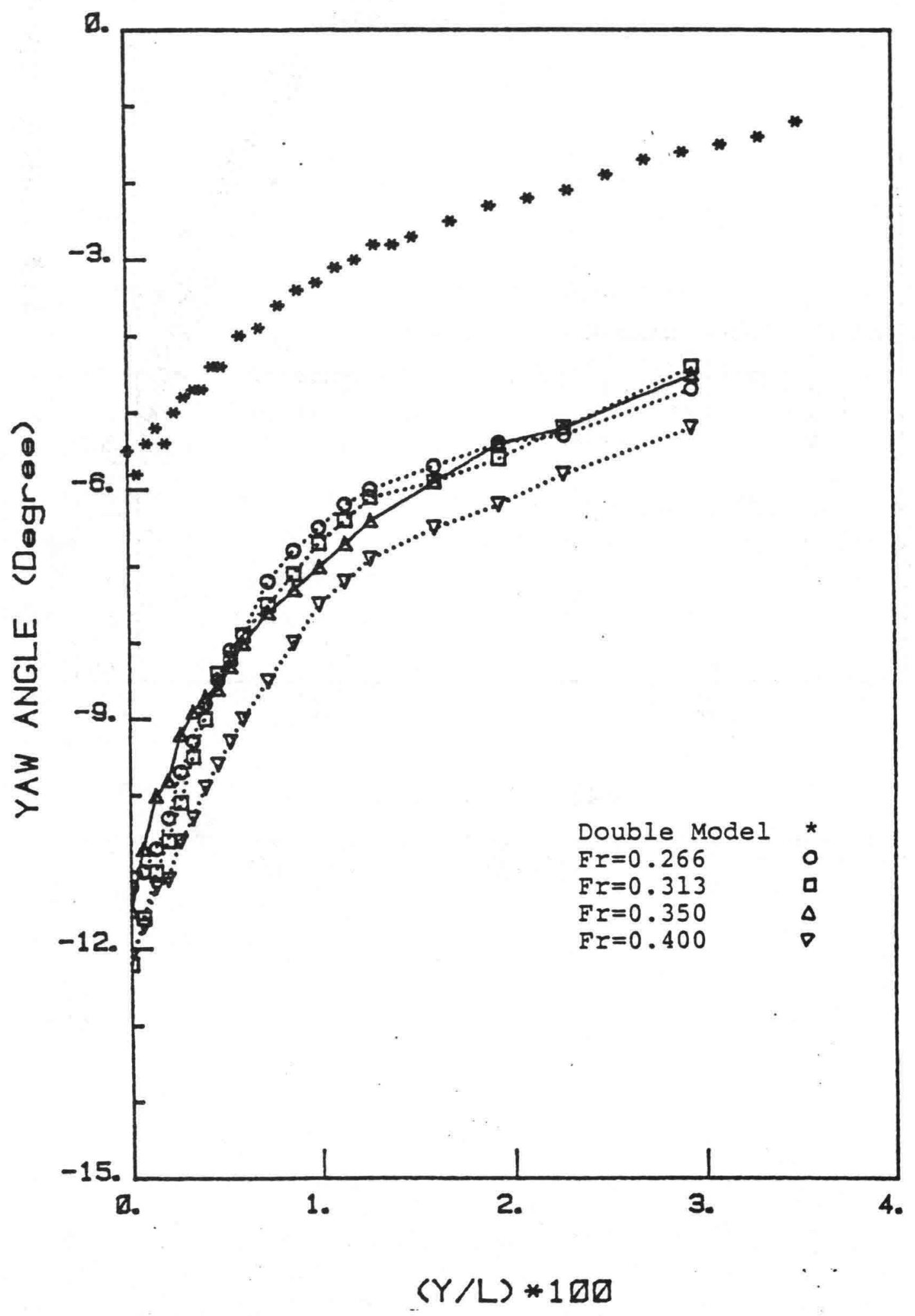

Figure 49. Comparison of the yaw angles across the boundary layer $(2 x / L=0.933,|z / H|=0.8)$. 
CHAPTER IV

BOUNDARY-LAYER CALCULATIONS FOR THE WIGLEY HULL

\section{General}

The problem of predicting ship viscous resistance has intrigued physicists, mathematicians, and engineers for many years. The inadequacy of the so-called Froude method, in which the viscous resistance is taken as the frictional resistance of an equivalent flat plate with an empirical-shape factor correction, has been demonstrated experimentally (Landweber and Wu, 1963). Reliable and accurate methods for determining the viscous resistance should necessarily involve the solution of the three-dimensional boundary-layer equations. In view of the difficulties of calculating the pressure distribution on a ship form at a nonzero Froude number, however, the computation of the boundary-layer development on ship hulls seems a formidable task. Due to this complexity, three dimensional boundary layers on ship hulls are often calculated by considering the double model, i.e., the zero-Froude-number case. The influence of the free surface on the boundary layer is therefore neglected. A review of these methods is given by Landweber (1971), and Landweber and Patel (1979). The aforementioned simplification suffers from several drawbacks. First, the pressure distribution on the double body is independent of Froude number. In the nonzero case, however, the pressure distribution is a function of the Froude number. Consequently, the boundary layer over the hull is not modeled correctly by double-body calculations. Secondly, the changes in the wetted area of the hull, associated with different wave patterns, are ignored and therefore the influence of Froude number on viscous resistance cannot be calculated. Finally, any local separation due to wave steepness along the hull (Chow, 1967), cannot be predicted.

As a first step toward taking a wavy free surface into account, Shahshahan and Patel (1983) considered the boundary layer along the intersection between the hull and the free surface and demonstrated the phenomenon of local wave-induced separation at the free surface. In this chapter, their method has been employed to calculate the development of the boundary layer on 
the wigley hull. The numerical results have then been compared with the experimental results obtained in the towing tank.

\section{Coordinate System and Governing Equations}

\subsection{Coordinate System}

Consider the ship hull with its surface is given by $y= \pm f(x, z)$ in rectangular Cartesian coordinates $(x, y, z)$, with increasing values of $x$ in the direction opposite to the ship's forward motion, z vertically upward, and the origin at midship at the undisturbed free surface. Denote any streamline on the hull by $z=g(x)$. This streamline is assumed to be known either from experiment or inviscid-flow theory. For the hull boundary layer along this streamline an orthogonal coordinate system $(\xi, \eta, \zeta)$ has been chosen, in which $\xi$ is measured along the streamline, $n$ is normal to the hull ( $n=0$ at the hull surface), and $\zeta$ is the third member of this right-handed, orthogonal coordinate system. See Figure 50. It should be noted that this coordinate system is triply orthogonal only on the hull surface, and it is not exactly orthogonal off the hull since the $\xi$ and $\zeta$ lines do not coincide with the lines of principal curvature of the surface. The departures from orthogonality of the coordinate system, however, will be small since the boundary layer is assumed to be thin.

In the present calculation, the measured wave profile at the hull was used as the first streamline. Other streamlines along the hull below the free were calculated by using Guilloton's (1948, 1951) method. This method has been used extensively by many researchers, e.g., Emerson (1967), Webster and Huang (1970), Gadd (1973), and Adachi and Hinatsu (1981), and indications are that the resulting streamlines are in good agreement with measurements. The single drawback is that only a few streamlines can be obtained by this procedure. Figures 51 through 54 show the measured wave profile and four streamlines which were obtained for the Wigley hull at Froude numbers 0.266, 0.313, 0.350 , and 0.400 , respectively. 


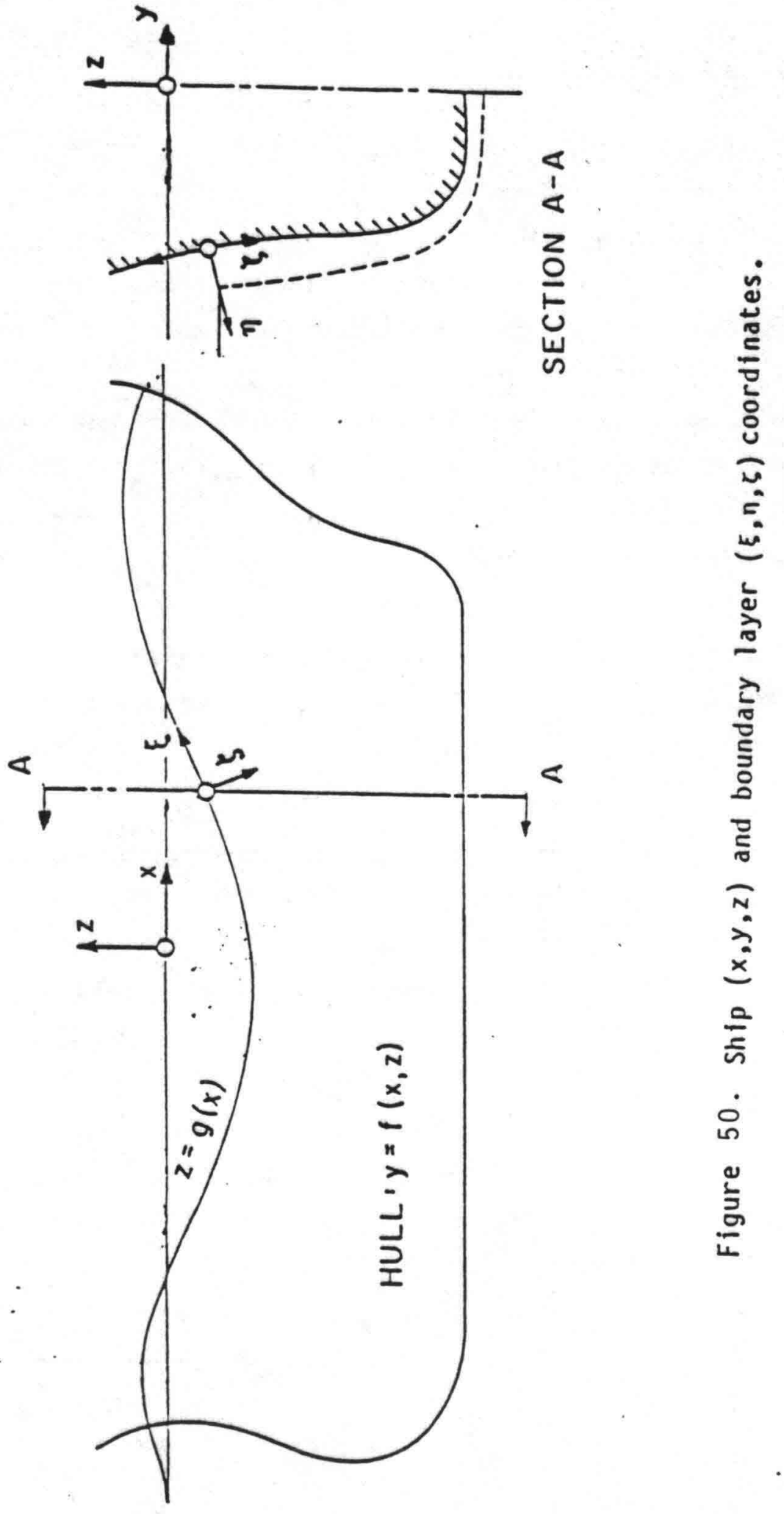




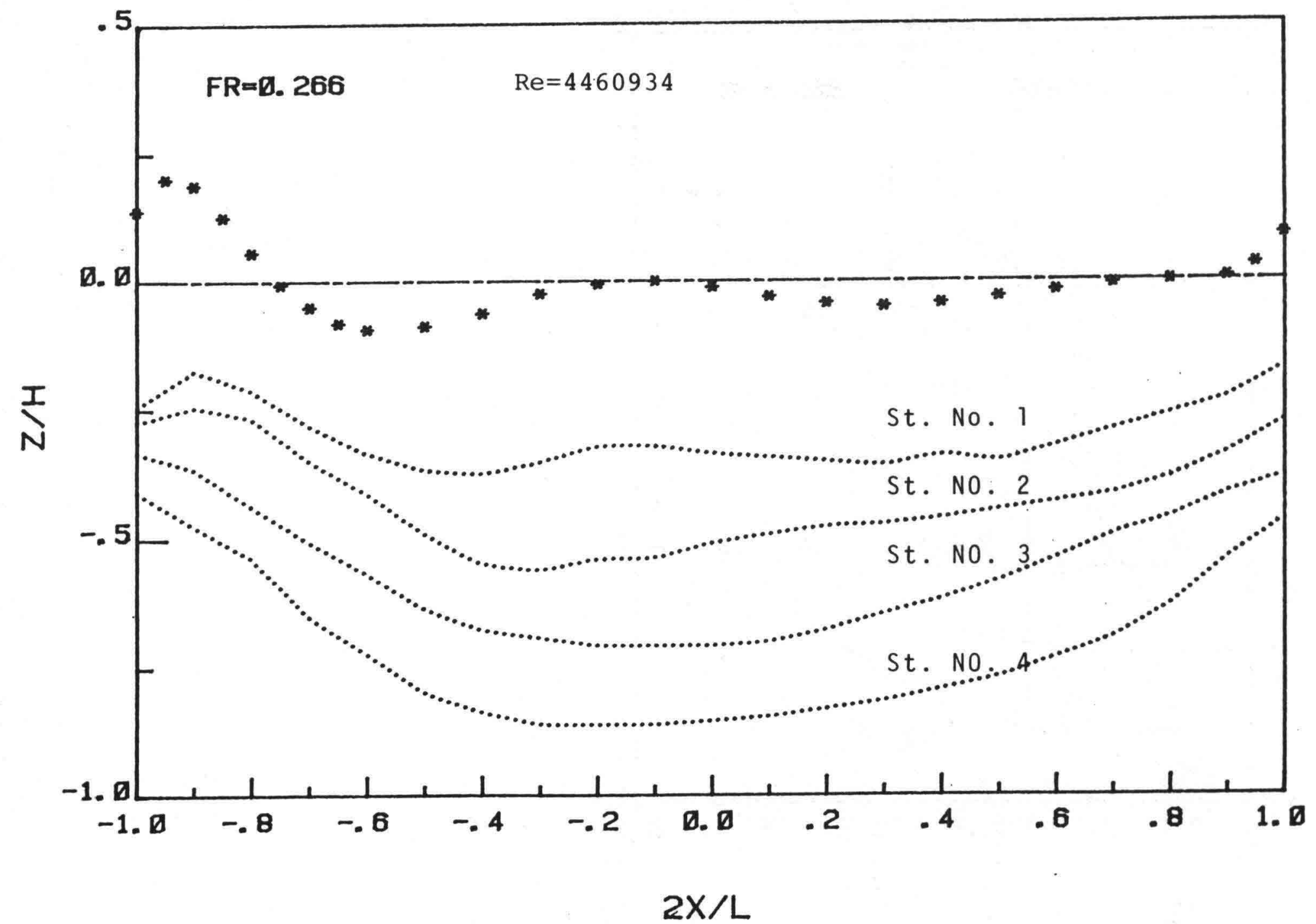

Figure 51. Measured wave profile and Guilloton's streamline $(\mathrm{Fr}=0.266)$. 


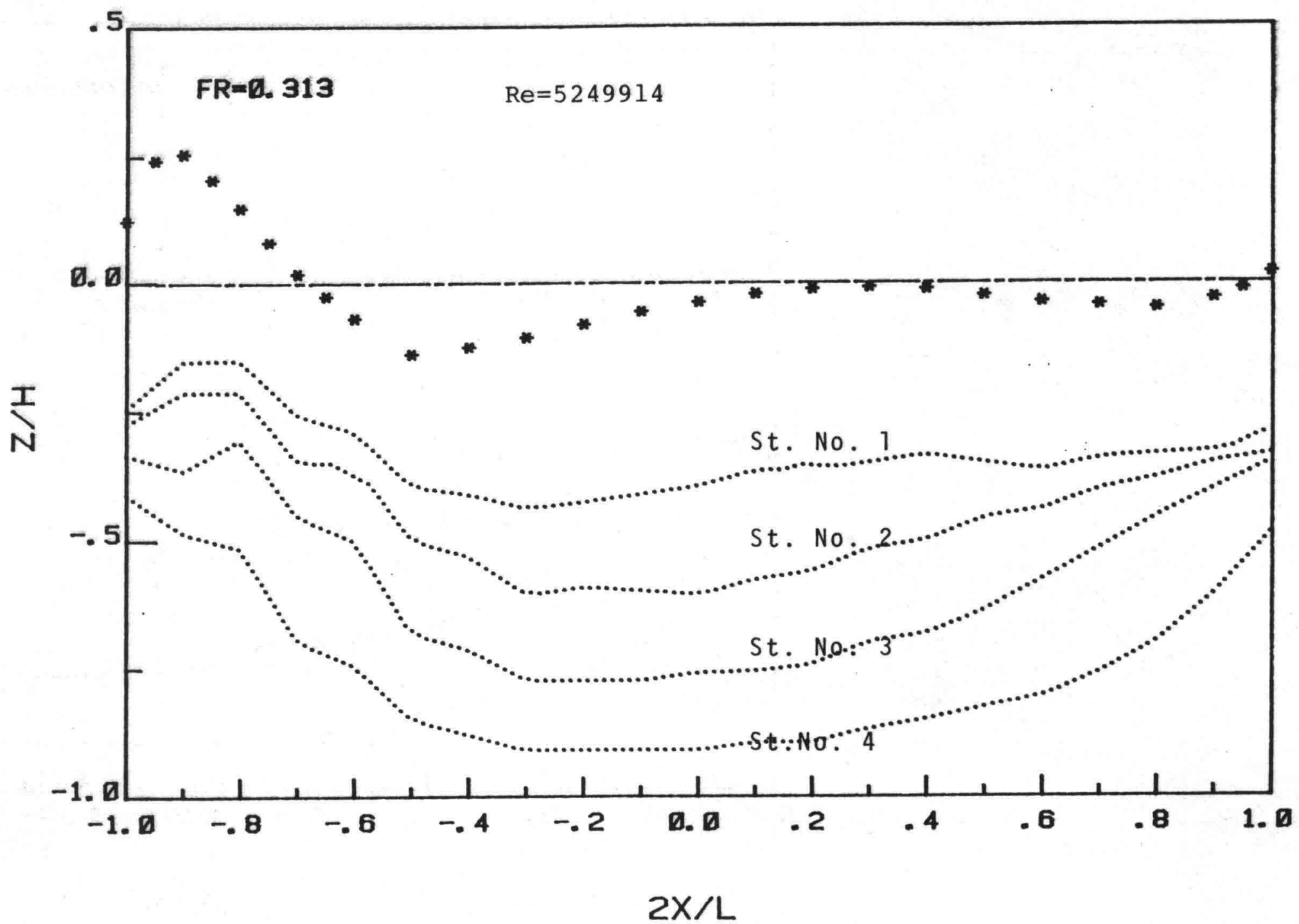

Figure 52. Measured wave profile and Guilloton's streamline $(\mathrm{Fr}=0.313)$. 


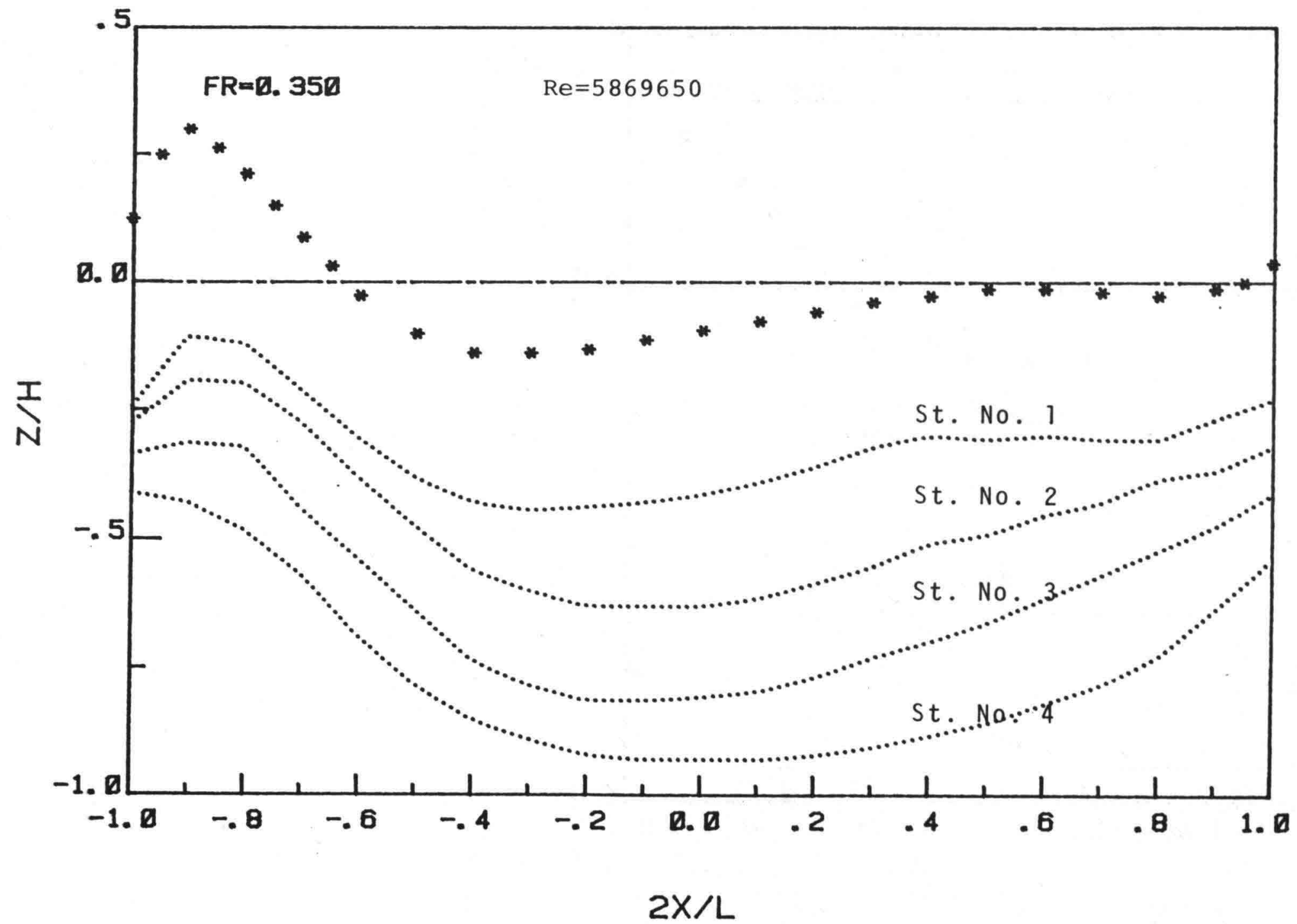

Figure 53 . Measured wave profile and Guilloton's streamline $(\mathrm{Fr}=0.350)$. 


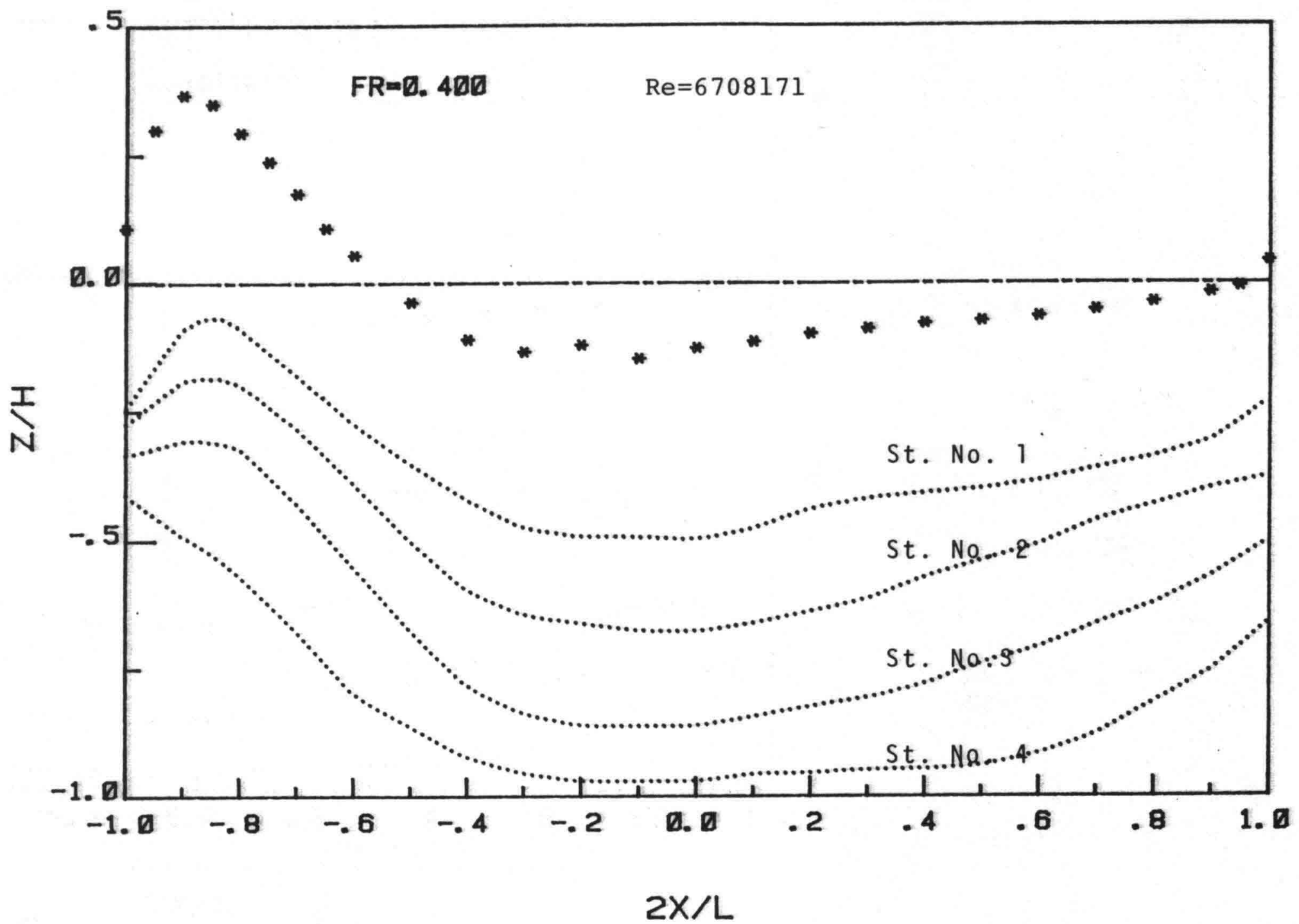

Figure 54. Measured wave profile and Guilloton's streamline $(\mathrm{Fr}=0.400)$. 


\subsection{Governing Equations}

One of the most common assumptions, which has been used extensively in boundary-layer calculations applicable to ship forms, is the assumption of sinall crossflow, i.e., the direction of flow within the boundary layer deviates by only a small angle from the direction of the streamline at the outer edge of the boundary layer. The validity of this assumption, however, has been questioned; Landweber (1971). The combination of the hull geometry and the secondary flow generated by the wave profile along the hull, particularly around the bow and stem where the wave profile is the steepest, results in a large crossflow velocity component for most hulls. Therefore the smallcrossflow assumption seems unsuitable. For the Wigley hull, however, this is not the case. The boundary-layer measurements obtained for this model show that the crossflow angle $\left(\beta=\tan ^{-1} \frac{\mathrm{w}}{\mathrm{u}}\right)$ reaches a maximum value of about eight degrees for the steepest wave profile. Thus one may assume that the boundary layer over the Wigley hull can be calculated by using the small-crossflow equations (see Nash and Patel (1972))

$\frac{U}{h_{\xi}} \frac{\partial U}{\partial \xi}+v \frac{\partial U}{\partial \eta}-\frac{U_{e}}{h_{\xi}} \frac{\partial U_{e}}{\partial \xi}+\frac{\partial}{\partial \eta}(\overline{u v})-\nu \frac{\partial^{2} U}{\partial \eta^{2}}=0$

$\frac{1}{h_{\xi}} \frac{\partial U}{\partial \xi}+\frac{\partial V}{\partial \eta}+K_{31} U=0$

$\frac{U}{h_{\xi}} \frac{\partial W}{\partial \xi}+v \frac{\partial W}{\partial \eta}+K_{31} W U+K_{13}\left(U_{e}^{2}-U^{2}\right)+\frac{\partial}{\partial \eta}(v w)-v \frac{\partial^{2} W}{\partial \eta^{2}}=0$

where $(U, V, W)$ are components of mean velocity in the $(\xi, n, \zeta)$ directions, $\rho \overline{u v}$ and $\rho \overline{v w}$ are Reynolds stresses, $\nu$ is the kinematic viscosity, and $U_{e}$ is the velocity in the $\xi$ direction outside the boundary layer.

The metric coefficients $h_{\xi}, h_{\zeta}$ and the coordinate curvatures

$$
K_{13}=\frac{1}{h_{\xi} h_{\zeta}} \frac{\partial h_{\xi}}{\partial \zeta}, K_{31}=\frac{1}{h_{\zeta} h_{\xi}} \frac{\partial h_{\zeta}}{\partial \xi}
$$

can be related to the hull geometry $f(x, z)$, and the streamline equation $g(x)$ using the method of Miloh and Patel (1973) as follows:

$$
h_{\xi}^{2}=\left(1+f_{x}^{2}\right)+2 f_{x} f_{z} g^{\prime}+\left(1+f_{z}^{2}\right) g^{\prime^{2}}
$$




$$
\begin{aligned}
& h_{\zeta}^{2}=\left(1+f_{x}^{2}\right) h^{\prime 2}+2 f_{x^{\prime}} f^{\prime \prime}+\left(1+f_{z}^{2}\right) \\
& K_{13}=-\frac{g^{\prime \prime}+D^{2}\left(f_{z}-g^{\prime} f_{x}\right)\left(f_{x x}+2 g^{\prime} f_{x z}+g^{\prime 2} f_{z z}\right)}{D h_{\xi_{n}}^{3}{ }^{2}} \\
& K_{31}=-\frac{\left.h^{\prime \prime}+D^{2}\left(h^{\prime} f_{z}-f_{x}\right) f^{\prime} f_{x x}+2 h^{\prime} f_{x z}+f_{z z}\right)}{D h_{\zeta}^{2}}
\end{aligned}
$$

where

and

$$
D=\left(1+f_{x}^{2}+f_{z}^{2}\right)^{-1 / 2}
$$

$$
h^{\prime}=\frac{g^{\prime}+\left(f_{x}+g^{\prime} f_{z}\right) f_{z}}{1+\left(f_{x}+g^{\prime} f_{z}\right) f_{x}}
$$

Equation (17) expresses the orthogonality of the $\zeta$ and $\xi$ directions.

The assumptions of small crossflow enable the boundary layer along each streamline to be calculated independently. This argument is also valid at the free surface. The assumption that the deviations of the $\zeta$ coordinate line from the normal to the free surface across the boundary layer are small, i.e., the deflections of the free surface across the boundary layer are small, then leads to the usual small-crossflow approximations and enables the boundary layer along the wave profile to be calculated independently of the flow below the free surface.

\section{Solution Procedure and the Results}

\subsection{Solution Procedure}

The small-crossflow assumption uncouples the continuity equation (10) and the $\xi$-momentum equation (9) from the $\zeta$-momentum equation (11). Hence equations (9) and (10) can be solved for $U$ and V, and then the crossflow W can be determined from equation (11). These equations have been solved by the CrankNicolson method of Chang and Patel (1975). The Reynolds stresses are obtained from the two-layer eddy-viscosity model, and transition, wherever applicable, is simulated by an intermittency function. See Cebeci and Smith (1976).

Boundary-layer calculations along the wave profiles on the hull were started with the Blasius solution a short distance downstream of the bow and transition was imposed at five percent of the length, i.e., $15.24 \mathrm{~cm}$ from the bow, where the model was fitted with plastic studs. The streamwise velocity 
at the edge of the boundary layer, $U_{e}$, was determined from the wave elevation via the Bernoulli equation

$$
u_{e}^{2}=u_{\infty}^{2}-2 g_{o} g
$$

where $g_{0}$ is the gravity acceleration, $g=g(x)$ is the wave elevation along the hull, and $U_{\infty}$ is the forward speed of the ship.

Calculations along other streamlines were started at midship with the measured velocity, profiles and the flow was considered fully turbulent. The velocity at the edge of the boundary layer along these streamlines, $U_{e}$, was determined from $U_{e}^{2}=u_{e}^{2}+w_{e}^{2}$, where $u_{e}$ and $w_{e}$ are the edge values for velocity components obtained from the measurements.

\subsection{Calculation Results}

Figure 55 shows the measured wave profiles on the hull for four Froude numbers of $0.266,0.313,0.350$, and 0.400 . The distribution of the wall shear stress presented in Figure 56 clearly indicates the influence of waves. The wave system at Froude numbers of 0.266 and 0.313 are strong and produce a marked oscillation in the stress distributions. Note that the usual procedure of double-model calculations would result in a single unrealistic prediction. These calculations also indicate that there is no separation at the free surface in the range of Froude numbers considered. Thus, the influence of Froude number on the viscous resistance is felt primarily through the changes in the friction distribution and the wetted-surface area. Figures 57 through 59 show the variation of displacement thickness, momentum thickness and shape parameter along the wave profiles on the hull. Here again, the effect of Froude number on boundary-layer development is clearly shown by stronger oscillatory behavior of the integral parameters related to wave profiles at Froude numbers of 0.266 and 0.313. Finally Figure 60 shows the variation of the crossflow angle across the boundary layer at three streamwise positions along the wave for $\mathrm{Fr}=0.313$. As noted earlier, these may be regarded as a measure of deflection of the free surface through the boundary layer. The three locations have been selected to show the influence of the free-surface curvature which changes from concave at $\frac{2 x}{L}=-0.750$ to cross over or S-shape at $\frac{2 x}{L}=0.380$, and all positive at $\frac{2 x}{L}=0.780$. It is seen 
that surface deflections are well correlated with curvature changes. However, the deflections are small since the Wigley hull is slender and the waves are not steep.

Figure 61 shows the comparison of the shape parameter along the first streamline for different Froude numbers. Here again the effect of Froude number on the development of the boundary layer is vividly shown by the marked oscillation in the shape-parameter distribution for $\mathrm{Fr}=0.266$ which represents the stronger wave system. The influence of the wave profile on the boundary layer lessens toward the keel. Figure 62 shows the variation of the shape parameter along the four streamlines for $\mathrm{Fr}=0.266$. Note that the shape-parameter changes are more distinct along the first two streamlines which are nearer to the free surface, and diminish toward the keel along streamlines 3 and 4. A similar trend was noticed in the results for the other Froude numbers, which, for the sake of brevity, are not shown here.

Figure 63 shows the comparison between the measured and calculated streamwise momentum thickness for $\mathrm{Fr}=0.350$. It is clear that the calculated momentum thickness is in reasonable agreement with the measurements except near the stern where the boundary-layer thickens and the thin-boundary-layer equations are no longer accurate enough. The agreement between calculated and measured values is poorer along the first streamline. This is due to the fact that the streamlines begin to converge toward the free surface after midships. This causes that boundary layer to thicken faster near the free surface and so the measured values start to deviate from the calculated results. This trend is also shown by the second streamline, but the deviation of the experimental results from calculations occurs further downstream since the rate of growth of the boundary layer along this streamline is slower than for the first streamline. Finally, along the fourth streamline near the keel, the experimental points deviate from the calculated results in the opposite direction. This can be explained by noting that, near the keel, all streamlines diverge near midships, so that the boundary layer is thicker there and then it becomes thinner toward the stern. The aforementioned is the general trend which was observed from the comparison of all the measured integral parameters with their calculated values. This is also shown in Figures 64 and 65 in which the measured shape parameter is compared with the calculated results for Froude numbers of 0.266 and 0.350 . It is evident from these 
figures that the effect of the free surface has been depicted as much weaker by the calculated results than by the measurement. This may be explained by noting that the small-crossflow approximation is in fact a two-dimensional calculation. Each streamline is treated separately without considering the effect of neighboring streamlines. A better approach, of course, would be a fully three-dimensional boundary-layer calculation. The Wigley hull, however, is a slender, or a 'weak' three-dimensional body, and the present numerical approach to calculate the development of the boundary layer seems to yield reasonable results. 


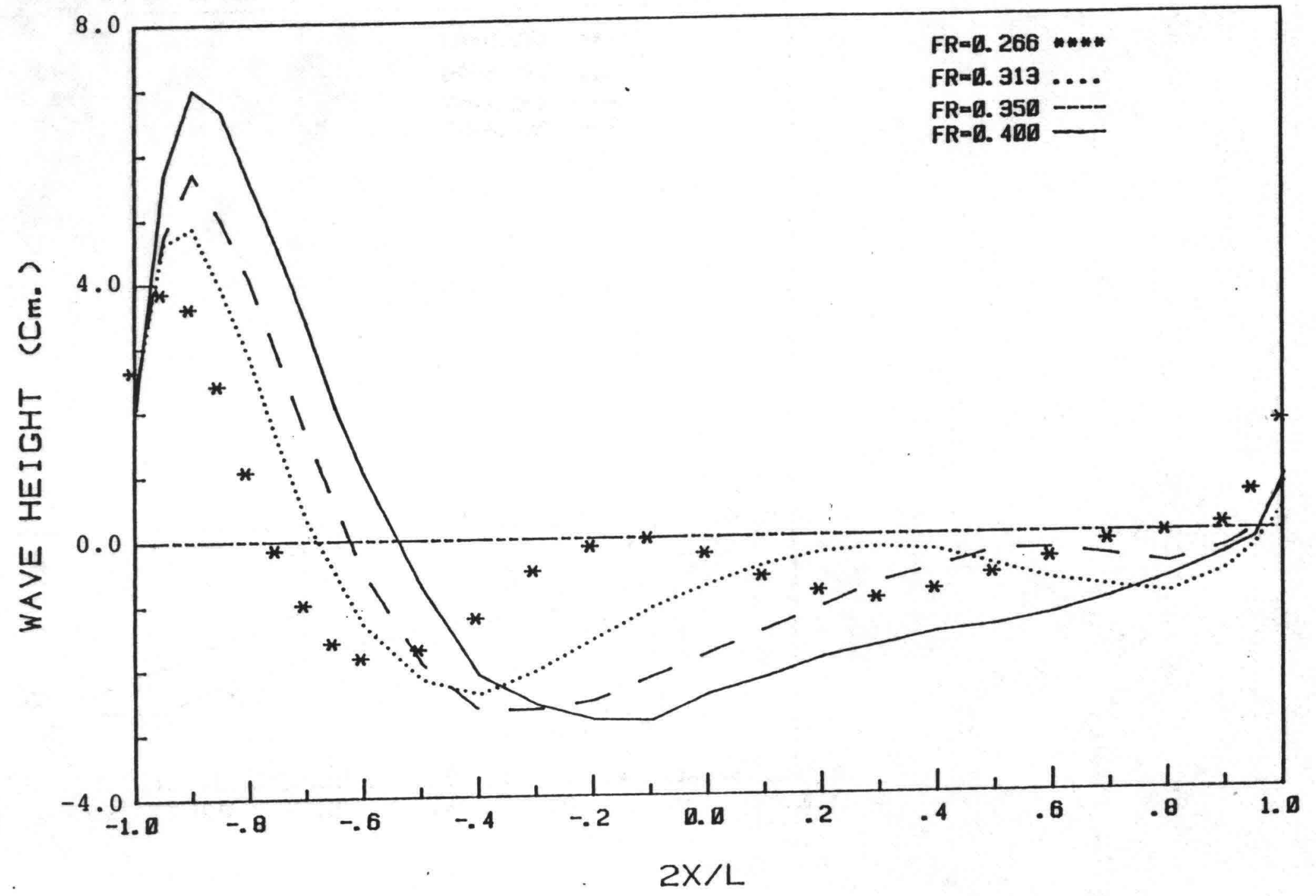

Figure 55. Measured wave profiles on the hull. 


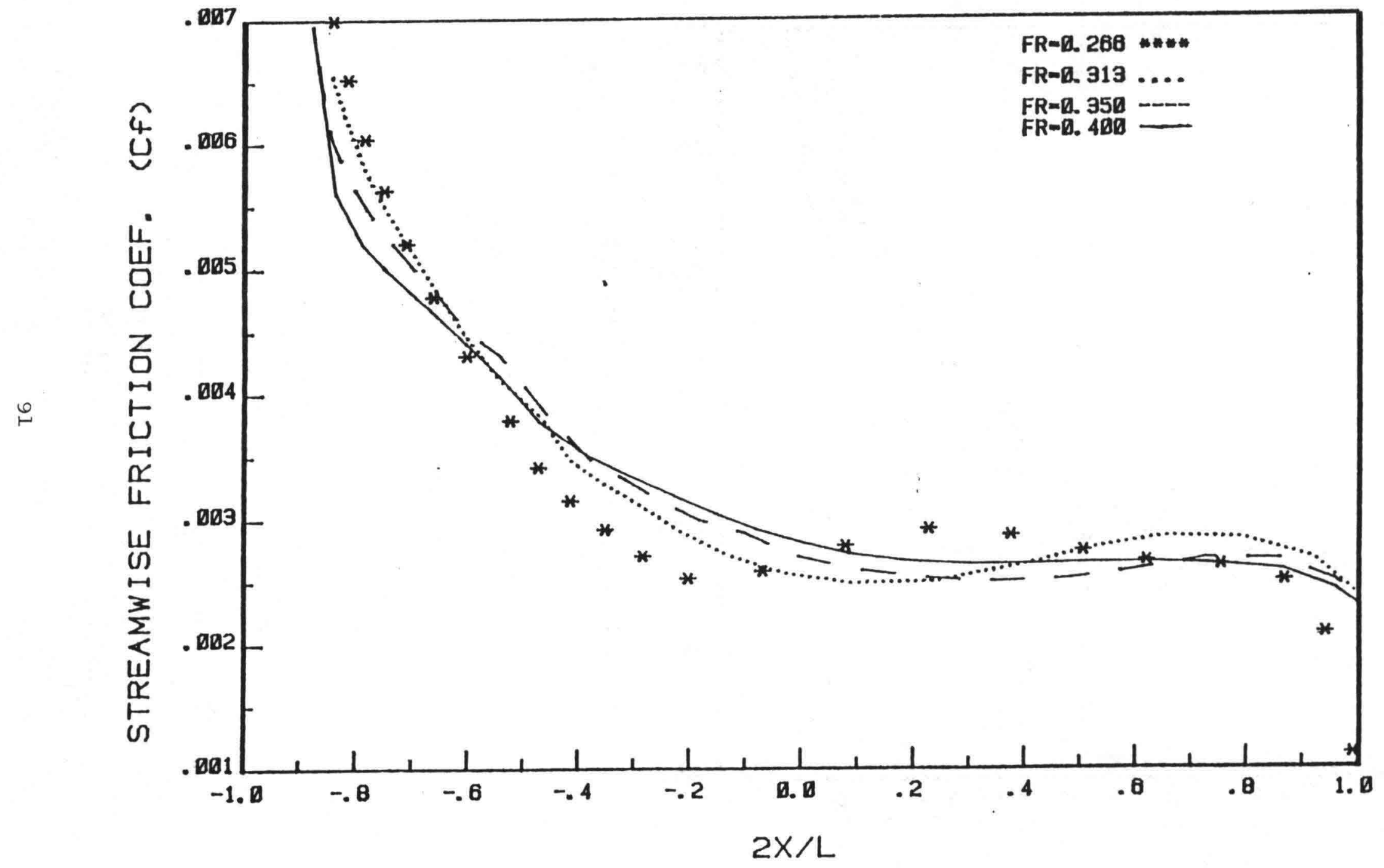

Figure 56. Skin-friction coefficient along the waves. 


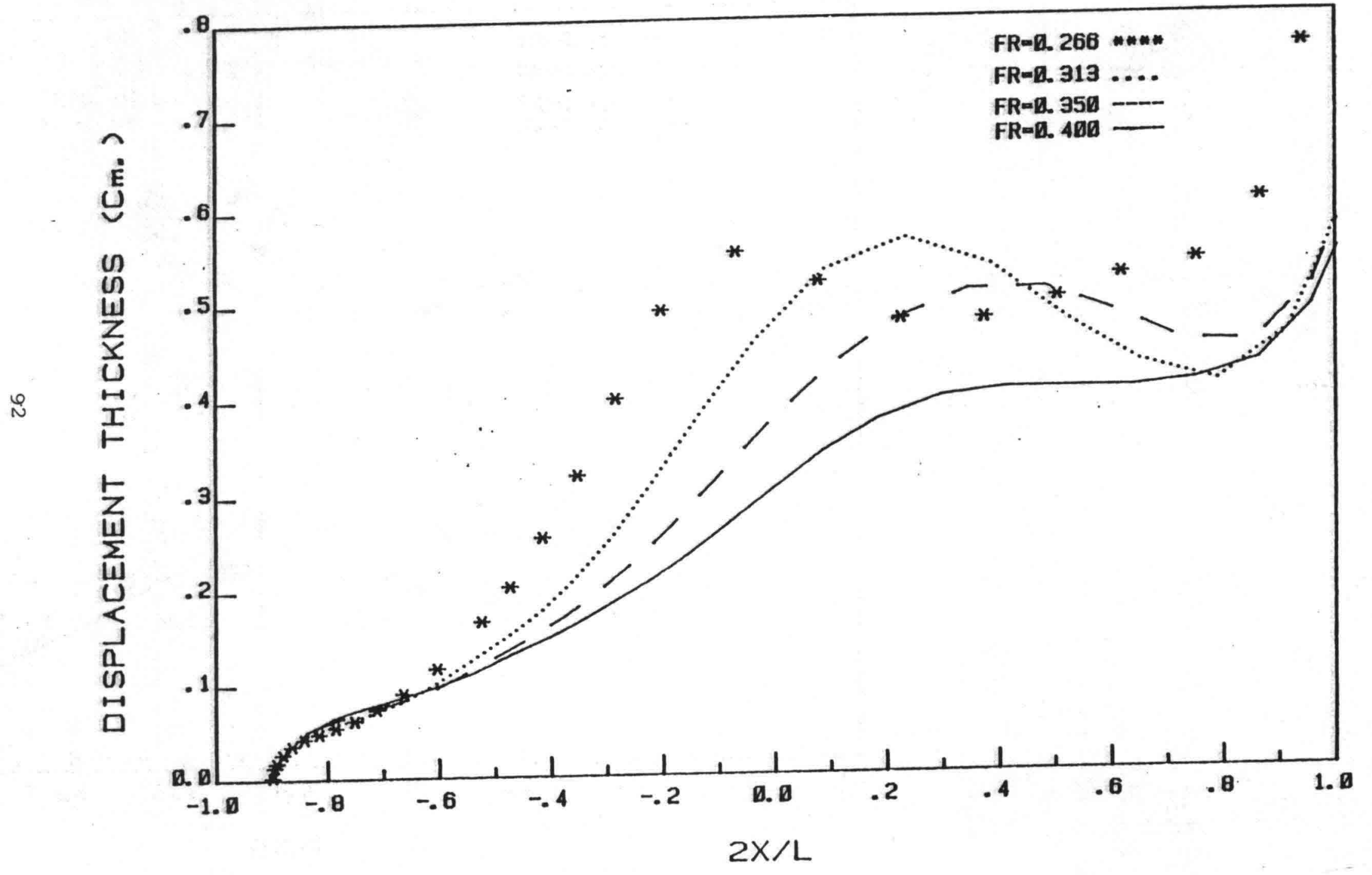

Figure 57. Displacement thickness distribution along the waves. 


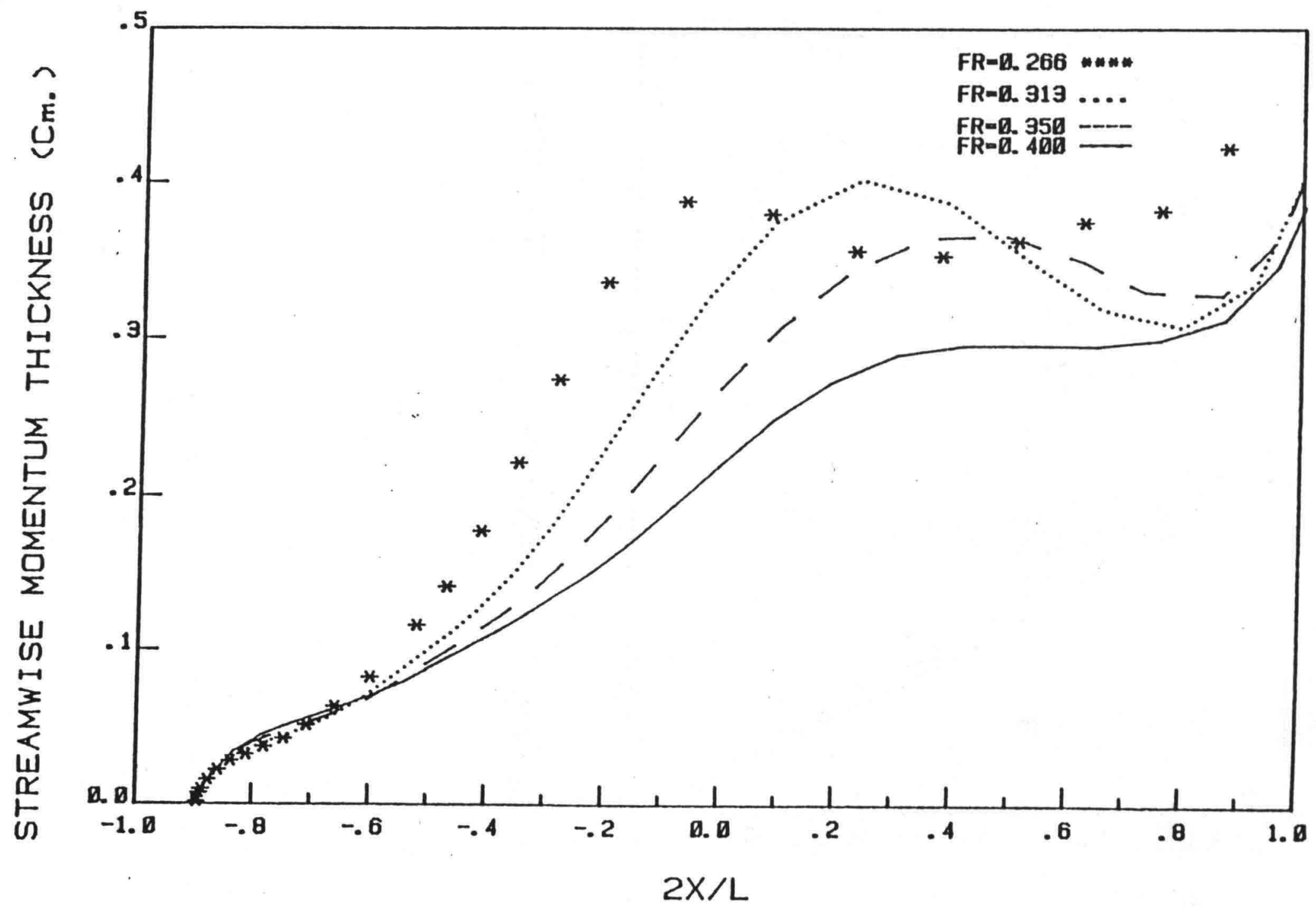

Figure 58. Momentum thickness distribution along the waves. 


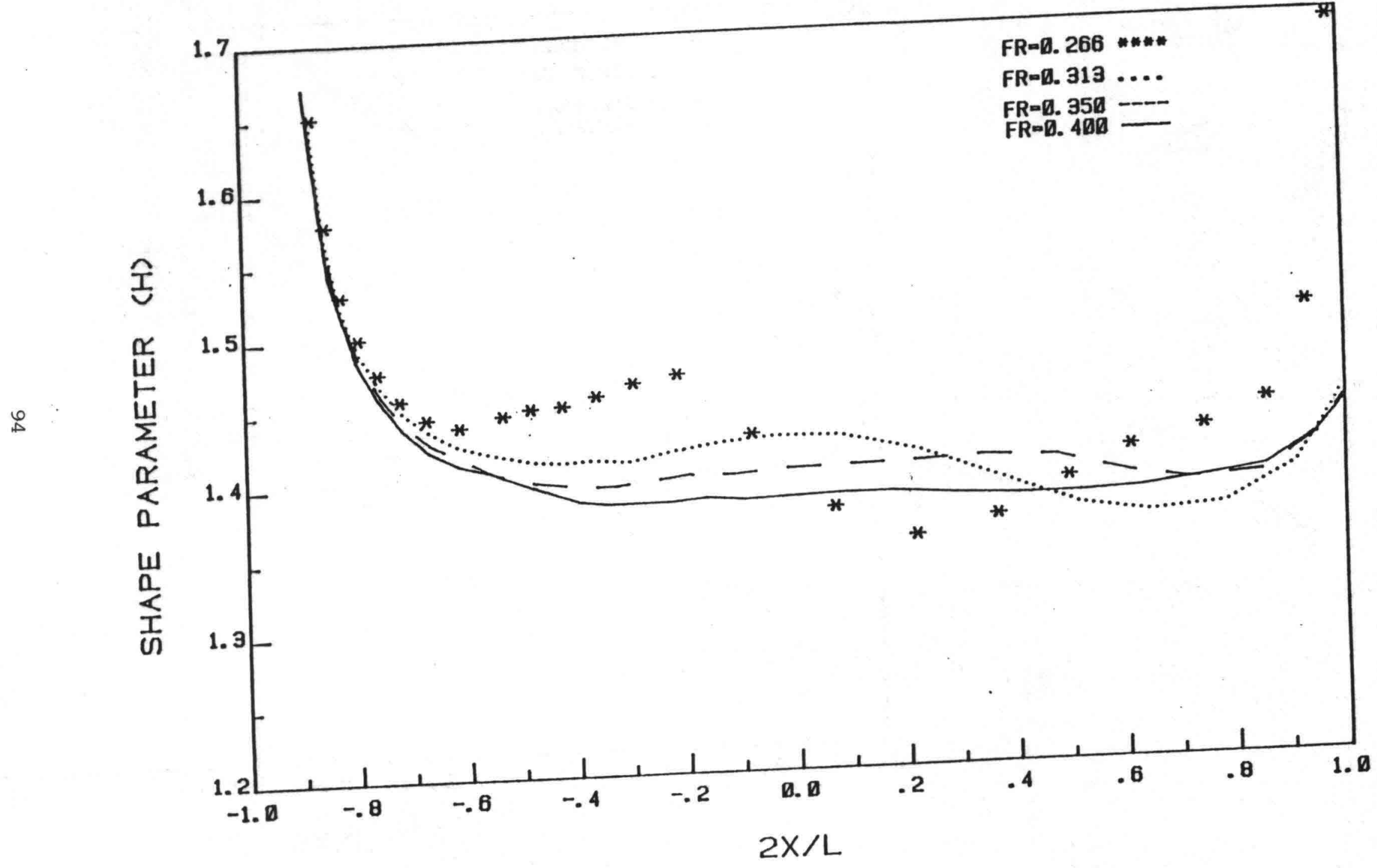

Figure 59. Shape parameter (H) distribution along the waves. 


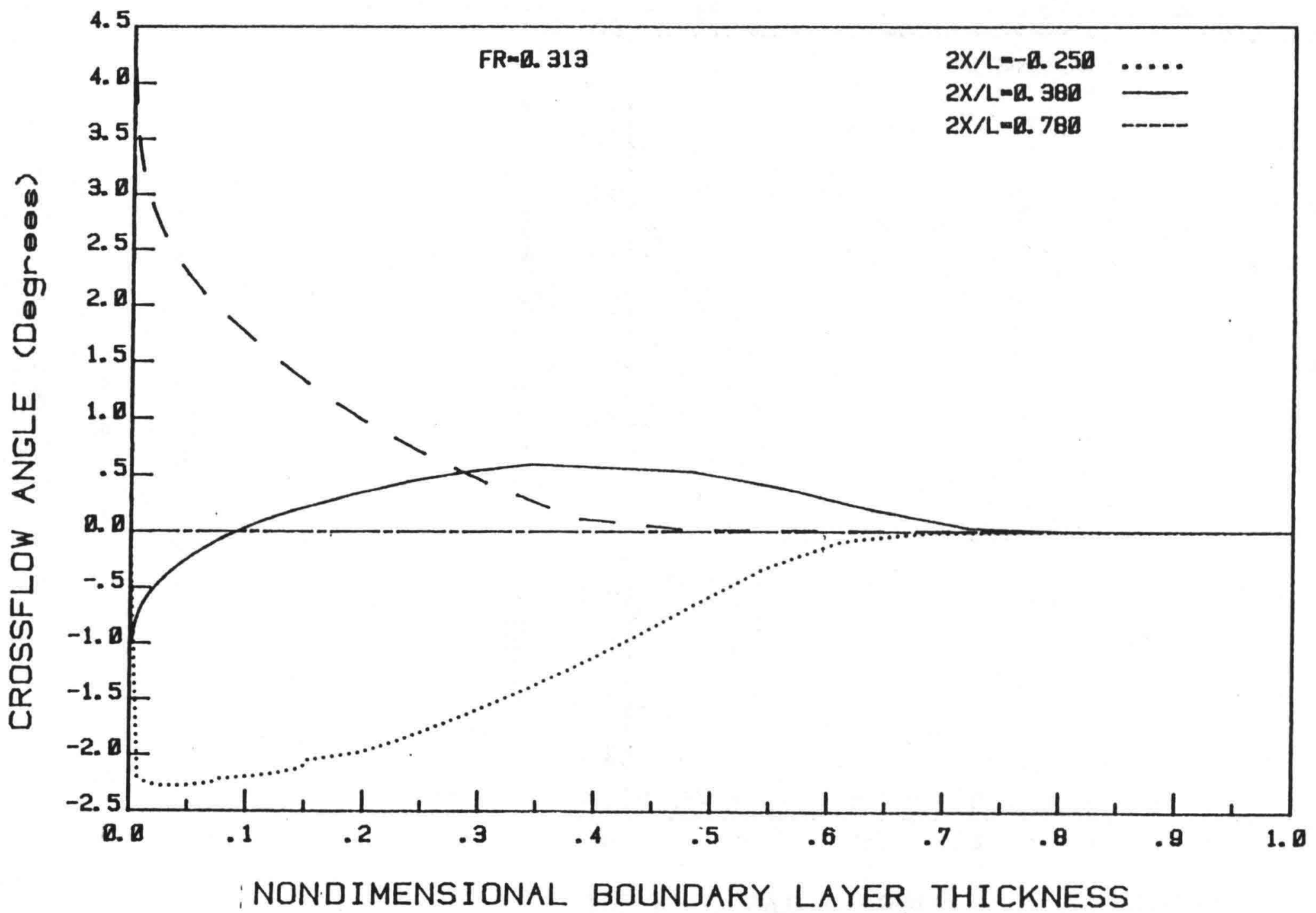

Figure 60. Variation of crossflow angle across the boundary layer $(\mathrm{Fr}=0.313)$. 


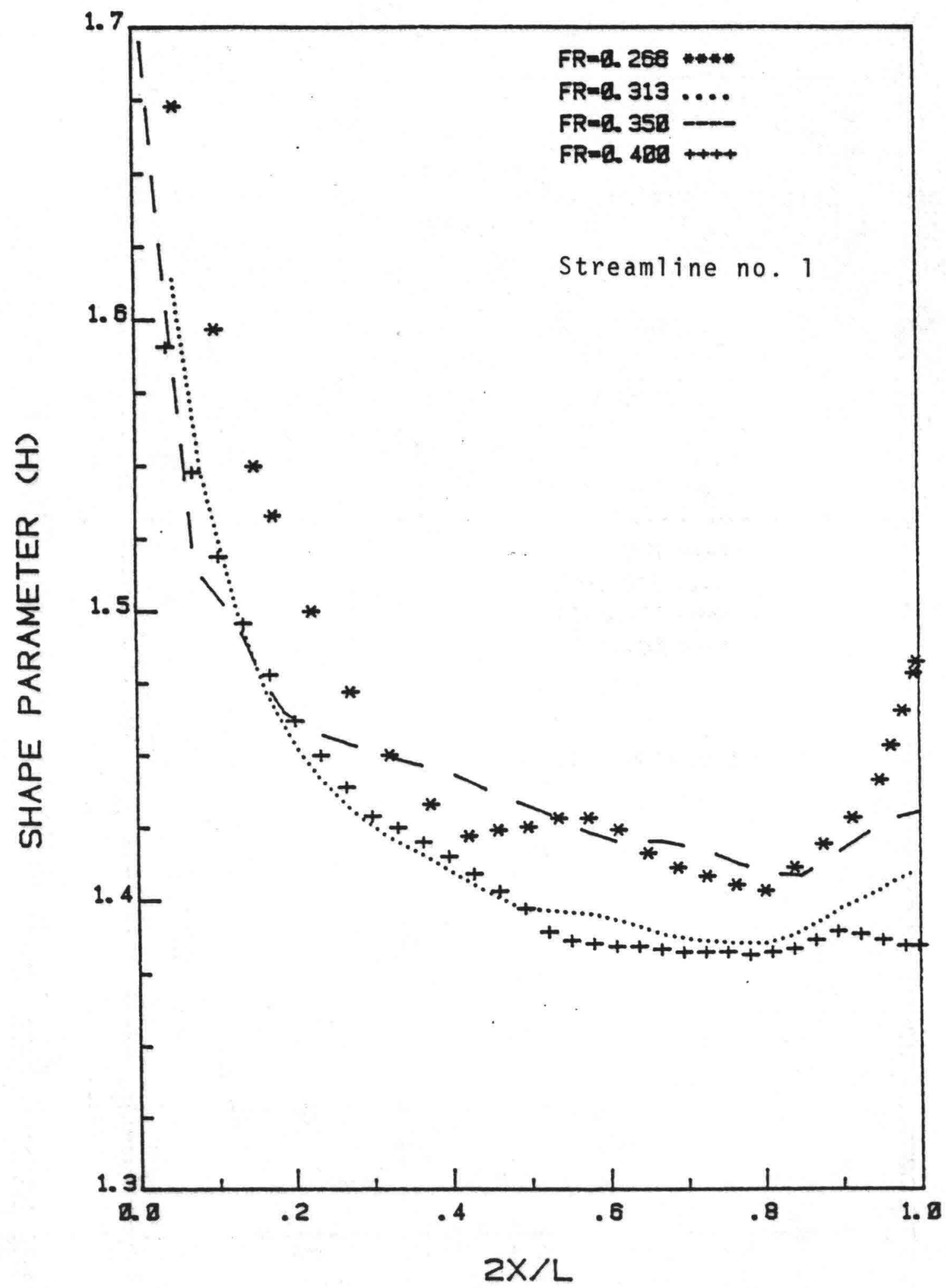

Figure 61. Variation of shape parameter along the hull for -- different Froude numbers. 


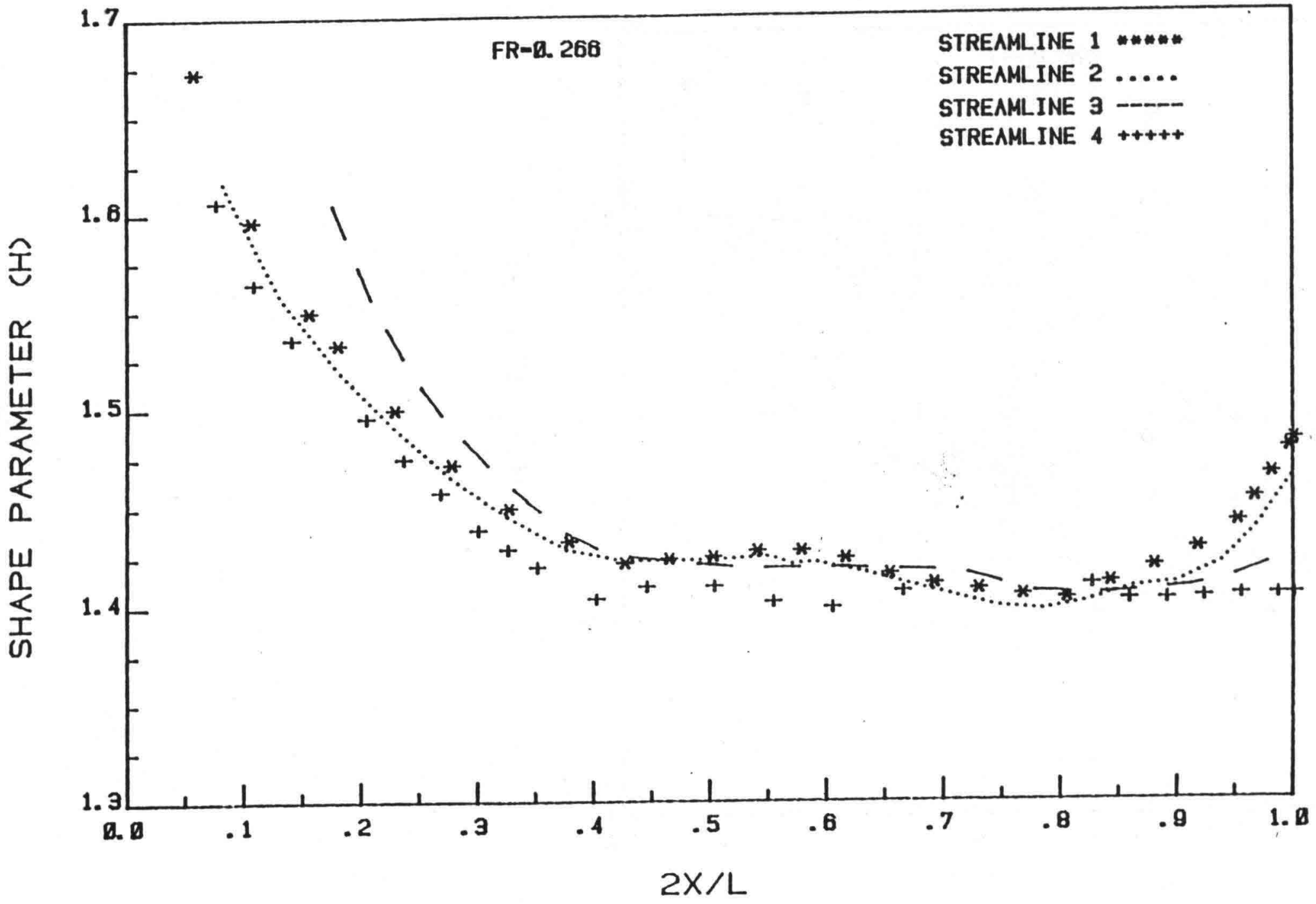

Figure 62. Variation of shape parameter along different streamlines $(\mathrm{Fr}=0.266)$. 


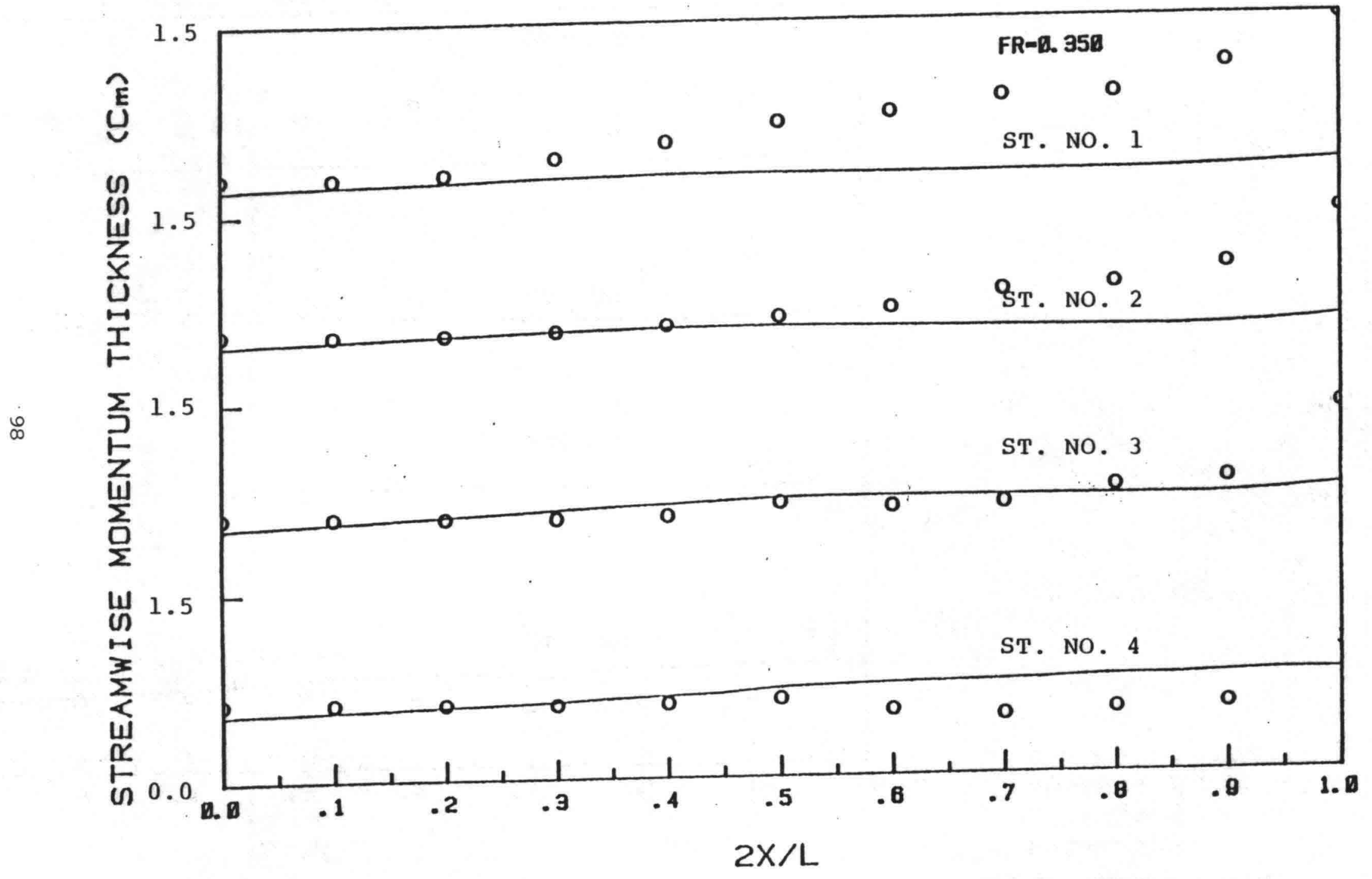

Figure 63. Comparison between calculated and measured momentum thickness $(\mathrm{Fr}=0.350)$. 


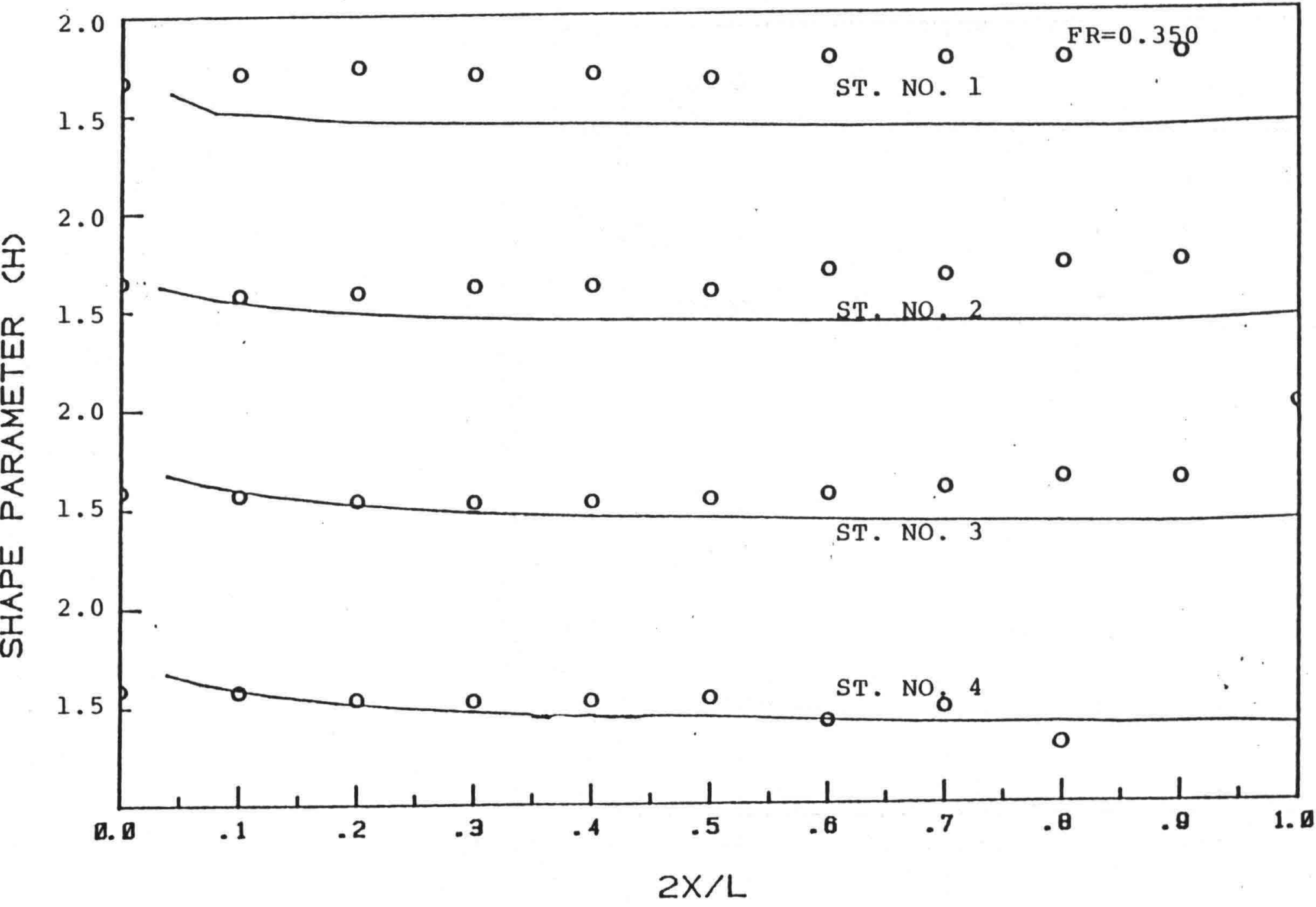

Figure 64. Comparison between calculated and measured shape parameter $(\mathrm{Fr}=0.350)$. 


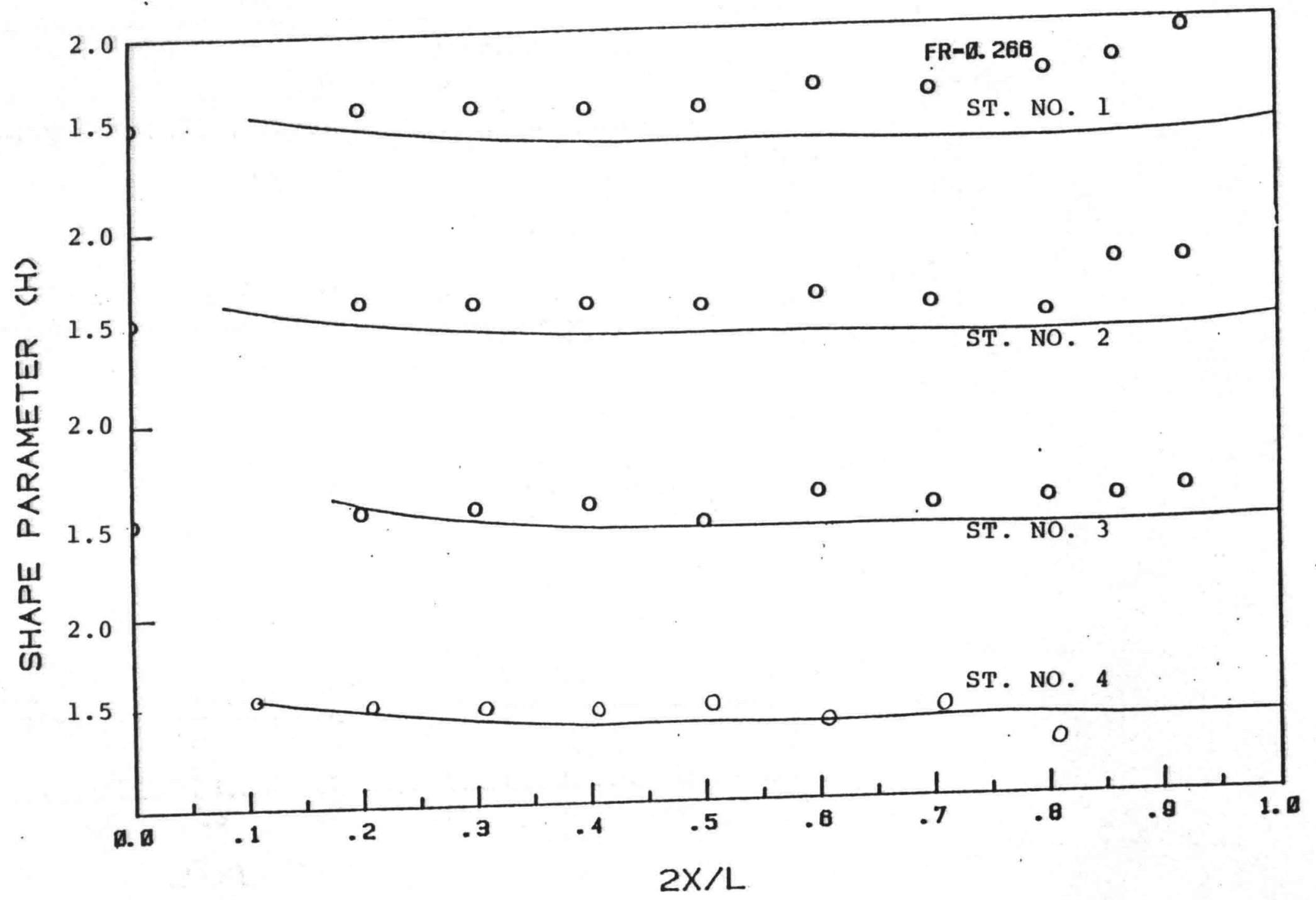

Figure 65. Comparison between calculated and measured shape parameter $(\mathrm{Fr}=0.266)$. 


\section{CHAPTER V \\ WAVE-RESISTANCE CALCULATIONS}

\section{General}

In this chapter, wave-resistance coefficients $C_{W}$ of the Wigley ship form will be calculated with three different source distributions. These distributions are: 1) Michell thin-ship distribution, 2) a first-order source distribution on the hull surface, and 3) a slender-body solution for the centerplane distribution. With the aforementioned singularity distributions, we shall consider two cases here, 1) wave resistance calculations without the viscous effects, and 2) wave resistance with the effect of viscosity take into account. All the calculations will be carried out employing the Michell integral. The residuary resistance and wave resistance obtained from towing tank tests will be used as references for comparison of different distributions.

\section{Numerical Evaluation of Michell's Integral}

\section{for the Wigley Ship Form}

The wave-resistance coefficients for the Wigley hull are given by $C_{w}$ $=R /\left(\frac{1}{2} \rho U_{0}^{2} S\right)$ where $R$ is the wave resistance. The Michell integral for a form with fore-and-aft symmetry is given by

$$
R=16 \pi \rho k^{2} \int_{0}^{\pi / 2} Q^{2}(\theta) \sec ^{3} \theta d \theta
$$

where $Q$ is the amplitude-distribution function

$$
Q=2 \int_{0}^{L / 2} \int_{-H}^{0} \sigma(x, z) \exp \left(K z \sec ^{2} \theta\right) \sin (K x \sec \theta) d x d z
$$

Here $\sigma(x, z)$ is the strength of the centerplane source distribution, $\rho$ the mass density of the fluid, $U_{0}$ the free-stream velocity, $K=g / U_{0}^{2}=\left(L F^{2}\right)^{-1}$ wher $F$ is the acceleration of gravity, $L$ is the ship length, $F_{r}$ is the Froude number, and $S$ is the wetted-surface area given by $S=0.1487 \mathrm{~L}^{2}$. 
Due to the oscillatory behavior of the integrands in (19) and (20), the control of numerical errors in the calculations is of primary concern. This has been brought out forcefully by Landweber (1979), who, in an analysis of the computed results for the wave resistance of the Wigley ship form at a workshop, showed that even the first-order calculations produced discrepancies of the order of 15 percent. In the following, a procedure in which the oscillatory part of the integrand is integrated exactly has been developed.

Consider a small element of the ship centerplane with dimensions $\Delta \mathrm{x}$ and $\Delta \mathrm{z}$ in the $\mathrm{x}$ and $\mathrm{z}$ directions, respectively. Let us

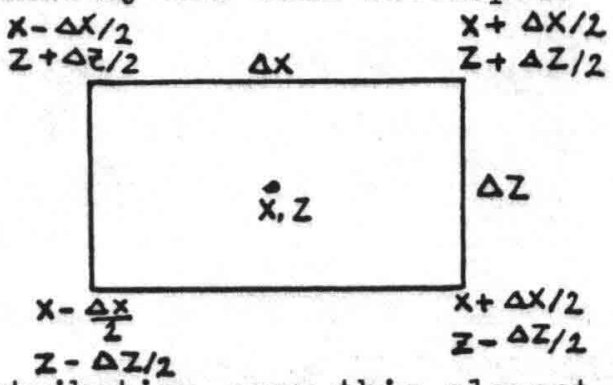
assume that the strength of the source distribution over this element can be approximated by $\bar{\sigma}_{i j}$ which is constant over the area $\Delta x \Delta z$. Thus the integral (20) for this element can be written as

$Q_{i j}=2 \bar{\sigma}_{i j} \int_{x-\Delta x / 2}^{x+\Delta x / 2} \operatorname{Sin}(K x \operatorname{Sec} \theta) d x \int_{z-\Delta z / 2}^{z+\Delta z / 2} \exp \left(K z \operatorname{Sec}^{2} \theta\right) d z$

which is exactly integrable and yields

$$
\begin{gathered}
Q_{i j}=\frac{8 \bar{\sigma}_{i j}}{K^{2} \operatorname{Sec}^{3} \theta} \operatorname{Sin}\left(K \frac{\Delta x}{2} \operatorname{Sec} \theta\right) \operatorname{Sinh}\left(K \frac{\Delta z}{2} \operatorname{Sec}^{2} \theta\right) \operatorname{Sin}\left(K x_{i} \operatorname{Sec} \theta\right) \\
\cdot \exp \left(K z_{j} \operatorname{Sec}^{2} \theta\right)
\end{gathered}
$$

After summation over the entire centerplane and substitution in (19), we obtain

$$
R=\frac{1024 \pi \rho U^{2}}{K^{2}} \int_{0}^{\pi / 2} E(\theta) \cos ^{3} \theta d \theta
$$

where

$$
\begin{aligned}
& U^{2} E(\theta)=\sin ^{2}\left(K \frac{\Delta x}{2} \operatorname{Sec} \theta\right) \sinh ^{2}\left(K \frac{\Delta z}{2} \operatorname{Sec}^{2} \theta\right) \\
& \left(\sum_{i=1}^{M_{1}} \sum_{j=1}^{M_{2}} \sigma_{i j} \operatorname{Sin}\left(K x_{i} \operatorname{Sec} \theta\right) \exp \left(K z_{j} \operatorname{Sec}^{2} \theta\right)\right)^{2}
\end{aligned}
$$


and $M_{1}$ and $M_{2}$ are numbers of step sizes in $x$ and $z$ directions, respectively. Integration by parts in (23) yields

$$
\int_{0}^{\pi / 2} E(\theta) \cos ^{3} \theta d \theta=-\frac{1}{4} \int_{0}^{\pi / 2}\left(\frac{\sin 3 \theta}{3}+3 \sin \theta\right) \frac{d E}{d \theta} d \theta
$$

The range of integration is then subdivided into $N$ equal subintervals at $0=\theta_{1}, \theta_{2}, \theta_{3}, \ldots \theta_{\mathrm{N}+1}=\frac{\pi}{2}$ and the quadrature formula

$$
\begin{aligned}
\int_{\theta_{i}}^{\theta}{ }^{i+1}\left(\frac{\sin 3 \theta}{3}+\right. & 3 \sin \theta) \frac{d E}{d \theta} d \theta \doteq \frac{1}{2}\left(\frac{\sin 3 \theta_{i+1}}{3}+3 \sin \theta_{i+1}+\right. \\
& \left.\frac{\sin 3 \theta_{i}}{3}+3 \sin \theta_{i}\right)\left(E_{i+1}-E_{i}\right)
\end{aligned}
$$

by which the rapidly varying factor $\mathrm{E}$ is treated exactly, is assumed. Here $E_{i} \equiv E\left(\theta_{i}\right)$. Substituting (26) and (25) into (23), we obtain

$$
\begin{gathered}
R=\frac{-128 \pi \rho U^{2}}{K^{2}}\left(\sum_{n=1}^{N} E_{n+1} \frac{\sin 3 \theta_{n+1}}{3}-E_{n} \frac{\sin 3 \theta_{n}}{3}+3 E_{n+1} \sin { }_{n+1}-3 E_{n} \sin \theta_{n}\right. \\
\left.\quad+E_{n+1} \frac{\sin 3 \theta_{n}}{3}+3 E_{n+1} \sin \theta_{n}-E_{n} \frac{\sin 3 \theta_{n+1}}{3}-3 E_{n} \sin \theta_{n+1}\right)
\end{gathered}
$$

Since

$$
\sum_{n=1}^{N} E_{n} \frac{\sin 3 \theta_{n+1}}{3}-E_{n} \frac{\sin 3 \theta_{n}}{3}=E_{N+1} \frac{\sin 3 \theta_{N+1}}{3}-E_{1} \sin 3 \theta_{1}=0
$$

and similarly

$$
\sum_{n=1}^{N} 3\left(E_{n+1} \sin \theta_{n+1}-E_{n} \sin \theta_{n}\right)=3\left(E_{N+1} \sin { }_{N+1}-E_{1} \sin \theta_{1}\right)=0
$$

We see that the sum of the first four terms in (27) is zero. The remaining terms then yield

$$
\begin{gathered}
C_{w}=\frac{-256 \pi L^{2}}{S} F_{r}^{4}\left[\sum_{n=1}^{N-1} E_{n+1}\left(\frac{\sin 3 \theta_{n}}{3}+3 \sin \theta_{n}\right)-E_{n}\right. \\
\left.\left(\frac{\sin 3 \theta_{n+1}}{3}+3 \sin \theta_{n+1}\right)-\frac{8}{3} E_{N}\right]
\end{gathered}
$$

where $\mathrm{E}\left(\theta_{n}\right)$ is given by (24). 
The accuracy of the integration with respect to $x, z$ and $\theta$ was investigated by halving the step size for each variable while keeping the other two fixed. By this procedure, step sizes $\Delta x=\frac{L}{100}, \quad \Delta z=\frac{H}{20} \quad$ and $\Delta \theta=\frac{\pi}{100}$ were found to give sufficiently small errors.

\section{Wave Resistance without the Effects of Boundary Layer}

In this part, the wave resistance of the Wigley form was calculated for several different source distributions, on the centerplane or on the hull, without considering the effects of the boundary layer on the wavemaking resistance. As was mentioned earlier, all the wave resistance calculations are carried out only to the first order.

\subsection{Thin-Ship Approximation}

The Michell first-order approximation for the source distribution for the Wigley form is given by

$$
\sigma(x, z)=\frac{U_{0} f x}{2 \pi}=\frac{-B x}{L \pi}\left(1-\frac{z^{2}}{H^{2}}\right)
$$

where $y=f(x, z)$ is the equation of the hull surface and $f_{x} \equiv \frac{\partial f}{\partial x}$. With this distribution, (20) is exactly integrable and the resulting equation is in much simpler form than (28); see Landweber (1979). Here (29) was treated as a general distribution and substituted into (24) and (28) for calculation of the wave-resistance coefficients. The results obtained were exactly the same as those obtained by Landweber and Celik (1980). Figure 66 shows the comparison between this calculation and the wave-resistance coefficient, $C_{w v}=C_{t}-C_{v}$, obtained from towing-tank measurements. Here $c_{t}$ is the total resistance coefficient, $C_{v}$ the viscous resistance coefficient obtained by wake survey, and $C_{W v}$ is the measured wave-resistance coefficient. This figure also shows the comparison between the calculation and residuary resistance $C_{r}=C_{t}-$ $\mathrm{C}_{\text {ITTC }}$, where $\mathrm{C}_{\text {ITTC }}$ is the International Towing Tank Committee (ITTC) 1957 friction line given by $C_{\text {ITTC }}=0.075\left(\log _{10} \text { Re }-2\right)^{-2}$.

\subsection{Hul1-Surface Distribution}

An alternative first-order source distribution on the hull surface, derived as the first approximation to the solution of the Fredholm integral equation of the second kind at zero Froude number, is given by 


$$
4 \pi \sigma(\xi, \zeta)=\frac{\mathrm{Uf}_{\xi}}{\mathrm{D}}
$$

where $\eta= \pm f(\xi, \zeta)$ is the equation of the Wigley hull surface, $D=(1$ $\left.+\mathrm{f}_{\xi}^{2}+\mathrm{f}_{\zeta}^{2}\right)^{1 / 2}$, and $\xi$ and $\zeta$ are coordinates of the points on the hull. A further improvement is given by the added-mass correction

$$
\sigma(x, z)=\frac{\left(1+k_{1}\right) U_{0} f_{x}}{4 \pi D}
$$

Here the longitudinal added-mass coefficient $k_{1}$ can be estimated from its known value for an 'equivalent ellipsoid', one having the same volume, midsection area $A_{m s}$, and free-surface area $A_{f s}$ as the original ship hull. For the Wigley ship form, $\forall=\frac{4}{9} \mathrm{BLH}, \mathrm{A}_{\mathrm{ms}}=\frac{2}{3} \mathrm{BH}$, and $A_{f s}=\frac{2}{3} \mathrm{BL}$, where $\mathrm{L}, \mathrm{H}$ and $\mathrm{B}$ are length, draft, and beam of the model, respectively. The equivalent ellipsoid with these sectional areas and volume has an axis ratio of $8: \frac{32}{15} \pi: 1$ (Miloh and Landweber, 1980), and the added-mass coefficient for this ellipsoid is $\mathrm{k}_{1}=0.0240$.

Since the source distribution in (31) is a surface distribution rather than a centerplane distribution, equations (19) and (20) need to be rederived. The wave potential for a surface distribution of sources with $\eta=f(\xi, \zeta)$ is given by: (Wehausen and Laitore, 1960)

$$
\begin{aligned}
& \phi(x, y, z)=-4 K \int_{s} \int_{0}^{\pi / 2} \sigma(\xi, \zeta)\left\{\cos \left[K(y-\eta) \sin \theta \operatorname{Sec}^{2} \theta\right]+\cos [K(y+n)\right. \\
& \left.\left.\sin \theta \operatorname{Sec}^{2} \theta\right]\right\} \operatorname{Sin}[K(x-\xi) \operatorname{Sec} \theta] \exp \left[K(z+\zeta) \operatorname{Sec}^{2} \theta\right] \operatorname{Sec}^{2} \theta d \theta d S+\phi_{2}(x, y, z)
\end{aligned}
$$

By applying the Lagally theorem, one can calculate the resistance due to this source distribution,

$$
R=-4 \pi \rho \int_{S} \sigma(x, z) \frac{\partial \phi}{\partial x} d S
$$

It should be noted that the $\phi_{2}(x, y, z)$ in $(32)$, which is a $(K, \theta)$ double integral, does not contribute to the Lagally-computed force (Lunde, 1951), and therefore has not been written in detail in (32) and will be omitted in the following calculations. 
From (32), the induced velocity can be written as

$$
\begin{gathered}
\frac{\partial \phi}{\partial x}=-8 K^{2} \int_{-L / 2}^{L / 2} \int_{-H}^{0} \int_{0}^{\pi / 2} \sigma(\xi, \zeta) \cos \left(K y \sin \theta \operatorname{Sec}^{2} \theta\right) \cos \left(K \pi \sin \theta \operatorname{Sec}^{2} \theta\right) \\
\cos [K(x-\xi) \sec \theta] \exp \left[K(z+\xi) \operatorname{Sec}^{2} \theta\right] \operatorname{Sec}^{3} \theta D(\xi, \zeta) d \xi d \zeta d \theta
\end{gathered}
$$

Here, the element of the hull surface has been projected onto the centerplane, i.e., $d S=D d \xi d \zeta$ where $D(\xi, \zeta)=\left(1+f_{\xi}^{2}+f_{\zeta}^{2}\right)^{1 / 2}$. Substituting (34) into (33) and considering the source distribution for both sides of the hull, we obtain

$$
R=64 \pi \rho K^{2} \int_{0}^{\frac{\pi}{2}} Q^{2} \operatorname{Sec}^{3} \theta d \theta
$$

where $Q$ is the amplitude-distribution function

$$
Q=\int_{-\frac{L}{2}}^{\frac{L}{2}} \int_{-H}^{0} \sigma(x, z) \operatorname{Sin}(K x \operatorname{Sec} \theta) \cos \left(K y \operatorname{Sin} \theta \operatorname{Sec}^{2} \theta\right) \exp \left(K z \sec ^{2} \theta\right) D(x, z) d x d z
$$

Here $y=f(x, z)$ is the equation of the Wigley hull.

Wave-resistance coefficients $C_{w}$ for the surface distribution (31) were calculated by using relations (35) and (36). Figure 67 shows the results of this calculation and its comparison with the Michell thin-ship approximation. The comparison shows that the results with the hull-surface distribution (30) are in poorer agreement with the residuary $C_{r}$ and measured wave resistance $C_{W v}$ than those from Michell formula (29).

\subsection{Slender-Body Solution for the Centerplane Distribution}

Landweber and $\mathrm{Ju}$ (1983), obtained a slender-body solution in terms of a centerplane source distribution for a 'modified' Wigley ship form. Their modification is that some transverse sections of the model were slightly deformed. Ship sections for $-\frac{L}{3}<x<\frac{L}{3}$ are gradually varied from a parabolic section in such a way that the midship section is transformed into an ogive with the same section area. This centerplane source distribution was used in (19) for calculation of the wave-resistance coefficients. Figure 68 shows the result of this calculation and its comparison with the Michell distribution 


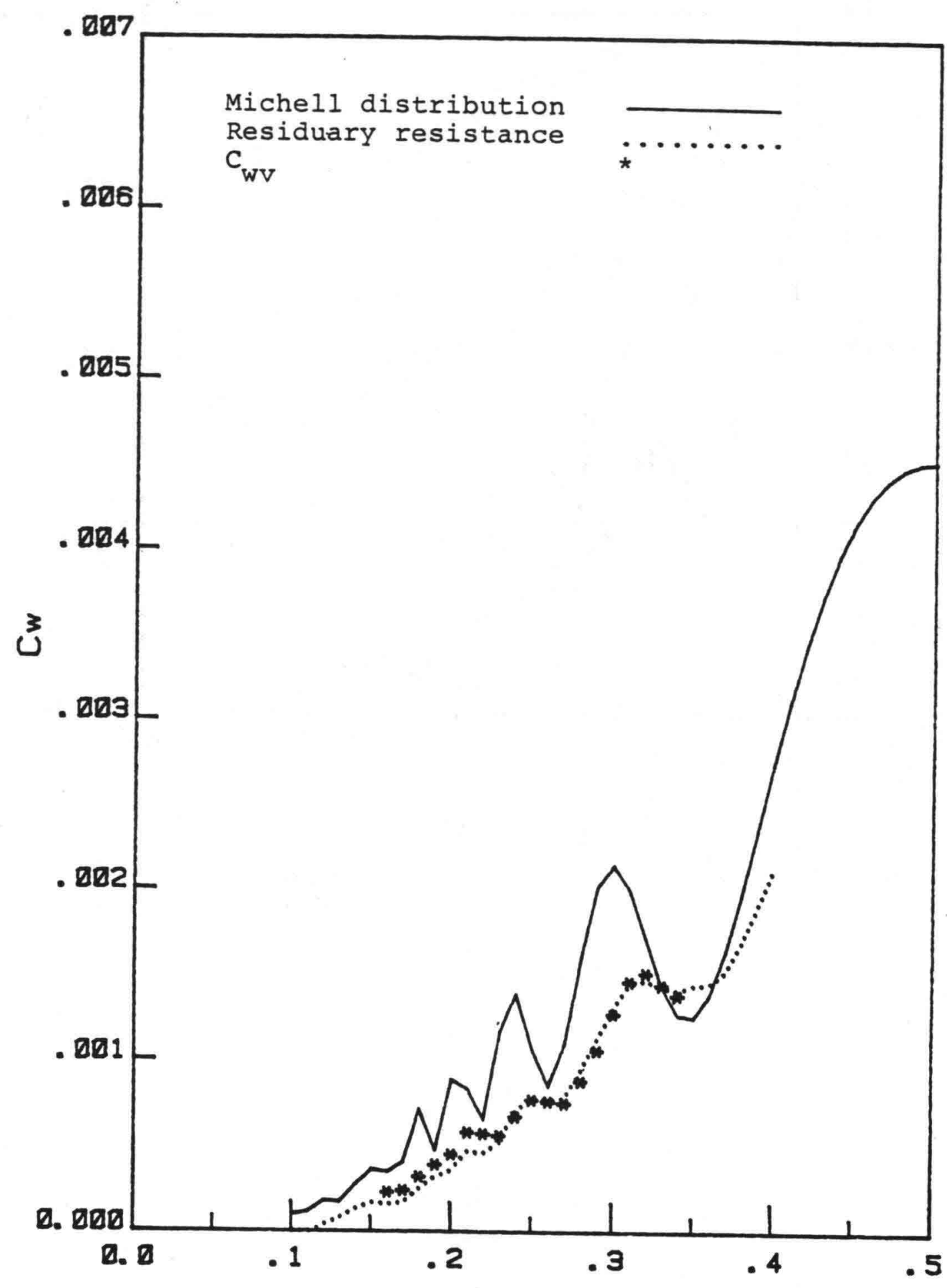

FROUDE NUMBER

Figure 66 Comparison between Michell integral and residuary resistance. 


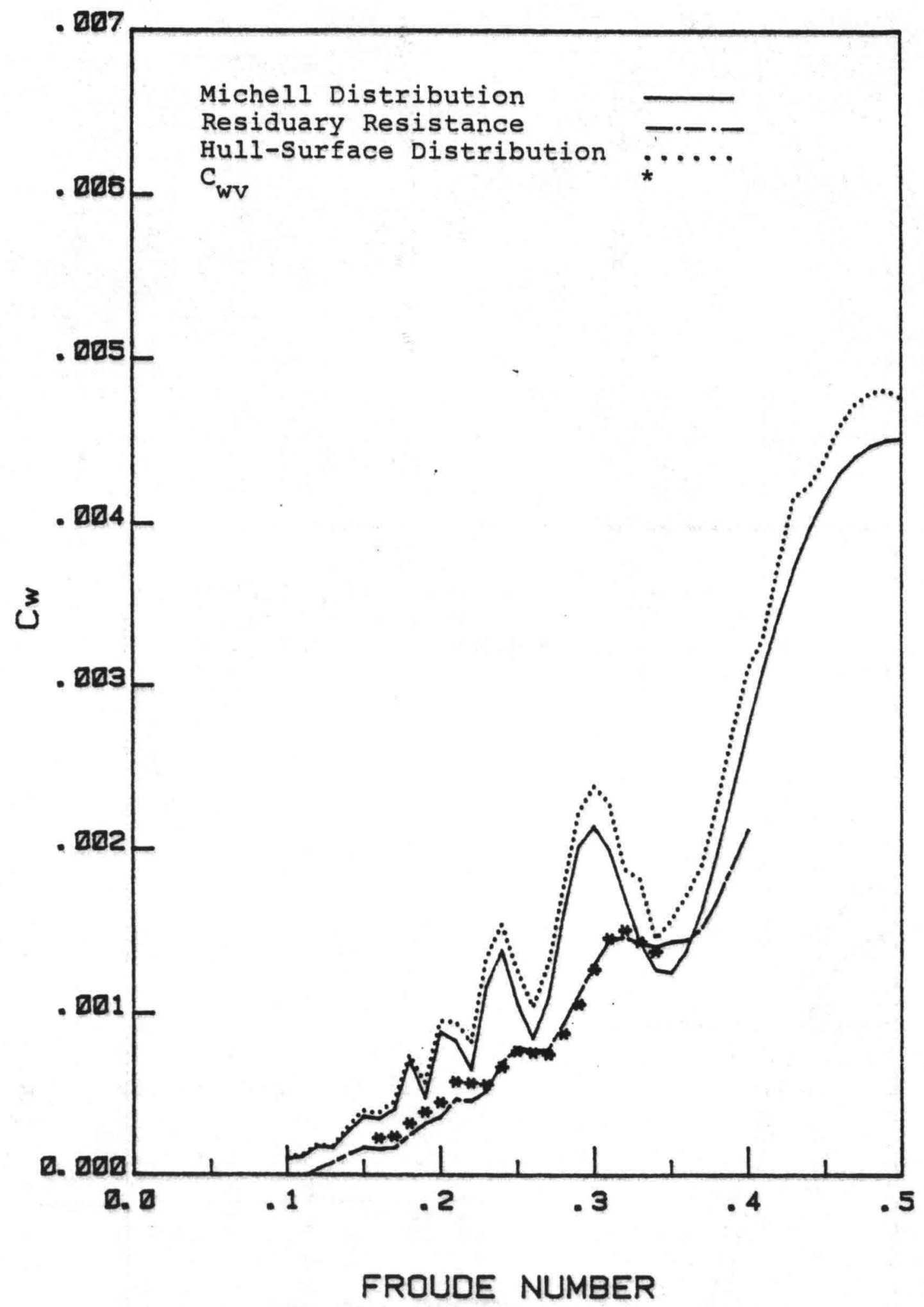

Figure 67. Comparison between hull-surface distribution and residuary resistance. 


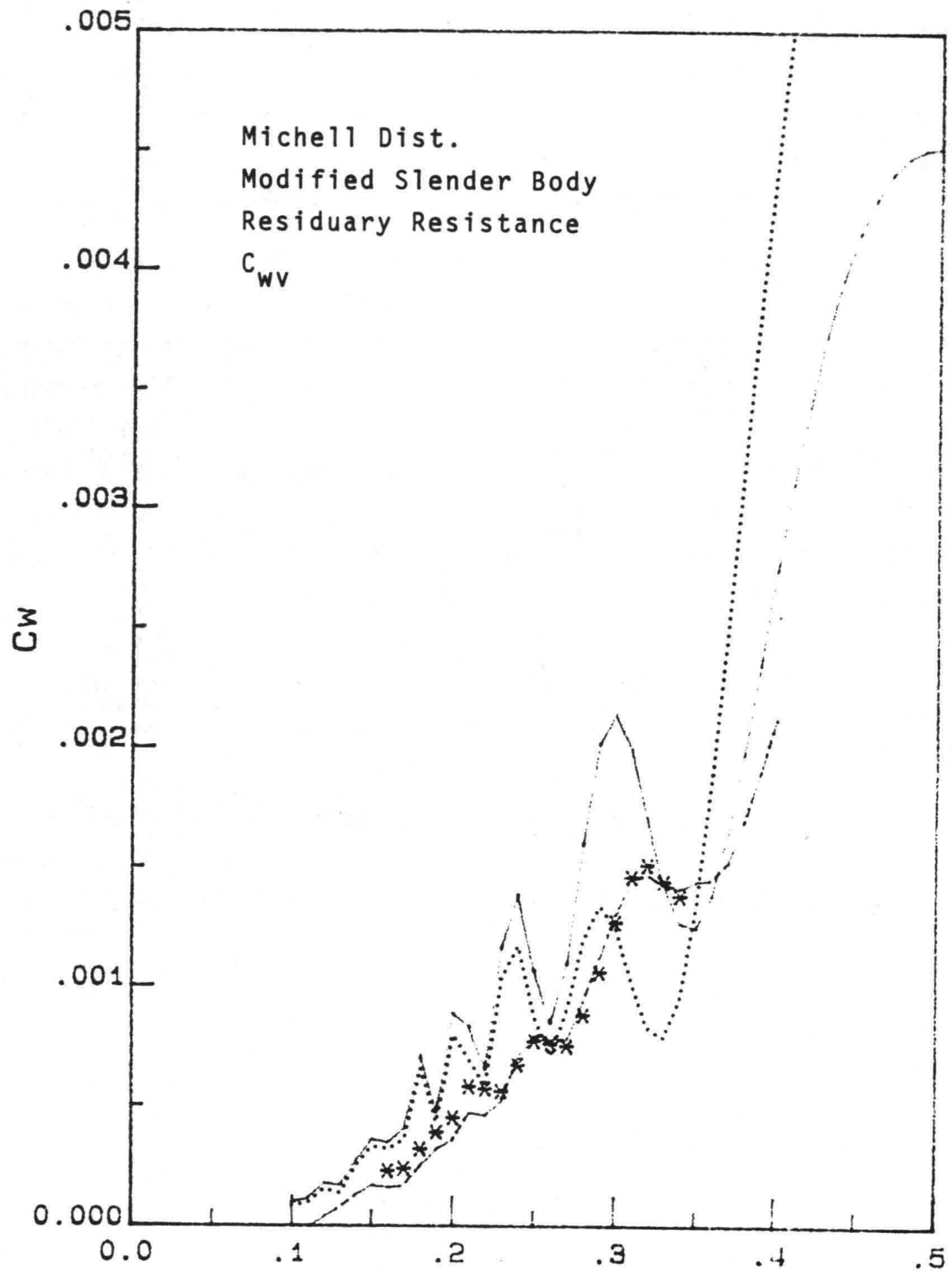

FROUDE NUMBER (FR)

Figure 68. Comparison between slender-body solution and residuary resistance. 
and the residuary resistance. The agreement between residuary resistance and this calculation is very reasonable up to about $\mathrm{Fr}=0.30$, and then deviates sharply. This may be expected since the slender-body distribution as well as the thin-ship distribution was obtained for the zero-Froude-number case and it cannot be expected to be accurate at high Froude numbers.

\section{Wave Resistance With the Effect of Boundary Layer}

The relationship between the flow exterior to a boundary layer and wake, and the concept of displacement thickness and the source distribution which generates the outer irrotational flow were examined in detail for the case of two-dimensional and axisymmetric bodies by Preston (1945), Lighthill (1958), and Landweber (1978). In continuation of his work, Landweber (1981), using an extension of the analysis used in the two-dimensional case, derived a secondorder approximation for a centerplane source distribution for a ship form. The first-order expression for the centerplane distribution of sources equivalent to the boundary layer of a ship form is given by:

$$
\sigma(x, z)=\sigma_{p}(x, z)+\frac{U_{0}}{2 \pi l_{33}}\left(l_{11} \delta_{1 x}+l_{21} \delta_{1 z}+l_{12} \delta_{2 x}+l_{22} \delta_{2 z}+K_{1} \delta_{1}+K_{2} \delta_{2}\right)
$$

where $\sigma_{p}(x, z)$ is the potential-flow distribution and the rest of the terms are due to viscous corrections. $\left\{l_{i j}\right\}$ denotes the matrix of direction cosines relating the $(x, y, z)$ coordinates of the ship form to a right-handed, curvilinear, surface-related, nonorthogonal coordinate system $(s, t, n)$, where $n$ denotes distance in the direction of the outward normal to the hull surface $S$, and $n=$ 0 on $S$. Here $s=$ constant in the zy plane and $t=$ constant in the xy plane. The unit vectors for the $(s, t, n)$ coordinate system are related to the ship hull equation $y=f(x, z)$, by

$$
\begin{aligned}
& \bar{e}_{s}=\frac{f_{x} \bar{j}+\bar{i}}{\left(1+f_{x}^{2}\right)^{1 / 2}} \\
& \bar{e}_{t}=\frac{f_{z} \bar{j}+\bar{k}}{\left(1+f_{z}^{2}\right)^{1 / 2}}
\end{aligned}
$$




$$
\bar{e}_{n}=\frac{f_{x} \bar{i}+\bar{j}-f_{z} \bar{k}}{\left(1+f_{x}^{2}+f_{z}^{2}\right)^{1 / 2}}
$$

where $(\bar{i}, \bar{j}, \bar{k})$ are unit vectors in the cartesian coordinate system $(x, y, z)$. Note that, for the $(s, t, n)$ coordinate system, $\overline{\mathrm{e}}_{\mathrm{s}} \cdot \overline{\mathrm{e}}_{\mathrm{n}}=\overline{\mathrm{e}}_{\mathrm{t}} \cdot \overline{\mathrm{e}}_{\mathrm{n}}=0$, but $e_{s} \cdot e_{t} \neq 0$. The latter product, which is a measure of the nonorthogonality of the coordinate system, obtained from (38), is

$$
\bar{e}_{t} \cdot \bar{e}_{s}=\frac{f_{x} f_{z}}{\left[\left(1+f_{x}^{2}\right)\left(1+f_{z}^{2}\right)\right]^{1 / 2}}
$$

and has its maximum $\overline{\mathrm{e}}_{t} \cdot \overline{\mathrm{e}}_{\mathrm{s}}=0$ at midship and stern, and its minimum $\overline{\mathrm{e}}_{t} \cdot \overline{\mathrm{e}}_{\mathrm{s}}=$ - 0.041 at a distance about L/3 from the midship, and $H / 2$ from the keel. $K_{1}$ and $K_{2}$ in (37) are the curvatures of surfaces $s=$ const. and $t=$ const., respectively, and since these surfaces are the $z y-$ and $x y-p l a n e s$, then $K_{1}=K_{2}$ $=0$. thickness,

Definitions of $\delta_{1}$ and $\delta_{2}$ are very similar to that of the displacement

$$
\delta_{1}=\int_{0}^{\delta} \frac{u_{e}-u}{u_{\infty}} d n, \delta_{2}=\int_{0}^{\delta} \frac{w_{e}-w}{u_{\infty}} d n
$$

where $(u, w, v)$ are the velocity components in the $(s, t, n)$ directions, and $u_{e}$, $\mathrm{w}_{\mathrm{e}}$ denote values of $\mathrm{u}, \mathrm{w}$ at $\mathrm{n}=\delta$.

As was mentioned in Chapter II, the boundary-layer measurements were originally taken in constant waterline planes. In order to calculate the various terms in (37), however, we need to have velocity components in the $(s, t, n)$ coordinate system. To solve this problem a software was developed in which first a linear interpolation between the measured velocity components on each profile was employed, and then these profiles were interpolated in the $\mathbf{x}$ and $z$ directions by least-square polynomials. The whole domain, was then divided into small cubical elements and the three velocity components were calculated from interpolating polynomials at their eight nodal points. The coordinates of points along normals to the hull were calculated from the geometry of the Wigley hull. The velocity components at each point on these normals were calculated by linear interpolation among the eight nodal points 
of the cubical element surrounding the desired point. The obtained velocity components on the normals to the hull $(\mathrm{u}, \mathrm{v}, \mathrm{w})$ were along cartesian coordinates $(x, y, z)$, respectively. The following relation was employed to transfer them to $\left(u_{s}, u_{n}, u_{t}\right)$ along $(s, n, t)$ coordinate lines:

$$
\bar{v}=u \bar{i}+v \bar{j}+w \bar{k}=u_{s} \bar{e}_{s}+u_{n} \bar{e}_{n}+u_{t} \bar{e}_{t}
$$

where $\left(\overline{\mathrm{e}}_{\mathrm{s}}, \overline{\mathrm{e}}_{\mathrm{n}}, \overline{\mathrm{e}}_{t}\right)$ are unit vectors given by $(38)$.

The complete sets of $\left(u_{s}, u_{t}, u_{n}\right)$ for Froude numbers 0.266, 0.313, 0.350, and 0.400 are stored on magnetic tapes and are available from IIHR tape library. See Appendix A.

Using these velocity profiles, the expressions (40) for $\delta_{1}$ and $\delta_{2}$ were calculated, (see Tables $5 . a$ to $5 . d$ ) and then, employing $\delta_{1}$ and $\delta_{2}$ and their derivation, the viscous-correction terms of equation (37) were calculated for all four Froude numbers and by interpolation, at several other Froude numbers. The result was a set of centerplane source distributions which extended from midship to the stern. The wave-resistance calculations for the three aforementioned cases were repeated, using expression (37) as the source distribution term in the appropriate equations. Figure 69 shows the comparison with the Michell thin-ship source distribution with and without the viscouscorrection term.

\subsection{Michell Source Distribution with Viscous Effects}

With equation (37) as the centerplane source distribution in the Michell integral, the wave-resistance coefficients for the four Froude numbers 0.266 , $0.313,0.350$, and 0.400 were calculated. Figure 70 shows the comparison between $C_{W v}, C_{r}$ and Michell centerplane distribution with and without viscosity effects. It is obvious that including the viscosity effects in waveresistance calculations modifies the theoretical resistance in the right direction, e.g., at $\mathrm{Fr}=0.313$ this lowers the curve, whereas at $\mathrm{Fr}=0.350$ this elevates the curve so that it is in much better agreement with the experimental results. 


\begin{tabular}{|c|c|c|c|c|c|c|c|c|}
\hline \multirow[b]{3}{*}{$2 x_{1}$} & \multicolumn{4}{|c|}{$\delta_{1}(\mathrm{~nm})$} & \multicolumn{4}{|c|}{$\delta_{2}(\mathrm{~mA})$} \\
\hline & \multicolumn{4}{|c|}{ Z/Hi } & \multicolumn{4}{|c|}{ ZIH } \\
\hline & 0.2 & 0.4 & 0.6 & 0.8 & 0.2 & 0.4 & 0.6 & 1.3 \\
\hline 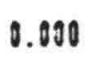 & 4.58 & 6.41 & 9.43 & 9.23 & $-\hat{0} .20$ & 0.67 & -8.65 & -9.29 \\
\hline 93 & 5.01 & 6.75 & 3.32 & $8.4 !$ & $0.2 i$ & 0.23 & 8.16 & 9.93 \\
\hline 0.400 & 9.30 & 39.36 & 19.19 & 12.24 & $-0 . j 0$ & -0.43 & .11 .23 & فُ. \\
\hline 0.560 & 11.54 & 11.48 & 40.32 & 11.95 & 0.18 & 0.36 & ב & -0.11 \\
\hline 0.600 & 13.79 & 15.33 & 10.51 & 8.40 & -4.69 & -4.36 & -0.29 & -1.54 \\
\hline 0.700 & 17.24 & 13.37 & 18.53 & 5.85 & $9.3 E$ & -0.13 & -4.37 & $-\hat{v} .3 \hat{1}$ \\
\hline 0.800 & $29: 78$ & 14.86 & 9.26 & 4.12 & -0.35 & -8.87 & -1.16 & -0.4 \\
\hline 9.366 & 24.29 & 15.36 & 7.45 & 4.55 & 0. & -0.64 & -1.47 & -8.4 \\
\hline 0.933 & 29.98 & 16.95 & 9.08 & 5.29 & 0.33 & ใ. $\partial \hat{\mathrm{c}}$ & -2.14 & -1.6 \\
\hline !.798 & 38.88 & 29.41 & 97.65 & 11.20 & -1.91 & ..9.79 & $-2.8 b$ & -1 \\
\hline
\end{tabular}

$$
\text { a. } F_{P}=0.266 \text {. }
$$

\begin{tabular}{|c|c|c|c|c|c|c|c|c|}
\hline \multirow[b]{3}{*}{$2 X / 1$} & \multicolumn{4}{|c|}{$\delta_{1}(\mathrm{~mm})$} & \multicolumn{4}{|c|}{$\delta_{2}$ (nN) } \\
\hline & \multicolumn{4}{|c|}{$2 / 4$} & \multicolumn{4}{|c|}{$2 / H$} \\
\hline & $0 . \hat{2}$ & $0 . \div$ & 0.2 & 0.3 & 0.2 & 0.4 & 0.j & 0.3 \\
\hline 0.900 & $4 . \hat{a}$ & 5.32 & 10.53 & 13.93 & -0.02 & 0.04 & -0.37 & -0.94 \\
\hline 0.200 & 5.84 & 7.33 & 10.18 & 3.59 & 0.10 & 0.14 & 0.13 & -8.15 \\
\hline 1.406 & 9.97 & 10.06 & 11.25 & 9.36 & -0.09 & -8.07 & 0.152 & -0.63 \\
\hline 1.500 & 12.76 & 11.68 & 10.30 & $\$ 0.56$ & ง. & -0.13 & 0.09 & -4.38 \\
\hline 1.600 & 15.61 & 16.66 & 11.31 & 9.58 & -3.07 & -0.23 & -3.84 & -0.37 \\
\hline 0.700 & 57.61 & 15.09 & 11.73 & 7.06 & -9.15 & -8.46 & -1.63 & -4.43 \\
\hline 8.300 & 19.62 & $15.57^{\circ}$ & 13.15 & 6.39 & $-i .21$ & -8.79 & -2.18 & -0.81 \\
\hline 1.866 & 22.41 & 17.99 & 10.50 & 5.26 & $0 . \div 2$ & -8.73 & -1.44 & -0.20 \\
\hline 1.953 & 27.34 & 18.58 & 10.41 & 6.32 & 0.94 & -0.81 & -1.48 & -0.79 \\
\hline $1.000^{\circ}$ & 37.21. & 29.98 & 19.19 & 11.46 & -3.03 & -8.60 & -2.52 & -1.85 \\
\hline
\end{tabular}

Table 5. Displacement thickness of Eq. 40. 


\begin{tabular}{|c|c|c|c|c|c|c|c|c|}
\hline \multirow[b]{3}{*}{$2 \times \Omega$} & \multirow{2}{*}{\multicolumn{4}{|c|}{$\frac{\delta_{1}(\mathrm{~m} A)}{Z \mathrm{H}}$}} & \multirow{2}{*}{\multicolumn{4}{|c|}{$\frac{\delta_{2}(\operatorname{mn})}{U / H}$}} \\
\hline & & & & & & & & \\
\hline & 0.2 & 0.4 & 0.6 & 0.8 & 0.2 & 0.4 & 0.6 & 0.3 \\
\hline 0.000 & 3.87 & 5.00 & 9.28 & 10.74 & -0.22 & -0.02 & -1.41 & 0.19 \\
\hline 8.200 & 5.62 & 5.25 & 9.61 & 10.13 & -0.12 & 0.05 & -0.11 & -4.26 \\
\hline 0.480 & 10.51 & 10.34 & 10.71 & 9.38 & -0.26 & -0.31 & 0.15 & 1.50 \\
\hline 4.508 & $\{3.74$ & $\$ 1.74$ & 10.52 & 10.53 & 1.11 & 3.25 & -0.57 & -9.28 \\
\hline 0.600 & 17.95 & 13.57 & 11.90 & 3.01 & -0.19 & -0.21 & -9.31 & -0.64 \\
\hline 0.700 & 13.49 & 15.96 & 11.27 & 7.55 & $-0.5 \mathrm{~J}$ & -0.29 & -1.88 & -0.98 \\
\hline 9.990 & 19.39 & 15.99 & $1 \hat{L} .6 \hat{J}$ & 6.55 & -1.08 & -1.49 & $-\hat{z .29}$ & $-i, 49$ \\
\hline 0.866 & 22.45 & 18.37 & 9.46 & 6.44 & -0.25 & -6.42 & -1.77 & -0.40 \\
\hline 0.953 & 26.15 & $\$ 7.53$ & 9.63 & ร. 92 & 0.04 & -1.21 & -1.71 & -3.49 \\
\hline \multirow[t]{4}{*}{1.000} & 34.86 & 29.40 & 19.01 & โ3.32 & -3.14 & 0.46 & -1.40 & -1.71 \\
\hline & \multicolumn{8}{|c|}{ c. $F_{r}=0.350$. } \\
\hline & \multicolumn{4}{|c|}{$\delta_{1}(\mathrm{man})$} & \multicolumn{4}{|c|}{$\delta_{2}(n n)$} \\
\hline & \multicolumn{4}{|c|}{ Z/H } & \multicolumn{4}{|c|}{ ZH } \\
\hline $2 \times n$ & 0.2 & 0.4 & 0.6 & 0.8 & 0.2 & 0.4 & 0.6 & 1.8 \\
\hline 0.000 & 2.57 & 3.63 & 9.48 & $\$ 0.34$ & -0.43 & $-\hat{v} .16$ & -0.33 & -0.81 \\
\hline 0.200 & 4.42 & 5.54 & 10.54 & 12.89 & -0.13 & 0.04 & -0.11 & -0.48 \\
\hline 0.400 & 10.53 & 11.01 & 9.95 & 10.94 & -0.73 & -0.10 & -0.68 & -0.16 \\
\hline 0.500 & 13.12 & 12.30 & 11.69 & 11.61 & 0.06 & -0.13 & -9.47 & -1.50 \\
\hline 8.608 & 15.97 & 14.96 & 11.85 & 8.61 & 0.12 & -0.22 & -1.79 & -1.78 \\
\hline 0.708 & 19.25 & 16.10 & 11.80 & 7.54 & -0.24 & -0.25 & -1.82 & -1.11 \\
\hline 0.808 & 20.51 & 17.26 & 11.74 & 6.19 & -0.54 & -0.94 & -2.68 & -8.73 \\
\hline 1.866 & 22.34 & 17.63 & 8.67 & 6.82 & -8.13 & $-\$ .71$ & -1.22 & -0.30 \\
\hline 1.933 & 27.41 & 18.27 & 9.29 & 5.90 & 0.21 & -8.73 & -1.05 & -1.18 \\
\hline 1.080 & 35.87 & 26.79 & 29.05 & 11.62 & -3.04 & -8.20 & -1.01 & -2.10 \\
\hline
\end{tabular}

Table 5. Continued 


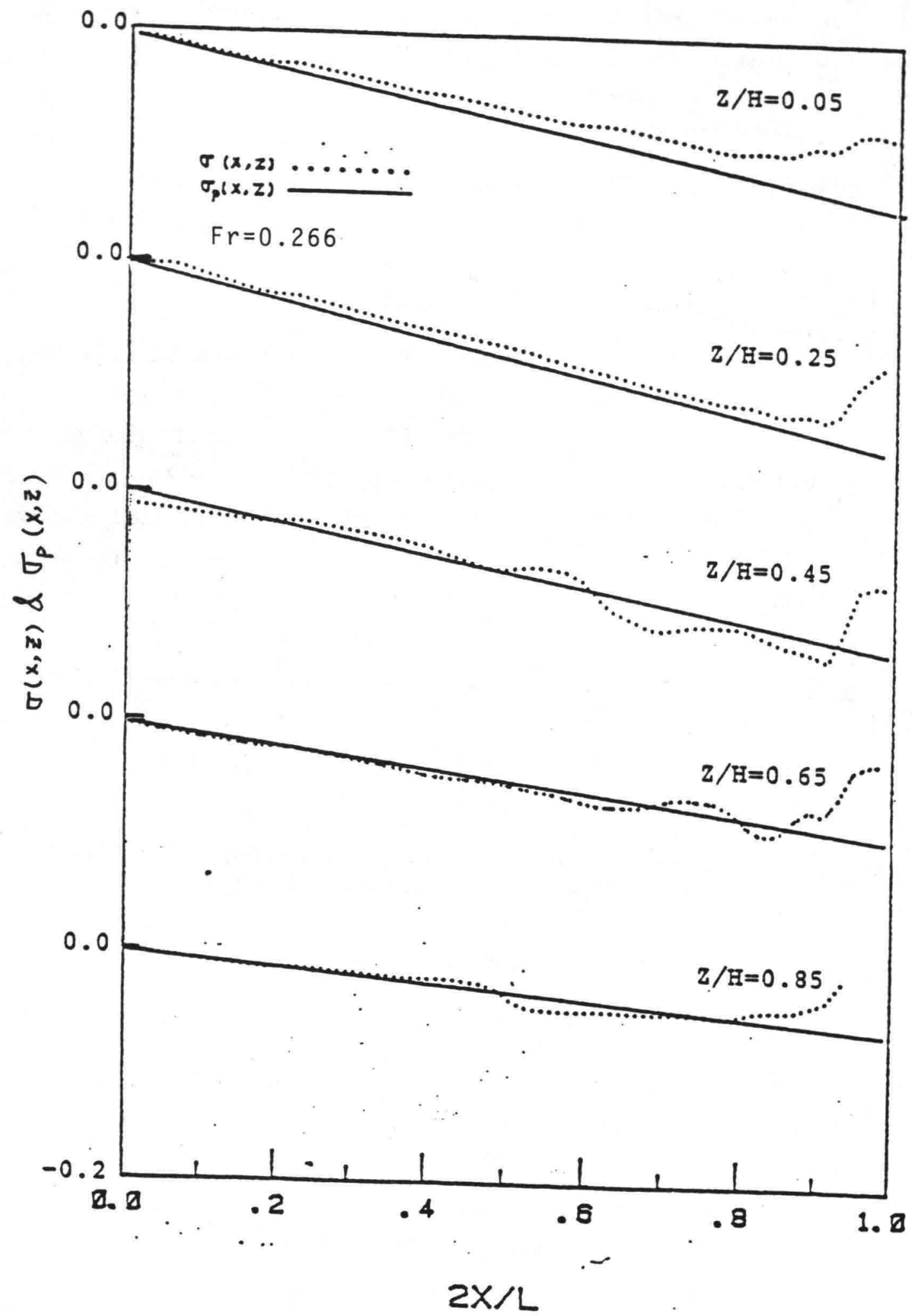

Figure 69) Comparison between $\sigma(x, z)$ and $\sigma_{p}(\dot{x}, z),(E q .37)$, for
Michell centerplane distrtbution. 


\subsection{Slender-Body Solution with Viscous Effects}

With the slender-body solution for $\sigma_{p}(x, z)$ in equation (37), the waveresistance coefficients for the modified Wigley form were calculated. Figure 71 shows the comparison between $C_{w v}$, residuary resistance, and the slenderbody solution with and without viscosity effects. Here again it is apparent that by taking effect of boundary layer into account, the theoretical results improve.

\subsection{Hull-Surface Distribution with Viscous Effects}

In order to take into consideration the effect of the boundary layer on wave resistance for hull-surface distribution, we had to modify the Michell integral. This is because the viscosity effect is in the form of a centerplane distribution of singularities while the potential flow is in the form of a hull-surface distribution. Let subscript $\mathrm{H}$ and $\mathrm{CP}$ refer to hull and centerplane, respectively. The potentials due to hull and centerplane distributions, neglecting the free-wave terms are

$$
\begin{aligned}
& \phi_{H}(x, y, z)=-8 K \int_{-L / 2}^{L / 2} \int_{-H}^{0} \int_{0}^{\pi / 2} \exp \left[K(z-\zeta) \operatorname{Sec}^{2} \theta\right] \operatorname{Sin}[K(x-\zeta) \operatorname{Sec} \theta] \\
& \operatorname{Sec}^{2} \theta \sigma_{H}(\xi, \zeta) \operatorname{Cos}\left(K y \operatorname{Sin} \theta \operatorname{Sec}^{2} \theta\right) \operatorname{Cos}\left(K n \operatorname{Sin} \theta \operatorname{Sec}^{2} \theta\right) D(\xi, \zeta) d \xi d \zeta d \theta \\
& \phi_{C P}(x, y, z)=-8 K \int_{-L / 2}^{L / 2} \int_{-H}^{0} \int_{0}^{\pi / 2} \exp \left[K(z-\zeta) \operatorname{Sec}^{2} \theta\right] \operatorname{Sin}[K(x-\xi) \operatorname{Sec} \theta] \\
& \operatorname{Sec}^{2} \theta \sigma_{C P}(\xi, \zeta) \operatorname{Cos}\left(K y \operatorname{Sin} \theta \operatorname{Sec}^{2} \theta\right) d \xi d \zeta d \theta
\end{aligned}
$$

where $y=f(x, z)$ and $n=f(\xi, \zeta)$ are equations of the hull. The Lagally theorem (33), gives two force components, $R_{1}$ and $R_{2}$, due to the surface and centerplane distributions, respectively,

$$
\begin{aligned}
& \mathrm{R}_{1}=-8 \pi \rho \int_{-\mathrm{L} / 2}^{\mathrm{L} / 2} \int_{-\mathrm{H}}^{0}\left[\left(\frac{\partial \phi_{\mathrm{H}}}{\partial \mathrm{x}}\right)_{\mathrm{H}}+\left(\frac{\partial \phi \mathrm{CP}}{\partial \mathrm{x}}\right)_{\mathrm{H}}\right] \sigma_{\mathrm{H}}(\mathrm{x}, \mathrm{z}) \mathrm{D}(\mathrm{x}, \mathrm{z}) \mathrm{dxdz} \\
& \mathrm{R}_{2}=-4 \pi \rho \int_{-\mathrm{L} / 2}^{\mathrm{L} / 2} \int_{-\mathrm{H}}^{0}\left[\left(\frac{\partial \phi_{\mathrm{H}}}{\partial \mathrm{x}}\right)_{\mathrm{CP}}+\left(\frac{\partial \phi_{\mathrm{CP}}}{\partial \mathrm{x}}\right)_{\mathrm{CP}}\right] \sigma_{\mathrm{CP}}(\mathrm{x}, \mathrm{z}) \mathrm{dx} \mathrm{dz}
\end{aligned}
$$


Differentiating (42) and (43) with respect to $\mathrm{x}$ and substituting into (44) and (45) gives the total resistance $R=R_{1}+R_{2}$,

$$
R=32 \pi \rho K^{2} \int_{0}^{\frac{K}{2}}\left(2 P+P_{0}\right)\left(P+P_{0}\right) \sec ^{3} \theta d \theta
$$

where

$$
\begin{gathered}
P(\theta)=\int_{-L / 2}^{L / 2} \int_{-H}^{0} \sigma_{H}(x, z) \exp \left(K z \operatorname{Sec}^{2} \theta\right) \operatorname{Sin}(K x \operatorname{Sec} \theta) \operatorname{Cos}\left(K y \operatorname{Sin} \theta \operatorname{Sec}^{2} \theta\right) \\
D(x, z) d x d z \\
P_{0}(\theta)=\int_{-L / 2}^{L / 2} \int_{-H}^{0} \sigma_{C P}(x, z) \exp \left(K z \operatorname{Sec}^{2} \theta\right) \operatorname{Sin}(K x \operatorname{Sec} \theta) d x d z
\end{gathered}
$$

The wave-resistance coefficients were calculated by using equation (46). Figure (72) shows the comparison among the residuary resistance coefficient, $\mathrm{C}_{\mathrm{wV}}$, and that calculated from the hull-surface distribution, with and without boundary layer effects. Here again the theoretical results are in much better agreement with residuary resistance and $C_{w v}$ when the boundary-layer effects are taken into account. 


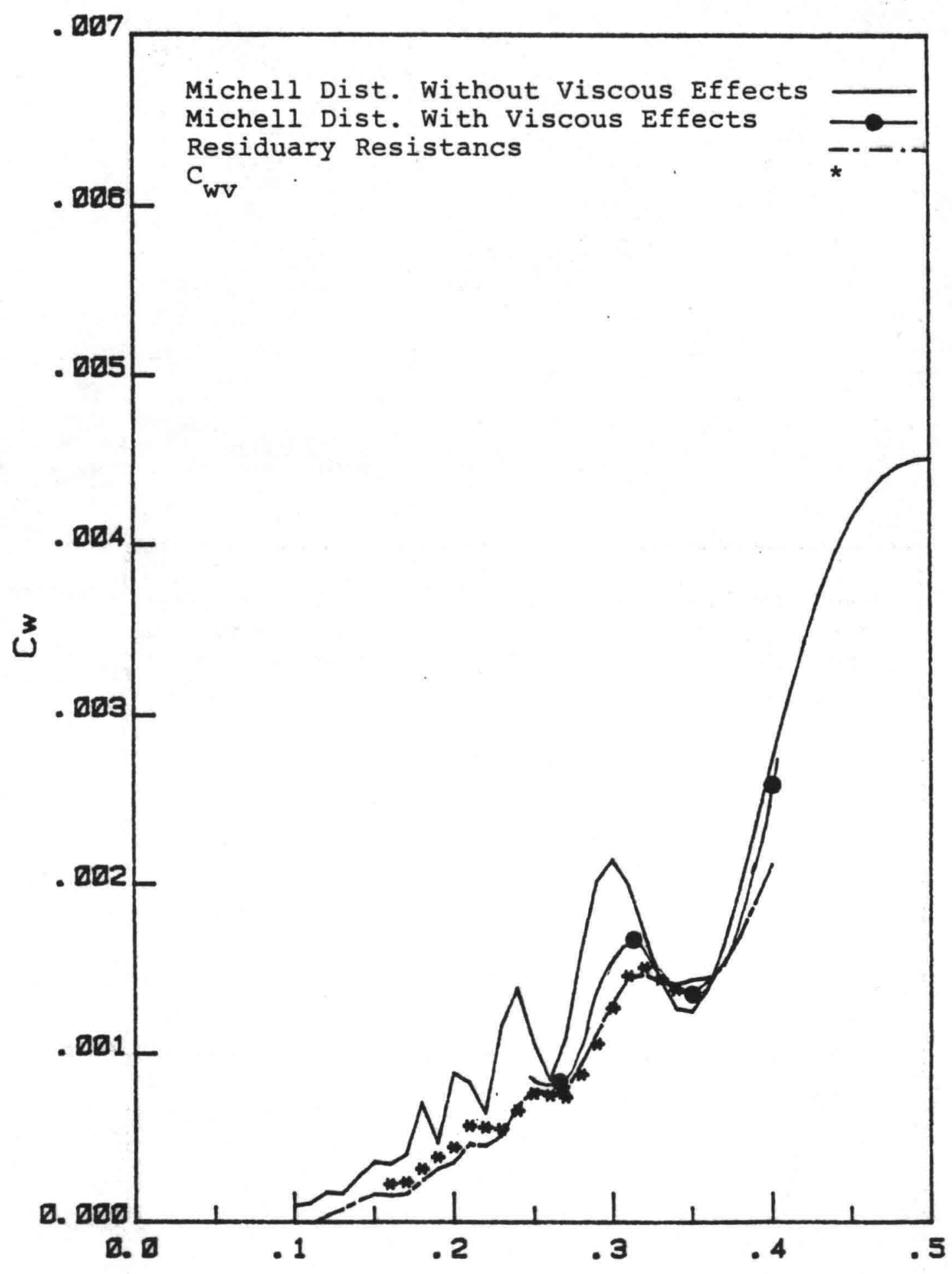

FROUDE NUMBER

Figure 70. Comparison between Michell's thin ship with and without viscous affects. 


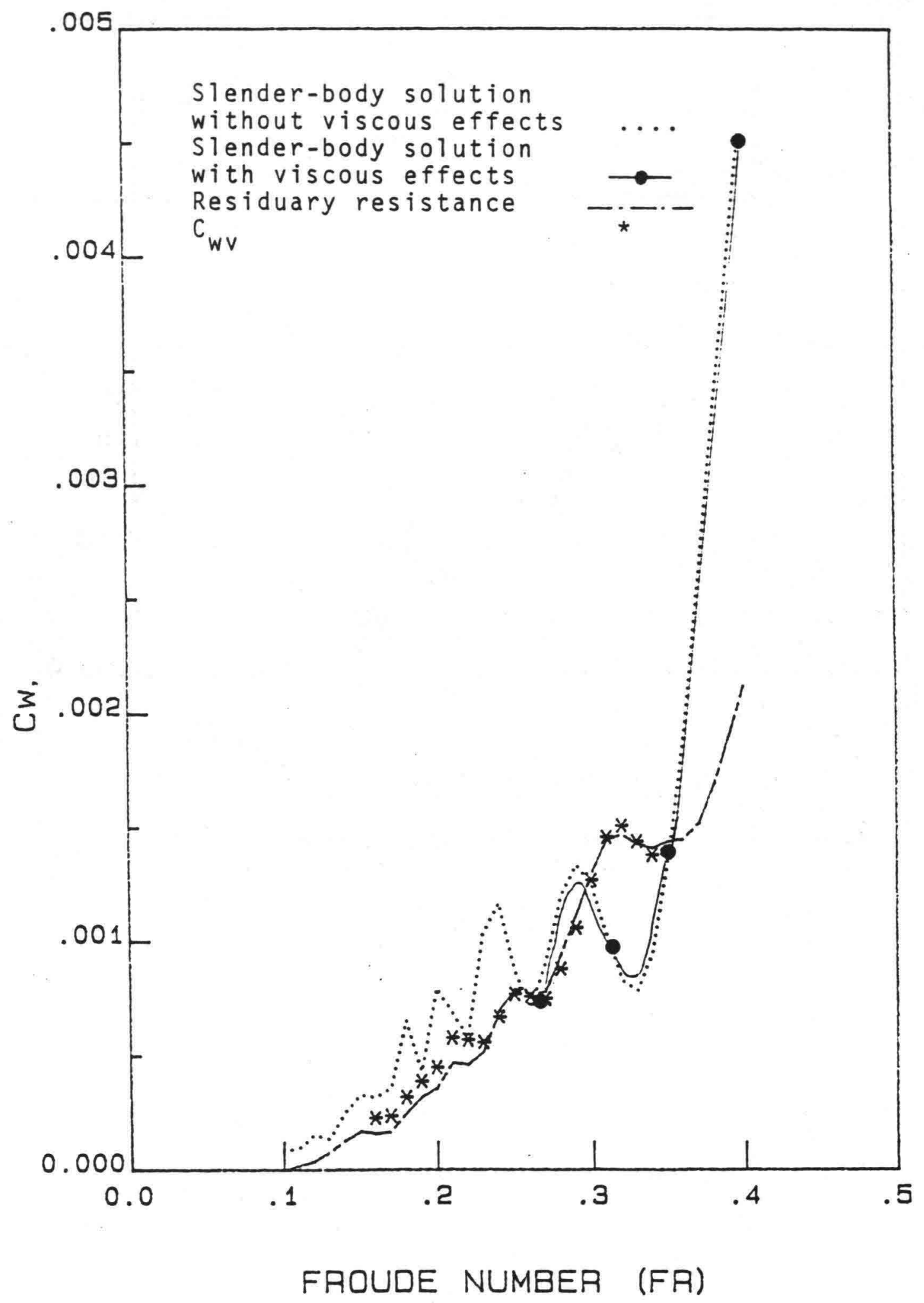

Figure $7 \uparrow$. Comparison between slender-body solutions with and without viscous effects. 


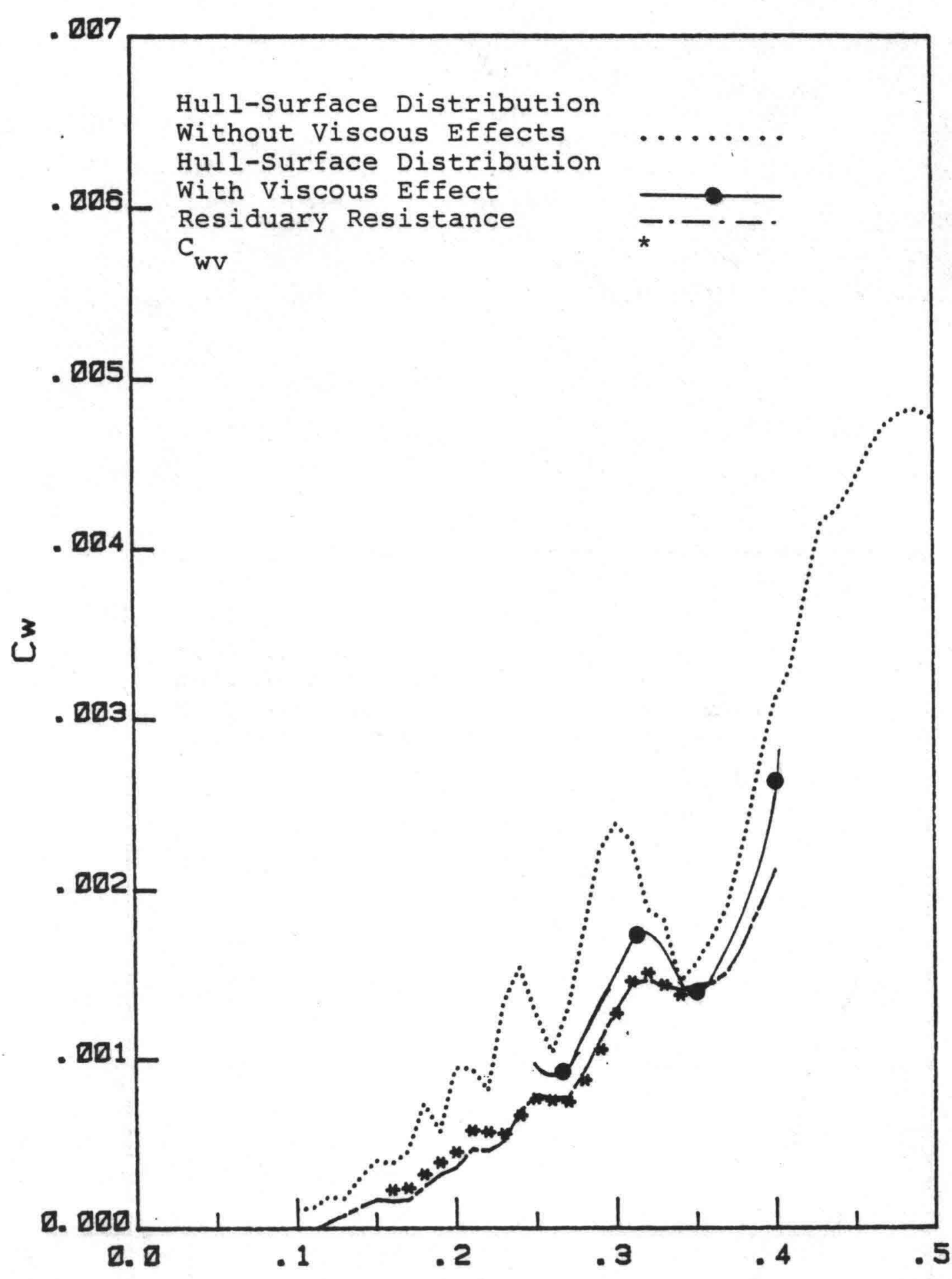

FROUDE NUMBER

Figure 72. Comparison between hull-surface distributions with and without viscous effects. 
The wave-resistance calculations for the Wigley ship form without considering the effect of viscosity showed that the Michell thin-ship theory yields better results than the first-order hull distribution. A similar result has been pointed out by Breslin (1963), and Kang (1978). It may be reasoned that the second-order terms due to the nonlinearity of the hull boundary condition and those due to the nonlinearity of the free-surface condition approximately compensate each other in the Michell integral, which gives the wave resistance from the first-order, fully-linearized theory for a thin body. In the hullsurface distribution, however, only the hull boundary condition has been improved. This indicates that all the second-order terms in the second-order thin-ship theory are quite important in wave-resistance calculations and one should consider all of them to get any better results than the Michell thinship distribution.

Table 6 shows a comparison of various results obtained in this chapter. Including the effect of viscosity in a wave-resistance calculation, even if it is limited to first-order terms only, results in a great improvement in the Michell and hull-surface-distribution calculations. It reduces the percentage of the difference between theory and experiment by more than 50\%. In the case of the modified-slender-body solution, however, including the viscous effects does not appear to change the results by much. This may be due to the fact that viscous corrections are for a Wigley hull with parabolic section and not for the modified form. Even in this case, however, introducing the viscous effects reduces the peaks of the Michell integral, resulting to a much smoother curve.

It should be noted that, in this section, contrary to Kang's (1978) analysis, we did not include the effect of wake sources on the wavemaking resistance of the Wigley hull. This is due to the fact that these sources, which represent the displacement thickness of the wake and are known as Betz (1925) sources, contribute mainly to the viscous drag of the ship model and their effect on wave resistance is negligible. It has been shown experimentally by Swain \& Landweber (1976) that the wake of a ship model would contribute only about one percent of the total wave resistance. 


\begin{tabular}{|c|c|c|c|c|c|c|}
\hline \multirow[b]{2}{*}{$F_{n}$} & \multirow[b]{2}{*}{ CHV } & \multirow[b]{2}{*}{$\mathrm{Ci}_{-}$} & \multirow[t]{2}{*}{$\begin{array}{l}\text { HITH VISLOUS } \\
\text { EFFECTS }\end{array}$} & \multicolumn{3}{|c|}{$\begin{array}{l}\text { W/O UISCOUS } \\
\text { EFFECTS }\end{array}$} \\
\hline & & & & \multicolumn{2}{|c|}{ \% Diff. } & aiff. \\
\hline & & & \multicolumn{4}{|c|}{ MICHELL DISTRIBUTION } \\
\hline 0.26o & 0.19075 & 9.30077 & .000836 & $8.5 \%$ & .000772 & $26 . \%$ \\
\hline 0.313 & ก. $991 \div 9$ & 0.00146 & .821674 & $14.6 \%$ & .001912 & $30.9 \%$ \\
\hline 0.359 & - & $0.001+4$ & .001352 & $6.1 \%$ & .001249 & $13.2 \%$ \\
\hline \multirow[t]{2}{*}{0480} & - & 0.80212 & .302590 & $22.1 \%$ & .002737 & $29.1 \%$ \\
\hline & & & \multicolumn{4}{|c|}{ HULL-SURFACE DISTRIBUTION } \\
\hline 9.265 & 0.30775 & 0.09897 & .010900 & $16.8 \%$ & .001178 & $51.9 \%$ \\
\hline 0.313 & 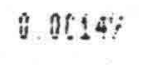 & 0.09190 & 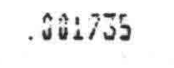 & $18 . \dot{8} \%$ & .002168 & $48.5 \%$ \\
\hline 1. $35 !$ & - & 0.03144 & .001339 & $2.3 \%$ & .001570 & $9.0 \%$ \\
\hline \multirow[t]{2}{*}{0.410} & - & $0.09 D^{2} \leq 2$ & .0012605 & $24.3 \%$ & .003105 & $46.0 \%$ \\
\hline & & & \multicolumn{4}{|c|}{ SLENDER-GODY SOLUTION } \\
\hline ל & 8.90975 & จ. ติบง77 & .000737 & 4.2\% & .000825 & $7.1 \%$ \\
\hline 0.313 & 1). $31914 ?$ & 0.60140 & .000775 & $33.0 \%$ & .000960 & $34.0 \%$ \\
\hline 0.353 & - & 0.00144 & .001393 & $3.2 \%$ & .001341 & $6.8 \%$ \\
\hline 0.430 & - & 0.09212 & .004507 & $112 . \%$ & .004572 & 115.7 \\
\hline
\end{tabular}

Table 6. Comparison between different methods of wave
resistance cal.culation with and without viscosity
effects. 


\section{CHAPTER VI}

\section{CONCLUSIONS AND RECOMMENDATIONS}

The most useful purpose served by the present study is the collection of a complete set of data on boundary-layer, pressure-distribution, and resistance measurements for the Wigley parabolic hull. These data, to the best of our knowledge, are the first complete set of boundary-layer measurements which have been obtained for a ship model at zero trim and sinkage. This is very significant, because most computation methods are made for the fixed-model condition. For example, if one cannot predict wave resistance for the fixedmodel condition, a good prediction for the more realistic free-to-trim-andsink condition is unlikely, since one has to use the fixed-model condition as the initial condition of an iterative procedure. In short, the data obtained in the present study can serve as the basis for further theoretical studies of the Wigley hull. The major conclusions of this study are as follows:

1) Experimental results for the total-resistance coefficients of the Wigley parabolic hull for free and fixed conditions are shown in Tables 1 and 2 , respectively. The comparison between these two tables shows that the hump and hollows for both cases occur at the same Froude numbers, but the values with the free condition are greater than those with the fixed condition with the increment increasing to about ten percent at the largest Froude number of 0.400 .

2) Experimental results for the pressure-distribution coefficients $C_{p}$ of the Wigley hull, restrained in both trim and sinkage, are shown in Table 3. An important feature of these measurements is the fact that the pressure distribution on the hull closely follows the trend of the wave profile at the free surface, i.e., shows the same hollows and humps. This shows that the Froude number plays an important role on the boundary layer and its development on ship hulls.

3) Three-dimensional boundary-layer measurements were carried out for four Froude numbers of $0.266,0.313,0.350$ and 0.400 . The three components of the velocity vector were obtained at ten transverse sections and five water- 
lines at each section, from midship to the stern. These measurements were extended into the near wake of the model up to a distance of 0.1 model lengths downstream from the stern. The wake measurements were carried out at four sections and five waterlines at each section. The results of measurement are given in Appendix B.

The boundary-layer and wake measurements show no flow reversal or separation for the Wigley hull. This indicates that this model is a very good research vehicle for comparison of the analytical results or numerical procedures with experimental data.

4) The boundary-layer measurements showed that the flow over the Wigley hull may be regarded as one of small crossflow, since the measured crossflow angles are less than ten degrees.

5) The original measurements, which were taken in transverse sections and along constant waterlines, were transferred by interpolation to data along normals to the hull.

6) Boundary-layer calculations on the Wigley hull were performed using the small crossflow approximation of the boundary-layer equations. These calculations were carried out along the streamlines on the hull obtained using Guilloton's method.

The comparison of the calculated and measured results show good agreement over most of the hull except near the stern where the boundary layer is rather thick.

7) Wave-resistance coefficients of the Wigley hull were calculated by using the Michell integral. These calculations were carried out by employing the Michell thin-ship centerplane distribution, a first-order hull-surface distribution, and a slender-body source distribution. Comparison of the results showed that the Michell thin-ship distribution gives a better estimate of the wave-resistance coefficients than the hull-surface distribution, but the gold medal should go to the slender-body solution which gives the best comparison with the measured wave resistance up to $\mathrm{Fr}=0.3$. The agreement between experimental results and all the aforementioned calculations becomes poorer with increasing Froude number. This is due to the fact that the calculations are based on linearized theory and zero-Froude-number distributions and cannot be expected to yield satisfactory results at high Froude numbers. 
8) The equivalent irrotational flow exterior to the boundary layer was obtained by generating the potential flow exterior to the boundary layer by means of a centerplane source distribution which satisfies the boundary condition on the edge of the boundary layer.

9) Using the equivalent irrotational flow, the wave-resistance coefficients of the Wigley ship form were calculated in combination with the distributions mentioned in (7) to study the effect of viscosity on the wavemaking resistance of the ship model. These calculations showed that the agreement with the "measured" resistances was considerably improved by modifying the source strength for the effects of viscosity.

10) The comparison between different methods of calculation showed that introducing the corrections for viscous effects improved the results with the Michell thin-ship and the hull-surface distribution more than with the slender-body solution although the latter needed only a small correction.

All the wave-resistance calculations in the present study were limited to first-order terms. For future work, it is recommended that second and higherorder terms be taken into account. The slender-body solution, in particular, may be the best answer.

Although the effects of viscosity, in the present study, were confined only to the boundary layer over half of the ship model, the results showed marked improvement. A better agreement between theory and experiments may be obtained if one considers the boundary layer and wake of the whole model, and since boundary-layer measurements in a towing tank are formidable task, the next best alternative would be to calculate the ship boundary layers numerically, for nonzero Froude numbers. Development of a reliable method for nonzero Froude-number calculations of a ship's boundary layer should have high priority . 
Adachi, H. \& Hinatsu, M. (1981), "On Methods of Separation of Resistance Components of Thin Ships", J. Kansai Soc. N.A., Japan, No. 183.

Beck, R.F. (1971), "The Wave Resistance of a Thin Ship with a Rotational Wake", J. Ship Research, Vol. 15, No. 3.

Betz, A. (1925), "A Method for the Direct Determination of Wing Section Drag", NACA - T.M. 337.

Brard, R. (1970), "Viscosity, Wake and Ship Waves", J. Ship Research, Vol. 14, No. 4 .

Bryer, D.W., Walshe, D.E. \& Garner, H.C. (1955), "Pressure Probes Selected for Mean Flow Measurements. Exploration of Turbulent Boundary Layers", Rep. Memor. Aero. Res. Conn., London, No. 3037.

Cebeci, T. \& Smith, A.M.O. (1974), "Analysis of Turbulent Boundary Layers", Academic Press, New York.

Chang, K.C. \& Patel, V.C. (1975), "Calculation of Three-Dimensional Boundary Layers on Ship Forms", The University of Iowa, Iowa Inst. Hydraulic Res. Report No. 178.

Cramer, J. (1984), "The IIHR Scanivalve Computer Positioning Ciruit", Iowa Inst. Hydraulic Res. Tech. Rep. No. 276.

Emerson, A. (1954), "The Application of Wave-Resistance Calculations to Ship Hul1 Design", T.I.N.A., 96, p. 268.

Emerson, A. (1967), "The Calculation of Ship Resistance: An Application of Guilloton's Method", Tran. R.I.N.A., Vol. 109, No. 3.

Gadd, G.E. (1963), "A Brief Note on Boundary Layer Effects on Ship Waves", N.P.L. Ship Division T.M. 23.

Gadd, G.E. (1973), "Wave Resistance Calculation by Guilloton's Method", Tran. R.I.N.A. Vol. 115.

Gadd, G.E. (1976), "Wave Theory Applied to Practical Hull Forms", Proc. International Seminar on Wave Resistance, Japan.

Goldstein, S. (1936), "A Note on Measurement of Total Head and Static Pressure in a Turbulent Stream", Proc. Royal Soc. Al55.

Guilloton, R. (1948), "Streamlines on Fine Hulls", Trans. of R.I.N.A. London.

Guilloton, R. (1951), "Potential Theory of Wave Resistance of Ships with Tables for its Calculation", Trans. SNAME, Vol. 59, p. 86.

Ha11, I.M. (1956), "The Displacement Effect of a Sphere in a Two-Dimensional Shear Flow", J. of Fluid Mech., Vol. 2. 
Havelock, T.H. (1935)", Ship Waves: The Relative Efficiency of Bow and Stern", Proc. Inst. Eng. \& Shipb. Scotland, 81, p. 187.

Havelock, T.H. (1948), "Calculation Illustrating the Effect of Boundary Layer on Wave Resistance", Trans. Inst. of Naval Architects, Vol. 90.

Inui, T. (1957), "Study on Wavemaking Resistance of Ships", 60th Anniversary Series, Soc. of Naval. Arch. of Japan, Vol. 1.

Ju, S. (1983), "Study of Total and Viscous Resistance for the Wigley Parabolic Ship Form", IIHR Rep. No. 261.

Kang, S.H. (1978), "Viscous Effects on the Wave Resistance of a Thin Ship", The University of Iowa, Ph.D. Thesis.

Landweber, L. (1971), "Characteristics of Ship Boundary Layers", Proc. 8th Symp. of Naval Hydrodynamics, Pasadena.

Landweber, L. (1977), "Effect of Wake on Wave Resistance", Proc. of 18th American Towing Tank Conference, Annapolis.

Landweber, L. (1978), "Irrotational FLow Equivalent to Boundary Layer and Wake", The 5th David W. Taylor Lectures, DTNSRDC - 78/111.

Landweber, L. (1979), "Wigley Parabolic Hull Group Discussion", Proc. of the Workshop on Ship Wave-Resistance Computations, DWTNSRDC, Bethesda, Maryland.

Landweber, L. (1981), "Irrotational Flow Within the Boundary Layer and Wake", The 4th G. Weinblum Memorial Lecture, University of Hamburg, J. of Ship Research, V.26, No. 4, Dec. 1982, p. 219-228.

Landweber, L. \& Celik, I. (1980), "Wave Resistance of Wigley Parabolic Ship Form from Second-Order Double-Model Source Distribution", Workshop on Ship Wave-Resistance Comput., Japan.

Landweber, L. \& Ju, S. (1983), "A Slender-Body Solution for the Centerplane Distribution for the Wigley Ship Form", DWTNSRDC Workshop, Bethesda, Maryland.

Landweber, L. \& Nakayama, A. (1975), "Effect of Tank Wall on Ship-Model Resistance", Report of the Resistance Committee, Appendix 6, 14th International Towing Tank Conference.

Landweber, L. \& Patel, V.C. (1979), "Ship Boundary Layers", Annual Review of Fluid Mechanics, Vol. II.

Landweber, L. \& Wu, J. (1963), "The Determination of Viscous Drag of Submerged and Floating Bodies by Wake Survey", J. Ship Research, Vol. 7, No. 1.

Lavrentiev, V.W. (1951), "The Influence of the Boundary Layer on the Wave Resistance of a Ship", Akad. Nauk. USSR 80-6.

Lighthill, J. (1958), "On Displacement Thickness", J. of Fluid Mech. Vol. 4. 
Lurye, J.R. (1968), "Interaction of Free Surface Waves with Viscous Wakes", The Physics of Fluids, Vol. 10, No. 2.

MacMillen, F.A. (1956), "Experiments on Pitot-Tubes in Shear Flows", A.R.C. Rep. Memo. 3028.

Martin, M. (1958), "The Iowa Towing Tank", IIHR Rep. Nov. 1958.

Maruo, H. (1976), "Ship Waves and Wave Resistance in a Viscous Fluid", Int. Seminar on Wave Resistance, Japan.

Merriam, K.G. \& Spaulding, E.R. (1935), "Comparative Tests of Pitot-Static Tubes", NACA TN 546.

Michell, J.H. (1898), "The Wave Resistance of a Ship", Phil. Mag. (5), 45, p. 106.

Milgram, J.H. (1969), "The Effects of a Wake on the Wave Resistance of a Ship", J. of Ship Research, Vol. 13, No. 1.

Miloh, T. \& Landweber, L. (1980), "Ship Centerplane Source Distributions", J. of Ship Research, Vol. 24, No. 1.

Miloh, T. \& Patel, V.C. (1973), "Orthogonal Coordinate Systems for ThreeDimensional Boundary Layers with Particular Reference to Ship Forms", J. of Ship Research, Vol. 17, No. 4.

Moreno, M., Perez-Rojas, L. \& Landweber, L. (1975), "Effect of Wake on Wave Resistance of a Ship Model", IIHR Rep. No. 180.

Mori, K. (1978), "Viscous Effects on Wave Resistance", Part D of "Study on Local Non-Linear Effects in Ship Waves", Int. Joint Research, Japan Soc. for Promotion of Science.

Nagamatsu, T. (1979), "Comparison Between Calculated and Measured Results of Turbulent Boundary Layers Around Ship Models", Mitsubishi Tech. Bull. No. 133.

Nash, J. \& Patel, V.C. (1972), "Three-Dimensional Turbulent Boundary Layers", SBC Technical Books, Atlanta.

Newman, J.N. (1976), "Linearized Wave Resistance Theory", Proc. International Seminar on Wave Resistance, Japan.

Okabe, J. \& Jinnaka, T. (1950), "On the Waves of the Ships", Rep. of Res. Inst. for Fluid Engineering, Kyushu University, 7-1.

Preston, J.H. (1945), "The Effect of the Boundary Layer and Wake on the Flow Past a Symmetrical Airfoil at Zero-Incidence; Part I, The Velocity Distribution at the Edge of, and Outside the Boundary Layer and Wake", Aero Res. Council, R\&M No. 2107. 
Patel, V.C., Sarda, O.P. \& Shahshahan, A. (1983), "Calculation of Ship Boundary Layers", Proc. 4th Symp. Turb. Shear Flow, Karlsruhe, FRG.

Rajaratnam, N. \& Muralindhar, D. (1968), "Yaw Probe Used as a Preston Tube", Aero J. of Royal Aero. Soci. Vol. 72.

Ramaprian, B.R., Patel, V.C. \& Choi, D.H. (1978), "Mean Flow Measurements in the Three-Dimensional Boundary Layer Over a Body of Revolution at Incidence", IIHR L.D. Rep. No. 56.

Sarda, O.P. (1985), "Turbulent Flow Past Ship Hulls--an Experimental and Computational Study", The Univ. of Iowa, Ph.D. Thesis.

Schoenherr, K.E. (1932), "Resistance of Flat Surfaces Moving through a Fluid", Trans. SNAME, Vol. 40.

Shahshahan, A. (1981), "Study of Free-Surface Flow Around a Ship's Bow", The University of Iowa, M.S. Thesis.

Shahshahan, A. \& Patel, V.C. (1983), "The Boundary Layer at Hull-Wave Intersections", Proc. 18th Midwestern Conf. on Mech., Iowa City.

Shaw, R. (1960), "The Influence of Hole Dimension on Static Pressure Measurements", J. of Fluid Mechanics, Vol. 7.

Shearer, J.R. \& Cross, J.J. (1965), "The Experimental Determination of the Components of Ship Resistance for a Mathematical Model", The Royal Inst. of Naval Arch.

Swain, A. \& Landweber, L. (1974), "Experimental Study of the Wavemaking of Horizontally-Oriented Vorticity in a Wake", IIHR Rep. No. 153.

Tatinclaux, J.C. (1970), "Effects of a Rotational Wake on the Wave Resistance of an Ogive", J. Ship Research, Vol. 14, No. 2.

Tatinclaux, J.C. (1970), "Experimental Investigations of the Drag Induced by Bilge Vortices", Schiffstechnik, Heft 87.

Tzou, T.S. (1966), "Secondary Flow Near a Simulated Free Surface", The Univ. of Iowa, M.S. Thesis.

Walsche, D.E. \& Garner, H.C. (1960), "Usefulness of Various Pressure Probes in Fluctuating Low-Speed Flows", Brit. ARC 21714.

Webster, W.C. \& Huang, T.T. (1970), "Study of the Boundary Layer on Ship Forms", J. Ship Research, Vol. 14.

Weinblum, G. (1930), "Anwendungen der Michellschen Widerstands-Theorie", J.S.T.G. 1930, P. 389.

Weinblum, G., Kendrick, J. \& Todd, M.A. (1952), "Investigation of Wave Effects Produced by a Thin Body--TMB Model 4215", DWTNSRDC Report 840 . 
Wigley, W.C.S. (1937), "Effect of Viscosity on the Wavemaking of Ships", Trans. Inst. Eng. \& Shipb. Scotland, 81, p. 187.

Wigley, W.C.S. (1942), "Calculated and Measured Wave Resistance of Series of Forms", Trans. I.N.A. p. 70 .

Wigley, W.C.S. (1963), "Effects of Viscosity on Wave Resistance", Int. Seminar on Theoretical Wave Resistance, Ann Arbor.

Wilson, M.B. (1971), "A Michell Oseen-Flow Theory for Thin Ships", California Inst. of Tech., Ph.D. Thesis.

Wu, T.Y. (1963), "Interaction Between Ship Waves and Boundary Layer", Int. Seminar on Theoretical Wave Resistance, Ann Arbor. 

